Florida International University FIU Digital Commons

9-18-2017

\title{
Trapped Ion Mobility Spectrometry coupled to Fourier Transform Ion Cyclotron Resonance Mass Spectrometry for the analysis of Complex Mixtures.
}

Paolo Benigni

Florida International University, pbeni001@fiu.edu

DOI: $10.25148 /$ etd.FIDC004019

Follow this and additional works at: https://digitalcommons.fiu.edu/etd

Part of the Analytical Chemistry Commons, and the Environmental Chemistry Commons

\section{Recommended Citation}

Benigni, Paolo, "Trapped Ion Mobility Spectrometry coupled to Fourier Transform Ion Cyclotron Resonance Mass Spectrometry for the analysis of Complex Mixtures." (2017). FIU Electronic Theses and Dissertations. 3547.

https://digitalcommons.fiu.edu/etd/3547 


\section{FLORIDA INTERNATIONAL UNIVERSITY}

Miami, Florida

TRAPPED ION MOBILITY SPECTROMETRY COUPLED TO FOURIER

TRANSFORM ION CYCLOTRON RESONANCE MASS SPECTROMETRY FOR THE ANALYSIS OF COMPLEX MIXTURES.

A dissertation submitted in partial fulfillment of

the requirements for the degree of

DOCTOR OF PHILOSOPHY

in

CHEMISTRY

by

Paolo Benigni 
To: Dean Michael R. Heithaus

College of Arts, Sciences and Education

This dissertation, written by Paolo Benigni, and entitled Trapped Ion Mobility Spectrometry coupled to Fourier Transform Ion Cyclotron Resonance Mass Spectrometry for the Analysis of Complex Mixtures, having been approved in respect to style and intellectual content, is referred to you for judgment.

We have read this dissertation and recommend that it be approved.

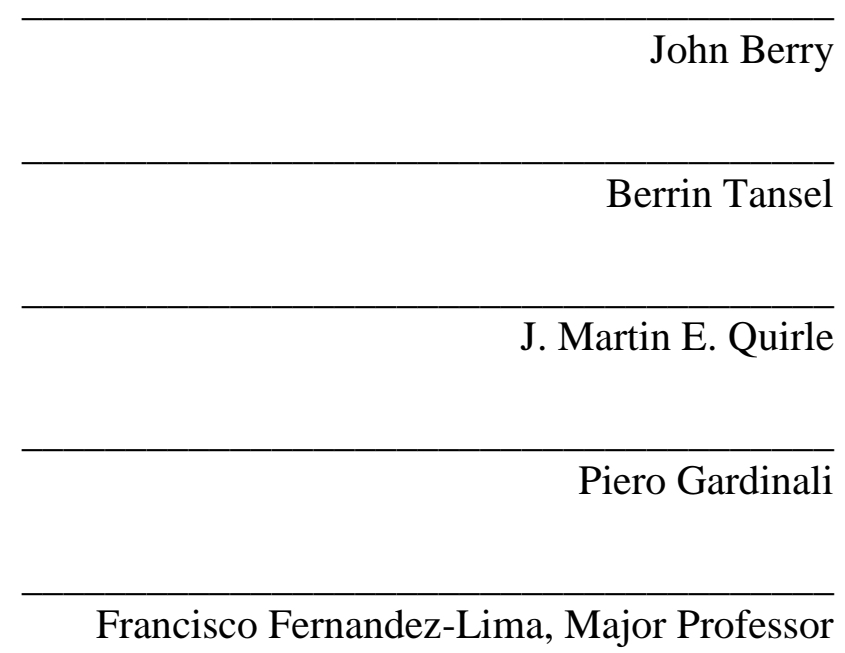

Date of Defense: September 18, 2017

The dissertation of Paolo Benigni is approved.

Dean Michael R. Heithaus

College of Arts, Sciences and Education

Andrés G. Gil

Vice President for Research and Economic Development and Dean of the University Graduate School

Florida International University, 2017 
(C) Copyright 2017 by Paolo Benigni

All rights reserved. 


\section{DEDICATION}

I dedicate this dissertation to the love of my life and my wife Miriam Kay, my parents, Stefano and Lucia, and all my siblings Luigi, Bernardo and Katrina, Maria, Tommaso, Filippo, Judith, Anna, Claire, Jonathan. I would also like to thank Anthony Reid and all the teachers and professors that have believed in me throughout all my school years. 


\section{ACKNOWLEDGMENTS}

I would like to thank my advisor, Francisco Fernandez-Lima, without whom none of this work would have ever happened. For taking me into your research group and helping me grow as a scientist, always pushing me to be the best that I could be. For your continuous support, and allowing me to travel to as many conferences as I could get my hands on. Kendra, Alyssa, Alan, Camilo, Anthony and all the past and present members of the Fernandez-Lima lab and the Advanced Mass Spectrometry Facility, for your patience. I would like to thank Bruker Daltonics for their collaboration and continual support over these many years, and in particular Mark E. Ridgeway, Christopher J. Thompson, and Melvin A. Park, without whom much of this work would not have been possible.

I would like to thank my committee members, Piero Gardinali, Berrin Tansel, J. Martin E. Quirke, and John Berry for their support, advice, and whose guidance has helped me grow and improve the quality of my research. I would also like to acknowledge Todd Crowl and Rita Teutonico, Bradley Schonhoff, Mark Kershaw, and Scott Graham, and the rest of the CREST CAChE center at FIU and the fellowship that I received through the center. I would like to acknowledge the School of Integrated Science and Humanity, and the Department of Chemistry and Biochemistry for the support that I have received, and for all the faculty and staff that have helped me though out my years here. In particular I would like to thank Jackelyn Marcos, Maggie Autie, Alexander Mebel, Watson Lees, Stephen Winkle, Jaroslava Mikšovská, and all of the other faculty that have helped me learn and grow as a scientist while at FIU. I would like to acknowledge the Instructional and Research Computing Center and the staff at the High-Performance Computational resources that enabled me to further my research. 


\title{
ABSTRACT OF THE DISSERTATION \\ TRAPPED ION MOBILITY SPECTROMETRY COUPLED TO FOURIER \\ TRANSFORM ION CYCLOTRON RESONANCE MASS SPECTROMETRY FOR \\ THE ANALYSIS OF COMPLEX MIXTURES
}

\author{
by
}

\section{Paolo Benigni}

Florida International University, 2017

Miami, Florida

\section{Professor Francisco Fernandez-Lima, Major Professor}

\begin{abstract}
Analytical Characterization of complex mixtures, such as crude oil, environmental samples, and biological mixtures, is challenging because of the large diversity of molecular components. Mass spectrometry based techniques are among the most powerful tools for the separation of molecules based on their molecular composition, and the coupling of ion mobility spectrometry has enabled the separation and structural elucidation using the tridimensional structure of the molecule. The present work expands the ability of analytical chemists by furthering the development of IMS-MS instrumentation by coupling Trapped Ion Mobility Spectrometry to Fourier Transform Ion Cyclotron Resonance Mass Spectrometry (TIMS-FT-ICR MS). The TIMS-FT-ICR MS platform combines the highresolution separation of TIMS, which has mobility resolving powers up to 400, and ultrahigh mass resolution of FT-ICR MS, with mass resolving power over 1,000,000. This instrumentation allows the assignment of exact chemical composition for compounds in a
\end{abstract}


complex mixture, as well as measurement of the collision cross-section of the molecule. Herein, the principles of the TIMS separation and its coupling to FT-ICR MS are described, as well as how the platform can be applied to targeted analysis of molecules, and untargeted characterization of complex mixtures.

Molecular standards were analyzed by TIMS-MS in order to develop a computational workflow that can be utilized to elucidate molecular structure, using the measured collision cross-section of the ion. This workflow enabled identification of structural, cis/trans isomers, and chelated molecules and provides the basis for unsupervised structural elucidation of a complex mixture, and in particular for the elucidation of hydrocarbons from fossil fuels. In summary, this work presents the coupling of TIMS-FT-ICR MS and provides examples of applications as a proof of concept of the potential of this platform for solving complex analytical challenges. 


\section{TABLE OF CONTENTS}

CHAPTER $\quad$ PAGE

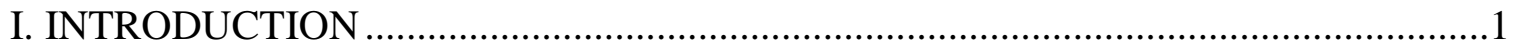

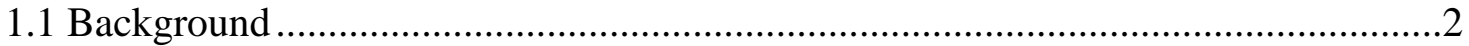

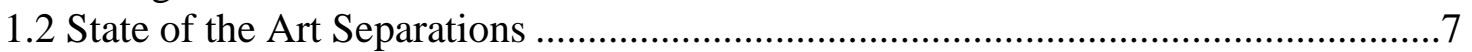

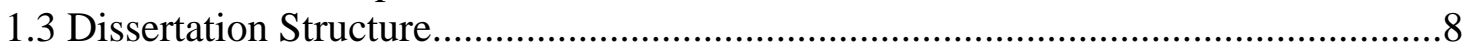

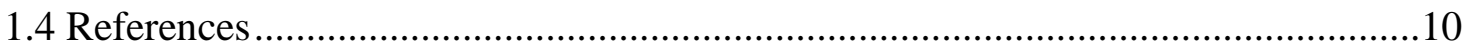

II. INCREASING POLYAROMATIC HYDROCARBON (PAH) MOLECULAR COVERAGE DURING FOSSIL OIL ANALYSIS BY COMBINING GAS CHROMATOGRAPHY AND ATMOSPHERIC-PRESSURE LASER IONIZATION FOURIER TRANSFORM ION CYCLOTRON RESONANCE MASS

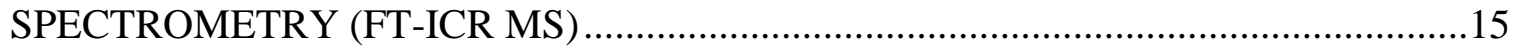

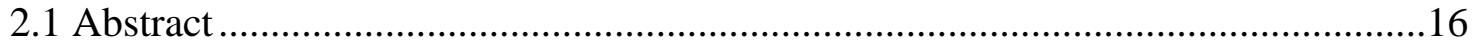

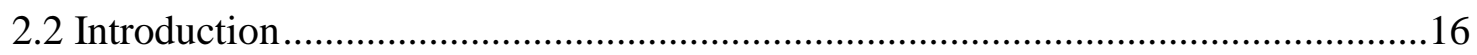

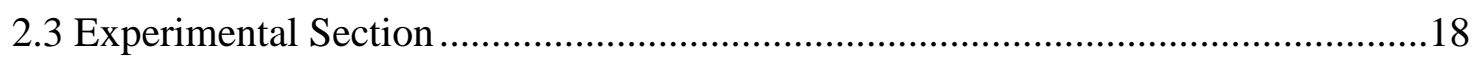

2.3.1 Sample Preparation ...............................................................................18

2.3.2 APLI-FT-ICR MS Analysis ......................................................................19

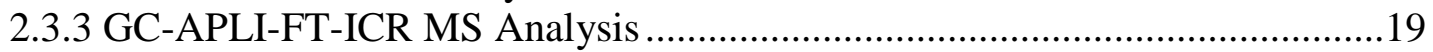

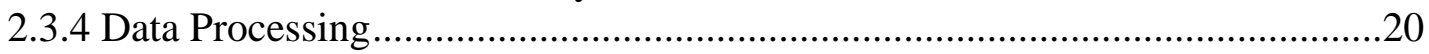

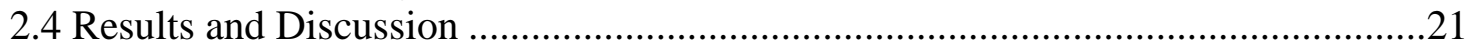

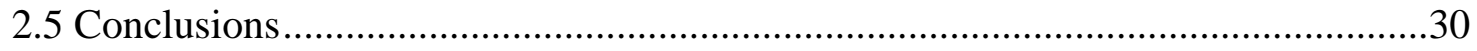

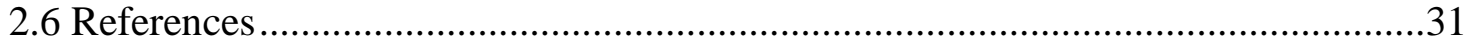

III. FAST SCREENING OF POLYCYCLIC AROMATIC HYDROCARBONS USING TRAPPED ION MOBILITY SPECTROMETRY - MASS SPECTROMETRY 36

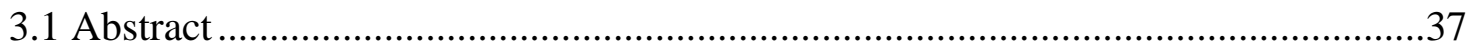

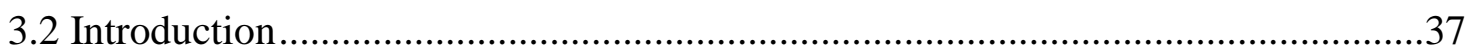

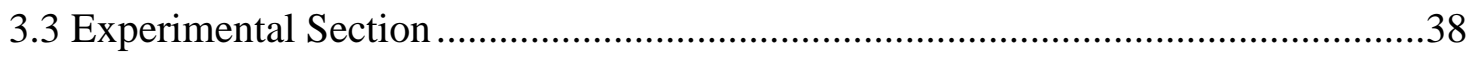

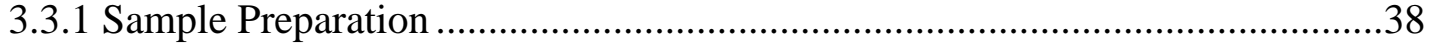

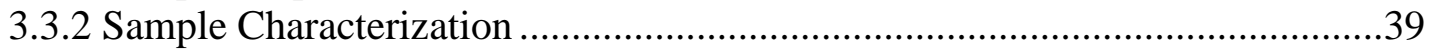

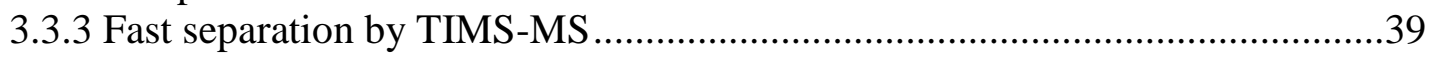

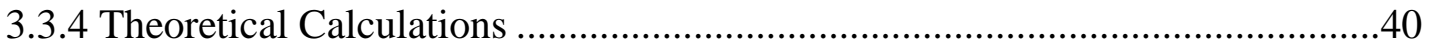

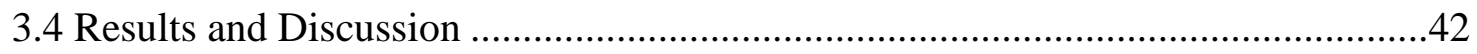

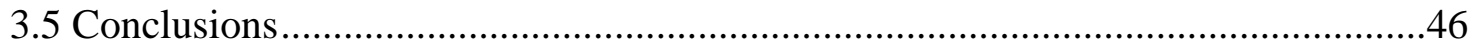

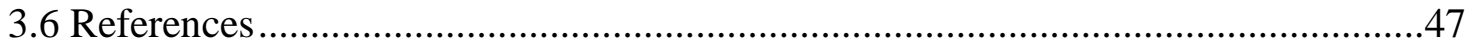

IV. ANALYSIS OF GEOLOGICALLY RELEVANT METAL PORPHYRINS USING TRAPPED ION MOBILITY SPECTROMETRY-MASS SPECTROMETRY

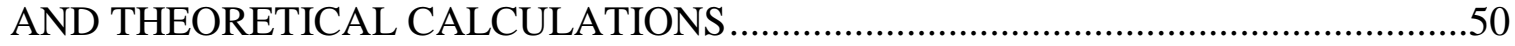

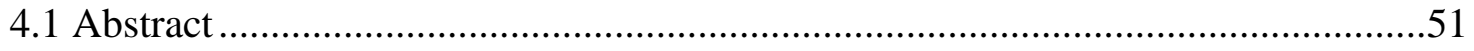




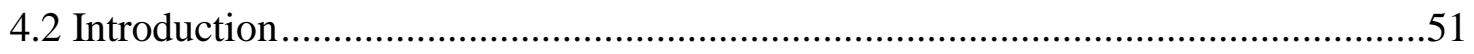

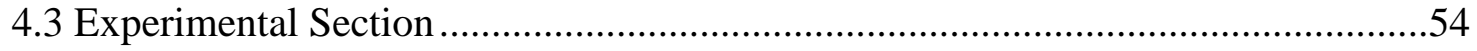

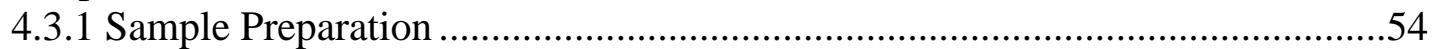

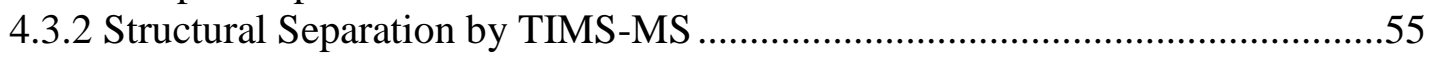

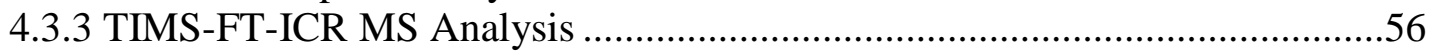

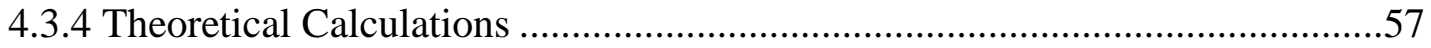

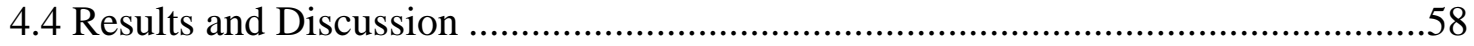

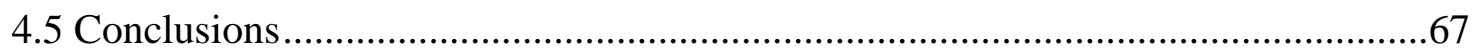

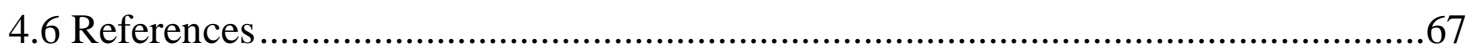

V. TOWARDS UNSUPERVISED POLYAROMATIC HYDROCARBONS STRUCTURAL ASSIGNMENT FROM SA-TIMS -FTMS DATA.................................73

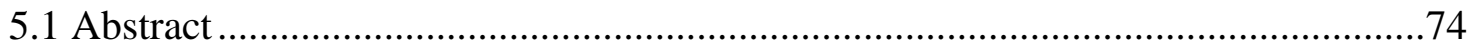

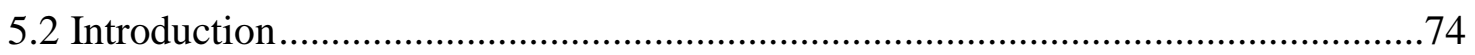

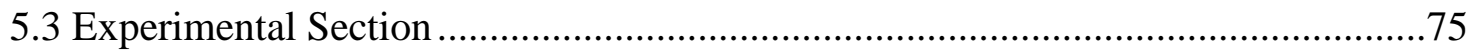

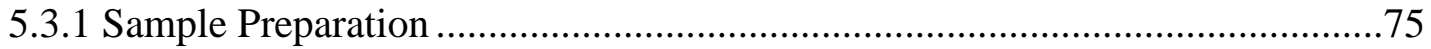

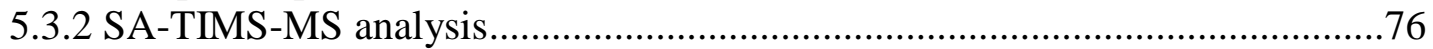

5.3.3 Theoretical methods ....................................................................................

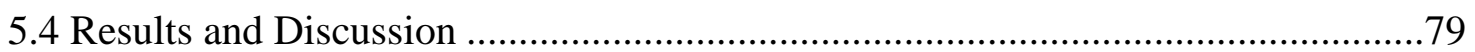

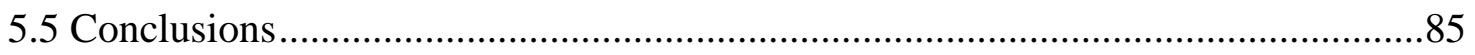

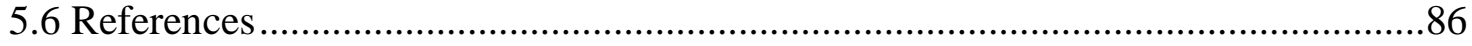

VI TARGETED HIGH-RESOLUTION ION MOBILITY SEPARATION COUPLED TO ULTRAHIGH-RESOLUTION MASS SPECTROMETRY OF ENDOCRINE

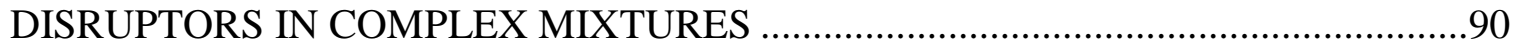

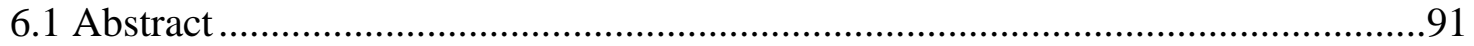

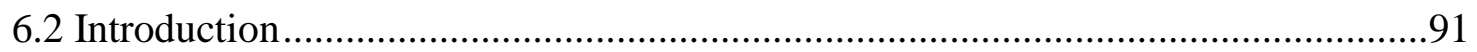

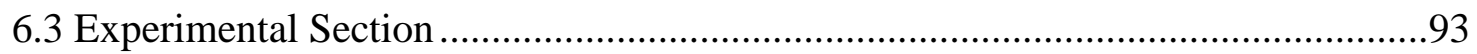

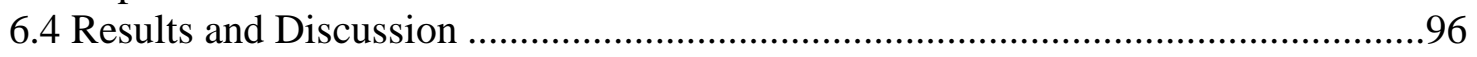

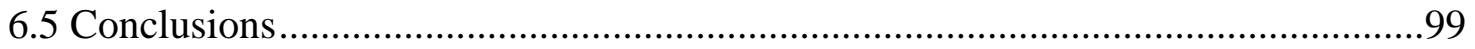

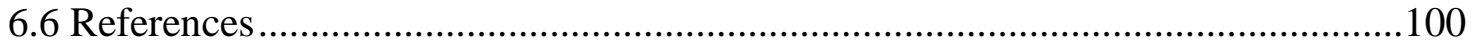

VII. OVERSAMPLING SELECTIVE ACCUMULATION TRAPPED ION MOBILITY SPECTROMETRY COUPLED TO FT-ICR MS: FUNDAMENTALS

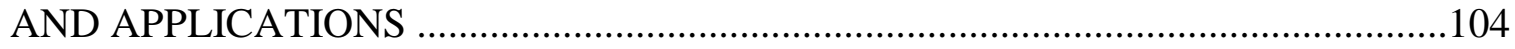

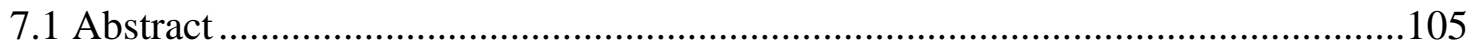

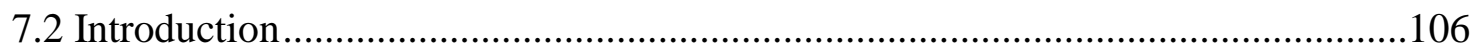

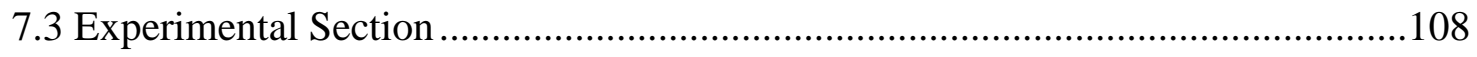

7.3.1 Materials and Reagents .......................................................................108

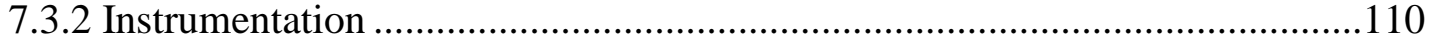

7.3.3 SA-TIMS versus OSA-TIMS Operation ..................................................111

7.3.4 Mobility and Collision Cross Section Calculations .........................................112

7.3.5 Experimental Parameters ............................................................................113

7.3.6 OSA-TIMS-FT-ICR MS Data Processing ……….....................................115

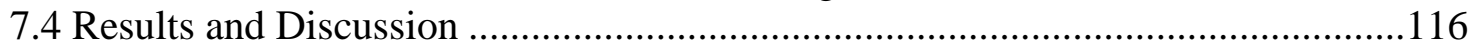




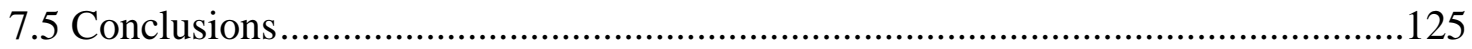

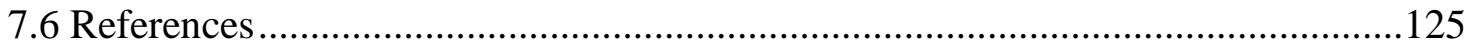

VIII. ANALYSIS OF PHOTOIRRADIATED WATER ACCOMMODATED

FRACTIONS OF CRUDE OILS USING TANDEM TIMS AND FT-ICR MS .............132

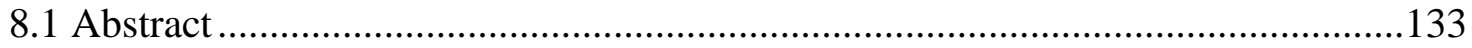

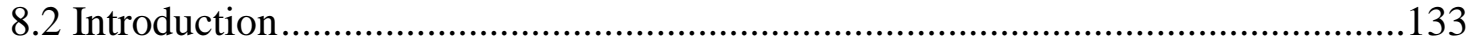

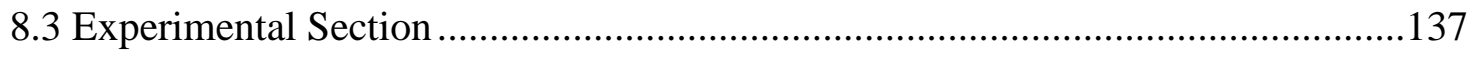

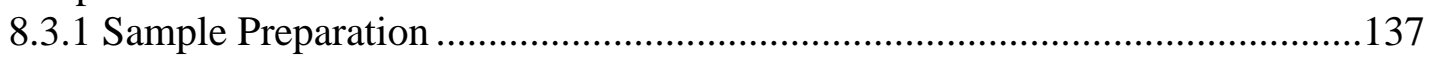

8.3.2 WAF exposure to Light and Extraction ...................................................138

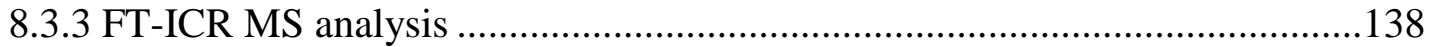

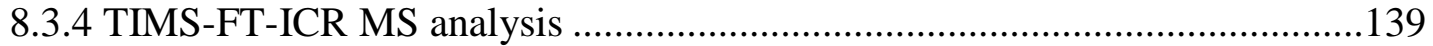

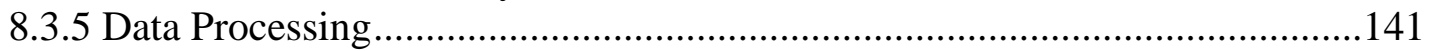

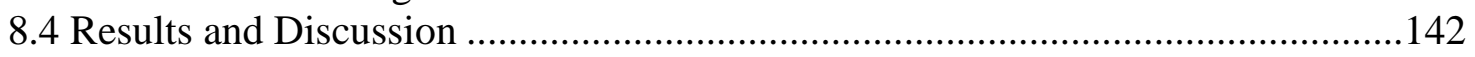

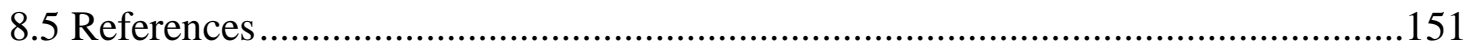

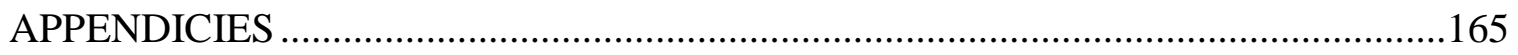

VITAE 


\section{LIST OF TABLES}

TABLE

PAGE

2.1. Reported Compounds Observed by APLI-FT-ICR MS and GC-FT-ICR MS in the Organics in Shale Crude Oil and Petroleum Crude Oil Certificates.

4.1. Experimental Mass $(\mathrm{m} / \mathrm{z})$, Mobility (Ko), and CCS for Me-OEP Studied Using TIMS-FT-ICR MS.

4.2. Results from the Theoretical Calculations, Illustrating the Changes in Bond Length between the Nitrogen Atoms in Pyrrole (See Figure 4.3 for Labels), the Bridge Carbons, the Dihedral Angle between Nitrogen, and the Angle between the Bridge.

6.1. Experimental SA-TIMS-FTMS and Theoretical Mass and Mobility Values for Bisphenol A, Diethylstilbestrol, Estrone, Hexestrol, $\alpha$-Estradiol, and 17- $\alpha$ -

Ethynylestradiol.

7.1. List of Compounds Assigned Based on the Accurate Mass and Mobility from the 2D-IMS-MS Contour Plot Obtained Using APPI-OSA-TIMS-FT-ICR MS from a Complex Mixture of Coal Tar.

8.1. Table of Identified Ion Formulas for $m / z 327$ and 489 as found in Figure 8.2. .145 


\section{LIST OF FIGURES}

FIGURE

PAGE

2.1. Typical FT-ICR MS spectra of (a) petroleum crude oil (PCO), (b) organics in shale oil (OSO), and (c) heavy sweet crude oil (HSO) obtained by direct-infusion APLI-FTICR MS (top, black spectrum) and GC-APLI-FT-ICR MS (bottom, red spectrum). Also shown is the time domain signal for the direct-infusion analysis (black) and a single scan in the GC-FTMS analysis, along with the total ion chromatogram (red).

2.2. Double-bond equivalent (DBE) versus carbon number for the $\mathrm{HC}, \mathrm{N}, \mathrm{O}$ and $\mathrm{S}$ classes of (a) petroleum crude oil, (b) organics in shale oil (OSO), and (c) heavy sweet oil (HSO) obtained by direct-infusion APLI-FT-ICR MS (top) and GC-APLI-FT-ICR MS (bottom)

2.3. Percentages of compounds observed only by GC (GC only, red), common between both analysis (blue), and seen by infusion only (black) in the $m / z$ 100-450 range, compared to the total number of identifications, as a function of the heteroatom class in (a) petroleum crude oil (PCO), (b) organics in shale oil (OSO), and (c) heavy sweet oil (HSO)

2.4. 2D GC-FT-ICR MS contour plots, mass spectrum projections, and selected GC traces for (a) $m / z, 178$ and (b) $m / z, 256$ for the organics in shale oil (OSO). Peak assignments can be found in Appendix 2.6.

2.5. Two-dimensional (2D) GC-FT-ICR MS contour plots including the double-bond equivalent (DBE, color scale) and relative abundance (symbol size) for the primary heteroatom PAH classes in (a) petroleum crude oil (PCO), (b) organics in shale oil (OSO), and (c) heavy sweet oil (HSO).

2.6. Typical mass resolution as a function of the transient time in a FT-ICR MS with an infinity cell (7.0 T magnet) using the magnitude and absorption modes. Notice that high mass resolution can be achieved for short GC peaks (5s) and ultrahigh mass resolution can be achieved for GC peaks in the range of 12-20 s, assuming 15 FTICRMS acquisitions per GC peak.

3.1. Typical TIMS spectra (Tramp $=500 \mathrm{~ms})$ of (a) pyrene $(\mathrm{R}=90)$, (b) chrysene $(\mathrm{R}=$ 103), (c) triphenylene $(\mathrm{R}=107)$, (d) 1,2-benzanthracene $(\mathrm{R}=98)$, (e) 2, 3benzanthracene $(\mathrm{R}=96)$, (f) perylene $(\mathrm{R}=113)$, $(\mathrm{g})$ benzo(a)pyrene $(\mathrm{R}=133)$, (h) benzo(e)pyrene $(R=102)$, (i) pentacene $(R=96)$, (j) 1,2,3,4-dibenzanthracene $(R=$ $155),(\mathrm{k}) 1,2: 5,6$-dibenzanthracene $(\mathrm{R}=98)$ and $(\mathrm{l})$ rubrene $(\mathrm{R}=125)$. In the inset, the DFT/B3LYP/6-31++g(d,p) isosurface and a space-filled model representation of the molecular ions is shown. 
3.2. TIMS spectra as a function of the ramp time (100-800 ms) for two component isomeric mixtures of triphenylene (a), chrysene (b), 1,2-benzanthracene (c), and 2,3benzanthracene (d). The TIMS peak resolution is shown for single components.

3.3. 2D TIMS-MS contour plot of a fast scan (low resolution $\mathrm{R}=30-60$ ) of a PAH mixture in soil (SC017) featuring over 16 different PAHs, including multiple sets of structural isomers. Regions a, b, and c correspond to PAH isomers with chemical formula $\mathrm{C} 14 \mathrm{H} 10, \mathrm{C} 18 \mathrm{H} 12$, and $\mathrm{C} 2 \mathrm{OH} 12$, respectively.

3.4. TIMS and MS projection plots for the insets shown in Fig. 3.3 of the structural isomers (a) phenanthracene and anthracene (not resolved), (b) chrysene (56\%) and 1,2benzanthracene (44\%), and (c) benzo(a)pyrene (45\%), benzo(b)fluoranthene(14\%) and benzo(k)fluoranthene (41\%). (d-f) Excerpts of the mass spectrum corresponding to the compounds in $(\mathrm{a}-\mathrm{c})$.

4.1. Mobility spectra of Me-OEP obtained using APPI-TIMS-FT-ICR MS. Note the single- and dual-band mobility profiles observed for $\mathrm{Mn}, \mathrm{Zn}$, and Ni compared to the vanadyl $(\mathrm{V}=\mathrm{O})$ and titanyl $(\mathrm{Ti}=\mathrm{O})$, respectively.

4.2. Distribution of four commonly encountered molecular classes (HC, N, O, and S) observed in the coal tar APPI-TIMS-FTICR MS analysis and the OEP unique space based on their $\mathrm{m} / \mathrm{z}$ and inverse mobility $1 / \mathrm{K} 0$. Note the area occupied by OEPs is shifted to a greater $\mathrm{m} / \mathrm{z}$ than other molecules within the aromatic region of the $2 \mathrm{D}$ IMS-MS plot.

4.3. Projections of the three-dimensional (3D)-optimized structures for the different OEPs. The radical OEP, not observed experimentally, is labeled with the bridge carbons (green circles) as well as the notation for the distances and angles shown in Table 4.2. Protonation sites are highlighted in red circles. Note that the deviations from planarity depend upon the protonation site. More details can be found in Table 4.2.

4.4 Energy landscape diagram showing the change in energy for each protonation site calculated for the vanadyl and titanyl structures. Note that the theoretical CCS are fairly similar for all of the structures, even though each structure is unique and the energies are different.

5.1. Typical IMS-MS contour plot generated by APLI-SA-TIMS-FTMS for the Coal Tar SRM and b) Mobility as a function of the $\mathrm{m} / z$ (DBE number in colorscale) for the CxHy class.

5.2. Number of structures at nominal CCS for a) $\mathrm{C}_{10} \mathrm{H}_{8}$ and b) $\mathrm{C}_{12} \mathrm{H}_{8}$. Structures within $2 \%$ of the experimental value (*) are shown for $\mathrm{C}_{10} \mathrm{H}_{8}$ and $\mathrm{C}_{12} \mathrm{H}_{8}$ in c) and d), respectively. 
5.3. a) Number of structures as a function of the carbon number for $\mathrm{C}_{\mathrm{x}} \mathrm{H}_{2 \mathrm{x}-4} . \mathrm{b}$ ) Number of structures as a function of the DBE number, and c) estimated computational time as a function of the DBE.

5.4. Number of potential structures dependence on the "chemical constraints". .84

6.1. (a) 2D-IMS-FTMS contour plot of the complex mixture containing EDC compounds (dashed square) and SRFA standard. (b) Number of peaks identified at the nominal mass in SA-TIMS-FTMS and FTMS analysis.

6.2. (a) 2D-IMS-FTMS contour plot of the complex organic mixture containing EDC compounds and SRFA standard. Notice the separation of (1) bisphenol A, (2) diethylstilbestrol, (3) estrone, (4) hexestrol, (5) $\alpha$-estradiol, (6) 17- $\alpha$-ethynylestradiol from the SRFA matrix. 2D-IMS-FTMS contour plot at the nominal mass for the structural isomers of (E)- and (Z)-diethylstilbestrol (left) and estrone and hexestrol (right) in (b) the complex sample, (c) the reference standards, and (d) the respective FTMS projections (* denotes the EDC formula).

7.1. (a) Schematics of the TIMS cell and (b) profile of the voltage (black) and electric field (blue) across a simplified representation of the electrodes in the TIMS analyzer. .109

7.2. Comparison of the analysis sequence, electric field, and voltage applied in the TIMS analyzer during SA-TI.MS (top) and OSA-TIMS (bottom) for the analysis of the same mobility range using 5 elution steps and 20 elution steps. Notice that in SATIMS $\Delta$ Vramp $=$ Vstep and in OSA-TIMS $\Delta$ Vramp $>$ Vstep.

7.3. Theoretical IMS profiles utilizing a single and a double simulated peak with a fwhm of $1 \mathrm{~V}$ using selective accumulation SA (left) and oversampling OSA (right). Notice the number of points across the IMS peaks based on the trapping voltage $\left(\mathrm{V}_{\text {ramp }}\right)$ and the voltage steps $\left(\mathrm{V}_{\text {step }}\right)$ : SA-TIMS $\mathrm{V}_{\text {ramp }}=\mathrm{V}_{\text {step }}$ and OSA-TIMS $\mathrm{V}_{\text {ramp }} \gg$ $\mathrm{V}_{\text {step. }}$

7.4. Typical experimental IMS profiles using selective accumulation SA (left) and oversampling OSA (right) TIMS-FT-ICR MS for the TuningMix standards at $\mathrm{m} / \mathrm{z}$ (a) 922, (b) 1222, and (c) 1522. Notice the differences in resolving power and signal-tonoise ratio between SA-TIMS and OSA-TIMS.

7.5. Typical IMS profiles using SA-TIMS (left) and OSA-TIMS (right) FT-ICR MS for (a) structural (SDGRG and GRGDS) and (b) conformational (ATHP 3) peptide isomers. Notice the high mass accuracy of the FT-ICR MS measurements.

7.6. (a) Typical 2D-IMS-MS contour plot obtained using APPI-OSA-TIMS-FT-ICR MS from a complex mixture of coal tar. Two regions of interest are clearly discernible, resulting in a more aliphatic region (blue) and a more aromatic region (gold). (b) A closer view of $m / z 282-284$ is shown, as well as the unsupervised fits for (c) a single and (d) multiple mobility band distributions. 
8.1. Typical FT-ICR MS spectrum and 2D TIMS-FT-ICR MS contour plot for the WAF (a) at 0 and (b) $115 \mathrm{~h}$ light exposed. The number of peaks identified per unit $\mathrm{m} / \mathrm{z}$ in the MS domain (black) and TIMS-MS (blue) domains are also shown for (a) and (b). Notice the significant differences in the number of identifications between (a) and (b), as well as between the MS and TIMS MS experiments, increasing the level of molecular features identified per analysis.

8.2. Typical 2D TIMS-FT-ICR MS contour plot and MS projections for $m / z$ a) 327 and b) 489. IMS projections for specific chemical formulas (connected scatterplot) with the unsupervised fitting by the SAME package of minimum number of mobility features is shown. Note that the SAME package relies on the experimental profile of the distribution, which is able to show multiple features due to the high resolving power of the TIMS analyzer. Formula assignments are provided in Table 8.1.

8.3. Typical size dependence (CCS) with carbon number for the $\mathrm{O}_{0-4}$ chemical classes observed in the WAF samples as a function to exposure to light (t-0h, $-16 \mathrm{~h}$, and $-115 \mathrm{~h}$ ). The color scale corresponds to the number of rings and double bond equivalents (DBE) of a molecule. Note the large increase in assignments between $\mathrm{t}-\mathrm{Oh}$ and $\mathrm{t}-16 \mathrm{~h}$, as well as increases in oxygen content between $\mathrm{t}-16 \mathrm{~h}$ and $\mathrm{t}-115 \mathrm{~h}$.

8.4. Total number of molecular feature assignments based on chemical formula alone from FT-ICR MS measurements and based on chemical formula and IMS profiling from TIMS-FT-ICR MS measurements. Identifications unique to t- 0 , t- 16 , and $\mathrm{t}-115 \mathrm{~h}$ are in black, red, and blue, respectively. When FT-ICR MS is used tracing the evolution of the chemical complexity is incomplete, because the isomeric complexity is not taken into account. Particularly, taking into account the isomeric variability indicates that the composition of the WAF at $\mathrm{t}-115 \mathrm{~h}$ is significantly more complex, and chemically unique, than is observed by FT-ICR MS alone. 
CHAPTER I

INTRODUCTION 


\subsection{Background}

Chemistry is a branch of physical science that deals with matter and how that matter changes. The discipline interacts with the world around us, focusing on the molecular level, where we seek to understand how molecules interact and how they change at an atomistic and molecular level. One of the key pieces to understanding matter is knowledge of a compounds chemical structure, which can be related to the properties of the molecule, and how it interacts and reacts with other molecules. At the close of the $19^{\text {th }}$ century, two of the most crucial developments in chemistry occurred, which would reshape our understanding of atomic structure: J.J. Thomson's 1897 discovery of the electron, ${ }^{1}$ followed by Wilhem Weins 1898 discovery that the path of positive ions could be deflected using a magnetic field. ${ }^{2}$ Their work not only led to greater understanding of the structure of the atom, but also showed that ions could be separated using their mass-to-charge ratio $(\mathrm{m} / \mathrm{z})$. In $1912 \mathrm{~J} . \mathrm{J}$ Thomson, together with Francis W. Aston, furthered Weins' work, establishing the field of mass spectrometry (MS), earning Aston the 1922 Nobel prize for the discovery of stable isotopes. $^{3}$

For many years mass spectrometry was the domain of physics, with X-ray crystallography being the method of choice for structural elucidation. However, some analytical and organic chemists, most notably Fred McLafferty and Klaus Biemann, saw the potential of mass spectrometry techniques to separate, quantify, and elucidate

molecular structure. ${ }^{4}$ Mclafferty and Biemann's work promoted mass spectrometry as a fundamental tool in analytical chemistry, through the development of new instrumentation and understanding how a mass spectrum, in particular the fragments, could be utilized to 
understand molecular structure. One challenge that was recognized early in the development of mass spectrometry was the difficulty associated with low resolving power instrumentation and the harshness associated with electron ionization, which produced a very complex fragmentation spectrum that required pre-separation of the molecular components of interest prior to MS analysis. In 1955 McLafferty and Gohlke at Dow Chemical Company were the first to interface a gas chromatography (GC) system to a Time of Flight (ToF) mass spectrometer, achieving separation of methanol, acetone, benzene, and toluene. ${ }^{5}$ Developments in GC-MS instrumentation resulted in it becoming the gold standard for separation and quantification of molecules of interest, including environmental contaminants such as polyaromatic hydrocarbons (PAHs) and chlorinated dioxins, hormones, drugs of abuse, petroleum, ${ }^{6}$ and has even found its way into outerspace, like the Huygens probe that landed on Titan. ${ }^{7}$

Over time, significant advances in mass spectrometry instrumentation have taken place, notably the development of the triple quadrupole (QqQ) by Richard Yost and Christie Enke in 1978, enabling molecular isolation, fragmentation, and mass analysis of the fragments, and ultra-high mass resolution mass spectrometry, with Fourier Transform Ion Cyclotron Resonance Mass Spectrometry (FT-ICR MS), developed by Alan Marshall and Melvin Comisarow in $1974 .{ }^{8,9}$ Advanced forms of mass spectrometry, such as FT-ICR MS, are able to separate an extraordinary number of molecular components using their mass-to-charge. In one particular example, over 86,000 mass spectral peaks, representing thousands of chemical formulas and their isotopes, were measured from petroleum. ${ }^{10}$ 
As more versatile forms of mass spectrometry instrumentation were developed, problems of greater complexity began to be addressed. In particular the development of electrospray ionization $(\mathrm{ESI})^{11}$ and matrix assisted laser desorption ionization (MALDI) ${ }^{12,13}$ greatly expanded the applicability of mass spectrometry to the analysis of labile, polar, and very large molecules, such as proteins, that were inaccessible by previous methodologies. The development of ESI made coupling high-performance-liquid chromatography (HPLC) systems to MS possible, thereby increasing the separation, identification, and quantification potential of the analysis. Liquid chromatography mass spectrometry (LC-MS) systems have been extremely successful in the analysis of molecules that are typically inaccessible by GC, with applications in clinical mass spectrometry,${ }^{14}$ proteomics and metabolomics,${ }^{15}$ analysis of drugs of abuse, ${ }^{16}$ and natural products. ${ }^{17}$

The separation of molecules in gas and liquid chromatography relies on the interaction of the analyte with the stationary phase of the column that is used, which retains the molecule until the correct temperature, as in GC, or solvent composition, as in LC, allows the molecule to be eluted. There are many structural considerations that affect the partitioning of a molecule to the stationary phase, and it is difficult to a posteriori determine the structure of a molecule on the basis of retention. A strength of GC-MS and LC-MS is the ability to identify and quantify a molecule utilizing isotopically labeled standards, which have the same elution properties of unlabeled standards. This advantage is also a limitation, that a known standard is required in order to understand the structure of an unknown molecule. 
In an alternative approach, ions can be separated based on their size by accelerating them with an electric field in a liquid or a gas, resulting in ions of different sizes having different velocities, or mobilities (K). The phenomenon of ion separation in the gas phase by size is known as ion mobility spectrometry (IMS), and was first described by John Zeleny, working with J.J. Thomson, in $1898 .{ }^{18}$ The principle of separation was refined by Arthur M. Tyndall, as a result of his research on the effect of gas composition, ${ }^{19}$ novel ion selection schemes, ${ }^{20}$ and the mobilities of pure substances. ${ }^{21}$ Similarly, ions in solution can also be separated by their size, a technique termed electrophoresis, and is a standard tool used for studying large biomolecules, such as DNA and proteins. Ion mobility spectrometry was first coupled to mass spectrometry in 1960 by Earl W. McDaniel, who combined a drift tube ion mobility spectrometer with a magnetic sector mass spectrometer in order to analyze ion-gas reactions of hydrogen. ${ }^{22,23}$ As IMS matured as a technique, various forms of instrumentation were developed such as drift-tube IMS, traveling wave ion mobility spectrometry (TWIMS), field asymmetric ion mobility spectrometry (FAIMS), and the differential mobility analyzer (DMA). ${ }^{24}$ IMS has found numerous applications in fieldwork, such as the analysis of drugs, explosives, and chemical warfare agents, primarily because the instruments can be miniaturized and used as handheld sensors. ${ }^{25}$

In IMS, separation is obtained as an ion is accelerated by an electric field, and experiences collisions with a bath gas, based on the collision cross section (CCS) of the ion, which depends on the structure of the ion and the bath gas, as shown by McDaniel and Mason. ${ }^{26}$ The CCS has been found to be a crucial tool in the structural elucidation of biomolecules, such as proteins and protein complexes, DNA, drugs, and hormones. In 
particular, IMS-MS has advantages for structural biology..$^{27,28}$ It has allowed researchers to answer fundamental questions including protein folding, ${ }^{29}$ especially for intrinsically disordered proteins, ${ }^{30}$ which cannot be crystallized and studies using X-ray crystallography. IMS has also been utilized in small molecule applications, such as in the study of pharmaceuticals, metabolites, and pesticides. ${ }^{31}$

In 2011, Francisco Fernandez-Lima and Melvin A. Park developed Trapped Ion Mobility Spectrometry (TIMS), which routinely exhibited resolving powers over 200. ${ }^{32-34}$ The principles of operation resemble the parallel flow instrument originally developed by Zeleny, ${ }^{18}$ where ions are pushed forward by a moving gas through a series of segmented ring electrodes where the electrodes have an increasing electric field gradient that retards the motion of the ions through the analyzer region. The ions reach an equilibrium between the moving gas colliding with the molecule, and the electric field, and are eluted into ion mobility resolved packets by reducing the electric field. In TIMS the ions are trapped within the device, and elution is a time-independent process that is regulated by the change in voltage gradient applied to the device. ${ }^{32-34} \mathrm{By}$ decreasing the rate of ion elution, resolving powers of up to 400 have been recorded for the analysis of polybrominated diphenyl ether metabolites. ${ }^{35}$ TIMS has a number of applications, including studying isomerization kinetics of small molecules,${ }^{36}$ peptides, DNA, proteins and their complexes in the absence of the bulk solvent, ${ }^{37-41}$ the influence of the collision partner on the molecular structure, ${ }^{42}$ and the factors that affect molecular-adduct complex lifetime and stability during TIMS measurements. ${ }^{43}$ 


\subsection{State of the Art Separations}

Mass spectrometry has found applicability and usage in a wide variety of applications, from basic science research on the catalytic properties of $5 \mathrm{~d}$ transition metals, ${ }^{44}$ to analysis of monoclonal antibodies in human serum, ${ }^{45}$ and elucidating drug metabolites in wastewater. ${ }^{46}$ As has been previously observed, as the scope of the analytical question increases in complexity, equivalent increases in instrumentation are required to solve the analytical challenge. This is the case in the analysis of unknown molecular species and complex mixtures, such as dissolved organic matter, ${ }^{47}$ petroleum, ${ }^{48}$ and complex biological samples of peptides and proteins. ${ }^{49}$ There are two challenges to overcome when approaching a complex mixture problem; first appears in the separation of the mass components, to be able to resolve them by MS, and the second is in separation of isomers and elucidation of the chemical structure. In modern applications, LC is typically utilized for molecular separations, enabling the use of instrumentation with low to high resolving power (nominal mass - 30,000). However, for complex biological, environmental, and petroleomic applications ultra-high mass resolution is required because of the complexity of the mass spectra and the inability to separate isobaric species (chemical compositions, including isotopes, with the same nominal mass). ${ }^{50}$ These applications have led to advances in instrumentation with ultra-high resolution, such as FT-ICR MS, increasing the magnetic fields up to $21 \mathrm{~T},{ }^{45}$ and implementing novel ICR cell designs. ${ }^{51-53}$

Addressing the challenge of structural elucidation for unknown structures represents a significant limitation for traditional GC-MS and LC-MS platform because the retention cannot be directly related to the structure of the molecule. However, in IMS the 
collision cross-section is related directly to the tri-dimensional structure of the molecule, enabling the separation of isomers and conformers in the analysis. ${ }^{31}$ Typically IMS analyzers are coupled to ToF MS because of the compatibility of the timescales for each analysis. ${ }^{24}$ However, given that ToF does not have sufficient resolving power for complex mixtures, limited attempts have been made to couple IMS and FT-ICR MS, such as the platform build by Bluhm et al. for the analysis of $\mathrm{Ar}^{+}$and $\mathrm{CO}^{+},{ }^{54}$ and a dual gate drift tube developed Tang et al. for the separation of isomeric phosphopeptides. ${ }^{55}$ As such, this work focuses on the development of a novel TIMS-FT-ICR MS platform, combining high ion mobility resolving power and ultra-high mass resolution.

\subsection{Dissertation Structure}

The subsequent seven chapters in this dissertation have been previously published and will explore the advantages of TIMS and describe the coupling of TIMS with FT-ICR MS. In chapter two, we describe the first coupling of Gas Chromatography to FT-ICR MS utilizing an Atmospheric Pressure Laser Ionization (APLI) source for the analysis of complex mixtures. The advantages of GC separation offered for the analysis of three crude oils were discussed and compared to analysis by direct infusion. We also describe the challenges faced by FTMS instrumentation when coupled to pre-separation techniques due to the long acquisition scan times of FT-ICR MS. ${ }^{56}$ Chapter three illustrates the use of TIMS for the analysis of PAHs in soil. In this work, we reported the collision cross-sections in nitrogen for 23 PAHs and how TIMS can be utilized to separate isomeric mixtures of multiple PAHs. We also reported on the theoretical methods used to calculate the CCS of the different standards and found good agreement $\left(<5 \%\right.$ deviation). ${ }^{57}$ The TIMS-MS 
workflow was expanded to other petrochemical substances in chapter four, with the analysis of metal octaethylporphyrins (OEP), which are analogues of geochemical markers for petroleum and shale oils. We observed in this work how upon metal binding, a significant shift in mass is observed while the CCS of the OEP stays largely similar to the unchelated sample. We also introduced the use of theoretical methods, such as Diffuse Hard Sphere Scattering for calculating the CCS of metal chelated molecules. ${ }^{58}$

In chapter five we proposed a workflow for structural assignment based on TIMSFT-ICR MS datasets, describing the generation, optimization, and calculation of structures, and how the experimental measurement of an ions mass and mobility can be utilized to assign candidate structures to PAHs.$^{59}$ In chapter six, we describe the first coupling of a variant of TIMS, Selected Accumulation Trapped Ion Mobility Spectrometry (SA-TIMS), to FT-ICR MS. In particular, we show how SA-TIMS can be utilized to separate endocrine disruptors from a complex mixture of dissolved organic matter, and how the use of known standards, or computational tools can be utilized to identify structural and cis/trans isomers in the gas phase. Of note is how the SA-TIMS analysis is a time independent process, which enabled 4-8s transients to be acquired per each mass spectrum, resulting in mass resolving powers up to 730,000 at $\mathrm{m} / \mathrm{z} 400 .^{60}$ This work was followed by chapter seven, where we described the fundamentals of SA-TIMS analysis and introduced the concept of oversampling to the TIMS analysis. This enabled the analytical characterization of structural isomers and conformational isomers of different peptides. We also presented an automated processing tool and how it can be applied to the analysis of complex data sets, 
enabling IMS-MS deconvolution of thousands of molecular signatures, enabling the generation of complex datasets based on $[\mathrm{m} / \mathrm{z}$; chemical formula; $\mathrm{K} ; \mathrm{CCS}]{ }^{61}$

The final chapter (8) is an application study of the TIMS-FT-ICR MS platform for the analysis of crude oil-water mixtures, and how the composition of water changes significantly as it is exposed to light. We show how the TIMS-FT-ICR MS platform is able to identify over 47,000 chemical features in a complex IMS-MS spectrum. We illustrate the high resolving power of TIMS-FT-ICR MS, and how this platform is ideal for the characterization of ultra-complex mixtures that requires high resolving power ion mobility separation, and ultra-high mass resolution. ${ }^{62}$ In the appendices can be found all of the supplementary information for each publication.

\subsection{References}

1.Thomson, J. J. Philos Mag (Abingdon) 1897, 44, 293-316.

2.Wien, W. Annalen der Physik und Chemie 1898, 65, 1-17.

3.Aston, F. W. Science Progress in the Twentieth Century (1906-1916) 1912, 7, 4865.

4.Griffiths, J. Anal. Chem., 2008, 80 (15), pp 5678-5683.

5.Gohlke, R. S.; McLafferty, F. W. J Am Soc Mass Spectrom 1993, 4, 367-371.

6.Hübschmann, H.-J. Handbook of GC-MS: fundamentals and applications; John Wiley \& Sons, 2015.

7.Niemann, H.; Atreya, S.; Bauer, S.; Carignan, G.; Demick, J.; Frost, R.; Gautier, D.; Haberman, J.; Harpold, D.; Hunten, D. Nature 2005, 438, 779-784.

8.Comisarow, M. B.; Marshall, A. G. Chem Phys Lett 1974, 25, 282-283.

9.Comisarow, M. B.; Marshall, A. G. J Mass Spectrom 1996, 31, 581-585. 
10. McKenna, A. M.; Williams, J. T.; Putman, J. C.; Aeppli, C.; Reddy, C. M.; Valentine, D. L.; Lemkau, K. L.; Kellermann, M. Y.; Savory, J. J.; Kaiser, N. K.; Marshall, A. G.; Rodgers, R. P. Energy Fuels 2014, 28, 2454-2464.

71.

11. Whitehouse, C. M.; Dreyer, R.; Yamashita, M.; Fenn, J. Science 1989, 246, 64-

12. Karas, M.; Bachmann, D.; Bahr, U. e.; Hillenkamp, F. Int J Mass Spectrom Ion Process 1987, 78, 53-68.

13. Tanaka, K.; Waki, H.; Ido, Y.; Akita, S.; Yoshida, Y.; Yoshida, T.; Matsuo, T. Rapid Commun Mass Spectrom 1988, 2, 151-153.

14. Chace, D. H.; DiPerna, J. C.; Naylor, E. W. Acta Pædiatrica 1999, 88, 45-47.

15. Zhou, B.; Xiao, J. F.; Tuli, L.; Ressom, H. W. Mol BioSyst 2012, 8, 470-481.

16. Pitt, J. J. Clin Biochem Rev 2009, 30, 19-34.

17. Přichystal, J.; Schug, K. A.; Lemr, K.; Novák, J.; Havlíček, V. Anal Chem 2016, 88, 10338-10346.

18. Zeleny, J. Philosophical Magazine 1898, 46, 120-154.

19. Tyndall, A. M.; Phillips, L. R. Proc Royal Soc A 1926, 111, 577-591.

20. Tyndall, A. M.; Starr, L. H.; Powell, C. F. Proc Royal Soc A 1928, 121, $172-$ 184.

21. Tyndall, A.; Powell, C. Proc Royal Soc A 1930, 129, 162-180.

22. McDaniel, E. W.; Martin, D. W.; Barnes, W. S. Rev Sci Instrum 1962, 33, 2-7.

23. Barnes, W. S.; Martin, D. W.; McDaniel, E. W. Phys Rev Let 1961, 6, 110-111.

24. May, J. C.; McLean, J. A. Anal Chem 2015, 87, 1422-1436.

25. Eiceman, G. A. Trends Anal Chem 21.4 (2002): 259-275.

26. McDaniel, E. W.; Mason, E. A. Mobility and diffusion of ions in gases; John Wiley and Sons, Inc., New York: New York, 1973, p 381.

27. Marklund, Erik G.; Degiacomi, Matteo T.; Robinson, Carol V.; Baldwin, Andrew J.; Benesch, Justin L. P. Structure 2015, 23, 791-799.

28. Uetrecht, C.; Barbu, I. M.; Shoemaker, G. K.; van Duijn, E.; HeckAlbert, J. R. Nat Chem 2011, 3, 126-132. 
29. Jurneczko, E.; Barran, P. E. Analyst 2011, 136, 20-28.

30. Jurneczko, E.; Cruickshank, F.; Porrini, M.; Nikolova, P.; Campuzano, Iain D. G.; Morris, M.; Barran, Perdita E. Biochem Soc Trans 2012, 40, 1021-1026.

31. Lapthorn, C.; Pullen, F.; Chowdhry, B. Z. Mass Spectrom Rev 2013, 32, 43-71.

32. Hernandez, D. R.; Debord, J. D.; Ridgeway, M. E.; Kaplan, D. A.; Park, M. A.; Fernandez-Lima, F. Analyst 2014, 139, 1913-1921.

33. Fernandez-Lima, F. A.; Kaplan, D. A.; Park, M. A. Rev Sci Instrum 2011, 82, 126106.

34. Fernandez-Lima, F.; Kaplan, D. A.; Suetering, J.; Park, M. A. Int J Ion Mobil Spectrom 2011, 14, 93-98.

35. Adams, K. J.; Montero, D.; Aga, D.; Fernandez-Lima, F. Int J Ion Mobil Spectrom 2016, 19, 69-76.

36. Schenk, E. R.; Mendez, V.; Landrum, J. T.; Ridgeway, M. E.; Park, M. A.; Fernandez-Lima, F. Anal Chem 2014, 86, 2019-2024.

37. Schenk, E. R.; Ridgeway, M. E.; Park, M. A.; Leng, F.; Fernandez-Lima, F. Anal Chem 2014, 86, 1210-1214.

38. Schenk, E. R.; Nau, F.; Fernandez-Lima, F. Int J Ion Mobil Spectrom 2015, 18, 23-29.

39. Schenk, E. R.; Almeida, R.; Miksovska, J.; Ridgeway, M. E.; Park, M. A.; Fernandez-Lima, F. J Am Soc Mass Spectrom 2015, 26, 555-563.

40. Benigni, P.; Marin, R.; Molano-Arevalo, J. C.; Garabedian, A.; Wolff, J. J.; Ridgeway, M. E.; Park, M. A.; Fernandez-Lima, F. Int J Ion Mobil Spectrom 2016, 19, 95104.

41. Garabedian, A.; Butcher, D.; Lippens, J. L.; Miksovska, J.; Chapagain, P. P.; Fabris, D.; Ridgeway, M. E.; Park, M. A.; Fernandez-Lima, F. Phys Chem Chem Phys 2016, 18, 26691-26702.

42. Molano-Arevalo, J. C.; Hernandez, D. R.; Gonzalez, W. G.; Miksovska, J.; Ridgeway, M. E.; Park, M. A.; Fernandez-Lima, F. Anal Chem 2014, 86, 10223-10230.

43. McKenzie-Coe, A.; DeBord, J. D.; Ridgeway, M.; Park, M.; Eiceman, G.; Fernandez-Lima, F. Analyst 2015, 140, 5692-5699.

44. Armentrout, P. B. Chem Eur J 2017, 23, 10-18. 
45. He, L.; Anderson, L. C.; Barnidge, D. R.; Murray, D. L.; Hendrickson, C. L.; Marshall, A. G. J Am Soc Mass Spectrom 2017, 5, 827-838.

46. Wang, J.; Gardinali, P. R. Chemosphere 2014, 107, 65-73.

47. Hertkorn, N.; Harir, M.; Cawley, K.; Schmitt-Kopplin, P.; Jaffé, R. Biogeosciences Discus 2015, 12.

48. Rodgers, R. P.; Schaub, T. M.; Marshall, A. G. Anal Chem 2005, 77, 20 A-27 A.

49. Washburn, M. P.; Wolters, D.; Yates, J. R. Nature biotechno 2001, 19, 242-247.

50. Marshall, A. G.; Rodgers, R. P. Acc Chem Res 2004, 37, 53-59.

51. Kostyukevich, Y. I.; Vladimirov, G. N.; Nikolaev, E. N. J Am Soc Mass Spectrom 2012, 23, 2198-2207.

52. Nagornov, K. O.; Kozhinov, A. N.; Tsybin, O. Y.; Tsybin, Y. O. J Am Soc Mass Spectrom 2015, 26, 741-751.

53. Popov, I. A.; Nagornov, K.; Vladimirov, G. N.; Kostyukevich, Y. I.; Nikolaev, E. N. J Am Soc Mass Spectrom 2014, 25, 790-799. 4086.

54. Bluhm, B. K.; Gillig, K. J.; Russell, D. H. Rev Sci Instrum 2000, 71, 4078-

55. Tang, X.; Bruce, J. E.; Hill, H. H., Jr. Rapid Commun Mass Spectrom 2007, 21, $1115-1122$.

56. Benigni, P.; DeBord, J. D.; Thompson, C. J.; Gardinali, P.; Fernandez-Lima, F. Energy Fuels 2016, 30, 196-203.

57. Castellanos, A.; Benigni, P.; Hernandez, D. R.; DeBord, J. D.; Ridgeway, M. E.; Park, M. A.; Fernandez-Lima, F. Anal Meth 2014, 6, 9328-9332.

58. Paolo Benigni, C. B., J. Martin E. Quirke, John Daniel DeBord, Alexander Moiseevich Mebel,; Fernandez-Lima, F. Energy Fuels 2016.

59. Benigni, P.; Marin, R.; Fernandez-Lima, F. Int J Ion Mobil Spectro 2015, 18, 151-157.

60. Benigni, P.; Thompson, C. J.; Ridgeway, M. E.; Park, M. A.; Fernandez-Lima, F. Anal Chem 2015, 87, 4321-4325.

61. Benigni, P.; Fernandez-Lima, F. Anal Chem 2016, 88, 7404-7412. 
62. Benigni, P.; Sandoval, K.; Thompson, C. J.; Ridgeway, M. E.; Park, M. A.; Gardinali, P. R.; Fernandez-Lima, F. Environ Sci Technol 2017. 
CHAPTER II

INCREASING POLYAROMATIC HYDROCARBON (PAH) MOLECULAR COVERAGE DURING FOSSIL OIL ANALYSIS BY COMBINING GAS CHROMATOGRAPHY AND ATMOSPHERIC-PRESSURE LASER IONIZATION FOURIER TRANSFORM ION CYCLOTRON RESONANCE MASS SPECTROMETRY (FT-ICR MS)

(Adapted with permission from Benigni et al., 2015, Energy and Fuels, copyright 2017 American Chemical Society) 


\subsection{Abstract}

Thousands of chemically distinct compounds are encountered in fossil oil samples that require rapid screening and accurate identification. In the present paper, we show for the first time, the advantages of gas chromatography (GC) separation in combination with atmospheric-pressure laser ionization (APLI) and ultrahigh-resolution Fourier transform ion cyclotron resonance mass spectrometry (FT-ICR MS) for the screening of polyaromatic hydrocarbons (PAHs) in fossil oils. In particular, reference standards of organics in shale oil, petroleum crude oil, and heavy sweet crude oil were characterized by GC-APLI-FTICR MS and APLI-FT-ICR MS. Results showed that, while APLI increases the ionization efficiency of PAHs, when compared to other ionization sources, the complexity of the fossil oils reduces the probability of ionizing lower-concentration compounds during direct infusion. When gas chromatography precedes APLI-FT-ICR MS, an increase (more than 2-fold) in the ionization efficiency and an increase in the signal-to-noise ratio of lowerconcentration fractions are observed, giving better molecular coverage in the $m / z$ 100-450 range. That is, the use of GC prior to APLI-FT-ICR MS resulted in higher molecular coverage, higher sensitivity, and the ability to separate and characterize molecular isomers, while maintaining the ultrahigh resolution and mass accuracy of the FT-ICR MS separation.

\subsection{Introduction}

While bulk features of fossil oils can be resolved using infrared and near-infrared spectroscopy, molecular component characterization is traditionally limited to mass spectrometry (MS) based techniques (more details are given in refs 1 and 2). Over the last decades, multiple MS-hyphenated techniques have been successfully applied to the 
characterization of fossil oils (e.g., gas chromatography-mass spectrometry (GC-MS), 3,4 two-dimensional gas chromatography-mass spectrometry (2D GCMS) ${ }^{5}$ liquid chromatography-mass spectrometry (LC-MS), ${ }^{6,7}$ and, more recently, ion mobility spectrometry-mass spectrometry (IMS-MS ${ }^{8-13}$ ). In particular, the advantages of Fourier transform ion cyclotron resonance mass spectroscopy (FT-ICR MS) analyzers have been previously described for the identification of a large number of chemical components during a single analysis of fossil oils using high mass accuracy and ultrahigh mass resolution..$^{14-18}$

With the development of atmospheric-pressure ionization (API) sources, multiple studies have shown unique advantages for the characterization of fossil oils targeting different functional groups, aromatic content, and polarity (e.g., electrospray ionization (ESI) ${ }_{19}^{19}$ atmospheric-pressure photo ionization (APPI) ${ }^{20,21}$ atmospheric-pressure chemical ionization (APCI), ${ }^{22-24}$ atmospheric-pressure laser ionization (APLI), ${ }^{25-28}$ laser desorption ionization (LDI) ${ }^{29-31}$ direct analysis in real time (DART), ${ }^{32,33}$ desorption electrospray ionization (DESI), ${ }^{34}$ laser-induced acoustic desorption electron impact (LIAD-EI),${ }^{35}$ laserinduced acoustic desorption chemical ionization (LIAD-CI), ${ }^{36}$ and low-temperature plasma $\left.(\mathrm{LTP})^{37}\right)$. While prior studies have described the coupling of chromatographic separations with electron impact sources (e.g., GC-EI-TOF-MS, ${ }^{38}$ GC-EI-QLT-Orbitrap, ${ }^{39}$ and GC-EIFTICR $M S^{40,41}$ ), more recent has shown advantages of coupling GC and LC to API-FTICR MS for the detection of molecular components and the separation of isomeric components (e.g., GC-APCI-FT-ICR MS, ${ }^{15,42}$ and HPLC-ESI/APCI/APPI/APLI-FT-ICR $\left.\mathrm{MS}^{43,44}\right)$. 
The work described herein focuses on the analysis of PAHs from fossil oils using APLI and ultrahigh-resolution FT-ICR MS spectrometry (APLI-FT-ICR MS). Previous studies have shown that, compared to APCI and APPI, APLI is more suitable for the characterization of conjugated PAHs with increased sensitivity and selective ionization of highly conjugated compounds using lower-resolution MS analyzers. ${ }^{27,45-47}$

Results will show, for the first time, the advantages of combining GC separation and APLI-FT-ICR MS for the screening of PAHs in fossil oils (GC-APLI-FT-ICR MS). The screening potential of GC-APLI-FT-ICR MS is illustrated with the analysis of three reference fossil oil standards: organics in shale oil (OSO), petroleum crude oil (PCO), and heavy sweet crude oil (HSO). It will be shown that the use of retention time and accurate mass measurements for unambiguous identification of molecular components and structural assignments in complex mixtures has potential for targeted analysis and fingerprinting of lower-concentration fractions in the low mass range in fossil fuels.

\subsection{Experimental Section}

\subsubsection{Sample Preparation}

Standard reference materials of organics in shale oil (OSO, SRM 1580), petroleum crude oil (PCO, SRM 1582), and heavy sweet crude oil (HSO, SRM 2722) were obtained from the National Institute of Standards and Technology (Baltimore, MA) and used as received. More detailed information on the PAHs and alkyl-PAHs content for SRM $1580 / 1582$ and on the sulfur content $(0.21 \% \mathrm{wt} / \mathrm{wt})$ for SRM 2722 can be found in the

certificates ${ }^{48-50}$ Prior analysis, samples were diluted at 1:5, 1:10, and 1:100 (v/v) ratios for direct-infusion APLI and 1:100 (v/v) for GC-APLI in Optima-grade hexane (Fisher Scientific, Waltham, MA). 


\subsubsection{APLI-FT-ICR MS Analysis}

Individual standards were directly infused into a custom-built atmospheric-pressure laser ionization source (APLI) source using a vaporizer at a constant temperature of 300 ${ }^{\circ} \mathrm{C}$ at a rate of $200 \mu \mathrm{L} / \mathrm{h}$. (See details of the APLI source and coupling in Appendix 2.1) Details on the APLI principles of operation can be found elsewhere. ${ }^{27}$ Briefly, a $266 \mathrm{~nm}$ laser beam (CryLas GmbH, Berlin, Germany; Type 1HP266-50) is introduced orthogonal to the glass capillary source inlet of the 7T Solarix FT-ICR MS spectrometer (Bruker Daltonics, Inc., Billerica, MA). A molecular beam intercepts the laser beam and molecules are ionized via a two-photon $(1+1)$ ionization mechanism and introduced into a FT-ICR MS spectrometer ${ }^{51}$ Samples were analyzed in positive ion mode and ion transmission was optimized for the $m / z 100-900$ range. Ions were accumulated in the collision cell (2 MHz, $1000 \mathrm{Vpp}$ ) for $0.1 \mathrm{~s}$ during detection using "Accumulate During Detect" mode in order to reduce overall analysis time. FT-ICR MS spectra were acquired over 25 time domain acquisition at 4 MWord ( $2 \mathrm{~s}$ transient). FT-ICR signals were processed using a half-sine apodization followed by fast-Fourier transform and broadband phase correction (absorption spectra using absorption mode processing (AMP)), ${ }^{52,53}$ resulting in an $\sim 2$-fold increase in mass resolution (experimental MS resolving power with AMP at $m / z 400$ of 424,000).

\subsubsection{GC-APLI-FT-ICR MS Analysis}

Individual standards were separated using a custom-built gas chromatography (GC) and introduced to the APLI source via a GC transfer line heated to $300{ }^{\circ} \mathrm{C}$ (more details on the GC-APLI coupling in Appendix 2.1). This source is now commercially available via Bruker Daltonics, Inc. GC separation was performed using a DB-5 Ms+DG column (30 m 
$\times 0.25 \mathrm{~mm}, 0.25 \mu \mathrm{m}$ thickness, from Agilent Technologies, Inc., Palo Alto, CA). The GC injection chamber was held at $200{ }^{\circ} \mathrm{C}$ and $1 \mu \mathrm{L}$ of sample was introduced at a 1:20 split ratio. The GC method consisted of a $110-230{ }^{\circ} \mathrm{C}$ ramp at a rate of $10{ }^{\circ} \mathrm{C} / \mathrm{min}$, followed by a $230-310{ }^{\circ} \mathrm{C}$ ramp at a rate of $5{ }^{\circ} \mathrm{C} / \mathrm{min}$, and held for $7 \mathrm{~min}$ for a total of $35 \mathrm{~min}$. FT-ICR MS spectra were acquired after $5 \mathrm{~min}$, for a total of $25 \mathrm{~min}$, with similar ion transmission conditions to those used during direct infusion APLI-FT-ICR MS but without averaging and with a shorter collection time of 2 MWord ( $1 \mathrm{~s}$ transient), resulting in an experimental MS resolving power with AMP at $m / z 400$ of 264,000 .

\subsubsection{Data Processing}

FT-ICR MS spectra were externally and internally calibrated using a Tuning Mix standard (Tunemix, G2421A, Agilent Technologies, Santa Clara, CA) ${ }^{53}$ and known PAH series, respectively. The peak lists were generated allowing for a $\mathrm{S} / \mathrm{N}$ ratio of 6 . For GCAPLI-generated data, the summed MS of all the scans was used to generate the peak list. The formulas calculations from the exact mass domain were performed using Composer software (Version 1.0.6, Sierra Analytics, CA) with a maximum formula of $\mathrm{C}_{1-100} \mathrm{H}_{1-100} \mathrm{~N}_{0-2} \mathrm{O}_{0-2} \mathrm{~S}_{0-2}$, odd and even electron configurations allowed, and a mass tolerance of $0.5 \mathrm{ppm}$. Two-dimensional GC-FT-ICR MS data were processed by generating the extracted ion chromatography (EIC) chromatograms for each chemical formula, using Data Analysis software (version 4.2SR2, Bruker Daltonics, Inc., Billerica, MA); peak detection (isomer contribution) on the EIC chromatogram was performed using custom MATLAB scripts with a peak criteria of 4 points across the peak and a minimum $20 \%$ intensity after smoothing using the Whittaker method (with $\lambda=6$ ). ${ }^{54}$ The double-bond 
equivalents (DBEs) versus carbon number and retention time versus carbon number plots were generated using MATLAB software (R2014b, MathWorks, Inc., Natick, MA).

\subsection{Results and Discussion}

The analysis of the OSO, PCO, and HSO fossil oils standards using APLI-FT-ICR MS can be characterized by broad distributions in the $m / z, 100-900$ range centered between

a)

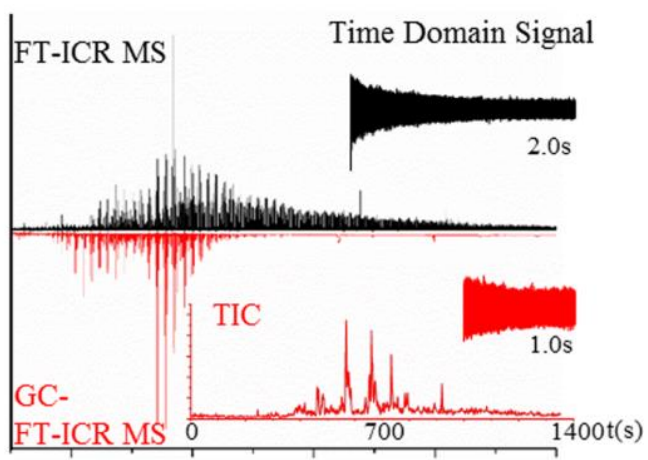

b)

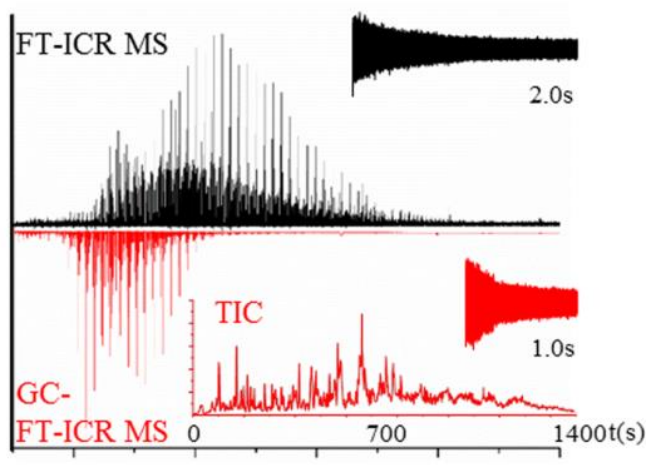

c)

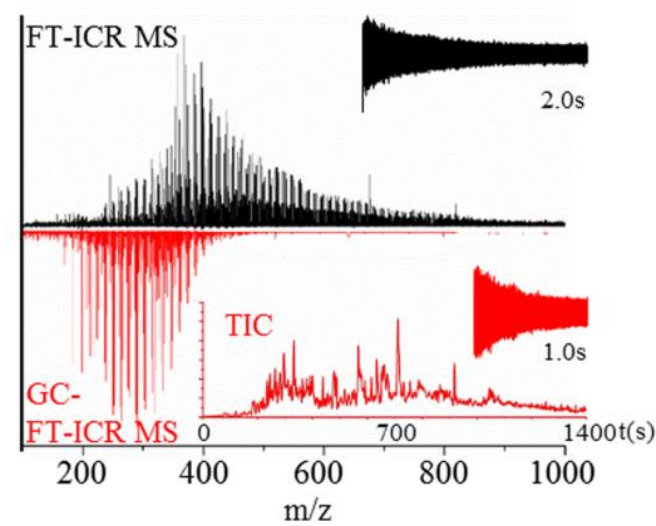

Figure 2.1. Typical FT-ICR MS spectra of (a) petroleum crude oil (PCO), (b) organics in shale oil (OSO), and (c) heavy sweet crude oil (HSO) obtained by direct-infusion APLI-FT-ICR MS (top, black spectrum) and GC-APLI-FT-ICR MS (bottom, red spectrum). Also shown is the time domain signal for the directinfusion analysis (black) and a single scan in the GC-FTMS analysis, along with the total ion chromatogram (red). 
$\mathrm{m} / z$ 400-500 (Figure 2.1, top black spectra). These broad distributions are in good agreement with previous studies of fossil oils using API sources (e.g., ESI, APCI, APPI, LDI). ${ }^{3,8,20,21,29,43,55-61}$ The broad distributions can be attributed to the large number of compounds, the chemical diversity, and the number of components per heteroatom PAH series commonly encountered in the fossil oils. The sum of all the individual mass spectrum of the GC-APLI-FT-ICR MS analysis (Figure 2.1, bottom red spectra) shows that, although the MS spectrum does not have the added benefit of multiple coadded transients (i.e., 25 added transients in the case of APLI-FT-ICR MS), the simplified ion population allows for sensitive detection and high mass accuracy using half of the transient time. Closer inspection shows that the GC-APLI-FTICR MS spectrum has a high mass cutoff at $m / z \approx$ 450 , as a consequence of the volatility range of compounds that are eluted from the GC separation. This mass cutoff results in large differences in the number of assignments when comparing between GC-APLI-FT-ICR MS and APLI-FT-ICR MS: 1734, compared to 6272 for the PCO; 1723, compared to 9188 for the OSO; and 1655, compared to 6216 for the HSO. The total number of unique chemical compounds identified from the combined GC-APLI-FT-ICR MS and APLI-FT-ICR MS analyses is 6758, 9700, and 7869, for the PCO, OSO, and HSO, respectively, consistent with typically observed numbers in fossil oils reports using APLI-FT-ICR MS. ${ }^{62}$ Taking advantage of the high resolving power and mass accuracy of the FT-ICR MS, chemical signatures were detected for the PCO, OSO, and HSO fossil oils based on the PAH classes and relative abundances (see Appendix 2.2). That is, inspection of the primary heteroatom PAH series (e.g., HC, N, O, and S) shows differences in the structural composition per class and in the relative compositions of PCO, 
HC Class

N Class

O Class

S Class

a)
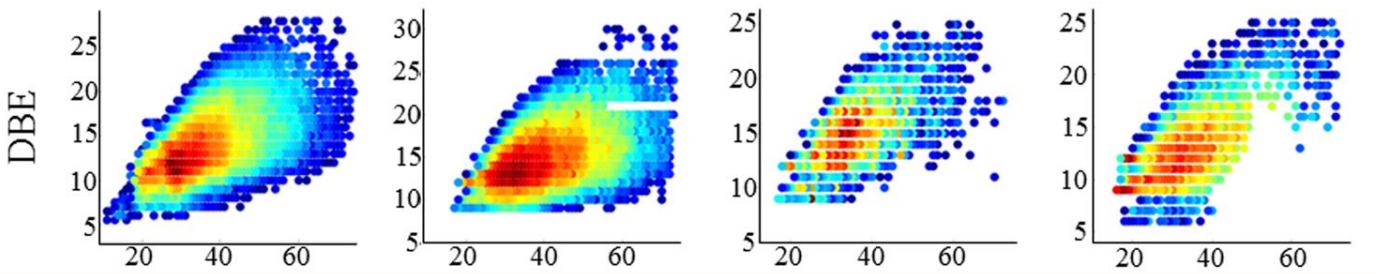

芳
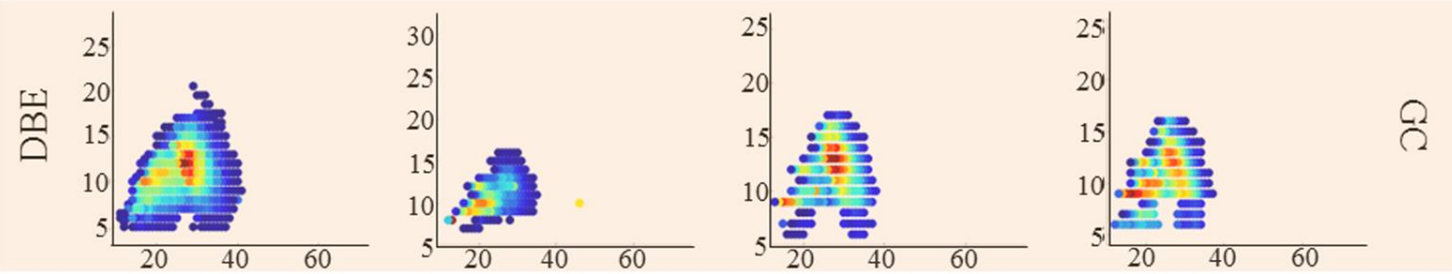

b)
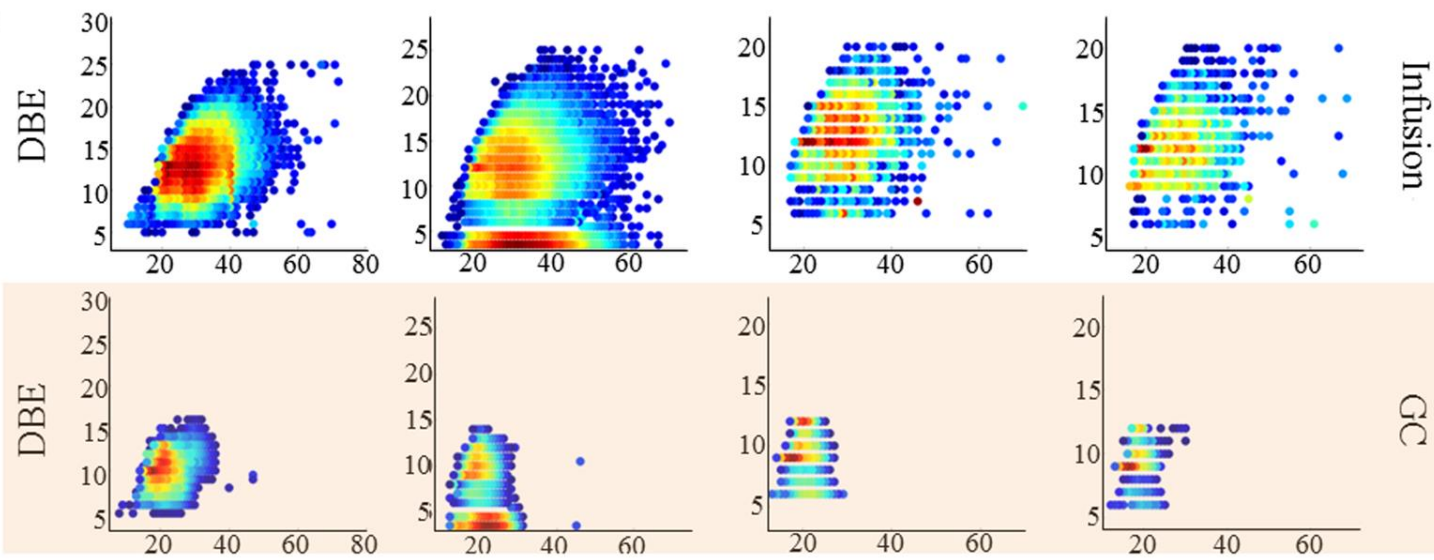

c)
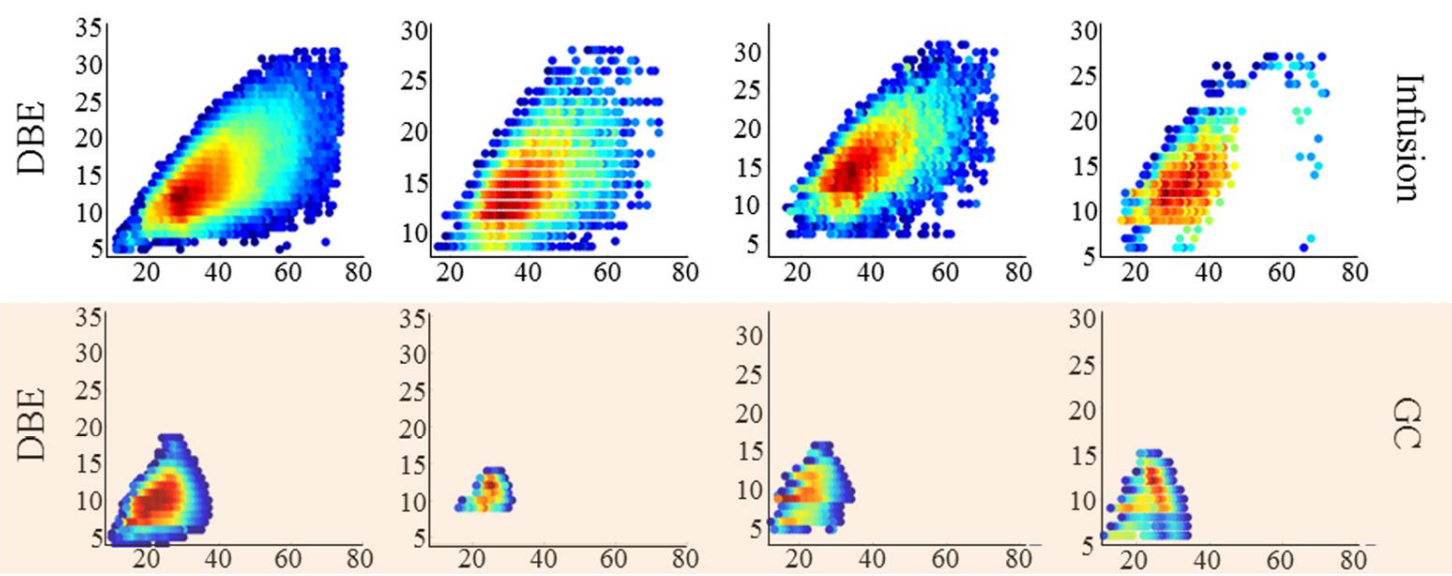

Carbon Number

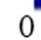

Figure 2.2. Double-bond equivalent (DBE) versus carbon number for the $\mathrm{HC}, \mathrm{N}, \mathrm{O}$ and $\mathrm{S}$ classes of (a) petroleum crude oil, (b) organics in shale oil (OSO), and (c) heavy sweet oil (HSO) obtained by directinfusion APLI-FT-ICR MS (top) and GC-APLI-FT-ICR MS (bottom).

OSO, and HSO fossil oils (see Figures 2.2 and 2.3). For example, the PCO and HSO shows similar $\mathrm{HC}$ and $\mathrm{N}$ composition (typical aromatic limit for the ratio of $\mathrm{DBE}$ to carbon 
number ${ }^{63}$ ), in contrast to the $\mathrm{OSO}$ where the most intense series in the $\mathrm{N}$ class corresponds to DBE 3.5 and 4.5 (Figure 2.2). This OSO signature is indicative of a very intense pyrydinic series, ${ }^{64}$ as well as partially aromatic secondary amines series, which has been previously observed in shale oils from the Mahogany zone of the Green River Formation. ${ }^{65,66}$ In all cases, incomplete assignment of the $\mathrm{S}$ class in the higher mass range may be observed, because of insufficient resolving power.

The comparison of the number of compounds detected by GC-APLI-FT-ICR MS and APLI-FT-ICR MS shows that GC preseparation enhances the molecular coverage in the $m / z$ 100-450 range (Figure 2.3). That is, a total of 1928, 3900, and 2087 formulas were identified below $\mathrm{m} / \mathrm{z} 450$ from combined methods for the PCO, OSO, and HSO, respectively. However, 23\%, 13\%, and 27\% (corresponding to 436, 512, and 565 chemical formulas) of the identifications for the $\mathrm{PCO}$, OSO, and $\mathrm{HSO}$, respectively, were detected only when GC preseparation was used. The increase in the number of formula hits can be correlated by class, such as the $\sim 23 \%, \sim 10 \%$, or $25 \%$ increase that is observed for the $\mathrm{HC}$, $\mathrm{N}$, or $\mathrm{O} / \mathrm{S}$ classes. This enhancement is notable in the smaller mass and lower DBE species for the $\mathrm{HC}$ and $\mathrm{O}$ classes and may be related to ion suppression in the APLI source (see Appendix 2.3).

The main advantage of APLI sources for the analysis of PAHs is based on the selectivity for ionizing conjugated systems. ${ }^{1,21,25,26,29,45,55,67-70}$ That is, the use of APLI for the analysis of crude oils reduces the presence of common contaminants and interferences (e.g., GC column bleeding, source contamination, solvent impurities, etc.). ${ }^{43,44}$ However, the molecular ionization efficiency during APLI can be limited by (i) matrix effects and (ii) source brightness for the case of complex samples. A comparison between the GC- 


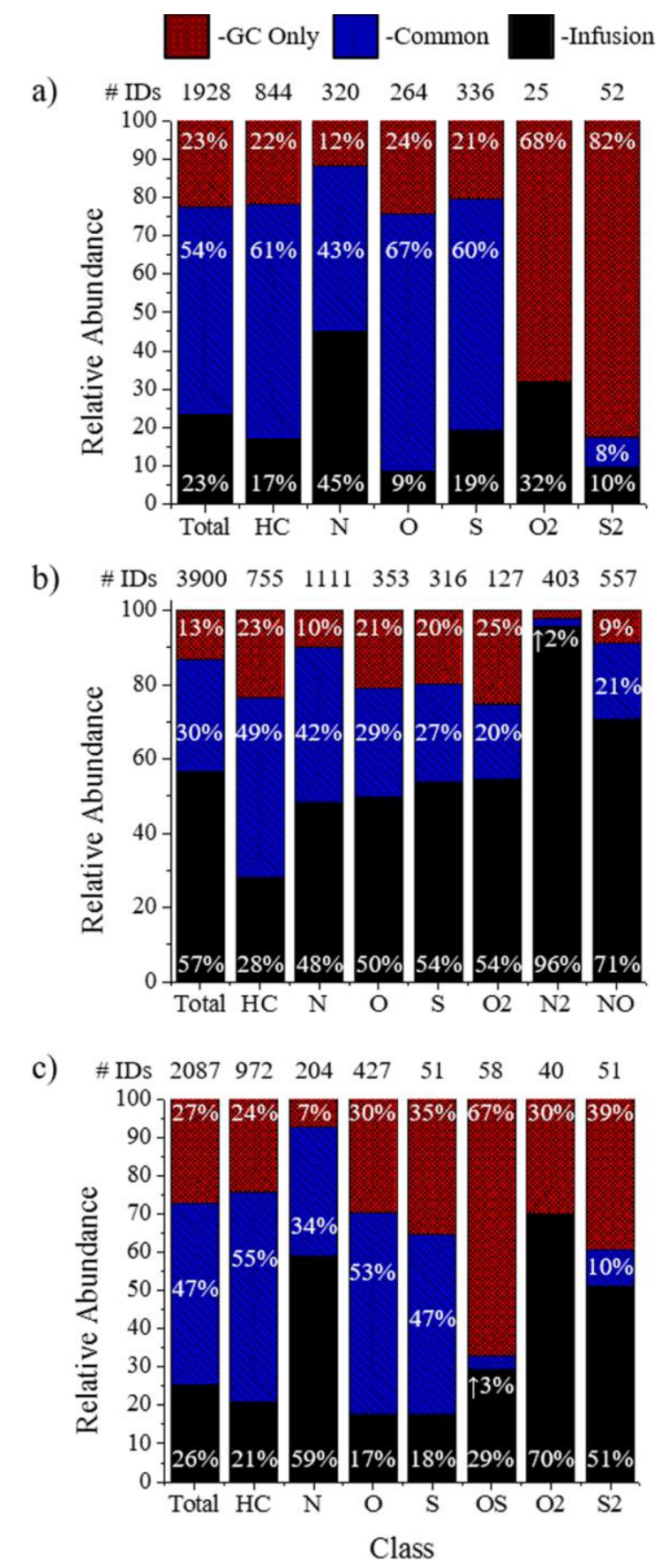

Figure 2.3. Percentages of compounds observed only by GC (GC only, red), common between both analysis (blue), and seen by infusion only (black) in the $\mathrm{m} / \mathrm{z}, 100-450$ range, compared to the total number of identifications, as a function of the heteroatom class in (a) petroleum crude oil (PCO), (b) organics in shale oil (OSO), and (c) heavy sweet oil (HSO).

APLI-FTICR MS and APLI-FT-ICR MS data shows that the reduction in complexity and preconcentration of the GC allows for higher sensitivity (Table 2.1). That is, lower concentrations can be detected with greater S/N, using GC-APLI-FT-ICR MS, when 
Table 2.1. Reported Compounds Observed by APLI-FT-ICR MS and GC-FT-ICR MS in the Organics in Shale Crude Oil and Petroleum Crude Oil Certificates

\begin{tabular}{|c|c|c|c|c|c|c|c|}
\hline \multirow[b]{2}{*}{ formula } & \multirow[b]{2}{*}[\mathrm{M}]{+} & \multirow[b]{2}{*}{ compound } & \multirow[b]{2}{*}{ certificate reference } & \multirow{2}{*}{$\frac{\mathrm{GC}}{1: 100}$} & \multicolumn{3}{|c|}{ Infusion } \\
\hline & & & & & $1: 100$ & $1: 10$ & 1:05 \\
\hline $\mathrm{C}_{14} \mathrm{H}_{10}$ & 178.0777 & phenanthrene & SRM 1582 & $x$ & & & \\
\hline $\mathrm{C}_{16} \mathrm{H}_{10}$ & 202.0777 & fluoranthene & SRM 1580/1582 & $x$ & $x$ & & \\
\hline $\mathrm{C}_{16} \mathrm{H}_{10}$ & 202.0777 & pyrene & SRM 1580/1582 & $x$ & $x$ & & \\
\hline $\mathrm{C}_{18} \mathrm{H}_{12}$ & 228.0933 & benzanthracene & SRM 1582 & $x$ & $x$ & & \\
\hline $\mathrm{C}_{20} \mathrm{H}_{12}$ & 252.0933 & benzopyrene & SRM 1580/1582 & $x$ & $x$ & $x$ & \\
\hline $\mathrm{C}_{20} \mathrm{H}_{12}$ & 252.0933 & perylene & SRM 1580/1582 & $x$ & $x$ & $x$ & \\
\hline $\mathrm{C}_{22} \mathrm{H}_{12}$ & 276.0933 & benzo(ghi)perylene & SRM 1582 & $x$ & $x$ & $\times$ & $\times$ \\
\hline $\mathrm{C}_{22} \mathrm{H}_{12}$ & 276.0933 & indeno[1,2,3-cd]pyrene & SRM 1582 & $x$ & $x$ & $x$ & $x$ \\
\hline $\mathrm{C}_{12} \mathrm{H}_{8} \mathrm{~S}$ & 184.0341 & dibenzothiophene & SRM 1582 & $x$ & & & \\
\hline
\end{tabular}
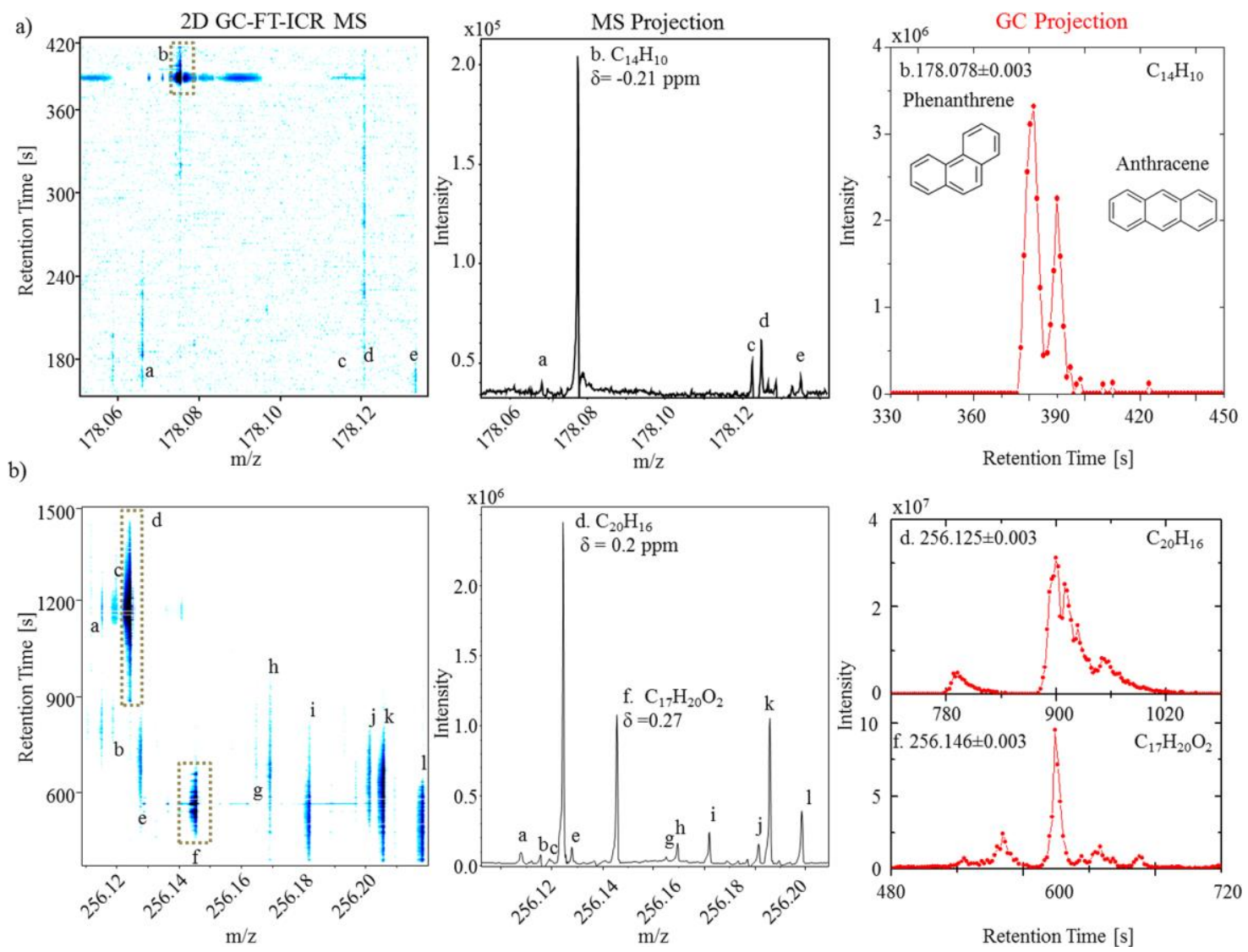

Figure 2.4. 2D GC-FT-ICR MS contour plots, mass spectrum projections, and selected GC traces for (a) $\mathrm{m} / \mathrm{z}$ 178 and (b) $\mathrm{m} / \mathrm{z} 256$ for the organics in shale oil (OSO). Peak assignments can be found in Appendix 2.6

compared to APLI-FT-ICR MS. The APLI-FT-ICR MS concentration experiment (e.g., 1:5, 1:10, and 1:100 dilutions) suggests that there are no matrix effects (see Appendix 2.4 and 2.5). These experiments showed that the disappearance of small molecules (below $\mathrm{m} / \mathrm{z}$ 


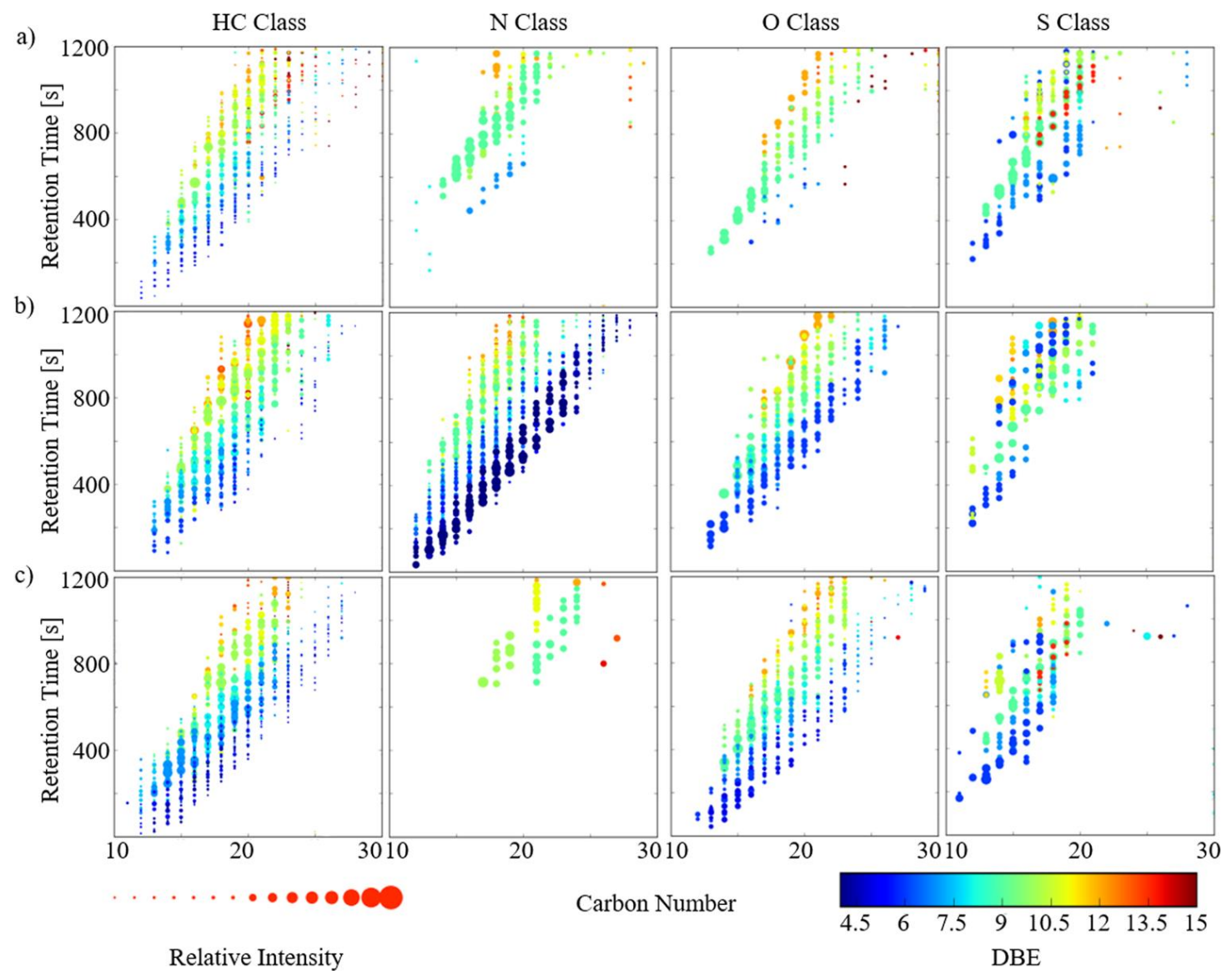

Figure 2.5. Two-dimensional (2D) GC-FT-ICR MS contour plots including the double-bond equivalent (DBE, color scale) and relative abundance (symbol size) for the primary heteroatom PAH classes in (a) petroleum crude oil (PCO), (b) organics in shale oil (OSO), and (c) heavy sweet oil (HSO).

200) in both sample types was not related to the initial sample concentration. These results suggest that the probability of ionizing by APLI is defined by the number of molecules present in the ionization region, the source fluence, and the photon absorption cross section of the molecule of interest. Other improvements in the detection of the low concentration molecules can be attributed to the reduction of the number of molecules within the ICR cell, thereby increasing the dynamic range. ${ }^{71}$

An analytical benefit of GC-APLI-FT-ICR MS, compared to APLI-FT-ICR MS, is the added potential to separate and identify molecular isomers. That is, ion chromatograms can be generated for targeted PAH compounds with reduced isobaric interferences when 
using GC-APLI-FT-ICR MS (Figure 2.4). For example, closer inspection of the 2D GCMS contour plot shows four molecular formulas and multiple isomers at $m / z, 178$ in the OSO analysis (see Figure 2.4a and Appendix 2.6). Inspection of the MS projection allows for the assignment of several compounds at the nominal mass level using the high mass accuracy. The most intense peak (labeled " $b$ " in Figure 2.4a) at $m / z 178$ corresponds to $\mathrm{C}_{14} \mathrm{H}_{10}($ error $=-0.21 \mathrm{ppm})$. Inspection of the $\mathrm{C}_{14} \mathrm{H}_{10}(\mathrm{~m} / z \pm 0.003)$ chromatogram shows the separation and presence of the molecular isomers phenanthrene and anthracene (previously validated using individual standard analysis). Note that both GC base peak widths obtained during the GC-APLI-FT-ICR MS are similar to those reported using traditional GC-MS separations of OSO SRM. ${ }^{48,72,73}$ That is, the use of APLI-FTICR MS did not compromise the GC separation or peak shape.

As the molecular mass increases a larger chemical and structural diversity is observed in the 2D GC-MS plots of fossil oils. For example, closer inspection at $m / z 256$ of OSO SRM shows multiple interferences that cannot be resolved by GC or MS alone. The MS analysis provided 12 molecular formulas with sub-ppm accuracy (labeled "a"-“l”" in Figure 2.4b; chemical formulas are described in Appendix 2.6). The high mass separation also permitted the generation of ion chromatograms without interferences per chemical formula (see, for example, $\mathrm{C}_{20} \mathrm{H}_{16}$ and $\mathrm{C}_{17} \mathrm{H}_{20} \mathrm{O}_{2}$ in Figure 2.4b). For example, the chromatogram for $\mathrm{C}_{20} \mathrm{H}_{16}$ can be generated without contribution from the other two overlapping $\mathrm{GC}$ signals and $\mathrm{C}_{17} \mathrm{H}_{20} \mathrm{O}_{2}$ from the seven overlapping signals. The $\mathrm{GC}$ projections $\mathrm{C}_{20} \mathrm{H}_{16}$ and $\mathrm{C}_{17} \mathrm{H}_{20} \mathrm{O}_{2}$ showed the presence of multiple isomers with GC peaks of $\sim 10 \mathrm{~s}$ at the base (corresponding to nine FT-ICR MS scans). 
An added benefit of the GC-APLI-FT-ICR MS analysis is the possibility to incorporate in the traditional 2D GC-MS plots the DBE information and relative abundances for a better comparison between fossil oils (see Figure 2.5 for the PCO, OSO, and HSO). That is, GC-MS (with DBE) plots allow for the visualization of signature trends between the fossil oils. For example, if the same GC-MS (with DBE) is plotted as a function of the carbon number per heteroatom class, a better illustration of the structural complexity is obtained within each class. As the retention time increases, an increase in the DBE value is observed per carbon number, in good agreement with previous GC-MS data. ${ }^{48,72,73}$ Inspection of the 2D GC-MS (with DBE) plots (Figure 2.5) clearly shows the main differences across the fossil oils. For example, differences in the $\mathrm{N}$ series at low DBE between the OSO, and the PCO and HSO, and in the $\mathrm{O}$ series between the PCO, and the OSO and HSO. For the case of targeted analysis, we anticipate that this plot will permit a facile visualization.

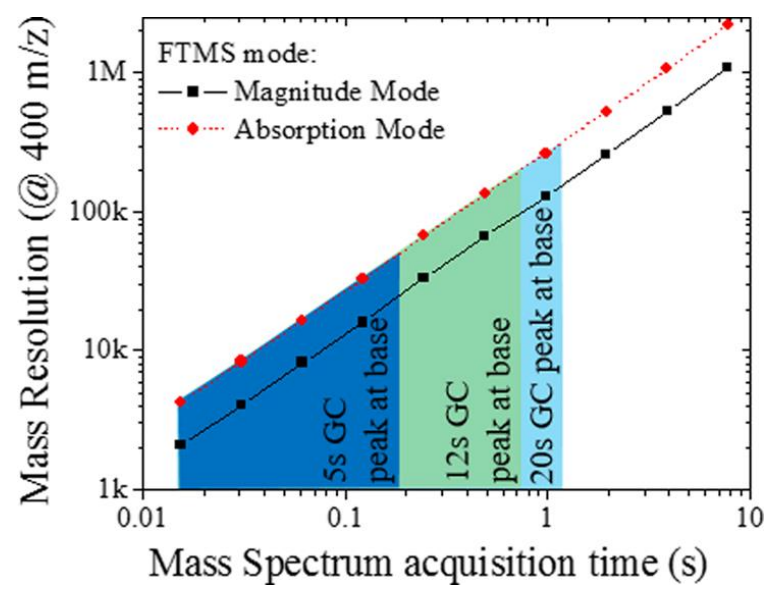

Figure 2.6. Typical mass resolution as a function of the transient time in a FT-ICR MS with an infinity cell (7.0 T magnet) using the magnitude and absorption modes. Notice that high mass resolution can be achieved for short GC peaks ( $5 \mathrm{~s}$ ) and ultrahigh mass resolution can be achieved for GC peaks in the range of 12-20 $\mathrm{s}$, assuming 15 FTICR-MS acquisitions per GC peak.

The GC-APLI-FT-ICR MS results shown are based on a GC method optimized for broad range elution and sensitivity. Overall, the GC-APLI-FT-ICR MS analytical power is 
determined by the rate and number of MS spectra that can be collected per GC peak in response to the analytical question see (Figure 2.6). Traditional GC-MS analysis of PAHs yields broad peaks with good analytical separation (e.g., typically $12 \mathrm{~s}^{72,73}$ and up to $20 \mathrm{~s}^{74}$ ). Assuming that 15 points are acquired across a GC peak, a 12 s GC signal translates to a FTMS transient of $0.8 \mathrm{~s}$, corresponding to $\sim 212,000$ mass resolution at $\mathrm{m} / z$ 400. In the case of shorter GC signals ( $5 \mathrm{~s}$ ), the GCAPLI-FT-ICR MS coupling can easily provide higher MS resolution (>85,000 at $\mathrm{m} / \mathrm{z}$ 400) than traditional MS analyzers (e.g., quadrupole, ion traps, and time-of-flight (TOF) analyzer). Our results showed that, while the biggest challenge in the GCFT-ICR MS coupling is the balance between the elution time window of a single compound and the acquisition time for sensitive and accurate mass detection, there are unique advantages in the use of this technique for complex mixtures, such as fossil oils.

\subsection{Conclusions}

The study of fossil oils requires innovative approaches to tackle the chemical diversity and complexity of these samples. In the present study, a GC-APLI source was successfully coupled to a FT-ICR MS for the analysis of fossil oils for the case of organics in shale oil (SRM 1580), petroleum crude oil (SRM 1582), heavy sweet crude oil (SRM 2722) reference standards. Results showed that multiple PAH classes can be easily identified with reduced number of contaminants and interferences when compared with other ionization sources. In particular, the addition of gas chromatography, prior to APLIFT-ICR MS, increases the ionization efficiency and signal-to-noise ratio of lower abundance fractions. In the mass range covered by the GC analysis $(m / z, 100-450)$, $13 \%-25 \%$ of the chemical identifications are unique to this method. In addition, it was 
shown that, by adequately balancing the GC separation and the FT-ICR MS acquisition transient, the GC-APLI-FT-ICR MS analysis provides over a 2-fold increase in the number of compounds detected when compared to APLI-FT-ICR MS. That is, the use of GC combined with APLI-FT-ICR MS in the analysis of PAHs results in higher molecular coverage, higher sensitivity, and the possibility to separate and identify molecular isomers from within a crude oil sample.

2.6 References

1.Rodgers, R. P.; McKenna, A. M. Anal. Chem. 2011, 83, 4665- 4687.

2.Marshall, A. G.; Rodgers, R. P. Proc. Natl. Acad. Sci. U. S. A. 2008, 105, 18090-18095.

3.Hegazi, A. H.; Andersson, J. T. Energy Fuels 2007, 21, 3375-3384.

4.Zadro, S.; Haken, J. K.; Pinczewski, W. V. J. Chromatogr. 1985, 323, 305-322.

5.Wang, F. C.-Y.; Qian, K.; Green, L. A. Anal. Chem. 2005, 77, 2777-2785.

6.Robbins, W. K. J. Chromatogr. Sci. 1998, 36, 457.

7.Kaminski, M.; Kartanowicz, R.; Gilgenast, E.; Namiesnik, J. Crit. Rev. Anal. Chem. 2005, 35, 193.

8.Fernandez-Lima, F. A.; Becker, C.; McKenna, A. M.; Rodgers, R.P.; Marshall, A. G.; Russell, D. H. Anal. Chem. 2009, 81, 9941-9947.

9.Ahmed, A.; Cho, Y.; Giles, K.; Riches, E.; Lee, J. W.; Kim, H. I.; Choi, C. H.; Kim, S. Anal. Chem. 2014, 86, 3300-3307.

10. Benigni, P.; Marin, R.; Fernandez-Lima, F. Int. J. Ion Mobility Spectrom. 2015, $18,151-157$.

11. Lalli, P. M.; Corilo, Y. E.; Rowland, S. M.; Marshall, A. G.; Rodgers, R. P. Energy Fuels 2015, 29, 3626-3633.

12. Robinson, E. W.; Garcia, D. E.; Leib, R. D.; Williams, E. R. Anal. Chem. 2006, 78, 2190-2198. 
13. Tang, X.; Bruce, J. E.; Hill, H. H. Rapid Commun. Mass Spectrom. 2007, 21, $1115-1122$.

14. Cho, Y.; Ahmed, A.; Islam, A.; Kim, S. Mass Spectrom. Rev. 2015, 34, 248.

15. Barrow, M. P.; Peru, K. M.; Headley, J. V. Anal. Chem. 2014, 86, 8281-8288.

16. Heffner, C.; Silwal, I.; Peckenham, J. M.; Solouki, T. Environ. Sci. Technol. 2007, 41, 5419-5425.

17. Luo, Z.; Heffner, C.; Solouki, T. J. Chromatogr. Sci. 2009, 47, 75-82. 266-271.

18. Hegazi, A. H.; Fathalla, E. M.; Andersson, J. T. Chemosphere 2014, 111,

19. Zhu, X.; Shi, Q.; Zhang, Y.; Pan, N.; Xu, C.; Chung, K. H.; Zhao, S. Energy Fuels 2011, 25, 281.

20. Headley, J. V.; Peru, K. M.; Mohamed, M. H.; Wilson, L.; McMartin, D. W.; Mapolelo, M. M.; Lobodin, V. V.; Rodgers, R. P.; Marshall, A. G. Energy Fuels 2014, 28, 1611-1616.

21. Bae, E.; Na, J.-G.; Chung, S. H.; Kim, H. S.; Kim, S. Energy Fuels 2010, 24, 2563-2569.

22. Rudzinski, W. E.; Oehlers, L.; Zhang, Y.; Najera, B. Energy Fuels 2002, 16, $1178-1185$.

23. Hsu, C. S.; Dechert, G. J.; Robbins, W. K.; Fukuda, E. K. Energy Fuels 2000, $14,217-223$.

24. Tose, L. V.; Cardoso, F. M.; Fleming, F. P.; Vicente, M. A.; Silva, S. R.; Aquije, G. M.; Vaz, B. G.; Romão, W. Fuel 2015, 153, 346-354.

25. Stader, C.; Beer, F. T.; Achten, C. Anal. Bioanal. Chem. 2013, 405, 7041-7052.

26. Schmitt-Kopplin, P.; Englmann, M.; Rossello-Mora, R.; Schiewek, R.; Brockmann, K. J.; Benter, T.; Schmitz, O. J. Anal. Bioanal. Chem. 2008, 391, 2803-2809.

27. Schiewek, R.; Schellenträger, M.; Mönnikes, R.; Lorenz, M.; Giese, R.; Brockmann, K.; Gäb, S.; Benter, T.; Schmitz, O. Anal. Chem. 2007, 79, 4135-4140.

28. Streibel, T.; Zimmermann, R. Annu. Rev. Anal. Chem. 2014, 7, 361-381.

29. Cho, Y.; Jin, J. M.; Witt, M.; Birdwell, J. E.; Na, J.-G.; Roh, N.-S.; Kim, S. Energy Fuels 2013, 27, 1830-1837. 
30. Terra, L. A.; Filgueiras, P. R.; Tose, L. V.; Romão, W.; de Castro, E. V.; de Oliveira, L. M.; Dias, J. C.; Vaz, B. G.; Poppi, R. J. Fuel 2015, 160, 274-281.

31. Pereira, T. M. C.; Vanini, G.; Tose, L. V.; Cardoso, F. M. R.; Fleming, F. P.; Rosa, P. T. V.; Thompson, C. J.; Castro, E. V. R.; Vaz, B. G.; Romão, W. Fuel 2014, 131, 49-58.

32. Rummel, J. L.; McKenna, A. M.; Marshall, A. G.; Eyler, J. R.; Powell, D. H. Rapid Commun. Mass Spectrom. 2010, 24, 784-790.

33. Romão, W.; Tose, L. V.; Vaz, B. G.; Sama, S. G.; Lobinski, R.; Giusti, P.; Carrier, H.; Bouyssiere, B. J. Am. Soc. Mass Spectrom. 2015, 1-4.

34. Wu, C.; Qian, K.; Nefliu, M.; Cooks, R. G. J. Am. Soc. Mass Spectrom. 2010, 21, 261-267.

35. Crawford, K. E.; Campbell, J. L.; Fiddler, M. N.; Duan, P.; Qian, K.; Gorbaty, M. L.; Kenttämaa, H. I. Anal. Chem. 2005, 77, 7916-7923.

36. Nyadong, L.; McKenna, A. M.; Hendrickson, C. L.; Rodgers, R.P.; Marshall, A. G. Anal. Chem. 2011, 83, 1616-1623.

37. Benassi, M.; Berisha, A.; Romão, W.; Babayev, E.; Römpp, A.; Spengler, B. Rapid Commun. Mass Spectrom. 2013, 27, 825-834.

38. Hsu, C. S.; Green, M. Rapid Commun. Mass Spectrom. 2001, 15, 236-239.

39. Peterson, A. C.; McAlister, G. C.; Quarmby, S. T.; Griep-Raming, J.; Coon, J. J. Anal. Chem. 2010, 82, 8618-8628.

40. Ledford, E. B.; White, R. L.; Ghaderi, S.; Wilkins, C. L.; Gross, M. L. Anal. Chem. 1980, 52, 2450-2451.

41. Szulejko, J.; Solouki, T. Anal. Chem. 2002, 74, 3434-3442.

42. Smit, E.; Rüger, C. P.; Sklorz, M.; De Goede, S.; Zimmermann, R.; Rohwer, E. R. Energy Fuels 2015, 29, 5554.

43. Lababidi, S.; Panda, S. K.; Andersson, J. T.; Schrader, W. Anal. Chem. 2013, $85,9478-9485$.

44. Lababidi, S.; Schrader, W. Rapid Commun. Mass Spectrom. 2014, 28, $1345-1352$.

45. Zimmermann, R.; Boesl, U.; Heger, H. J.; Rohwer, E. R.; Orthner, E. K.; Schlag, E. W.; Kettrup, A. J. J. High Resolut. Chromatogr. 1997, 20, 461-470. 
46. Mühlberger, F.; Zimmermann, R.; Kettrup, A. Anal. Chem. 2001, 73, 3590-3604.

47. Li, D.-X.; Gan, L.; Bronja, A.; Schmitz, O. J. Anal. Chim. Acta 2015, 891, $43-61$.

48. NIST Standard Reference Material 1580; National Institute of Science and Technology (NIST): Gaithersburg, MD, 2012.

49. NIST Standard Reference Material 1582; National Institute of Science and Technology (NIST): Gaithersburg, MD, 2012.

50. NIST Standard Reference Material 2722; National Institute of Science and Technology (NIST): Gaithersburg, MD, 2014.

51. Panda, S. K.; Brockmann, K.-J.; Benter, T.; Schrader, W. Rapid Commun. Mass Spectrom. 2011, 25, 2317-2326.

52. Qi, Y.; Barrow, M. P.; Li, H.; Meier, J. E.; Van Orden, S. L.; Thompson, C. J.; O’Connor, P. B. Anal. Chem. 2012, 84, 2923-2929.

53. Cho, Y.; Qi, Y.; O’Connor, P.; Barrow, M.; Kim, S. J. Am. Soc. Mass Spectrom. 2014, 25, 154-157.

54. Eilers, P. H. C. Anal. Chem. 2003, 75, 3631-3636.

55. Gaspar, A.; Zellermann, E.; Lababidi, S.; Reece, J.; Schrader, W. Anal. Chem. 2012, 84, 5257-5267.

56. Purcell, J. M.; Hendrickson, C. L.; Rodgers, R. P.; Marshall, A. G. Anal. Chem. 2006, 78, 5906-5912.

57. Fu, J.; Kim, S.; Rodgers, R. P.; Hendrickson, C. L.; Marshall, A. G.; Qian, K. Energy Fuels 2006, 20, 661-667.

58. Stanford, L. A.; Rodgers, R. P.; Marshall, A. G.; Czarnecki, J.; Wu, X. A.; Taylor, S. Energy Fuels 2007, 21, 973-981.

59. Kekäläinen, T.; Pakarinen, J. M. H.; Wickström, K.; Vainiotalo, P. Energy Fuels 2009, 23, 6055-6061.

60. McKenna, A. M.; Purcell, J. M.; Rodgers, R. P.; Marshall, A. G. Energy Fuels 2010, 24, 2929-2938.

61. Hur, M.; Yeo, I.; Kim, E.; No, M.-h.; Koh, J.; Cho, Y. J.; Lee, J. W.; Kim, S. Energy Fuels 2010, 24, 5524-5532. 
62. Gaspar, A.; Zellermann, E.; Lababidi, S.; Reece, J.; Schrader, W. Energy Fuels 2012, 26, 3481-3487.

63. Hsu, C. S.; Lobodin, V. V.; Rodgers, R. P.; McKenna, A. M.; Marshall, A. G. Energy Fuels 2011, 25, 2174-2178.

64. Ingram, L.; Ellis, J.; Crisp, P.; Cook, A. Chem. Geol. 1983, 38, 185-212.

65. Li, M.; Larter, S. R.; Stoddart, D.; Bjoroey, M. Anal. Chem. 1992, 64, $1337-1344$.

66. Hillier, J. L.; Fletcher, T. H.; Solum, M. S.; Pugmire, R. J. Ind. Eng. Chem. Res. 2013, 52, 15522-15532.

67. Schiewek, R.; Lorenz, M.; Giese, R.; Brockmann, K.; Benter, T.;Gäb, S.; Schmitz, O. J. Anal. Bioanal. Chem. 2008, 392, 87-96.

68. Rodgers, R. P.; Marshall, A. G. In Asphaltenes, Heavy Oils, and Petroleomics; Springer: Berlin, Heidelberg, Germany, 2007; pp 63-93.

69. Smith, D. F.; Rahimi, P.; Teclemariam, A.; Rodgers, R. P.; Marshall, A. G. Energy Fuels 2008, 22, 3118-3125.

70. Lorenz, M.; Schiewek, R.; Brockmann, K. J.; Schmitz, O. J.; Gäb, S.; Benter, T. J. Am. Soc. Mass Spectrom. 2008, 19, 400-410.

71. Harkewicz, R.; Belov, M.; Anderson, G.; Paša-Tolić, L.; Masselon, C.; Prior, D.; Udseth, H.; Smith, R. J. Am. Soc. Mass Spectrom. 2002, 13, 144-154.

72. Wise, S.; Poster, D.; Kucklick, J.; Keller, J.; VanderPol, S.; Sander, L.; Schantz, M. Anal. Bioanal. Chem. 2006, 386, 1153-1190.

73. Wise, S. A.; Schantz, M. M.; Benner, B. A., Jr.; Hays, M. J.; Schiller, S. B. Anal. Chem. 1995, 67, 1171-1178.

74. Wise, S.; Poster, D.; Leigh, S.; Rimmer, C.; Mössner, S.; Schubert, P.; Sander, L.; Schantz, M. Anal. Bioanal. Chem. 2010, 398, 717-728. 


\section{CHAPTER III}

FAST SCREENING OF POLYCYCLIC AROMATIC HYDROCARBONS USING TRAPPED ION MOBILITY SPECTROMETRY - MASS SPECTROMETRY

(Adapted from Castellanos, A., Benigni, P., et al., 2014, Analytical Methods, by permission of The Royal Society of Chemistry) 


\subsection{Abstract}

In the present paper, we showed the advantages of trapped ion mobility spectrometry coupled to mass spectrometry (TIMS-MS) combined with theoretical calculations for fast identification (millisecond timescale) of polycyclic aromatic hydrocarbons (PAH) compounds from complex mixtures. Accurate PAH collision cross sections (CCS, in nitrogen as a bath gas) are reported for the most commonly encountered $\mathrm{PAH}$ compounds and the ability to separate PAH geometric isomers is shown for three isobaric pairs with mobility resolution exceeding 150 (3-5 times higher than conventional IMS devices). Theoretical candidate structures (optimized at the DFT/B3LYP level) are proposed for the most commonly encountered PAH compounds showing good agreement with the experimental CCS value $(<5 \%)$. The potential of TIMS-MS for the separation and identification of PAH compounds from complex mixtures without the need of lengthy preseparation steps is illustrated for the case of a complex soil mixture.

\subsection{Introduction}

Over the last decade, there has been an increase in the demand for faster and more comprehensive analytical tools for the characterization and separation of PAHs from complex mixtures (see more details in ref. 1 and 2). The main challenges encountered during PAH characterization are attributable to the large number of compounds present in complex mixtures and their structural diversity. PAH incorporation into the environment occurs from multiple sources (e.g., oil spills, incomplete combustion processes, etc.) and one way to determine the origin of PAHs is by analyzing their molecular fingerprint. PAHs can also be incorporated into the human body via absorption in the gastrointestinal tract after ingestion of contaminated food or water and via skin contact. ${ }^{3-5}$ 
Despite recent efforts to improve the sensitivity and analytical specificity of PAHs quantification (e.g., SPE, ${ }^{6}$ SPME, ${ }^{7}$ LLE coupled to HPLC and GC-MS,${ }^{8}$ and GCxGCFID ${ }^{9}$ ), the development of more accurate analytical separations (e.g., post-ionization, gasphase separation) for molecular structure assignment with minimal to no sample preparation remains necessary.

Previous work has shown the advantage of ion mobility spectrometry coupled to mass spectrometry for the separation and identification of PAHs from crude oils with varying complexity. ${ }^{10}$ With the advent of trapped ion mobility spectrometry, higher analytical separation power and improved molecular characterization has become possible. ${ }^{11-13}$

In the present paper, we explore for the first time the capabilities of trapped ion mobility spectrometry coupled to mass spectrometry (TIMS-MS) combined with theoretical calculations for a faster and better characterization of PAH from complex mixtures. Accurate ion-neutral collision cross sections are measured for commonly encountered PAH standards and compared with candidate structures. The capability to separate (in the ms scale) and identify PAHs (by CCS and $\mathrm{m} / \mathrm{z}$ ) from a complex soil mixture is demonstrated.

\subsection{Experimental Section}

\subsubsection{Sample Preparation}

Individual standards of 2,3-benzanthracene, 1,2-benzanthracene, triphenylene, rubrene, pentacene, 1,2:5,6-dibenzanthracene, and 1,2,3,4-dibenzanthracene were purchased from Fisher Scientific (Waltham, MA). Pyrene, benzo(a)pyrene, benzo(e)pyrene, and perylene were obtained from Sigma Aldrich (St. Louis, MO). 
Chrysene was obtained from Ultra Scientific (North Kingstown, RI). All chemicals were used as received without further purification. Individual standards were diluted to a final concentration of $0.7 \mathrm{ng} \mathrm{ml}^{-1}$ in toluene (Fisher Scientific, Waltham, MA). The PAHs in soil certified research material which contains 16 PAHs at concentrations of $100 \mathrm{ppb}$ to $1 \mathrm{ppm}$ was obtained from Research Technologies Corporation (SQC017, Laramie, WY, details in appendix 3.1). The PAH containing soil was processed using ultrasonic extraction according to EPA method 3550C. A Tuning Mix mass spectrometry standard [Tunemix, G2421A, Agilent Technologies, Santa Clara, CAl was used as a mass and mobility calibration standard. Details on the Tuning Mix structures (e.g., $m / z 322 \mathrm{Ko}=1.376 \mathrm{~cm}^{2} \mathrm{~V}^{-}$ ${ }^{1} \mathrm{~s}^{-1}, m / z 622 \mathrm{Ko}=1.013 \mathrm{~cm}^{2} \mathrm{~V}^{-1} \mathrm{~s}^{-1}$, and $\left.\mathrm{m} / z 922 \mathrm{Ko}=0.835 \mathrm{~cm}^{2} \mathrm{~V}^{-1} \mathrm{~s}^{-1}\right)$ and TIMS mobility calibration procedures can be found elsewhere. ${ }^{13-14}$

\subsubsection{Sample Characterization}

Individual standards were analyzed using high resolution mass spectrometry to confirm the purity of the samples. High resolution mass spectrometry analysis was performed in a Solarix 7T FTICR-MS from Bruker Daltonics Inc. (Billerica, MA). An atmospheric pressure photo ionization source (APPI, based on the Apollo II design, Bruker Daltonics Inc., MA) using a Kr lamp with main emission bands at 10.0 and $10.6 \mathrm{eVwas}$ used for all analyses. All standards were observed with sub ppm mass accuracy.

\subsubsection{Fast separation by TIMS-MS}

Details regarding the TIMS operation and specifics compared to traditional IMS

can be found elsewhere. ${ }^{11-13}$ Briefly, in TIMS mobility separation is based on holding the ions stationary using an electric field against a moving gas. The separation in a TIMS 
device can be described by the center of the mass frame using the same principles as in a conventional IMS drift tube. ${ }^{15}$ Since mobility separation is related to the number of ion neutral collisions (or drift time in traditional drift tube cells), the mobility separation in a TIMS device depends on the bath gas drift velocity, ion confinement and ion elution parameters. The mobility, $\mathrm{K}$, of an ion in a TIMS cell is described by:

$$
K=\frac{v_{g}}{E}=\frac{A}{V_{\text {elution }}-V_{\text {base }}}
$$

where $\mathrm{vg}_{\mathrm{g}}, E, \mathrm{~V}_{\text {elution }}$ and $\mathrm{V}_{\text {base }}$ are the velocity of the gas, applied electric field, elution and base voltages, respectively. The constant $A$ can be determined using calibration standards of known mobilities. In TIMS operation, multiple geometric isomers/conformers are trapped simultaneously at different $E$ values resulting from a voltage gradient applied across the IMS tunnel. After thermalization, geometrical isomers/conformers are eluted by decreasing the electric field in stepwise decrements (referred to as the "ramp"). Each isomer/conformer eluting from the TIMS cell can be described by a characteristic voltage (i.e., $\mathrm{V}_{\text {elution }}-\mathrm{V}_{\text {base}}$ ). Eluted ions are then mass analyzed and detected by a maXis impact Q-ToF mass spectrometer (Bruker Daltonics Inc, Billerica, MA).

In a TIMS device, the total analysis time can be described as:

$$
\begin{gathered}
\text { Total IMS time }=T_{\text {trap }}+\left(\frac{V_{\text {elution }}}{V_{\text {ramp }}}\right) x T_{\text {ramp }}+\text { ToF } \\
=T_{0}+\left(\frac{V_{\text {elution }}}{V_{\text {ramp }}}\right) x T_{\text {ramp }}
\end{gathered}
$$


where, $\mathrm{T}_{\text {trap }}$ is the thermalization/trapping time, ToF is the time after the mobility separation, and Vramp and $T_{\text {ramp }}$ are the voltage range and time required to vary the electric field, respectively. The elution voltage can be experimentally determined by varying the ramp time for a constant ramp voltage. This procedure also determines the time ions spend outside the separation region To (e.g., ion trapping and time-of-flight).

The TIMS funnel is controlled using in-house software, written in National Instruments Lab VIEW, and synchronized with the maXis Impact Q-ToF acquisition program." Separation was performed using nitrogen as a bath gas at $c a .300 \mathrm{~K}$ and typical $P_{1}$ and $P_{2}$ values are 2.6 and 1.0 mbar, respectively. The same $R F(2040 \mathrm{kHz}$ and 200-350 Vpp) was applied to all electrodes including the entrance funnel, the mobility separating section, and the exit funnel.

Mobility values $(K)$ were correlated with $\operatorname{CCS}(\Omega)$ using the equation:

$$
\Omega=\frac{(18 \pi)^{1 / 2}}{16} \frac{z}{\left(k_{B} T\right)^{1 / 2}}\left[\frac{1}{m_{I}}+\frac{1}{m_{b}}\right]^{1 / 2} \frac{1}{K} \frac{760}{P} \frac{T}{273.15} \frac{1}{N^{*}}
$$

where $z$ is the charge of the ion, $\mathrm{k}_{\mathrm{B}}$ is the Boltzmann constant, $N^{*}$ is the number density at standard temperature and pressure and $m_{I}$ and $m_{b}$ refer to the masses of the ion and bath gas, respectively. ${ }^{15}$

\subsubsection{Theoretical Calculations}

Candidate structures were optimized at the DFT/B3LYP level with 6-31+g(d), 6$31++g(d, p)$, cc-pVDZ, and cc-pVTZ basis sets using Gaussian 09 software. ${ }^{16}$ Theoretical ion-neutral collision cross sections were calculated using MOBCAL version for helium ${ }^{17,18}$ 
and nitrogen ${ }^{19}$ as a bath gas at ca. $300 \mathrm{~K}$. Partial atomic charges were calculated using the Merz-Singh-Kollman scheme constrained to the molecular dipole moment. ${ }^{20}$ All optimized geometries and MOBCAL input files can be found in Appendix 3.3.
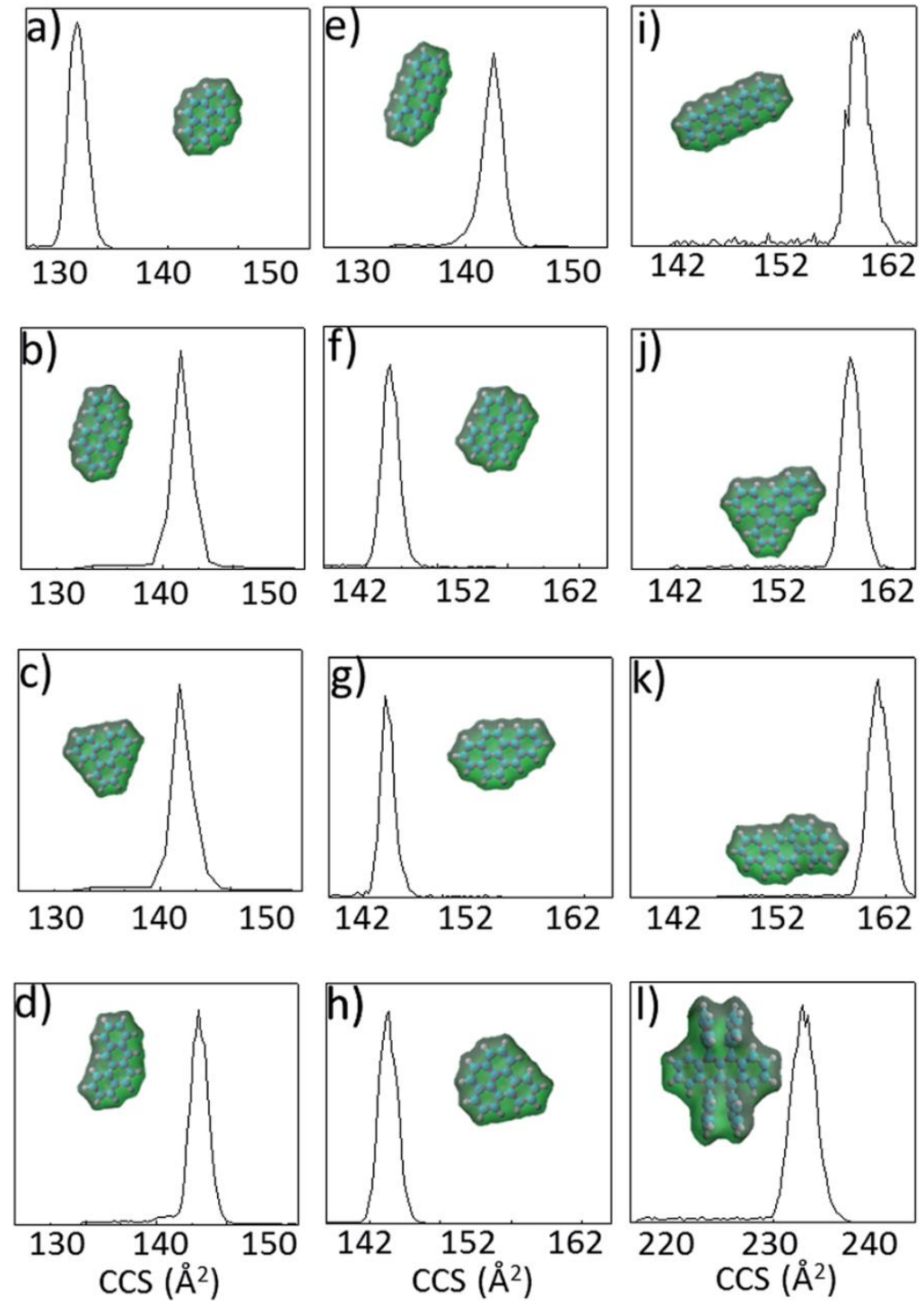

Figure 3.1 Typical TIMS spectra (Tramp $=500 \mathrm{~ms})$ of (a) pyrene $(\mathrm{R}=90)$, (b) chrysene $(\mathrm{R}=103)$, (c) triphenylene ( $\mathrm{R}=107)$, (d) 1,2-benzanthracene $(\mathrm{R}=98)$, (e) 2,3-benzanthracene $(\mathrm{R}=96)$, (f) perylene $(\mathrm{R}=$ 113), (g) benzo(a)pyrene $(\mathrm{R}=133)$, (h) benzo(e)pyrene $(\mathrm{R}=102)$, (i) pentacene $(\mathrm{R}=96)$, (j) 1,2,3,4dibenzanthracene $(\mathrm{R}=155)$, (k) 1,2:5,6-dibenzanthracene $(\mathrm{R}=98)$ and (l) rubrene $(\mathrm{R}=125)$. In the inset, the DFT/B3LYP/6-31++g $(\mathrm{d}, \mathrm{p})$ isosurface and a space-filled model representation of the molecular ions is shown.

\subsection{Results and Discussion}

Molecular ions $[\mathrm{M}]^{+}$for all the PAH standards were produce by APPI and introduced into the TIMS-MS instrument. The mass spectrometry signals of single PAHs 
were isolated $(\Delta m / z<1 \mathrm{Da}$, monoisotopic peak) and the corresponding TIMS spectra were collected (Fig. 3.1). As previously described in ref. 11, 13, 16 and 17, TIMS spectra were collected as a function of the ramp time for each isolated $m / z$ signal of the PAH $[\mathrm{M}]^{+}$ standards in order to calculate the elution voltage (see eqn (2)). Thereafter, mobility and ion-neutral (nitrogen) collision cross sections were calculated using Tuning Mix as external calibration (see Appendix 3.2). A good agreement is observed between the TIMS PAHs mobility and ion-nitrogen collision cross sections with previously reported values using drift-tube based IMS instruments. ${ }^{19,21}$

Candidate structures were proposed for all the PAH compounds (shown as inset of Fig. 3.1). Optimized geometries and partial atomic charges were calculated at the DFT/B3LYP level using multiple basis sets (see Appendix 3.2). With the increase of the

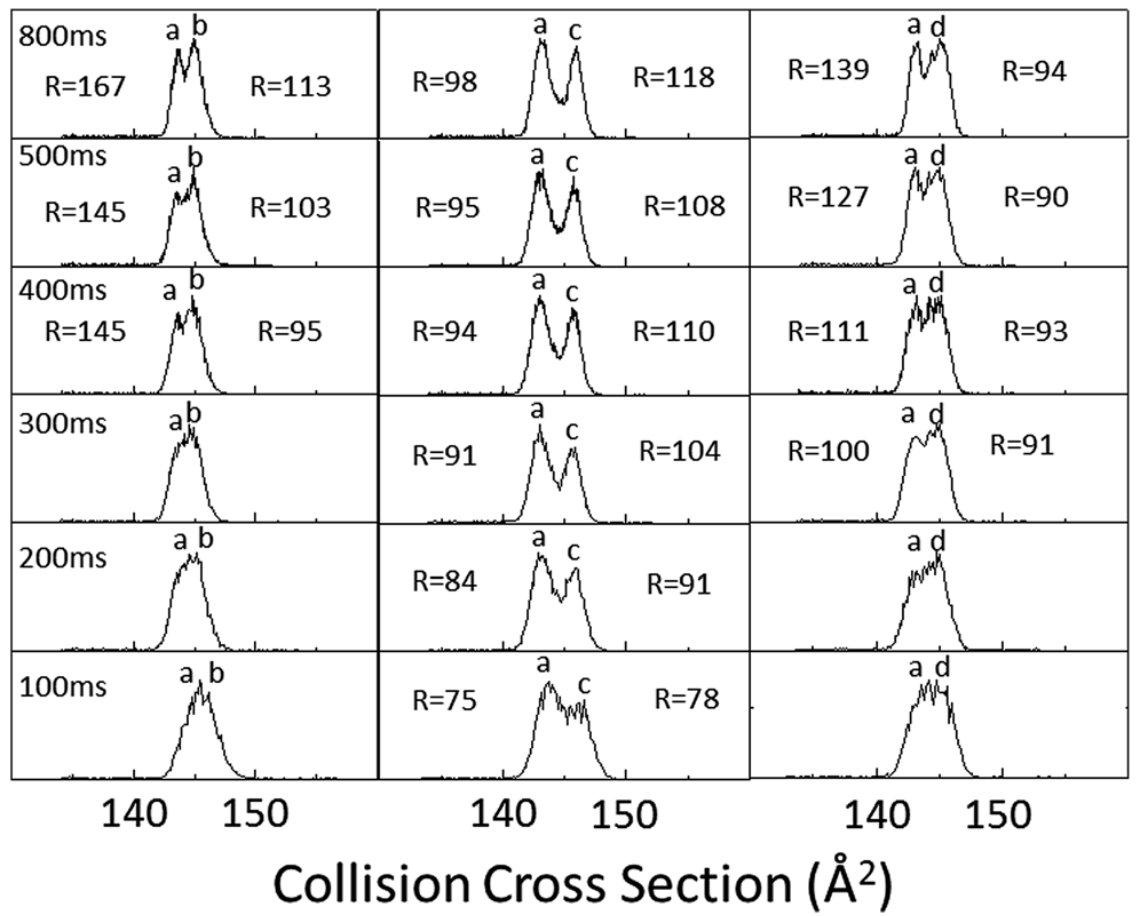

Figure 3.2 TIMS spectra as a function of the ramp time (100-800 ms) for two component isomeric mixtures of triphenylene (a), chrysene (b), 1,2-benzanthracene (c), and 2,3-benzanthracene (d). The TIMS peak resolution is shown for single components. 
basis sets, variations of less than $1 \%$ were observed in the $\mathrm{CCS}_{\mathrm{N}_{2}}$ for all the structures considered. A good agreement is observed between the theoretical and experimental ionneutral collision cross sections $(<5 \%)$.

A characteristic feature of TIMS is the ability to tune the mobility separation from low to high resolution by increasing the ramp time and narrowing the scan voltage across the ramp as a function of the analytical challenge. Typical mobility resolution of $\mathrm{R}=90$ 150 was obtained for a ramp time of $500 \mathrm{~ms}$ with a voltage ramp of a $\Delta \mathrm{V}=10 \mathrm{~V}$ for individual compounds.

It should be noted that TIMS analysis of PAH compounds comprises an additional challenge compared to previously studied biomolecules where higher TIMS mobility resolutions were obtained. ${ }^{12,13}$ That is, smaller $\mathrm{m} / z$ and CCS require the use of a lower electric field given the same bath velocity (resolution is directly related to the electric field strength). ${ }^{2,15}$ To better illustrate the TIMS capability to separate molecular PAH isobars $[\mathrm{M}]^{+}$, four isobaric pairs $\left(\mathrm{C}_{18} \mathrm{H}_{12}, \mathrm{~m} / z 228.093 \mathrm{Da}\right)$ : chrysene triphenylene, triphenylene

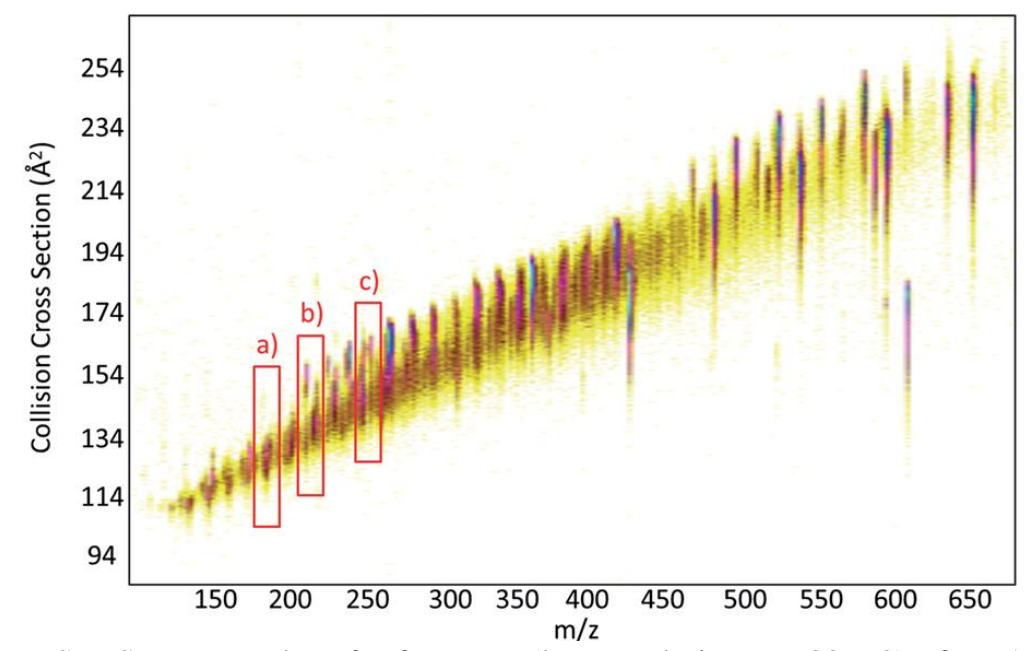

Figure 3.3. 2D TIMS-MS contour plot of a fast scan (low resolution $\mathrm{R}=30-60$ ) of a PAH mixture in soil (SC017) featuring over 16 different PAHs, including multiple sets of structural isomers. Regions $\mathrm{a}, \mathrm{b}$, and $\mathrm{c}$ correspond to $\mathrm{PAH}$ isomers with chemical formula $\mathrm{C}_{14} \mathrm{H}_{10}, \mathrm{C}_{18} \mathrm{H}_{12}$, and $\mathrm{C}_{20} \mathrm{H}_{12}$, respectively. 
vs. 1,2-benzathracene, and triphenylene vs. 2,3-benzathracene were studied as a function of the ramp time (Fig. 3.2). As the ramp time increases higher mobility separations are observed for all considered PAH isobaric pairs. Traditional PAH analysis of isobaric compounds involves the use of relatively slow separation methodologies (e.g., gas chromatography, GC or liquid chromatography, HPLC prior mass spectrometry analysis. Separation conditions are highly compound-specific, co-eluting PAH interferences must be specifically addressed and require operation times upwards of 40 minutes. ${ }^{9}$

The use of TIMS-MS for the separation of PAH-containing complex mixtures is illustrated for the case of a soil standard (SC017) featuring over 16 different PAHs, including sets of structural isomers (Fig. 3.3). Inspection of the 2D TIMS-MS contour plot shows a single trend line of singly charged species. Mobility projection plots are shown for three $m / z$ 's and illustrate the capability to separate molecular isobars in the TIMS domain (Fig. 3.4). Although multiple peaks are observed at the level of nominal mass, the mass resolution of the mass analyzer $(R=30-40 \mathrm{k})$ permitted the isolation of the PAH mass signal and the construction of the corresponding TIMS projection plot $(\mathrm{m} / z<0.2 \mathrm{Da}$, monoisotopic peak). Inspection of the mobility profiles shows that under theses low resolution TIMS conditions (70 ms ramp time of and $20 \mathrm{~V}_{\text {ramp }}$ ) some isobaric pairs are resolved (Fig. 3.4b and c) while other pairs are not resolved (Fig. 3.4a). Comparison with the theoretical and single standard values shows that peaks that are not resolved have similar mobility values in a nitrogen bath gas (e.g., anthracene and phenanthrene $\mathrm{CCS}_{\mathrm{N}_{2}}=$ $128 \AA^{2}$ ). Moreover, the PAH isobars that are resolved (Fig. 4b and c) showed the correct relative abundance as the certified concentrations specified for the sample (see Appendix 
3.1). The analysis time to generate a TIMS-MS data set is typically on the order of a few minutes, and low signal intensities from low abundance components can be compensated by summing multiple datasets. Additionally, TIMS can improve signal-to-noise ratio in cases where the peak width is less than the ion accumulation time similar to conventional chromatography.
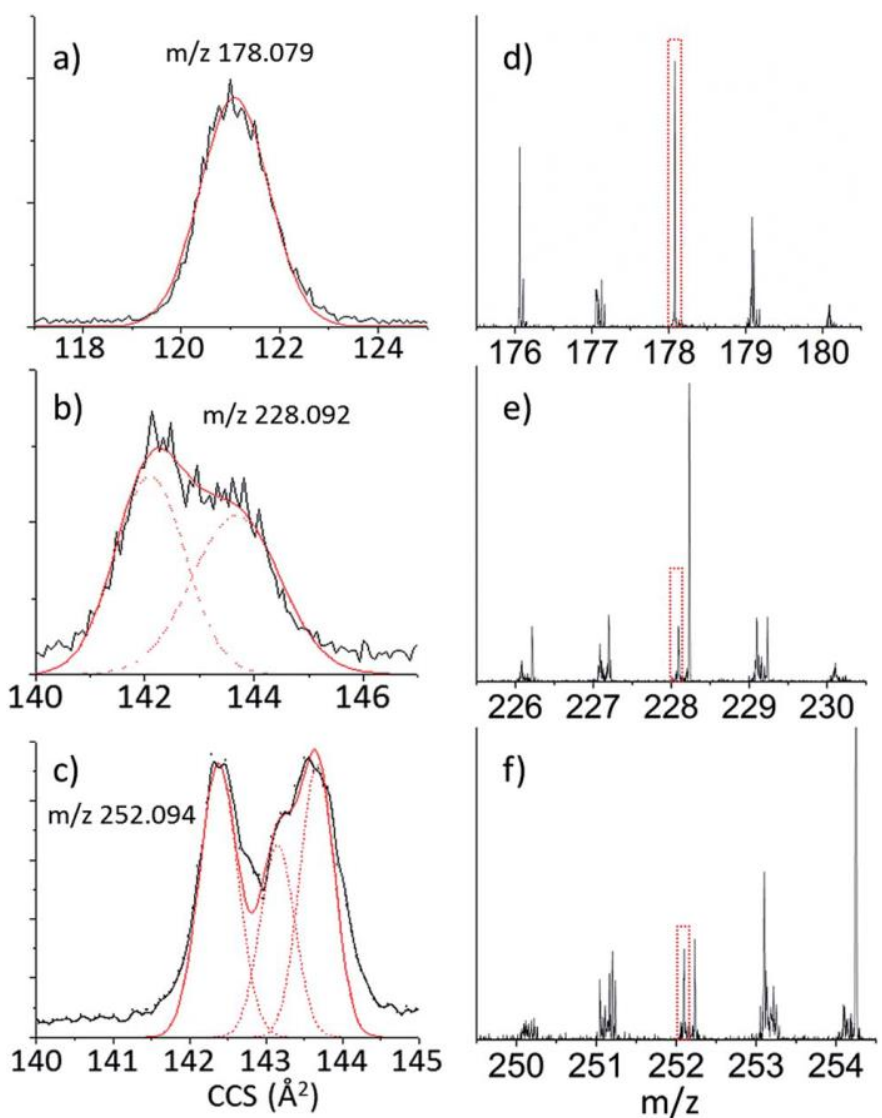

Figure 3.4 TIMS and MS projection plots for the insets shown in Fig. 3.3 of the structural isomers (a) phenanthracene and anthracene (not resolved), (b) chrysene (56\%) and 1,2-benzanthracene (44\%), and (c) benzo(a)pyrene (45\%), benzo(b)fluoranthene(14\%) and benzo(k)fluoranthene $(41 \%)$. (d-f) Excerpts of the mass spectrum corresponding to the compounds in $(\mathrm{a}-\mathrm{c})$.

\subsection{Conclusions}

The present study showed that comprehensive and fast characterization of PAH from complex mixture is feasible using TIMS-MS in the millisecond scale without the need of lengthy pre-fractionation separations. TIMS-MS has the added benefit that when 
combined with theoretical calculations permits the characterization of the conformational space of PAH and their separation from other chemical classes in a single analysis (or scan). Accurate ion-neutral collision cross sections were obtained for commonly encountered PAHs in the $m / z$ 124-523 with $\mathrm{CCS}_{\mathrm{N}_{2}}=114-232 \AA^{2}$ range. A good agreement was obtained between the theoretical and experimental ion-neutral collision cross sections $(<5 \%)$. The unique capability of TIMS to tune mobility resolution from low $(\mathrm{R}=30-60)$ to high $(\mathrm{R}=90-150)$ as a function of the analytical challenge was shown for the case of PAHs compounds from a soil extract.

\subsection{References}

1.B. R. T. Simoneit, Mass Spectrom. Rev., 2005, 24 , 719-765.

2.R. P. Rodgers and A. M. McKenna, Anal. Chem., 2011, 83, 4665-4687.

3.J. Angerer, C. Mannschreck and J. Gundel, Int. Arch. Occup. Environ. Health, 1997, 70, 365; B. Marczynski, R. Preuss, T. Mensing, J. Angerer, A. Seidel, A. El Mourabit, M. Wilhelm and T. Bruning, Int. Arch. Occup. Environ. Health, 2005, 78, 97.

4.J. Jacob and A. Seidel, J. Chromatogr. B, 2002, 778 , 31.

5.F. J. Jongeneelen, Sci. Total Environ., 1997, 199, 141.

6.B. Rossbach, R. Preuss, S. Letzel, H. Drexler and J. Angerer, Int. Arch. Occup. Environ. Health, 2007, 81, 221.

7.C. J. Smith, C. J. Walcott, W. Huang, V. Maggio, J. Grainger and D. G. Patterson Jr, J. Chromatogr. B , 2002, 778, 157.

8.N. Grova, F. Monteau, B. Le Bizec, C. Feidt, F. Andre and G. Rychen, J. Anal. Toxicol., 2005, 29, 175; Z. Li, L. C. Romanoff, D. A. Trinidad, N. Hussain, R. S. Jones, E. N. Porter, D. G. Patterson and A. Sjodin, Anal. Chem.,2006, 78, 5744; G. Gmeiner, P. Gartner, C. Krassnig and H. Tausch, J. Chromatogr. B, 2002, 766, 209.

9.L. C. A. Amorim, J. M. Dimandja and Z. L. Cardeal, J. Chromatogr. A, 2009, 1216, 2900-2904. 
10. F. A. Fernandez-Lima, C. Becker, A. M. McKenna, R. P. Rodgers, A. G. Marshall and D. H. Russell, Anal. Chem., 2009, 81, 9941-9947; A. Ahmed, Y. Cho, K. Giles, E. Riches, J. W. Lee, H. I. Kim, C. H. Choi and S. Kim, Anal. Chem., 2014, 86, 3300-3307; A. Ahmed, Y. J. Cho, M. H. No, J. Koh, N. Tomczyk, K. Giles, J. S. Yoo and S. Kim, Anal. Chem., 2010, 83, 77; F. Maire, K. Neeson, R. Denny, M. McCullagh, C. Lange, C. Afonso and P. Giusti, Anal. Chem., 2013, 85, 5530.

11. F. A. Fernandez-Lima, D. A. Kaplan and M. A. Park, Rev. Sci. Instrum., 2011, 82 , 126106; F. A. Fernandez-Lima, D. A. Kaplan, J. Suetering and M. A. Park, Int. J. Ion Mobility Spectrom., 2011, 14 ,93-98.

12. E. R. Schenk, M. E. Ridgeway, M. A. Park, F. Leng and F. Fernandez-Lima, Anal. Chem., 2013, 86, 1210-1214; E. R. Schenk, V. Mendez, J. T. Landrum, M. E. Ridgeway, M. A. Park and F. Fernandez-Lima, Anal. Chem., 2014, 86, 2019-2024.

13. D. R. Hernandez, J. D. DeBord, M. E. Ridgeway, D. A. Kaplan, M. A. Park and F. Fernandez-Lima, Analyst, 2014, 139, 1913-1921.

14. L. A. Flanagan, US Pat., 5872357, 1999.

15. E. W. McDaniel and E. A. Mason, Mobility and diffusion of ions in gases, John Wiley and Sons, Inc., New York, New York, 1973, p. 381.

16. M. J. Frisch, G. W. Trucks, H. B. Schlegel, G. E. Scuseria, M. A. Robb, J. R. Cheeseman, J. J. A. Montgomery, T. Vreven, K. N. Kudin, J. C. Burant, J. M. Millam, S. S. Iyengar, J. Tomasi, V. Barone, B. Mennucci, M. Cossi, G. Scalmani, N. Rega, G. A. Petersson, H. Nakatsuji, M. Hada, M. Ehara, K. Toyota, R. Fukuda, J. Hasegawa, M. Ishida, T. Nakajima, Y. Honda, O. Kitao, H. Nakai, M. Klene, X. Li, J. E. Knox, H. P. Hratchian, J. B. Cross, V. Bakken, C. Adamo, J. Jaramillo, R. Gomperts, R. E. Stratmann, O. Yazyev, A. J. Austin, R. Cammi, C. Pomelli, J. W. Ochterski, P. Y. Ayala, K. Morokuma, G. A. Voth, P. Salvador, J. J. Dannenberg, V. G. Zakrzewski, S. Dapprich, A. D. Daniels, M. C. Strain, O. Farkas, D. K. Malick, A. D. Rabuck, K. Raghavachari, J. B. Foresman, J. V. Ortiz, Q. Cui, A. G. Baboul, S. Clifford, J. Cioslowski, B. B. Stefanov, G. Liu, A. Liashenko, P. Piskorz, I. Komaromi, R. L. Martin, D. J. Fox, T. Keith, M. A. AlLaham, C. Y. Peng, A. Nanayakkara, M. Challacombe, P. M. W. Gill, B. Johnson, W. Chen, M. W. Wong, C. Gonzalez and J. A. Pople, in Gaussian, Wallingford CT, 2004.

17. M. F. Mesleh, J. M. Hunter, A. A. Shvartsburg, G. C. Schatz and M. F. Jarrold,J. Phys. Chem., 1996, 100, 16082-16086.

18. A. A. Shvartsburg and M. F. Jarrold, Chem. Phys. Lett., 1996,261,86-91.

19. I. Campuzano, M. F. Bush, C. V. Robinson, C. Beaumont, K. Richardson, H. Kim and H. I. Kim, Anal. Chem., 2011, 84, 1026-1033. 
20. U. C. Singh and P. A. J. Kollman, J. Comput. Chem., 1984, 5,129-145; B. H. Besler, K. M. Merz and P. A. Kollman,J. Comput. Chem., 1990,11, 431-439.

21. D. Young, K. M. Douglas, G. A. Eiceman, D. A. Lake and M. V. Johnston, Anal. Chim. Acta, 2002, 453, 231-243. 
CHAPTER IV

ANALYSIS OF GEOLOGICALLY RELEVANT METAL PORPHYRINS USING TRAPPED ION MOBILITY SPECTROMETRY-MASS SPECTROMETRY AND THEORETICAL CALCULATIONS

(Adapted with permission from Benigni et al., 2016, Energy and Fuels, copyright 2017 American Chemical Society) 


\subsection{Abstract}

The structural characterization of metal porphyrins has been traditionally challenging as a result of their large structural and compositional diversity. In the present paper, we show the advantages of gas-phase, postionization separations for the fast identification and structural characterization of metal octaethylporphyrins (Me-OEP) from complex mixtures using trapped ion mobility spectrometry (TIMS) coupled to ultrahighresolution mass spectrometry (FT-ICR MS). TIMS-FT-ICR MS allows for the separation of Me-OEP $(\mathrm{Me}=\mathrm{Mn}, \mathrm{Ni}, \mathrm{Zn}, \mathrm{V}=\mathrm{O}$, and $\mathrm{Ti}=\mathrm{O})$ within a crude oil sample based on accurate mass and mobility signatures (with a mobility resolving power of $\mathrm{R}_{\mathrm{IMS}} \sim 150$ 250). Accurate collision cross sections are reported for Me-OEP in nitrogen as bath gas $\left(\mathrm{CCS}_{\mathrm{N}_{2}}\right)$. Inspection of the Me-OEP mobility spectra showed a single mobility component distribution for $\mathrm{Me}-\mathrm{OEP}(\mathrm{Me}=\mathrm{Mn}, \mathrm{Ni}$, and $\mathrm{Zn})$ and a multi-component distribution for the two metal carbonyls, vanadyl $(\mathrm{V}=\mathrm{O})$ and titanyl $(\mathrm{Ti}=\mathrm{O}) \mathrm{Me}-\mathrm{OEP}$. Candidate structures were proposed at the DFT/B3LYP/6-31g(d) level for all Me-OEP mobility bands observed. Inspection of the optimized Me-OEP candidate structures shows that manganese, zinc, and free OEP adopt a planar conformation, the nickel-complexed OEP structure adopts a "ruffled" conformation; and the metal oxide OEP adopts a dome conformation, with carbonyl pointing upward, perpendicular to the plane of the structure.

\subsection{Introduction}

In the early 1930s, Alfred Treibs isolated porphyrins from a range of geological materials, including crude oils, oil shales, coals, and phosphorites. ${ }^{1-4}$ The discovery provided strong evidence for the biological origin of crude oil, as well as indicating that crude oil was generated at temperatures below $300{ }^{\circ} \mathrm{C}$. The work by Treibs culminated in 
the postulation of the Treibs hypothesis that biological molecules underwent a series of transformations in which functional groups were either lost or converted to the appropriate alkyl moieties, as shown in Scheme 4.1. This hypothesis laid the foundation of organic geochemistry at the molecular level. ${ }^{5}$ Subsequently, geoporphyrins have proven to be valuable in oil exploration, where the ratio of nickel/vanadium porphyrins and the ratio of deoxophylloerythroetioporphyrin (DPEP)/etioporphyrin are used as maturation parameters. ${ }^{6}$ In addition, the distribution of porphyrins in oils and shales can be used for correlation of crude oils with other crude oils and crude oils with potential source rocks. ${ }^{7,8}$

Scheme 4.1. Diagram Illustrating the Chemical Changes Outlined in the Treibs Hypothesis ${ }^{\mathrm{a}}$

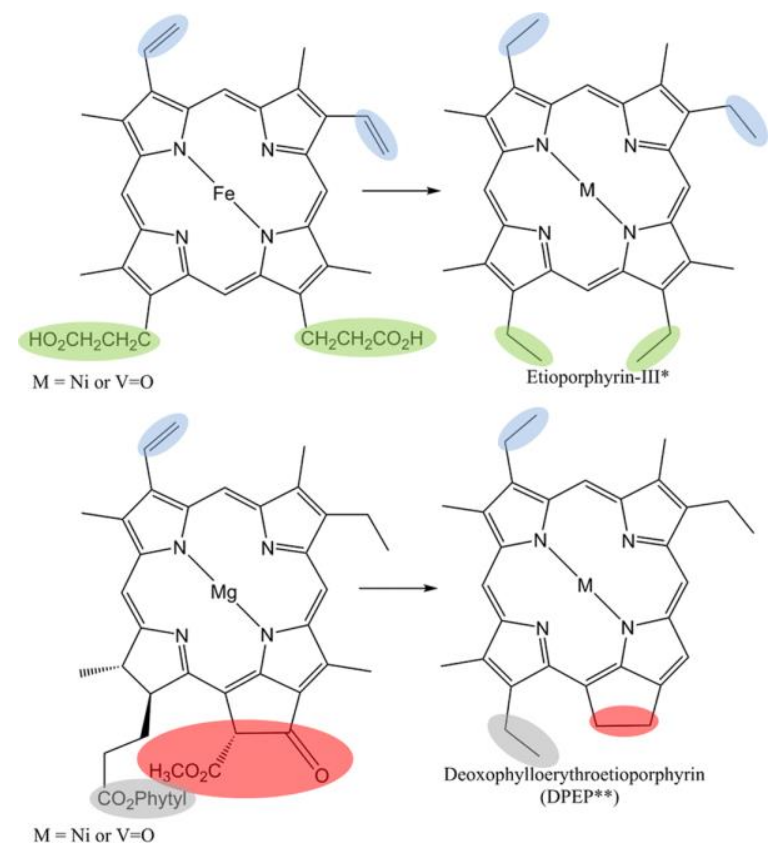

${ }^{a}$ Note the changes in the different functional groups observed.

Geoporhyrins occur with a wide variety of skeletal types, of which DPEP and etioporphyrin (Scheme 4.1) are the most important. They are typically found as either the $\operatorname{vanadyl}(\mathrm{V}=\mathrm{O})$ or nickel complexes. Metal-free geoporphyrins do exist in nature but are rare. ${ }^{9}$ Likewise, there have been reports of gallium, iron, and manganese porphyrins in 
coal $^{10}$ and copper porphyrins in shale. ${ }^{11}$ The vanadyl and nickel porphyrins are the most prevalent metal complexes found in oil and shale $;{ }^{12}$ however, these have an unfortunate property of damaging cracking catalysts used in the refining process and are often called the "red peril" of the oil refinery.

Identification of geoporphyrins is traditionally challenging because of the wide variety of structures as well as the complexity of the matrix in which they are typically found. Traditionally, liquid chromatography has been used with X-ray crystallography, ultraviolet-visible (UV-vis) ${ }^{13}$ Fourier transform infrared spectroscopy (FTIR), nuclear magnetic resonance (NMR), ${ }^{14}$ and inductively coupled plasma mass spectrometry (ICPMS) ${ }^{15}$ for the characterization and quantification of porphyrins. ${ }^{16}$ In recent years, mass spectrometry (MS)-based technologies have been developed and used for the molecular characterization of crude oils. Particularly, Fourier transform ion cyclotron resonance mass spectrometry (FT-ICR MS) has been coupled with electrospray ionization (ESI), ${ }^{17}, 18$ atmospheric pressure photoionization (APPI), ${ }^{19-22}$ direct analysis in real time (DART) ${ }^{23,24}$ and laser desorption ionization (LDI) ${ }^{25,26}$ for the analysis of vanadyl and nickel porphyrins, without the use of pre-separation, as well as structural elucidation by tandem MS techniques using electron-induced dissociation of $\mathrm{Fe}(\mathrm{III})$ octaethylporphyrin ${ }^{27}$ and $405 \mathrm{~nm}$ photofragmentation of hemin ${ }^{+28}$ Although MS techniques are capable of identifying porphyrins by their chemical formula and some structural features by tandem MS, elucidation of the tridimensional structure and conformation has not been reported.

Molecular characterization of complex mixtures using ion mobility spectrometry coupled to MS (IMS-MS) is rapidly becoming the analytical gold standard that combines the power of ultrahigh-resolution MS with the isomeric separation and structural 
identification capabilities of IMS. ${ }^{29-35}$ Moreover, the IMS measurement, when complemented with theoretical calculations, has proven to be a powerful technique for structural molecular analysis by correlating the ion-neutral, collision cross sections (CCS) with candidate structures. ${ }^{36-43}$ Specifically, we have shown the potential of trapped ion mobility spectrometry (TIMS) for the analysis of small (e.g., polyaromatic hydrocarbons, ${ }^{44}$ explosives, ${ }^{45}$ and metabolites ${ }^{46}$ ) and large (e.g., peptides, protein, DNA, and their complexes ${ }^{47-50}$ ) molecules with IMS resolving powers of up to $400 .{ }^{46}$ The advantages of coupling TIMS to FT-ICR MS has been shown for the case of endocrine disruptors, ${ }^{51}$ glycans, ${ }^{52}$ structural and conformational peptide isomers,${ }^{53}$ and crude oils. ${ }^{53}$

In this study, we use, for the first time, the advantages of TIMS and FT-ICR MS for the separation and structural characterization of metal octaethylporphyrins (Me-OEP) in crude oils. This study provides the first tridimensional characterization of Me-OEP combining accurate collision cross section measurements in nitrogen with candidate structure generation at the DFT/B3LYP level for the characterization of Me-OEP conformational space.

\subsection{Experimental Section}

\subsubsection{Sample Preparation}

Octaethylporphyrin (OEP) samples were obtained from Sigma-Aldrich and used as received. Titanyl-OEP was prepared by heating OEP with $\mathrm{TiO}(\mathrm{acac})_{2}$ and phenol following the method by Buchler et al. ${ }^{54}$ Samples were dissolved in methylene chloride and subsequently diluted with methanol with $1 \%(\mathrm{v} / \mathrm{v})$ acetic acid. A standard reference material of polycyclic aromatic hydrocarbons (PAHs) in coal tar (SRM 1597a) was purchased from the National Institute of Standards and Technology (Gaithersburg, MD) 
and was diluted 1:100 in 50:50 (v/v) methanol/toluene. A tuning mix mass spectrometry standard (Tunemix, G2421A, Agilent Technologies, Santa Clara, CA) was used as a mobility calibration standard. Details on the Tunemix structures (e.g., $m / z, 322$, Ko $=1.376$ $\mathrm{cm} 2 \mathrm{~V}-1 \mathrm{~s}-1 ; m / z$ 622, Ko $=1.013 \mathrm{~cm} 2 \mathrm{~V}-1 \mathrm{~s}-1 ;$ and $m / z$ 922, Ko $=0.835 \mathrm{~cm} 2 \mathrm{~V}-1 \mathrm{~s}-1)$ can be found in ref 55. An APPI source (Apollo II, Bruker Daltonics, Inc., Billerica, MA) using a $\mathrm{Kr}$ lamp with main emission bands at 10.0 and $10.6 \mathrm{eV}$ was used for all of the analyses. Acetone was used as an APPI dopant (10\% by volume).

\subsubsection{Structural Separation by TIMS-MS}

Details regarding TIMS operation and differences from traditional IMS can be

found elsewhere. ${ }^{53,56,57}$ Ion mobility separation in the TIMS occurs when ions in the TIMS tunnel experience a drag force as a result of a moving gas of velocity $\mathrm{v}_{\mathrm{g}}$ and are compensated by an electric field (E), which holds the ions stationary within the tunnel. The mobility, $\mathrm{K}$, of an ion in a TIMS cell is described by

$$
K=\frac{v_{8}}{E}=\frac{A}{\left(V_{\text {elution }}-V_{\text {out }}\right)}
$$

where $\mathrm{V}_{\mathrm{g}}, \mathrm{E}, \mathrm{V}_{\text {elution}}$, and $\mathrm{V}_{\text {out }}$ are the velocity of the gas, applied electric field, elution voltage, and tunnel out voltage, respectively. The constant $\mathrm{A}$ is determined from calibration with known standards (e.g., Tunemix and G2421A).

Typical experimental parameters used nitrogen as a bath gas at ca. $300 \mathrm{~K}$, and typical P1 and P2 values are 2.6 and 1.0 mbar, respectively. The same radio frequency (RF, $880 \mathrm{kHz}$ and $200-350 \mathrm{Vpp}$ ) was applied to all electrodes, including the entrance funnel, 
the mobility separating section, and the exit funnel. Mobility values (K) were correlated with $\operatorname{CCS}(\Omega)$ using the equation

$$
\Omega=\frac{(18 \pi)^{1 / 2}}{16} \frac{z}{\left(k_{\mathrm{B}} T\right)^{1 / 2}}\left[\frac{1}{m_{\mathrm{l}}}+\frac{1}{m_{\mathrm{b}}}\right]^{1 / 2} \frac{1}{K} \frac{760}{P} \frac{T}{273.15} \frac{1}{N^{*}}
$$

where $\mathrm{z}$ is the charge of the ion, $\mathrm{k}_{\mathrm{B}}$ is the Boltzmann constant, $\mathrm{N}^{*}$ is the number density, and $m_{I}$ and $m_{b}$ refer to the masses of the ion and bath gas, respectively. ${ }^{58}$

\subsubsection{TIMS-FT-ICR MS Analysis}

An orthogonal, commercial APPI source based on the Apollo II design (Bruker Daltonics, Inc., Billerica, MA) with a Kr lamp with main emission bands at 10.0 and 10.6 eV was used. Briefly, sample solutions were introduced into the nebulizer at a rate of 300 $\mu \mathrm{L} / \mathrm{min}$ using an external syringe pump. Typical source operating conditions were a 2 $\mathrm{L} / \mathrm{min}$ dry gas flow rate, a 0.5 bar nebulizer gas pressure, a $220{ }^{\circ} \mathrm{C}$ dry gas temperature, and a $300{ }^{\circ} \mathrm{C}$ vaporizer temperature. Ions from the APPI source were introduced via a 0.6 mm inner diameter, single-bore glass capillary tube, which is resistively coated across its length, allowing the nebulizer to be maintained at ground potential, while the exit end of the capillary can be independently biased (typical values are -900 and $60-180 \mathrm{~V}$ for the entrance and exit, respectively).

TIMS separation used nitrogen as a bath gas at ca. $300 \mathrm{~K}$ with the gas flow velocity controlled by the pressure difference between the entrance funnel P1 $=2.6-3.0$ mbar and the exit funnel P2 = 1.5-1.6 mbar. TIMS voltage sequences were controlled using in-house software, written in National Instruments LabVIEW (version 12.0f3), and synchronized with the FT-ICR MS acquisition program. The TIMS cell was operated using a 
fill/trap/elute/quench sequence of 300/50/40/10 ms, collecting a single FT-ICR MS spectrum for each mobility trapping step. FT-ICR MS operation was optimized for ion transmission in the $m / z 150-1500$ range and processed using sine-squared windowing in magnitude mode. TIMS analysis was performed in oversampling mode ${ }^{53}$ with a $\Delta \mathrm{V}_{\text {ramp }}$ of $1 \mathrm{~V}$ in the range from -25 to $-180 \mathrm{~V}$ and a $\mathrm{V}_{\text {step }}$ of $0.2 \mathrm{~V}$; a total of 30 mobility steps were accumulated in the collision cell prior to injection in the ICR cell. FT-ICR MS and TIMSFT-ICR MS spectra were acquired at 8 Mword ( $6 \mathrm{~s}$ transient and $\mathrm{R}_{\mathrm{MS}}=600,000$ at $\mathrm{m} / \mathrm{z} 400$, using an average of 100 scans) and 512000 word ( $\mathrm{R}_{\mathrm{MS}}=35,000$ at $\mathrm{m} / \mathrm{z} 400$, using single scan), respectively. Details for data processing can be found elsewhere. ${ }^{53}$

\subsubsection{Theoretical Calculations}

A pool of Me-OEP candidate structures was obtained from previously reported crystal structures. ${ }^{59-66}$ Candidate structures were proposed on the basis of the stoichiometry of the Me-OEP ions observed during the TIMS-FT-ICR MS experiments. Me-OEP structures were protonated at the bridge, nitrogen, and oxygen, for the metal oxide structures, prior to geometry energy optimization and frequency calculation at the B3LYP/6-31g(d) level. ${ }^{67}$ Zero-point vibrational energy corrections obtained from the calculations of vibrational frequencies were included in all energies of Me-OEP. Partial atomic charges were calculated using the Merz-Singh-Kollman scheme constrained to the Table 4.1. Experimental Mass ( $\mathrm{m} / \mathrm{z}$ ), Mobility (Ko), and CCS for Me-OEP Studied Using TIMS-FT-ICR $\mathrm{MS}^{\mathrm{a}}$

\begin{tabular}{|c|c|c|c|c|c|c|}
\hline $\mathrm{Me}-\mathrm{OEP}$ & experimental mass $(\mathrm{m} / \mathrm{z})$ & theoretical mass $(\mathrm{m} / \mathrm{z})$ & formula & mobility, $\mathrm{K}_{0}\left(\mathrm{~cm}^{2} \mathrm{~s}^{-1} \mathrm{~V}^{-1}\right)$ & $\operatorname{CCS}\left(\AA^{2}\right)$ & theoretical CCS \\
\hline$[\mathrm{M}+\mathrm{H}]^{+}$ & 535.379 & 535.379 & {$\left[\mathrm{C}_{36} \mathrm{H}_{47} \mathrm{~N}_{4}\right]^{+}$} & 0.850 & 242 & 248 \\
\hline$[\mathrm{M}+\mathrm{Mn}-2 \mathrm{H}]^{+}$ & 587.294 & 587.294 & {$\left[\mathrm{C}_{36} \mathrm{H}_{44} \mathrm{~N}_{4} \mathrm{Mn}\right]^{+}$} & 0.844 & 244 & 248 \\
\hline$[\mathrm{M}+\mathrm{Ni}-\mathrm{H}]^{+}$ & 591.299 & 591.299 & {$\left[\mathrm{C}_{36} \mathrm{H}_{45} \mathrm{~N}_{4} \mathrm{Ni}\right]^{+}$} & 0.846 & 244 & 248 \\
\hline$[\mathrm{M}+\mathrm{Zn}-\mathrm{H}]^{+}$ & 597.293 & 597.293 & {$\left[\mathrm{C}_{36} \mathrm{H}_{45} \mathrm{~N}_{4} \mathrm{Zn}\right]^{+}$} & 0.839 & 245 & 247 \\
\hline \multirow[t]{2}{*}[\mathrm{M}+\mathrm{VO}-\mathrm{H}]{$^{+}$} & 600.302 & 600.303 & {$\left[\mathrm{C}_{36} \mathrm{H}_{45} \mathrm{~N}_{4} \mathrm{VO}\right]^{+}$} & 0.841 & 244 & 249 \\
\hline & & & & 0.833 & 246 & 248 \\
\hline$[\mathrm{M}+\mathrm{TiO}-\mathrm{H}]^{+}$ & 597.307 & 597.307 & {$\left[\mathrm{C}_{36} \mathrm{H}_{45} \mathrm{~N}_{4} \mathrm{TiO}\right]^{+}$} & 0.823 & 249 & 250 \\
\hline aTh & & 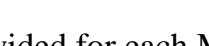 & & 0.832 & 247 & 249 \\
\hline
\end{tabular}

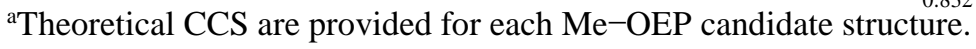


molecular dipole moment. ${ }^{68,69}$ Theoretical mobility values were calculated using the $70 \%$ diffuse hard sphere scattering (DHSS) model (3,000,000 nitrogen molecules per rotation, three rotations, 92\% Maxwell distributed remission velocity, and 70\% accommodation) in IMoS software (version 1.06W64d). ${ }^{70-72}$ Details on the optimized Me-OEP geometries can be found in Appendix 4.1 .

\subsection{Results and Discussion}

Porphyrins typically adopt planar conformations retaining the aromatic properties of the molecule. However, metal chelation in the center of the ring, between the tetrapyrrole subunits, can result in significant conformational shifts, as observed previously by X-ray

crystallography. ${ }^{59-66}$ In the gas phase, OEP can be ionized using APPI while forming protonated molecules, $[\mathrm{M}+\mathrm{H}]^{+}$, as well as radical ions $[\mathrm{M}]^{+\bullet}$ as in the case of Mn-OEP. When OEP is chelated with a metal, there is a significant mass shift as well as changes in the isotopic ratio of the molecule, generating a unique mass signal that can be detected using FT-ICR MS (see Table 4.1). In combination with TIMS, the structural changes as a function of the chelating agent, such as the increase in mass and volume, can also be studied by measuring their CCS (see Figure 4.1). For example, in comparison of the free OEP and the Mn-, Zn-, and Ni-complexed OEP, an increase in the CCS is observed mainly attributed to the increase in mass and volume. Moreover, in comparison of the $\mathrm{V}=\mathrm{O}$ - and $\mathrm{Ti}=\mathrm{O}$ complexed OEPs, the differences in CCSs are mainly attributed to structural changes because the mass difference is relatively small. 

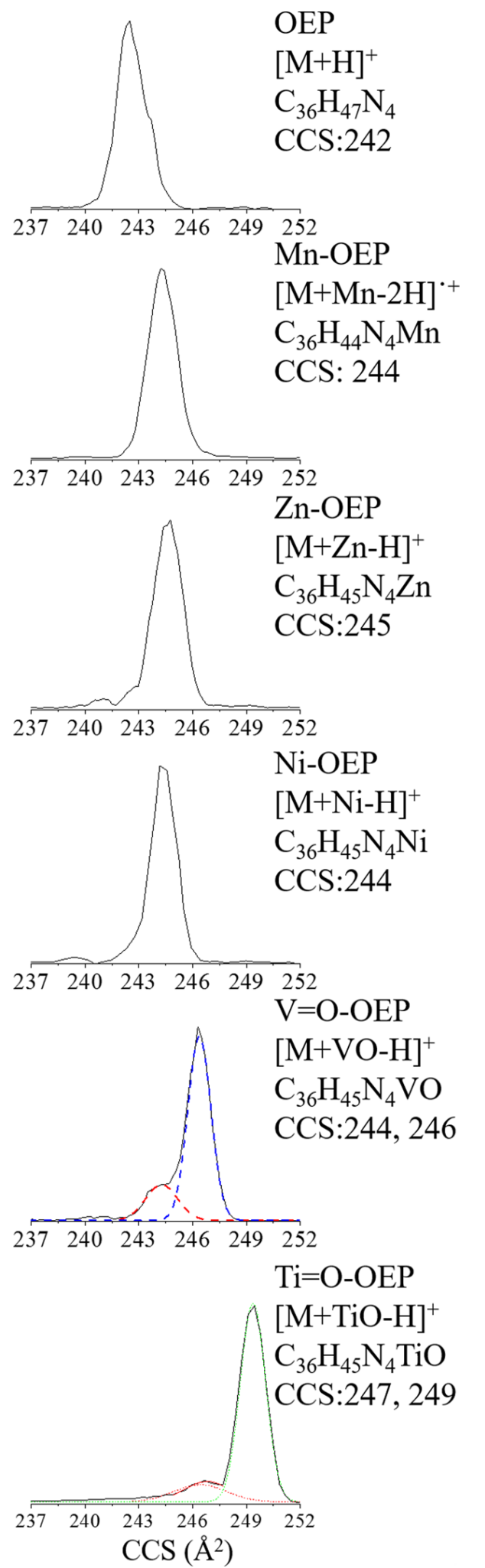

Figure 4.1. Mobility spectra of Me-OEP obtained using APPI-TIMS-FT-ICR MS. Note the single- and dual-band mobility profiles observed for $\mathrm{Mn}, \mathrm{Zn}$, and Ni compared to the vanadyl $(\mathrm{V}=\mathrm{O})$ and titanyl $(\mathrm{Ti}=\mathrm{O})$, respectively. 
An advantage of the TIMS-FT-ICR MS analysis is the possibility to generate chemical formulas using the high mass accuracy of FT-ICR MS and to measure accurate mobility (or CCS) values using TIMS in a single analysis of a complex mixture. Previous results have shown that the combination of FT-ICR MS with TIMS allows for the identification of a greater number of molecular features, with either targeted $^{51}$ or unsupervised $^{53}$ analysis of complex mixtures based on their isomeric content. That is, the high isomeric content of complex mixtures (e.g., crude oils) requires the use of complementary, gas-phase separation tools based on size and mass. The advantage of TIMS-FT-ICR MS analysis for the case of ME-OEP in a complex mixture is shown in Figure 4.2. The primary heteroatom classes of the coal tar mixture $(\mathrm{HC}, \mathrm{N}, \mathrm{O}$, and $\mathrm{S}$ classes) can be distinguished by the space occupied in the two-dimensional (2D) IMS-MS plot by the different classes shown as the dependence of $1 / K_{0}$ (which scales almost linearly with size for a given family) with $m / z$. The position in the 2D IMS-MS plot of each class corresponds to a particular structural characteristic; for example, the nitrogen species occupy primarily a region with higher aliphatic content, double bond equivalent (DBE) of

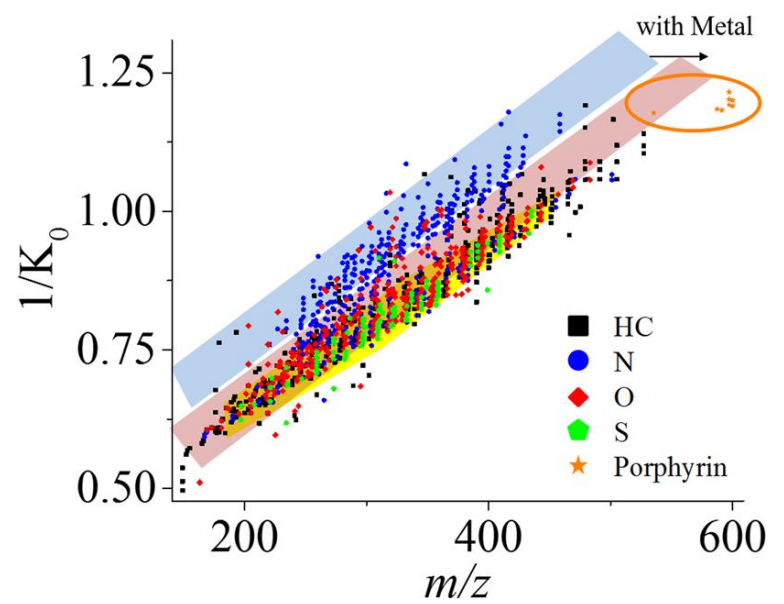

Figure 4.2. Distribution of four commonly encountered molecular classes (HC, N, O, and S) observed in the coal tar APPI-TIMS-FTICR MS analysis and the OEP unique space based on their $m / z$ and inverse mobility $1 / \mathrm{K} 0$. Note the area occupied by OEPs is shifted to a greater $\mathrm{m} / \mathrm{z}$ than other molecules within the aromatic region of the 2D IMS-MS plot. 


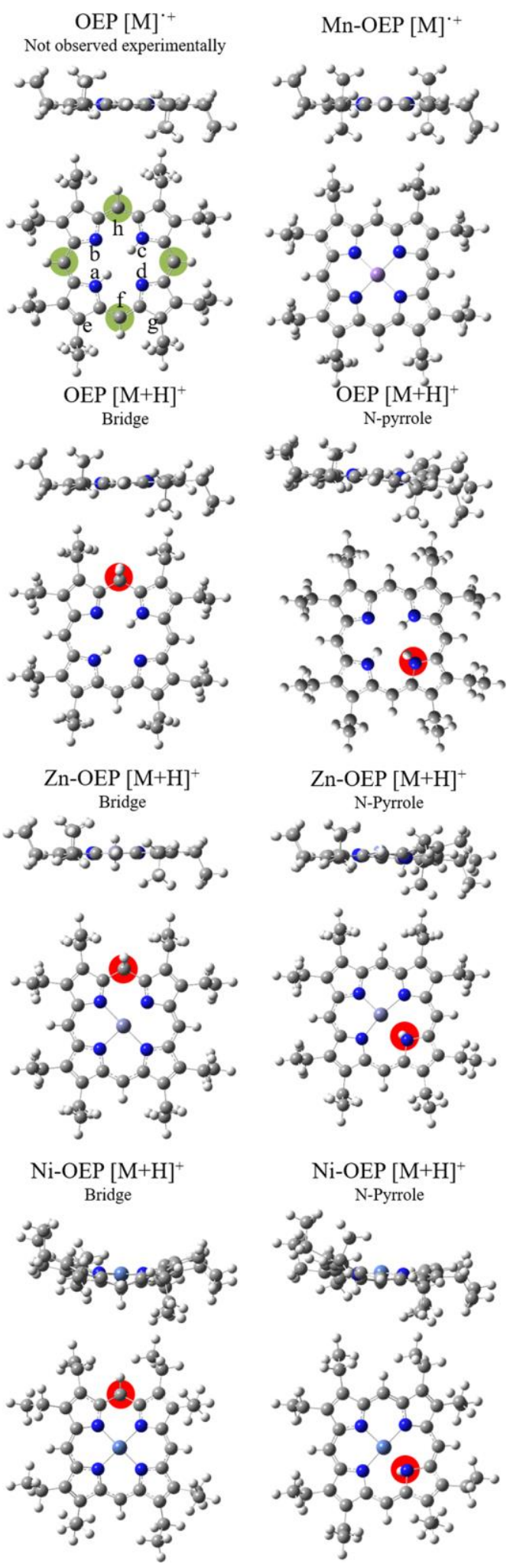

Figure 4.3. Projections of the three-dimensional (3D)-optimized structures for the different OEPs. The radical OEP, not observed experimentally, is labeled with the bridge carbons (green circles) as well as the notation for the distances and angles shown in Table 4.2. Protonation sites are highlighted in red circles. Note that the deviations from planarity depend upon the protonation site. More details can be found in Table 4.2. 
3.5-7.5, while the other three molecular classes, $\mathrm{HC}, \mathrm{O}$, and $\mathrm{S}$, fall within a more aromatic region. While free OEP falls within the trend lines for polyaromatic hydrocarbons, the metal chelator in OEP produces a significant mass shift in comparison to the mobility shift. That is, the metal-complexed OEPs fall out of trend in the 2D IMS-MS plot, which facilitates their identification within the complex mixture of polyaromatic hydrocarbons.

The changes in the Me-OEP structure and size as a function of the metal chelator can be further explored by comparison to candidate structures (Figure 4.3). Of the different structures examined for OEP, only the radical ion form $[\mathrm{M}]^{+}$OEP adopts a true planar conformation in the gas phase; however, the protonated form $[\mathrm{M}+\mathrm{H}]^{+}$was favored in the APPI-TIMS-FT-ICR MS experiments. Two potential protonation sites were considered

Table 4.2. Results from the Theoretical Calculations, Illustrating the Changes in Bond Length between the Nitrogen Atoms in Pyrrole (See Figure 4.3 for Labels), the Bridge Carbons, the Dihedral Angle between Nitrogen, and the Angle between the Bridge ${ }^{\mathrm{a}}$

\begin{tabular}{|c|c|c|c|c|}
\hline & $\overleftrightarrow{a d}(\AA)$ & $\overleftrightarrow{f h}(\AA)$ & $\angle a b c d(\mathrm{deg})$ & $\angle e f g(\mathrm{deg})$ \\
\hline OEP & 4.04 & 6.86 & 0.0 & 176 \\
\hline \multirow[t]{2}{*}{ OEP bridge } & 4.09 & 7.00 & 0.1 & 175 \\
\hline & 4.2 & & & \\
\hline OEP pyrrole & 4.11 & 6.88 & 1.2 & 176 \\
\hline $\mathrm{Mn}-\mathrm{OEP}$ & 3.92 & 6.83 & 0.1 & 178 \\
\hline \multirow[t]{2}{*}{$\mathrm{Zn}-\mathrm{OEP}$ bridge } & 3.83 & 6.93 & 0.7 & $175-9$ \\
\hline & & 6.83 & & \\
\hline \multirow[t]{2}{*}{$\mathrm{Zn}$-OEP pyrrole } & 3.93 & 6.87 & 0.0 & $172-3$ \\
\hline & 4.30 & & & \\
\hline \multirow[t]{2}{*}{$\mathrm{Ni}-\mathrm{OEP}$ bridge } & 3.83 & 6.50 & 0.1 & 143 \\
\hline & & 6.51 & & 163 \\
\hline \multirow[t]{2}{*}{$\mathrm{Ni}-\mathrm{OEP}$ pyrrole } & 3.94 & 6.73 & 0.2 & $163-6$ \\
\hline & 3.86 & & & \\
\hline \multirow[t]{2}{*}{ VO-OEP pyrrole } & 4.01 & 6.87 & 4.5 & $172-6$ \\
\hline & 4.18 & & & \\
\hline \multirow[t]{2}{*}{ VO-OEP bridge } & 4.07 & 6.91 & 0.6 & 163 \\
\hline & & 6.81 & & $173-6$ \\
\hline VO-OEP oxygen & 3.97 & 6.83 & 2.7 & 177 \\
\hline \multirow[t]{2}{*}{ TiO-OEP pyrrole } & 4.06 & 6.89 & 4.5 & 170 \\
\hline & 4.24 & & & 176 \\
\hline \multirow[t]{2}{*}{ TiO-OEP bridge } & 4.12 & 6.83 & 0.5 & 166 \\
\hline & & 6.93 & & $174-6$ \\
\hline TiO-OEP oxygen & 4.01 & 6.85 & 0.0 & 176 \\
\hline
\end{tabular}

${ }^{a}$ Note how each metal and protonation side greatly affects the conformation of the porphyrin. 
for the free OEP: (i) the carbon that bridges the two pyrrole groups and (ii) a free nitrogen on a pyrrole group. Optimization of the gas-phase structures for both compounds show deviations from planarity, which are evaluated on the basis of the symmetry of the distances between the pyrrole subunits, bridge carbons, dihedral angle between the nitrogen atoms, and angle of the bridge carbon (see Table 4.2). For the bridge-bound protonation site, changing the hybridization to $\mathrm{sp}^{3}$ causes the carbon to slightly "pucker", increasing the distance from the opposite carbon from 6.9 to $7.0 \AA$ A . Binding of free nitrogen induces a much greater structural shift because of steric hindrance, which is due to the addition of a proton within the pocket of OEP. This is observed by the change in the dihedral angle between the nitrogen atoms, which are coplanar in the radical OEP $[\mathrm{M}]^{+}$and $0^{\circ}$ and $1^{\circ}$ in the protonated OEP $[\mathrm{M}+\mathrm{H}]^{+}$at the bridge and nitrogen, respectively. The distance between the opposite nitrogen atoms increases from 4.0 to $4.1 \AA$ after protonation. Despite the differences in conformation, both protonated OEP $[\mathrm{M}+\mathrm{H}]^{+}$forms yield a CCS of $248 \AA^{2}$, which is $1-3 \AA^{2}$ greater than the CCS of the radical ion OEP $[\mathrm{M}]^{+}$form. Note that theoretical deviations from the experimental measurement with the IMoS 70\% DHHS model are typically $\sim 2 \%$. While we cannot discriminate the protonation site based on the CCS values, the protonation of nitrogen is energetically more favorable than the bridge in the metal free OEP by $68 \mathrm{~kJ} / \mathrm{mol}$.

Metal binding within the aromatic ring can be challenging to characterize as a result of the insertion of a bulky metal with access to $3 \mathrm{~d}$ orbitals. Chemically, the binding of the metal is accompanied by the loss of the two hydrogens found within the ring and metal complexation within this pocket formed by the tetrapyrrole unit. Of all of the different 
OEPs studied, the manganese OEP was the only porphyrin for which the radical species was observed. Optimized candidate structures show that the Mn-OEP porphyrin has a planar conformation, with symmetric distances between the pyrrole nitrogen atoms $(d=$ $6.8 \AA$ ) and a dihedral angle of $0^{\circ}$. The conformations of $\mathrm{Zn}-\mathrm{OEP}$ and $\mathrm{Ni}-\mathrm{OEP}$ depend primarily upon the metal rather than the protonation site. For example, $\mathrm{Zn}-\mathrm{OEP}$ has a mostly planar conformation independent of whether nitrogen or bridge carbon is protonated. Pyrrole protonation is energetically very similar to protonation of the bridge, with the bridge form being $0.18 \mathrm{~kJ} / \mathrm{mol}$ energetically less favorable than pyrrole. However, protonation of pyrrole induces asymmetry within the molecule, increasing the distances between protonated nitrogen and the opposite nitrogen atom to 4.3 and 3.9 A compared to the bridge protonated structure that has a distance of $4.1 \AA$ between the opposite nitrogen atoms. In comparison to $\mathrm{Mn}-\mathrm{OEP}$ and $\mathrm{Zn}-\mathrm{OEP}$, the nickel metal is complexed much more tightly, as evidenced by the distances between the nitrogens of $3.8 \AA$ with a protonated bridge and $3.9 \AA$ for the protonated nitrogen in Ni-OEP. Because the distances between the opposite nitrogen atoms are smaller in $\mathrm{Ni-OEP,} \mathrm{the} \mathrm{structure} \mathrm{adopts} \mathrm{a} \mathrm{"ruffled"}$ conformation with two bridge carbons pointing upward and two pointing downward. For the bridge protonated structure, the angle between the bridge and pyrroles is reduced to $143^{\circ}$ for the protonated carbon and $163^{\circ}$ for the other bridges. Of the two candidate $\mathrm{Ni}-$ OEP structures, the bridge structure has a lower energy than $\mathrm{N}$ protonated by $28 \mathrm{~kJ} / \mathrm{mol}$.

When free OEP binds to a metal oxide ligand, as in the case of titanyl $(\mathrm{Ti}=\mathrm{O})$ and $\operatorname{vanadyl}(\mathrm{V}=\mathrm{O})$, the high resolving power of TIMS $\left(R_{\mathrm{IMS}} \approx 200\right)$ showed two distinct bands in the mobility spectra. One hypothesis explored was that the two mobility bands could be 
a) Vanadyl-OEP N-pyrrole

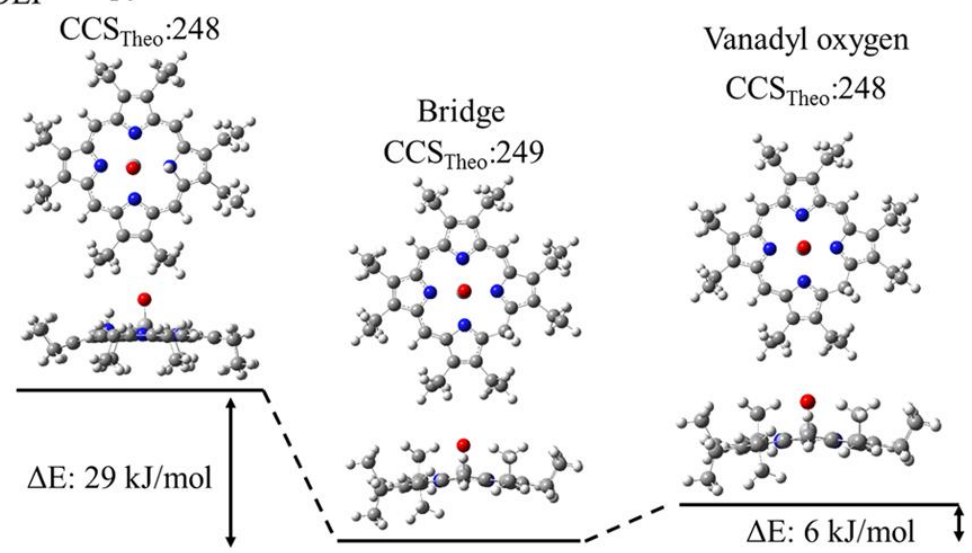

b) Titanyl-OEP N-pyrrole

$$
\mathrm{CCS}_{\text {Theo }}: 250
$$

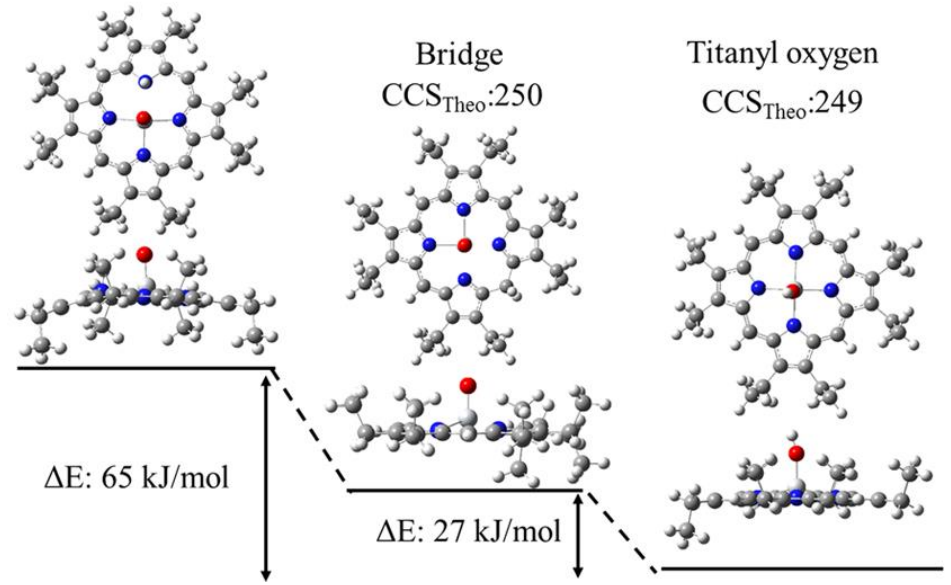

Figure 4.4. Energy landscape diagram showing the change in energy for each protonation site calculated for the vanadyl and titanyl structures. Note that the theoretical CCS are fairly similar for all of the structures, even though each structure is unique and the energies are different.

attributed to two distinct dome conformations, one with the carbonyl pointing out of the dome and a second pointing inward. However, theoretical calculations showed that only the carbonyl pointing out of the dome was energetically favorable and that a local energy minima could not be found when carbonyl was pointing inward, making the theoretical characterization of this state challenging. A second hypothesis explored was that the ionization event simultaneously generates molecular ions with different protonation sites. Three sites were considered, protonating the bridge, nitrogen, and carbonyl (Figure 4.4). Similar to previous results, the protonation of pyrrole nitrogen is the least favorable, while 
the protonation of oxide was significantly more favorable, by approximately $30-65 \mathrm{~kJ} / \mathrm{mol}$. For the vanadyl OEP, the energy differences between the bridge protonation and the oxide are not significantly different and both compounds may be observed experimentally, with a greater relative abundance probably for the lower energy isomer. However, the optimized titanyl OEP structures show a more significant energy barrier between protonated oxide and bridge. Results showed that, although there are significant differences in energy between these structures, the differences in the theoretical CCS do not account for the experimental observations. That is, in both cases, the experimental difference in CCS is between 2 and $3 \AA^{2}$, and the high resolving power of TIMS allows these different states to be resolved. Further improvements on the theoretical CCS calculators are needed when the IMS resolving power exceeds 200 for the analysis of Me-OEP.

These results highlight the potential of fast gas-phase, post-ionization separation and characterization of porphyrins and their different conformations, in either purified or crude samples using TIMS-FT-ICR MS and theoretical calculations. That is, the highresolution ion mobility separation, combined with chemical formula generation based on accurate mass and the theoretical tools, enables the identification and structural characterization of porphyrins as a function of the metal chelate or other modifications to the ring that may cause conformational shifts. Notice that this methodology can be further adapted for the quantification of Me-OEP within complex mixtures in a single analysis without the need for pre-fractionation. 


\subsection{Conclusions}

This work illustrates the use of TIMS-FT-ICR MS for the gas-phase separation and characterization of free OEP and OEP complexed with manganese, nickel, zinc, vanadyl, and titanyl. Upon metal binding, changes in CCS are observed as a function of the metal complexed within the pocket of the OEP ring, with single, high resolving power $\left(R_{\mathrm{IMS}} \sim\right.$ 150-250) bands observed for $\mathrm{OEP}\left(\mathrm{H}_{2}, \mathrm{Mn}, \mathrm{Ni}\right.$, and $\left.\mathrm{Zn}\right)$, while two IMS bands are observed for the metal oxides vanadyl and titanyl OEP. Experimental results from APPI-TIMS-FTICR MS show that the metal-complexed OEPs have unique shifts in $\mathrm{m} / \mathrm{z}$ and CCS, which allows for their identification within a complex mixture of aromatic molecules from coal tar. The shifts in collisional cross section for Me-OEP as a function of Me suggest that there are significant conformational changes upon metal binding. Candidate structures were proposed for the different Me-OEP as a function of the Me and protonation site. A good agreement is observed between the most energetically favorable candidate structures

and the measured CCS. Results also show the need for the development of new and better CCS calculators ( $<2 \%$ error) when using high-resolution IMS instrumentation, such as TIMS. This work provides the proof of principle for fast identification and characterization of the conformational space of Me-OEP within complex mixtures.

\subsection{References}

1.Treibs, A. Liebigs Ann. Chem. 1935, 520 (1), 144-150.

2.Treibs, A. Liebigs Ann. Chem. 1934, 509 (1), 103-114.

3.Treibs, A. Justus Liebigs Ann. Chem. 1934, 510 (1), 42-62.

4.Treibs, A.; Dinelli, D. Liebigs Ann. Chem. 1935, 517 (1), 170-172. 
5.Treibs, A. Angew. Chem. 1936, 49 (38), 682-686.

6.Eglinton, G.; Maxwell, J. R.; Evershed, R. P.; Barwise, A. J. G. Interdiscip. Sci. Rev. 1985, 10 (3), 222-236.

7.Ocampo, R.; Riva, A.; Trendel, J. M.; Riolo, J.; Callot, H. J.; Albrecht, P. Energy Fuels 1993, 7 (2), 191-193.

8.Philp, R. P. Oil-Oil and Oil- Source Rock Correlations: Techniques. In Organic Geochemistry; Engel, M. H., Macko, S. A., Eds.; Springer: Boston, MA, 1993; Vol. 11, Chapter 19, pp 445-460.

9.Quirke, J. M. E.; Dale, T.; Britton, E. D.; Yost, R. A.; Trichet, J.; Belayouni, H. Org. Geochem. 1990, 15 (2), 169-177.

10. Bonnett, R.; Burke, P. J.; Czechowski, F. Metalloporphyrins in Lignite, Coal, and Calcite. In Metal Complexes in Fossil Fuels ; Filby, R. H., Branthaver, J. F., Eds.; American Chemical Society (ACS): Washington, D.C., 1987; ACS Symposium Series, Vol. 344, Chapter 11, pp 173-185.

11. Kashiyama, Y.; Ogawa, N. O.; Nomoto, S.; Kitazato, H.; Ohkouchi, N.Earth, Life, Isot. $2010,313-335$.

12. Quirke, J. M. E. Rationalization for the Predominance of Nickel and Vanadium Porphyrins in the Geosphere. In Metal Complexes in Fossil Fuels; Filby, R. H., Branthaver, J. F., Eds.; American Chemical Society (ACS): Washington, D.C., 1987; ACS Symposium Series, Vol.344, Chapter 4, pp 74-83.

13. Sugihara, J. M.; Bean, R. M. J. Chem. Eng. Data 1962, 7 (2), 269-271.

14. Buenrostro-Gonzalez, E.; Andersen, S. I.; Garcia-Martinez, J. A.; Lira-Galeana, C. Energy Fuels 2002, 16 (3), 732-741.

15. Biggs, W. R.; Fetzer, J. C.; Brown, R. J.; Reynolds, J. G. Liq. Fuels Technol. 1985, 3 (4), 397-421.

16. Zhao, X.; Xu, C.; Shi, Q. Porphyrins in Heavy Petroleums: A Review. In Structure and Modeling of Complex Petroleum Mixtures; Xu, C., Shi, Q., Eds.; Springer: Berlin, Germany, 2015; Vol. 168 ,pp39-70.

17. Zhao, X.; Liu, Y.; Xu, C. M.; Yan, Y. Y.; Zhang, Y. H.; Zhang, Q. Y.; Zhao, S. Q.; Chung, K.; Gray, M. R.; Shi, Q. Energy Fuels 2013, 27 (6), 2874-2882.

18. Kekalainen, T.; Pakarinen, J. M. H.; Wickstrom, K.; Lobodin, V. V.; McKenna, A. M.; Janis, J. Energy Fuels 2013, 27 (4), 2002-2009. 
19. McKenna, A. M.; Purcell, J. M.; Rodgers, R. P.; Marshall, A. G. Energy Fuels 2009, 23 (4), 2122-2128.

20. Qian, K.; Edwards, K. E.; Mennito, A. S.; Walters, C. C.; Kushnerick, J. D. Anal. Chem. 2010, 82 (1), 413-9.

21. Swider, P.; Lewtak, J. P.; Gryko, D. T.; Danikiewicz, W. J. Mass Spectrom. 2013, 48 (10), 1116-24.

22. McKenna, A. M.; Williams, J. T.; Putman, J. C.; Aeppli, C.; Reddy, C. M.; Valentine, D. L.; Lemkau, K. L.; Kellermann, M. Y.; Savory, J. J.; Kaiser, N. K.; Marshall, A. G.; Rodgers, R. P. Energy Fuels 2014, 28 (4), 2454-2464.

23. Romao, W.; Tose, L. V.; Vaz, B. G.; Sama, S. G.; Lobinski, R.; Giusti, P.; Carrier, H.; Bouyssiere, B. J. Am. Soc. Mass Spectrom. 2016, 27 (1), 182-5.

24. Ren, L. M.; Han, Y. H.; Zhang, Y. H.; Zhang, Y. F.; Meng, X. H.; Shi, Q. Energy Fuels 2016, 30 (6), 4486-4493.

25. Cho, Y.; Witt, M.; Jin, J. M.; Kim, Y. H.; Nho, N. S.; Kim, S. Energy Fuels 2014, 28 (11), 6699-6706.

26. Kim, E.; Cho, E.; Moon, S.; Park, J. I.; Kim, S. Energy Fuels 2016, 30 (4), 2932-2940.

27. Kaczorowska, M. A.; Cooper, H. J. Chem. Commun. 2011, 47 (1), 418-20.

28. Charkin, O. P.; Klimenko, N. M.; Nguyen, P. T.; Charkin, D. O.; Mebel, A. M.; Lin, S. H.; Wang, Y. S.; Wei, S. C.; Chang, H. C. Chem. Phys. Lett. 2005, 415 (4-6), 362-369.

29. Fernandez-Lima, F. A.; Becker, C.; McKenna, A. M.; Rodgers, R.P.; Marshall, A. G.; Russell, D. H. Anal. Chem. 2009, 81 (24), 9941-7.

30. Fasciotti, M.; Lalli, P. M.; Klitzke, C. F.; Corilo, Y. E.; Pudenzi, M. A.; Pereira, R. C. L.; Bastos, W.; Daroda, R. J.; Eberlin, M. N. Energy Fuels 2013, 27 (12), 7277-7286.

31. Lalli, P. M.; Corilo, Y. E.; Rowland, S. M.; Marshall, A. G.; Rodgers, R. P. Energy Fuels 2015, 29 (6), 3626-3633.

32. Ahmed, A.; Cho, Y. J.; No, M. H.; Koh, J.; Tomczyk, N.; Giles, K.; Yoo, J. S.; Kim, S. Anal. Chem. 2011, 83 (1), 77-83.

33. Ahmed, A.; Cho, Y.; Giles, K.; Riches, E.; Lee, J. W.; Kim, H. I.; Choi, C. H.; Kim, S.Anal. Chem. 2014, 86 (7), 3300-3307.

34. Ponthus, J.; Riches, E. Int. J. Ion Mobility Spectrom. 2013, 16 (2), 95-103. 
35. Santos, J. M.; Galaverna, R. D.; Pudenzi, M. A.; Schmidt, E. M.; Sanders, N. L.; Kurulugama, R. T.; Mordehai, A.; Stafford, G. C.;Wisniewski, A.; Eberlin, M. N. Anal. Methods 2015, 7 (11), 4450-4463.

36. Fernandez-Lima, F. A.; Blase, R. C.; Russell, D. H. Int. J. Mass Spectrom. 2010, 298 (1-3), 111-118.

37. Fernandez-Lima, F. A.; Wei, H.; Gao, Y. Q.; Russell, D. H. J.Phys. Chem. A 2009, 113 (29), 8221-8234.

38. Valentine, S. J.; Counterman, A. E.; Clemmer, D. E. J. Am. Soc. Mass Spectrom.1999, 10 (11), 1188-1211.

39. Tao, L.; McLean, J. R.; McLean, J. A.; Russell, D. H. J. Am. Soc. Mass Spectrom. 2007, 18 (7), 1232-8.

40. Bush, M. F.; Hall, Z.; Giles, K.; Hoyes, J.; Robinson, C. V.; Ruotolo, B. T. Anal. Chem. 2010, 82 (22), 9557-65.

41. Jarrold, M. F. Annu. Rev. Phys. Chem. 2000, 51 (1), 179-207.

42. Murray, M. M.; Krone, M. G.; Bernstein, S. L.; Baumketner, A.; Condron, M. M.; Lazo, N. D.; Teplow, D. B.; Wyttenbach, T.; Shea, J. E.; Bowers, M. T. J. Phys. Chem. B 2009, 113 (17), 6041-6.

43. Baumketner, A.; Bernstein, S. L.; Wyttenbach, T.; Bitan, G.; Teplow, D. B.; Bowers, M. T.; Shea, J. E. Protein Sci. 2006, 15 (3), 420-428.

44. Castellanos, A.; Benigni, P.; Hernandez, D. R.; DeBord, J. D.; Ridgeway, M. E.; Park, M. A.; Fernandez-Lima, F. Anal. Methods 2014, 6 (23), 9328-9332.

45. McKenzie-Coe, A.; DeBord, J. D.; Ridgeway, M.; Park M.; Eiceman, G.; Fernandez-Lima, F. Analyst 2015, 140 (16), 5692-9.

46. Adams, K. J.; Montero, D.; Aga, D.; Fernandez-Lima, F. Int. J. Ion Mobility Spectrom. 2016, 19 (2-3), 69-76.

47. Schenk, E. R.; Almeida, R.; Miksovska, J.; Ridgeway, M. E.; Park, M. A.; Fernandez-Lima, F.J. Am. Soc. Mass Spectrom. 2015, 26 (4), 555-63.

48. Schenk, E. R.; Mendez, V.; Landrum, J. T.; Ridgeway, M. E.; Park, M. A.; Fernandez-Lima, F. Anal. Chem. 2014, 86 (4), 2019-24.

49. Schenk, E. R.; Ridgeway, M. E.; Park, M. A.; Leng, F.; Fernandez-Lima, F. Anal. Chem. 2014, 86 (2), 1210-4. 
50. Benigni, P.; Marin, R.; Molano-Arevalo, J. C.; Garabedian, A.; Wolff, J. J.; Ridgeway, M. E.; Park, M. A.; Fernandez-Lima, F. Int. J. Ion Mobility Spectrom. 2016, 19 (2-3), 95-104.

51. Benigni, P.; Thompson, C. J.; Ridgeway, M. E.; Park, M. A.; Fernandez-Lima, F. Anal. Chem. 2015, 87(8), 4321-5.

52. Pu, Y.; Ridgeway, M. E.; Glaskin, R. S.; Park, M. A.; Costello, C. E.; Lin, C. Anal. Chem. 2016, 88 (7), 3440-3.

53. Benigni, P.; Fernandez-Lima, F. Anal. Chem. 2016, 88 (14), 7404-12.

54. Buchler, J. W.; Eikelmann, G.; Puppe, L.; Rohbock, K.; Schneehage, H. H.; Weck, D. Liebigs Ann. Chem. 1971, 745 (1), 135-151.

55. Flanagan, L.A. Mass Spectrometry Calibration Using Homogeneously Substituted Fluorinated Triazatriphosphorines. U.S. Patent 5,872,357 A, Feb 16, 1999; pp 19.

56. Fernandez-Lima, F. A.; Kaplan, D. A.; Park, M. A. Rev. Sci. Instrum.2011, 82 (12), 126106.

57. Fernandez-Lima, F.; Kaplan, D. A.; Suetering, J.; Park, M. A. Int. J. Ion Mobility Spectrom. 2011, 14 (2-3), 93-98.

58. McDaniel, E. W.; Mason, E. A. Mobility and Diffusion of Ions in Gases; John Wiley and Sons, Inc.: New York, 1973; pp 381.

59. Cheng, B.; Scheidt, W. R. Acta Crystallogr., Sect. C: Cryst. Struct. Commun. 1995, $51(5), 825-828$.

60. Molinaro, F. S.; Ibers, J. A. Inorg. Chem. 1976, 15 (9), 2278-2283.

61. Meyer, E. F., Jr. Acta Crystallogr., Sect. B: Struct. Crystallogr. Cryst. Chem. 1972, 28 (7), 2162-2167.

62. Cullen, D. L.; Meyer, E. F. J. Am. Chem. Soc. 1974, 96 (7), 2095-2102.

63. Brennan, T. D.; Scheidt, W. R.; Shelnutt, J. A. J. Am. Chem. Soc. 1988, 110 (12), 3919-3924.

64. Guilard, R.; Latour, J. M.; Lecomte, C.; Marchon, J. C.; Protas, J.; Ripoll, D. Inorg. Chem.1978, 17 (5), 1228-1237.

65. Lauher, J. W.; Ibers, J. A. J. Am. Chem. Soc. 1973, 95 (16), 5148-52. 
66. Cullen, D. L.; Meyer, E. F., Jr. Acta Crystallogr., Sect. B: Struct. Crystallogr. Cryst. Chem. 1976, 32 (8), 2259-2269.

67. Montgomery, J. A., Jr; Frisch, M. J.; Ochterski, J. W.; Petersson, G. A. J. Chem. Phys. 1999, 110 (6), 2822-2827.

68. Singh, U. C.; Kollman, P. A. J. Comput. Chem. 1984, 5 (2), 129-145.

69. Besler, B. H.; Merz, K. M.; Kollman, P. A. J. Comput. Chem. 1990, 11 (4), 431-439.

70. Larriba, C.; Hogan, C. J. J. Comput. Phys. 2013, 251, 344-363.

71. Larriba, C.; Hogan, C. J., Jr. J. Phys. Chem. A 2013, 117 (19), 3887-901.

72. Larriba-Andaluz, C.; Fernandez-Garcia, J.; Ewing, M. A.; Hogan, C. J., Jr.; Clemmer, D. E. Phys. Chem. Chem. Phys. 2015,17 (22), 15019-29 


\section{CHAPTER V}

TOWARDS UNSUPERVISED POLYAROMATIC HYDROCARBONS STRUCTURAL ASSIGNMENT FROM SA-TIMS -FTMS DATA

(Adapted from Benigni et al., 2015, Int J Ion Mobil Spectrom, with permission of Springer) 


\subsection{Abstract}

With the advent of high resolution ion mobility analyzers and their coupling to ultrahigh resolution mass spectrometers, there is a need to further develop a theoretical workflow capable of correlating experimental accurate mass and mobility measurements with tridimensional candidate structures. In the present work, a general workflow is described for unsupervised tridimensional structural assignment based on accurate mass measurements, mobility measurements, in silico 2D-3D structure generation, and theoretical mobility calculations. In particular, the potential of this workflow will be shown for the analysis of polyaromatic hydrocarbons from Coal Tar SRM 1597a using selected accumulation - trapped ion mobility spectrometry (SA-TIMS) coupled to Fourier transform- ion cyclotron resonance mass spectrometry (FT-ICR MS). The proposed workflow, which can be adapted to different IMS scenarios, can utilize different collisional cross-section calculators and has the potential to include $\mathrm{MS}^{\mathrm{n}}$ and $\mathrm{IMS}^{\mathrm{n}}$ measurements for faster and more accurate tridimensional structural assignment.

\subsection{Introduction}

Over the last 20 years, there has been an increase in the use of ion mobility spectrometry (IMS) for rapid analysis and separation of isomers, ${ }^{1-4}$ biological conformers, ${ }^{5-}$ ${ }^{7}$ and species of differing chemical classes based on differences in functional groups, polarities, and atomic composition. ${ }^{8,9}$ Most of these efforts have been driven by the development of higher resolution and more sensitive variants of IMS (e.g., periodic focusing DC ion guide, ${ }^{10-12}$ segmented quadrupole drift cell,,${ }^{13}$ multistage IMS, ${ }^{14-16}$ field asymmetric IMS, ${ }^{17}$ traveling wave ion guide, ${ }^{18-19}$ and trapped ion mobility spectrometers, $(\text { TIMS })^{20-22}$ ). One advantage of TIMS is the ability to trap a mobility range of interest, 
explore molecular dynamics, ${ }^{23}$ kinetic intermediates, ${ }^{6,24}$ and to follow structural changes during the IMS time scale under controlled experimental condition (e.g., reactive/inert, polar/nonpolar bath gas at different temperatures). ${ }^{25}$ In addition, TIMS can be run in selective accumulation mode, which facilitates its coupling to ultrahigh resolution mass analyzers (SA-TIMS-FTMS). ${ }^{26}$ That is, SA-TIMS-FTMS can provide accurate mobility, exact mass measurements, and when complemented with reference standard measurements and/or theoretical calculations it permits direct structural assignment.

In the present paper, a general workflow is describe for unsupervised tridimensional structural assignment using accurate mass measurements, mobility measurements, in silico 2D-3D structural generation, and theoretical mobility calculations. In particular, the potential of this workflow will be shown for the analysis of polyaromatic hydrocarbons (PAH) from a Coal Tar (SRM 1597a) analysis using SA-TIMS-FTMS. The proposed workflow can be adapted to different IMS scenarios, different ion-neutral collisional crosssection (CCS) calculators and has the potential to include $\mathrm{MS}^{\mathrm{n}}$ and IMS ${ }^{\mathrm{n}}$ measurements for faster and more accurate structural assignment.

\subsection{Experimental Section}

\subsubsection{Sample Preparation}

A "Complex mixture of Polyaromatic hydrocarbons Coal Tar in oil" SRM1597a was obtained from the National Institute of Standards and Technology (Baltimore, MA) and used as received. SRM1597a was dissolved in 1:1 v:v optima grade methanol:toluene (Fisher Scientific, Waltham, MA) and diluted 1:100 prior to analysis. 


\subsubsection{SA-TIMS-FTMS analysis}

Details regarding TIMS operation and specifics compared to traditional IMS can be found elsewhere. ${ }^{2,6,20,21,22}$ Briefly, TIMS mobility separation is based on holding the ions stationary using an electric field (E) against a moving gas. In traditional TIMS operation, multiple geometric isomers/conformers are trapped simultaneously at different E values resulting from a voltage gradient applied across the TIMS tunnel (more details in 21, 23). The E gradient defines the IMS range that is trapped and analyzed, thus allowing low resolution (large E gradient) and high resolution (small E gradient) IMS separations. The possibility to separate and accumulate single isomers/conformers over time in a TIMS device relies on selecting the E gradient, and by performing stepwise elutions into the mass analyzers by reducing the voltage range within a single trapping step. Multi-step elutions are typically used when TIMS is coupled to fast acquisition rate MS analyzers (e.g., TOFMS). However, when TIMS is coupled to slower MS analyzers (e.g., FT-ICR MS), TIMS's operation is changed to single step elutions (from a small E gradient that defines the IMS resolution) and sequential scanning of the E gradient range. That is, each isomer/conformer eluting from the IMS cell can be described by a $\mathrm{E} \pm \Delta \mathrm{E}$ value; the smaller the $\Delta \mathrm{E}$ value the higher the IMS resolution and accuracy to determine the reduced mobility $K_{0} \pm \Delta K_{0}$ value. This mode of operation is called selected accumulation trapped ion mobility spectrometry (SA-TIMS). SA-TIMS operation was controlled using in-house software, written in National Instruments Lab VIEW (2012, v. 12.0f3), and synchronized with the FT-ICR MS acquisition program. IMS separation was performed using nitrogen as a bath gas at ca. $300 \mathrm{~K}$, and the gas flow velocity was controlled by the pressure difference between entrance funnel $\mathrm{P}_{1}=2.6 \mathrm{mbar}$, and the exit funnel $\mathrm{P}_{2}=1.3$ mbar. $\mathrm{P}_{1}$ and $\mathrm{P}_{2}$ values were held 
constant for all experiments. The same RF (2080 kHz and 240Vpp) was applied to all electrodes including the entrance funnel, the mobility separating section, and the exit funnel. The SA-TIMS cell was operated using a fill/trap/elute/quench sequence of 250 600/90/25/10 ms, using an average of 30 IMS scans per FTMS spectrum and a voltage difference across the $\Delta \mathrm{E}$ gradient of $0.5-1.0 \mathrm{~V}$. Under these conditions, the average IMS resolution was $70-120$.

An atmospheric pressure laser ionization source (APLI, Bruker Daltonics, Inc., MA) was used for all the analyses. Briefly, the APLI source utilizes a $266 \mathrm{~nm}$ laser (CryLas GmbH, Berlin, Germany; Type:1HP266-50) and allows for sample introduction via an atmospheric pressure heated vaporizer. It should be noted that this source targets polyaromatic hydrocarbon compounds. ${ }^{27-29}$

MS acquisition was optimized for highest transmission in the $m / z 100-600$ in the 7T Solarix FT-ICR MS spectrometer (Bruker Daltonics Inc., MA). SA-TIMS-FTMS spectra were acquired at 2 Mword using half-sine apodization followed by fast-Fourier transform with magnitude mode resolutions of $\mathrm{R} \sim 150 \mathrm{~K}$ at $\mathrm{m} / \mathrm{z} 400$. MS spectra were acquired with 100 scans at 8 Mword with experimental resolutions of $\mathrm{R} \sim 600 \mathrm{k}$ at $m / z 400$. External IMS and MS calibrations were performed utilizing Agilent ESI-ToF tuning mix (Tunemix, G2421A, Agilent Technologies, Santa Clara, CA). The chemical formula calculations from the exact mass domain were performed using Data Analysis (Version 4.2, Bruker Daltonik) with a maximum chemical formula of $\mathrm{C}_{1-100} \mathrm{H}_{1-100} \mathrm{~N}_{0-2} \mathrm{O}_{0-4} \mathrm{~S}_{0-2}$, odd and even electron configurations allowed, and a mass tolerance of $0.5 \mathrm{ppm}$. The number of double bond equivalents (DBE) was calculated using the equation: 


$$
D B E=C-\frac{H}{2}+\frac{N}{2}+1
$$

where $\mathrm{C}, \mathrm{H}$, and $\mathrm{N}$ are the number of the respective elements. Processing of IMS-MS data was performed using in-house scripts written in MATLAB (R2014b, MathWorks Inc., Natick, MA).

\subsubsection{Theoretical methods}

Candidate structures were proposed using the workflow described in the Scheme 5.1. Briefly, chemical formulas are generated from the ultrahigh resolution MS data. For each chemical formula identified in the FTMS spectra, candidate structures are generated from online databases (e.g., ChemSpider and PubChem) and/or from in silico atomistic structure generators (MOLGEN ${ }^{30}$ and STRGEN 231,32). The candidate structures undergo geometry optimization utilizing ab initio density functional theory (DFT) methods, typically using 6-31G basis set for fast geometry optimization. Theoretical ion-neutral collision cross sections are then calculated using MOBCAL for nitrogen ${ }^{33,34}$ and IMoS (v $1.06 \mathrm{dw})^{35-37}$ softwares with a bath gas at ca. $300 \mathrm{~K}$. IMoS calculations were performed

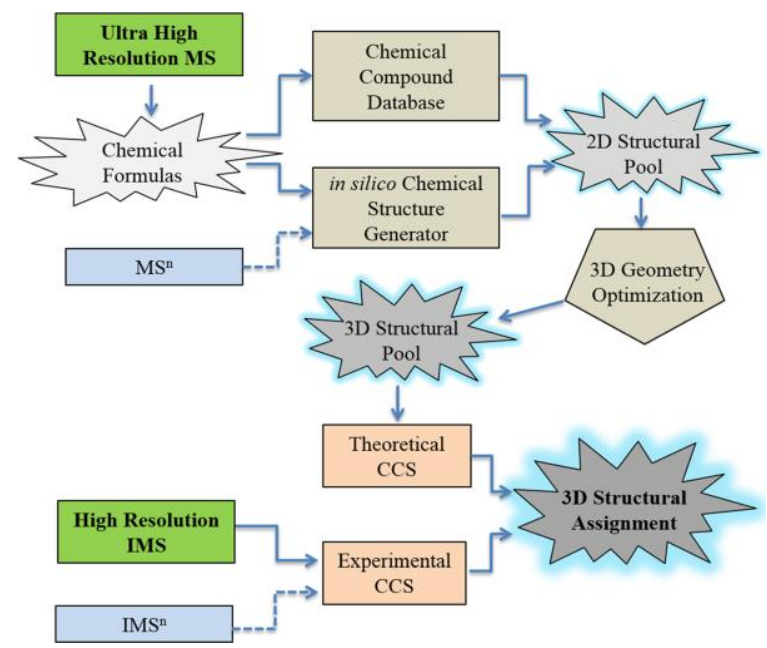

Scheme 5.1. Outline for the theoretical workflow proposed for unsupervised structural assignment from IMS and MS data 
using the trajectory method with 100,000 gas molecules, fully diffuse collisions and a $92 \%$ Maxwell distribution remission velocity. After theoretical CCS are calculated, the results are compared with the experimental values and structural assignment is performed. IMS ${ }^{\mathrm{n}}$ and $\mathrm{MS}^{\mathrm{n}}$ data can be also incorporated (following the same workflow) for higher confidence in the structural assignment.

\subsection{Results and Discussion}

The separation of PAH from a coal tar sample by high resolution ion mobility and ultrahigh resolution mass spectrometry permits the observation of unique molecular signatures based on the chemical formula and the tridimensional structural arrangement (see Fig. 5.1a). Inspection of the IMS-MS contour plot shows two distinct trend lines,
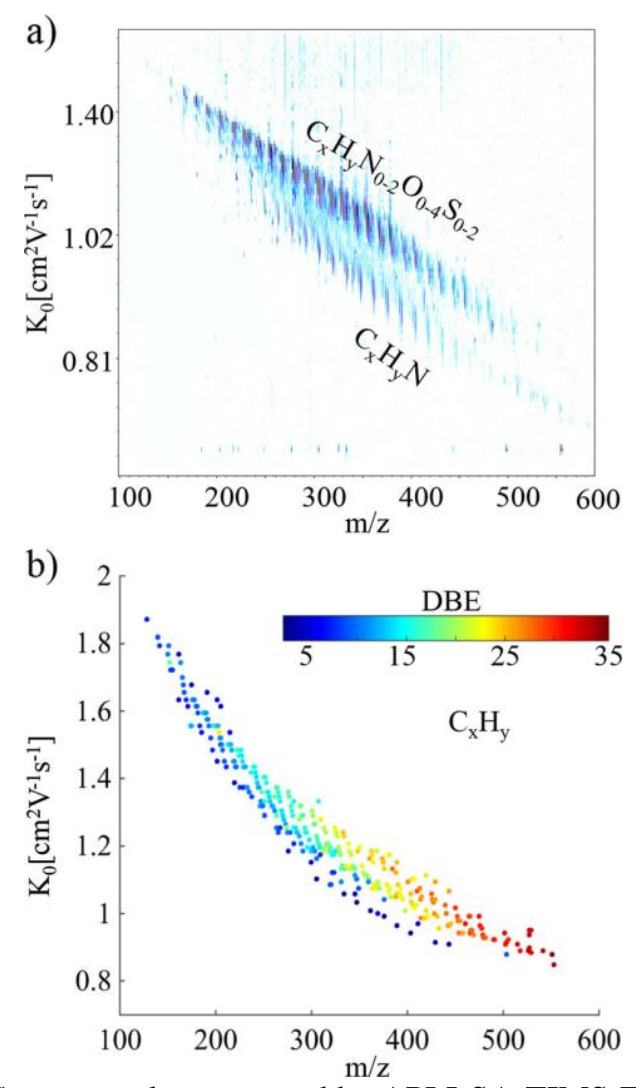

Figure 5.1. a) Typical IMS-MS contour plot generated by APLI-SA-TIMS-FTMS for the Coal Tar SRM and b) Mobility as a function of the $m / z$ (DBE number in colorscale) for the CxHy class. 
corresponding to the separation of the $\mathrm{C}_{\mathrm{x}} \mathrm{H}_{\mathrm{y}} \mathrm{N}_{1}$ class from the $\mathrm{C}_{\mathrm{x}} \mathrm{H}_{\mathrm{y}} \mathrm{N}_{0-2} \mathrm{O}_{0-4} \mathrm{~S}_{0-2}$ classes. Taking into consideration that the APLI source preferentially ionizes aromatic rings, we can further identify the base structure for the $\mathrm{C}_{\mathrm{x}} \mathrm{H}_{\mathrm{y}} \mathrm{N}_{1}$ class according to the DBE, which corresponds to a series of alkylated pyridines and pyrroles.

A unique feature of the SA-TIMS-FTMS analysis is the potential to combine the accurate $m / z, \mathrm{DBE}$ and $\mathrm{K}_{0}$ information in order to make a structural assignment. For example, isolation of peaks corresponding to the $\mathrm{C}_{\mathrm{x}} \mathrm{H}_{\mathrm{y}}$ chemical class (see Fig. 5.1b) permits the inspection of their structural diversity and complexity. That is, closer inspection of Fig. 5.1b shows that as the mass range increases, higher DBE are observed, which translates in highly condensed, aromatic molecules. This observation is in agreement with the 'planar limit' in fossil fuels relating the highest condensed state for a given carbon number. ${ }^{38,39}$
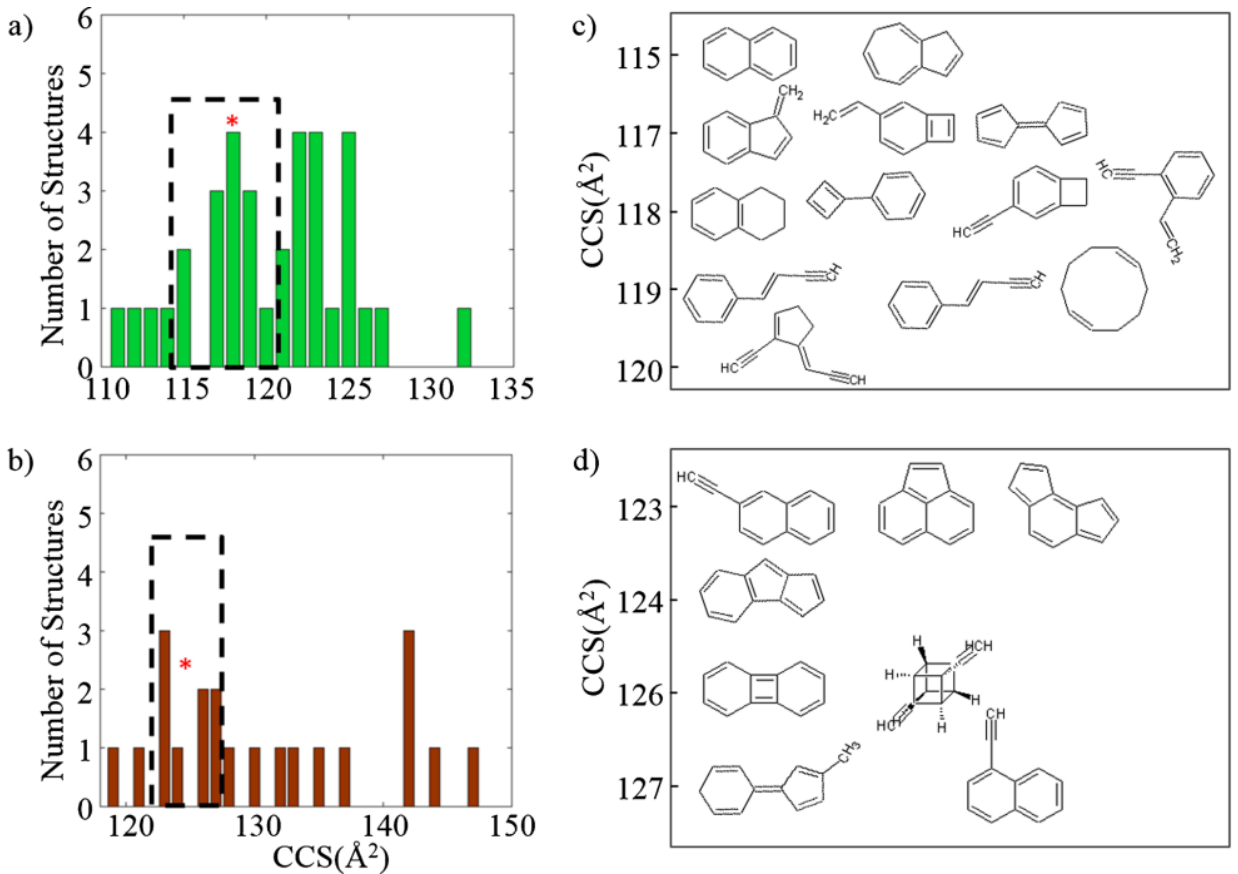

Figure 5.2. Number of structures at nominal CCS for a) $\mathrm{C}_{10} \mathrm{H}_{8}$ and b) $\mathrm{C}_{12} \mathrm{H}_{8}$. Structures within $2 \%$ of the experimental value (*) are shown for $\mathrm{C}_{10} \mathrm{H}_{8}$ and $\mathrm{C}_{12} \mathrm{H}_{8}$ in c) and $\mathrm{d}$ ), respectively. 
A common workflow during IMS-MS analysis is the combination of experimental and theoretical tools for structural assignment. Most of previous studies have focused on the identification of a limited number of mobility peaks. However, in the case of complex samples, the large number of chemical formulas and the structural diversity makes the structural assignment based on accurate mobility, chemical formula and DBE a challenging computational problem. To better illustrate the complexity, the structural diversity of PAH was studied for the case of $\mathrm{C}_{10} \mathrm{H}_{8}$ and $\mathrm{C}_{12} \mathrm{H}_{8}$ using the workflow described in the Scheme 5.1. Considering all the structures included in ChemSpider, the CCS calculations showed that there are multiple candidate structures at nominal CCS (see Fig. 5.2). Moreover, further analysis of the relative stability of the proposed candidate structures shows that if only the lowest energy structures are considered, structural assignment may be feasible (see Appendix 5.1 and 5.2). For example, the lowest energy structures for $\mathrm{C}_{10} \mathrm{H}_{8}$ are naphthalene, 1-methyenelindene, and azulene. In this case 1-methyenelindene can be resolved experimentally from the other two which have the same nominal CCS. A similar trend is observed with acenapthylene, 2-ethynyl-naphthalene, and 1-ethynyl-naphthalene for the formula $\mathrm{C}_{12} \mathrm{H}_{8}$. If compare with the SRM certificate information, naphthalene and acenapthylene assignments are confirmed. ${ }^{40}$ However, structural verification can also be made from the experimental CCS observed. That is, IMS analysis only provided one IMS band for $\mathrm{C}_{10} \mathrm{H}_{8}$ and $\mathrm{C}_{12} \mathrm{H}_{8}$ and the experimental and theoretical CCS agree within $2 \%$ difference (i.e., naphthalene $\mathrm{CCS}_{\exp }=118$ and $\mathrm{CCS}_{\text {calc }}=115$ and acenapthylene $\mathrm{CCS}_{\exp }=125$ and $\left.\mathrm{CCS}_{\text {calc }}=125\right)$. Previous studies correlating theoretical calculations to experimental values have shown that a $3-5 \%$ variation between the experimental and theoretical CCS from known structures can be expected. ${ }^{22,}{ }^{26}$ It should be noted that in 
general, this can be further complemented with $\mathrm{IMS}^{\mathrm{n}}$ and $\mathrm{MS}^{\mathrm{n}}$ data for a more accurate structural assignment.

An important part of the workflow previously described is the search for candidate structures. Although the initial pool of structures can be compiled using existing databases, this approach is limited by the size and diversity of the database. On the other hand, one can better characterize the structural space using an in silico molecular structure generator based solely on the chemical formula. Although this approach looks straightforward, its implementation can be challenging because of the exponential increase in structural diversity with the carbon number (see example for the case of a hydrocarbon series in Fig. 5.3a). The structural complexity with the carbon number can be illustrated by the inspection of the number of structures as a function of DBE for the PAH class (see Fig. 5.3b). An interesting observation is that as the carbon number increases, there is a maxima in the number of structures that can be produced at each DBE. That is, for larger carbon number the DBE imposes constraints in the number of possible structures.

In the workflow proposed, the conversion from 2 to 3 dimensions includes geometry optimization prior to CCS calculations. To shorten the computation time, a small basis set (e.g., DFT/6-31G) is recommended as long as it accurately reproduces the PAH geometry. ${ }^{41,42}$ Although a single geometry optimization can be performed in short timescales using current computing resources (see Fig. 5.3c), the total time scales with the number of atoms (due to longer optimization times). For example, for the chemical formula $\mathrm{C}_{12} \mathrm{H}_{20}$, it would take 31 computational years to calculate all of the potential structures if 
performed in serial mode. Moreover, these calculations can be made over a more realistic time if parallel computing and multimode computer resources are utilized.
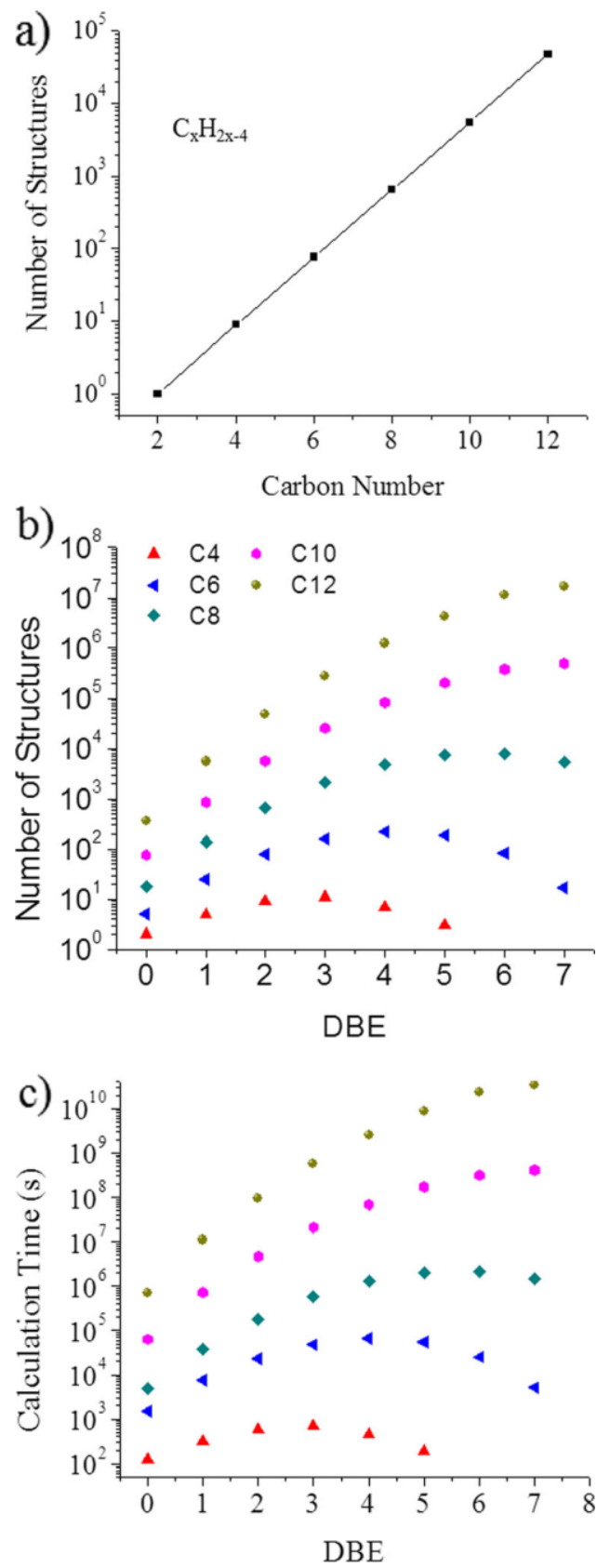

Figure 5.3. a) Number of structures as a function of the carbon number for $\mathrm{C}_{\mathrm{x}} \mathrm{H}_{2 \mathrm{x}-4}$. b) Number of structures as a function of the DBE number, and c) estimated computational time as a function of the DBE.

Another alternative to reduce the computational time is by introducing "chemical constraints" and filtering schemes to better reproduce PAH structural diversity. For 


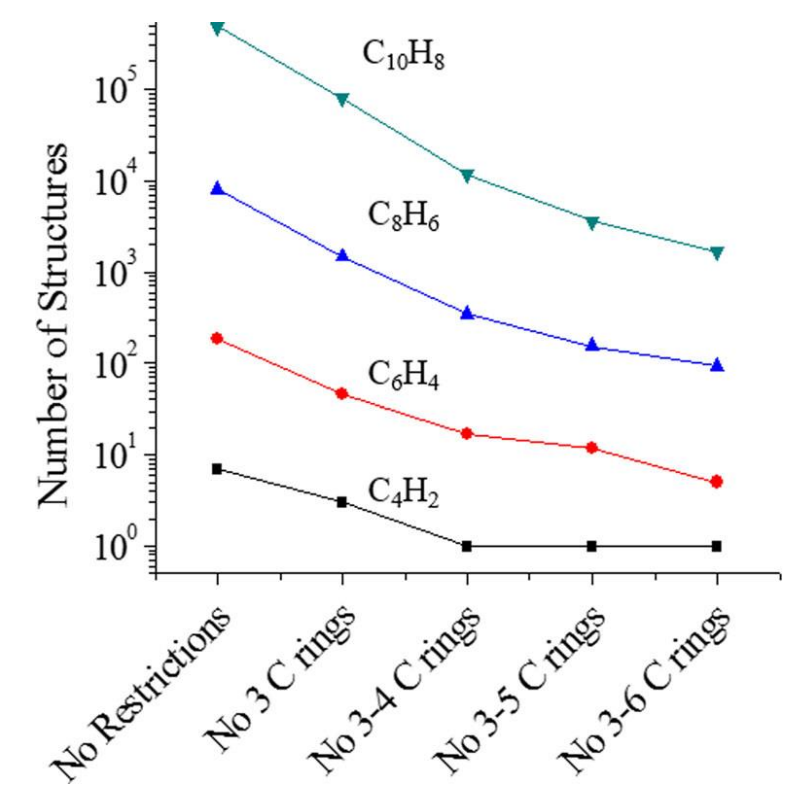

Figure 5.4. Number of potential structures dependence on the "chemical constraints".

example, one such scheme can be performed by introducing ring size constraints which will reduce the number of structures that are generated (see Fig. 5.4). The number of constraints typically depends on the type of molecules of interest. That is, for fossil fuel analysis the most common ring structures are napthenes and aromatics. ${ }^{43}$ This strategy can be further developed by incorporating structural constraints from IMS $/ \mathrm{MS}^{\mathrm{n}}$ and structural features from the chemical class of interest. For example, if the parent molecular ion can be isolated by $\mathrm{IMS}^{\mathrm{n}}$ and/or $\mathrm{MS}^{\mathrm{n}}$, the search algorithm will add constraints from the fragment ions, thus reducing the total number of candidate structures and the overall computational time. It should be noted, that structures for a given chemical formula only require to be calculated once; that is, a tridimensional database can be compiled and updated over time for the chemical class of interest. Another alternative to atomistic structural generation is the use of genetic algorithms, where structures are built according to set evolutionary rules. This has been shown in the case of small ionic cluster systems, ${ }^{44}$ drug discovery where structures were generated de-novo as a tool alongside high 
throughput screening, ${ }^{45,46}$ as well as in the case of NMR elucidation. ${ }^{47}$ Although genetic algorithms have not been used in the workflow proposed, their implementation can be included as part of the in silico structural generation.

\subsection{Conclusions}

A single analysis utilizing SA-TIMS-FTMS can provide a chemical formula and accurate mobility measurement from a complex mixture of fossil fuels without the need of sample pre-fractionation. Molecular structure elucidation from SA-TIMS-FTMS data typically requires the use of chemical standards and/or theoretical calculations of candidate structures. The workflow described here permits the generation of PAH structures by utilizing chemical databases and/or in silico structural generators, followed by geometry optimization and CCS calculations. Examples shown illustrate that this approach is feasible and that less than a $2 \%$ difference is observed between theoretical and experimental CCS. Moreover, potential computational challenges and alternatives are discussed to minimize the computation time and to increase the confidence in the structural assignment with increasing carbon number and DBE. For example, it was shown that the use of chemical constraints, such as limiting functional groups, or ring size, allows a reduction in the number of potential structures that are theoretically generated and their computational time. The proposed workflow can be adapted to different IMS scenarios, utilize different CCS calculators, and has the potential to include $M S^{\mathrm{n}}$ and $\mathrm{IMS}^{\mathrm{n}}$ measurements for faster and more accurate tridimensional structural assignment. 


\subsection{References}

1.Kanu AB, Hill HH Jr (2007) Identity confirmation of drugs and explosives in ion mobility spectrometry using a secondary drift gas. Talanta 73:692-699

2.Schenk ER, Mendez V, Landrum JT, Ridgeway ME, Park MA, Fernandez-Lima F (2014) Direct observation of differences of carotenoid polyene chain cis/trans isomers resulting from structural topology. Anal Chem 86:2019-2024

3.Pierson NA, Chen L, Russell DH, Clemmer DE (2013) Cis-trans isomerizations of proline residues are key to bradykinin conformations. J Am Chem Soc 135:3186-3192

4.Merenbloom SI, Glaskin RS, Henson ZB, Clemmer DE (2009) High-resolution ion cyclotron mobility spectrometry. Anal Chem 81:1482-1487

5.Sawyer HA, Marini JT, Stone EG, Ruotolo BT, Gillig KJ, Russell DH (2005) The structure of gas-phase bradykinin fragment 1-5 (RPPGF) ions: an ion mobility spectrometry and H/D exchange ion-molecule reaction chemistry study. J Am Soc Mass Spectrom 16:893-905

6.Schenk ER, Ridgeway ME, Park MA, Leng F, Fernandez-Lima F (2014) Isomerization kinetics of at hook decapeptide solution structures. Anal Chem 86:12101214

7.Molano-Arevalo JC, Hernandez DR, Gonzalez WG, Miksovska J, Ridgeway ME, Park MA, Fernandez-Lima F (2014) Flavin adenine dinucleotide structural motifs: from solution to gas phase. Anal Chem 86:10223-30

8.Ruotolo BT, Verbeck GF, Thomson LM, Woods AS, Gillig KJ, Russell DH (2002) Observation of conserved solution-phase secondary structure in gas-phase tryptic peptides. J Proteome Res 1:303

9.May JC, Goodwin CR, Lareau NM, Leaptrot KL, Morris CB, Kurulugama RT, Mordehai A, Klein C, Barry W, Darland E, Overney G, Imatani K, Stafford GC, Fjeldsted JC, McLean JA (2014) Conformational ordering of biomolecules in the gas phase: nitrogen collision cross sections measured on a prototype high resolution drift tube ion mobilitymass spectrometer. Anal Chem 86: 2107-2116

10. Gillig KJ, Russell DH (2001) A periodic field focusing ion mobility spectrometer. The Texas A \& M University System 36

11. Gillig KJ, Ruotolo BT, Stone EG, Russell DH (2004) An electrostatic focusing ion guide for ion mobility-mass spectrometry. Int J Mass Spectrom 239:43-49

12. Silveira JA, Gamage CM, Blase RC, Russell DH (2010) Gas-phase ion dynamics in a periodic-focusing DC ion guide. Int J Mass Spectrom 296:36-42 
13. Guo Y, Wang J, Javahery G, Thomson BA, Siu KWM (2004) Ion mobility spectrometer with radial collisional focusing. Anal Chem 77: 266-275

14. Koeniger SL, Merenbloom SI, Valentine SJ, Jarrold MF, Udseth HR, Smith RD, Clemmer DE (2006) An IMS-IMS analogue of MS-MS. Anal Chem 78:4161-4174

15. Kurulugama RT, Nachtigall FM, Lee S, Valentine SJ, Clemmer DE (2009) Overtone mobility spectrometry: Part 1. Experimental observations. J Am Soc Mass Spectrom 20:729-737

16. Glaskin RS, Valentine SJ, Clemmer DE (2010) A scanning frequency mode for ion cyclotron mobility spectrometry. Anal Chem 82: 8266-8271

17. Kolakowski BM, Mester Z (2007) Review of applications of high-field asymmetric waveform ion mobility spectrometry (FAIMS) and differential mobility spectrometry (DMS). Analyst 132: 842-864

18. Pringle SD, Giles K, Wildgoose JL, Williams JP, Slade SE, Thalassinos K, Bateman RH, Bowers MT, Scrivens JH (2007) An investigation of the mobility separation of some peptide and protein ions using a new hybrid quadrupole/travelling wave IMS/oaToF instrument. Int J Mass Spectrom 261:1-12

19. Bush MF, Hall Z, Giles K, Hoyes J, Robinson CV, Ruotolo BT (2010) Collision cross sections of proteins and their complexes: a calibration framework and database for gas-phase structural biology. Anal Chem 82: 9557-9565

20. Fernandez-Lima FA, Kaplan DA, Park MA (2011) Note: integration of trapped ion mobility spectrometry with mass spectrometry. Rev Sci Instrum 82:126106

21. Fernandez-Lima F, Kaplan D, Suetering J, Park M (2011) Gas-phase separation using a trapped ion mobility spectrometer. Int J Ion Mobility Spectrom 14: 93-98

22. Castellanos A, Benigni P, Hernandez D, DeBord J, Ridgeway M, Park M, Fernandez-Lima F (2014) Fast screening of polycyclic aromatic hydrocarbons using trapped ion mobility spectrometry-mass spectrometry. Anal Meth 6:9328-9332

23. Fernandez-Lima FA, Wei H, Gao YQ, Russell DH (2009) On the structure elucidation using IMS and Molecular Dynamics. J Phys Chem A 113: 8221-8234

24. Schenk E, Almeida R, Miksovska J, Ridgeway M, Park M, Fernandez-Lima F (2015) Kinetic intermediates of Holo- and apo-myoglobin studied using HDX-TIMS-MS and molecular dynamic simulations. J Am Soc Mass Spectrom 26:555-563

25. Schenk E, Nau F, Fernandez-Lima F (2015) Theoretical predictor for candidate structure assignment from IMS data of biomolecule-related conformational space. Int J Ion Mobility Spectrom 18: 1-7 
26. Benigni P, Thompson CJ, Ridgeway ME, Park MA, Fernandez-Lima F (2015) Targeted high-resolution ion mobility separation coupled to ultrahigh-resolution mass spectrometry of endocrine disruptors in complex mixtures. Anal Chem 87: 4321-5

27. Panda SK, Brockmann K-J, Benter T, Schrader W (2011) Atmospheric pressure laser ionization (APLI) coupled with Fourier transform ion cyclotron resonance mass spectrometry applied to petroleum samples analysis: comparison with electrospray ionization and atmospheric pressure photoionization methods. Rapid Comm Mass Spectrom 25:2317-2326

28. Panda SK, Andersson JT, Schrader W (2007) Mass-spectrometric analysis of complex volatile and nonvolatile crude oil components: a challenge. Anal Bioanal Chem 389:1329-1339

29. Schrader W, Panda SK, Brockmann KJ, Benter T (2008) Characterization of nonpolar aromatic hydrocarbons in crude oil using atmospheric pressure laser ionization and Fourier transform ion cyclotron resonance mass spectrometry (APLI FT-ICR MS). Analyst 133: 867-869

30. Gugisch R, Kerber A, Kohnert A, Laue R, Meringer M, Rücker C, Wassermann A (2012) MOLGEN 5.0, a molecular structure generator. Submitted to Bentham Science Publishers Ltd, 2013

31. Bangov IP (1996) Generation of molecular graphs based on flexible utilization of the available structural information. Discrete Appl Math 67:27-49

32. Stoyanov B, Petrov E, Kochev N, Bangov I (2014) A novel program for computer-aided generation of 2D chemical structures. Bulgarian Chem Comm 46:215.

33. Campuzano I, Bush MF, Robinson CV, Beaumont C, Richardson K, Kim H, Kim HI (2011) Structural characterization of drug-like compounds by ion mobility mass spectrometry: comparison of theoretical and experimentally derived nitrogen collision cross sections. Anal Chem 84: 1026-1033

34. Kim HI, Kim H, Pang ES, Ryu EK, Beegle LW, Loo JA, Goddard WA, Kanik I (2009) Structural characterization of unsaturated phosphatidylcholines using traveling wave ion mobility spectrometry. Anal Chem 81:8289

35. Larriba C, Hogan CJ (2013) Ion mobilities in diatomic gases: measurement versus prediction with non-specular scattering models. J Phys Chem A 117: 3887-3901

36. Larriba C, Hogan CJ Jr (2013) Free molecular collision cross section calculation methods for nanoparticles and complex ions with energy accommodation. $\mathrm{J}$ Comp Phys 251:344-363 
37. Ouyang H, Larriba-Andaluz C, Oberreit D, Hogan C Jr (2013) The collision cross sections of iodide salt cluster ions in air via differential mobility analysis-mass spectrometry. J Am Soc Mass Spectrom 24:1833-1847

38. Cho Y, Kim YH, Kim S (2011) Planar limit-assisted structural interpretation of saturates/aromatics/resins/asphaltenes fractionated crude oil compounds observed by fourier transform ion cyclotron resonance mass spectrometry. Anal chem 83:6068-6073

39. Hsu CS, Lobodin VV, Rodgers RP, McKenna AM, Marshall AG (2011) Compositional boundaries for fossil hydrocarbons. Energy \& Fuels, 25:2174-2178

40. Wise S, Poster D, Leigh S, Rimmer C, Mössner S, Schubert P, Sander L, Schantz M (2010) Polycyclic aromatic hydrocarbons (PAHs) in a coal tar standard reference material-SRM 1597a updated. Anal Bioanal Chem 398:717-728

41. Bouzzine SM, Bouzakraoui S, Bouachrine M, Hamidi M (2005) Density functional theory (B3LYP/6-31G*) study of oligothiophenes in their aromatic and polaronic states. J Mol Struc-Theochem 726:271-276

42. Förner W, Utz W (2002) Correlated ab initio and density functional calculations on small model molecules for the unit cell of polyparaphenylene in its aromatic and quinoidal forms: equilibrium geometries and vibrational spectra. J Mol Struc-Theochem 618:65-84

43. Silva SL, Silva AMS, Ribeiro JC, Martins FG, Da Silva FA, Silva CM (2011) Chromatographic and spectroscopic analysis of heavy crude oil mixtures with emphasis in nuclear magnetic resonance spectroscopy: a review. Analytica Chimica Acta 707:18-37

44. Fernandez-Lima FA, VilelaNeto OP, Pimentel AS, Ponciano CR, Pacheco MAC, Nascimento MAC, Silveira EFD (2009) A theoretical and experimental study of positive and neutral $\mathrm{LiF}$ clusters produced by fast ion impact on a polycrystalline $\mathrm{LiF}$ target. J Phys Chem A 113:1813-1821

45. Douguet D, Thoreau E, Grassy G (2000) A genetic algorithm for the automated generation of small organic molecules: drug design using an evolutionary algorithm. J Comput Aided Mol Des 14: 449-466

46. Schneider G, Fechner U (2005) Computer-based de novo design of drug-like molecules. Nat Rev Drug Discov 4:649-663

47. Han Y, Steinbeck C (2004) Evolutionary-algorithm-based strategy for computer-assisted structure elucidation. J Chem Inf Comp Sci 44:489-498 


\section{CHAPTER VI}

TARGETED HIGH-RESOLUTION ION MOBILITY SEPARATION COUPLED TO ULTRAHIGH-RESOLUTION MASS SPECTROMETRY OF ENDOCRINE DISRUPTORS IN COMPLEX MIXTURES

(Adapted with permission from Benigni et al., 2015, Analytical Chemistry, copyright 2017 American Chemical Society) 


\subsection{Abstract}

Traditional separation and detection of targeted compounds from complex mixtures from environmental matrices requires the use of lengthy prefractionation steps and highresolution mass analyzers due to the large number of chemical components and their large structural diversity (highly isomeric). In the present work, selected accumulation trapped ion mobility spectrometry (SA-TIMS) is coupled to Fourier transform ion cyclotron resonance mass spectrometry (FT-ICR MS) for direct separation and characterization of targeted endocrine-disrupting compounds (EDC) from a complex environmental matrix in a single analysis. In particular, targeted identification based on high-resolution mobility ( $R$ $\sim 70-120)$ and ultrahigh-resolution mass measurements $(\mathrm{R}>400,000)$ of seven commonly targeted EDC and their isobars (e.g., bisphenol A, (Z)- and (E)-diethylstilbestrol, hexestrol, estrone, $\alpha$-estradiol, and 17-ethynylestradiol) is shown from a complex mixture of watersoluble organic matter (e.g., Suwannee River Fulvic Acid Standard II) complemented with reference standard measurements and theoretical calculations ( $<3 \%$ error).

\subsection{Introduction}

The persistence, bioaccumulation and fate of a variety of chemical compounds has gained substantial interest in the scientific community due to their short and long-term effects on human and animal health (e.g., environmental contaminants such as pharmaceuticals, personal-care products, surfactants, perfluorinated and perchlorinated compounds, and many other chemical classes). Typical practice includes mitigation of their emission by the identification of the anthropogenic sources and by regular monitoring of their levels; however, these analyses can be analytically challenging due to the complexity 
of the sample matrix. For example, the study of endocrine-disrupting compounds (EDC) requires their identification from complex environmental and biological matrices using accurate analytical methods. ${ }^{1,2}$ Standard protocols involve the use of lengthy sample preparation, prefractionation steps, and chromatographic separations (e.g., derivitization prior to gas chromatography, and liquid chromatography) followed by mass spectrometry analysis (e.g., high-resolution TOF-MS or MS/MS). ${ }^{3-6}$ Alternatively, it has been shown that ultrahigh-resolution mass spectrometry (e.g., FT-ICR MS) is a powerful technique for the identification of targeted and nontargeted components using exact mass measurements, as well as for the elucidation of potential interferences. ${ }^{7-13}$

Over the last decades, with the advent of more versatile and higher resolution forms of ion mobility separations (e.g., periodic focusing DC ion guide, ${ }^{14-16}$ segmented quadrupole drift cell, ${ }^{17}$ multistage IMS,${ }^{18-20}$ field asymmetric IMS (FAIMS), ${ }^{21}$ traveling wave ion guide, ${ }^{22,23}$ and trapped ion mobility spectrometry $\left.{ }^{24-26}\right)$, progress toward the identification of molecules of interest embedded in complex matrices has been achieved by reducing the chemical noise and increasing the peak capacity and the dynamic range. ${ }^{27-}$ ${ }^{35}$ Complementary studies using high-resolution IMS-MS devices and ultrahigh-resolution MS analyzers have shown their unique advantages for the separation of structural and geometrical isomers and their chemical identification from exact mass measurements. ${ }^{36,37}$ More recently, the advantage of coupling some variants of IMS separations (e.g., FAIMS) to ultrahigh-resolution mass spectrometry has been shown for online separation of structural isomers. ${ }^{38-45}$ 
In the present work, we show for the first time the advantages of coupling selected accumulation trapped ion mobility spectrometry to an ultrahigh-resolution mass spectrometer for targeted analysis of EDC in a complex environmental matrix and the elucidation of potential interferences. Seven endocrine disruptors (bisphenol A, (Z)- and (E)-diethylstilbestrol, hexestrol, estrone, $\alpha$-estradiol, and 17- $\alpha$-ethynylestradiol) were analyzed in a complex mixture of water-soluble organic matter (e.g., Suwannee River Fulvic Acid Standard II). Identity of the targeted EDC compounds was confirmed with complementary measurements using reference standards and mobility values from theoretical calculations of candidate structures.

\subsection{Experimental Section}

Seven commonly targeted EDC and their isobars (e.g., bisphenol A, (Z)- and (E)diethylstilbestrol, hexestrol, estrone, $\alpha$-estradiol, and 17- $\alpha$-ethynylestradiol) were purchased from Sigma-Aldrich (St. Louis, MO) and used as received. As a complex mixture of water-soluble organic matter, a Suwannee River Fulvic Acid Standard II (SRFA) was obtained from the International Humic Substances Society (St. Paul, MN) and used as received. All solvents used in these studies were analytical grade or better and purchased from Fisher Scientific (Pittsburgh, PA). EDC were dissolved in 50/50 v/v methanol/water and added at $5 \mathrm{ppb}$ to a $20 \mu \mathrm{g} / \mathrm{mL}$ solution of the SRFA mixture. A Tuning Mix calibration standard (TuneMix, G24221A) was purchased from Agilent Technologies (Santa Clara, CA) and used as received. Details on the Tunemix structures (e.g., $m / z$ 322, $K_{0}=1.376 \mathrm{~cm}^{2} \mathrm{~V}^{-1} \mathrm{~s}^{-1}$ and $\mathrm{m} / z 622, K_{0}=1.013 \mathrm{~cm}^{2} \mathrm{~V}^{-1} \mathrm{~s}^{-1}$ ) can be found elsewhere. ${ }^{24,46}$ All experiments were performed in triplicate. 
Details regarding the TIMS operation and specifics compared to traditional IMS can be found elsewhere. ${ }^{24,25,47-49}$ Briefly, the TIMS mobility separation is based on holding the ions stationary using an electric field against a moving gas. In traditional TIMS operation, multiple geometric isomers/conformers are trapped simultaneously at different $E$ values resulting from a voltage gradient applied across the TIMS tunnel (more details in refs 47-49). The $E$ gradient defines the IMS range that is trapped and analyzed, thus allowing low-resolution (large $E$ gradient) and high-resolution (small $E$ gradient) IMS separations. The possibility to separate and accumulate single isomers/conformers over time in a TIMS device relies on selecting the $E$ gradient and by performing stepwise elutions into the mass analyzers by reducing the voltage range within a single trapping step. Multistep elutions are typically used when TIMS is coupled to fast-acquisition-rate MS analyzers (e.g., TOF-MS). However, when TIMS is coupled to slower MS analyzers (e.g., FT-ICR MS), TIMS's operation is changed to single-step elutions (from a small $E$ gradient that defines the IMS resolution) and sequential scanning of the $E$ gradient range. That is, each isomer/conformer eluting from the IMS cell can be described by a $E \pm \Delta E$ value; the smaller the $\Delta E$ value, the higher the IMS resolution and accuracy to determine the $K_{0} \pm \Delta$ $K_{0}$ value. This mode of operation is called selected accumulation trapped ion mobility spectrometry (SA-TIMS). SA-TIMS operation was controlled using in-house software, written in National Instruments Lab VIEW (2012, v. 12.0f3), and synchronized with the FT-ICR MS acquisition program. IMS separation was performed using nitrogen as a bath gas at ca. $300 \mathrm{~K}$, and the gas flow velocity was controlled by the pressure difference between entrance funnel $P_{1}=2.6 \mathrm{mbar}$, and the exit funnel $P_{2}=1.3 \mathrm{mbar}$. $P_{1}$ and $P_{2}$ values were held constant for all experiments. The same RF (2020 kHz and 240Vpp) was applied 
to all electrodes including the entrance funnel, the mobility separating section, and the exit funnel. An electrospray ionization source (Apollo II ESI design, Bruker Daltonics, Inc., MA) was used for all the analyses. The IMS cell was operated using a fill/trap/elute/quench sequence of 250-600/90/25/10 ms, using an average of 20 IMS scans per MS spectrum and a voltage difference across the $\Delta E$ gradient of $0.5-1.0 \mathrm{~V}$. Under these conditions, the average IMS resolution was 70-120. MS acquisition was optimized for highest transmission in the $m / z$ 200-600 in the 7T Solarix FT-ICR MS spectrometer (Bruker Daltonics Inc., MA). MS spectra were acquired at 1-16 Mword using half-sin apodization followed by fast-Fourier transform and broadband phase correction into absorption mode spectra with resolutions of $R \sim 75,000-730,000$ at $\mathrm{m} / z$ 400. External IMS and MS calibration was performed utilizing Agilent ESI-ToF tuning mix (Tunemix, G2421A, Agilent Technologies, Santa Clara, CA).

Candidate structures were proposed for each molecular ion of interest observed in the IMS-FT-ICR MS experiments. ${ }^{50}$ Theoretical ion-neutral collision cross sections were calculated using MOBCAL version for nitrogen ${ }^{51,52}$ and IMoS (v 1.04b) $)^{53-55}$ software with a bath gas at ca. $300 \mathrm{~K}$. In the IMoS calculations, 100 total rotations were performed using the diffuse hard sphere scattering method with a Maxwell distribution. Partial atomic charges were calculated using the Merz-Singh-Kollman scheme constrained to the molecular dipole moment. ${ }^{56,57}$ All optimized geometries and partial atomic charges are provided in Appendix 6.3. 


\subsection{Results and Discussion}

One of the main analytical challenges during targeted analysis in complex samples is the presence of isobaric and isomeric interferences. Ultrahigh-resolution MS analysis will routinely detect multiple molecular components at the level of nominal mass during the analysis of complex mixtures. For example, the FT-ICR MS analysis of EDC compounds from a complex mixture will typically yield 8-10 peaks per nominal mass (see Figure 6.1). Closer inspection of the FT-ICR MS data shows that chemical formulas for the EDC compounds (see Table 6.1) and the SRFA components (see Appendix 6) can be
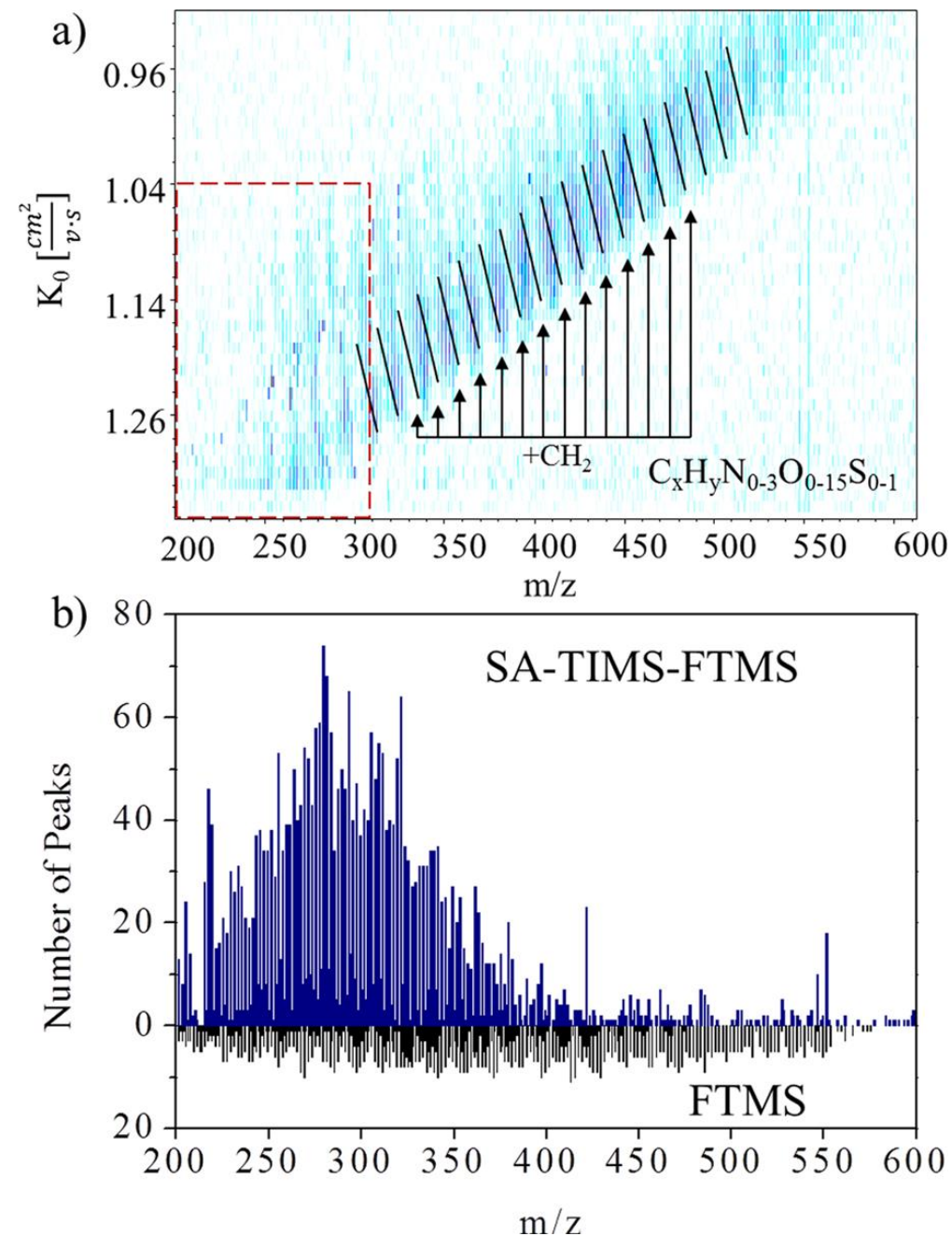

Figure 6.1. (a) 2D-IMS-FTMS contour plot of the complex mixture containing EDC compounds (dashed square) and SRFA standard. (b) Number of peaks identified at the nominal mass in SA-TIMS-FTMS and FTMS analysis. 
assigned with sub ppm accuracy. When FT-ICR MS analysis is complemented with orthogonal separations SA-TIMS-FTMS, an increase in peak capacity of 2-10-fold is observed per nominal mass; that is, multiple molecular signatures are observed in the $2 \mathrm{D}$ IMS-FTMS plot per mass signal. This result is a consequence of the structural diversity and complexity of the sample. For example, molecular compounds from the environmental matrix (SRFA standard) can be described by the generic formula $\mathrm{C}_{x} \mathrm{H}_{y} \mathrm{~N}_{0-3} \mathrm{O}_{0-15} \mathrm{~S}_{0-1}$, where $75 \%$ are highly conjugated oxygen containing compounds $\left(\mathrm{O}_{1}-\mathrm{O}_{15}\right.$, see Appendix 6.1$) .^{7-13}$ Inspection of the double bond equivalents (DBE) as a function of the carbon number for the oxygen containing series $\left(\mathrm{C}_{x} \mathrm{H}_{y} \mathrm{O}_{1-15}\right)$ showed the large structural diversity expected from fulvic acids (see Appendix 6.2)

Closer inspection to the IMS-FTMS data shows that separation and identification of the EDC targeted compounds from other interferences was achieved (see Figure 6.2a). In particular, complementary analysis using reference standards of the EDC compounds permitted the molecular confirmation by exact mass (sub ppm) and by mobility $(<3 \%)$. In the example shown, two types of interferences were considered: (i) isobaric interferences between the EDC compound and the SRFA matrix (e.g., bisphenol A, $\alpha$-estradiol, and 17$\alpha$-ethynylestradiol), and (ii) simultaneous isobaric and isomeric between two EDC compounds and the SRFA matrix (e.g., estrone and hexestrol and (E)- and (Z)diethylstilbestrol). The high resolution of the SAIMS $\left(R_{\mathrm{IMS}} \sim 70-120\right)$ permitted baseline separation in both scenarios (see Figure 6.2b). For example, inspection of the IMS projection of the structural isomers estrone and hexestrol $\left(\mathrm{C}_{18} \mathrm{H}_{22} \mathrm{O}_{2}\right.$, error: $\left.0.09 \mathrm{ppm}\right)$ showed two baseline-resolved peaks at $K_{0}=1.215$ and $1.191 \mathrm{~cm}^{2} \mathrm{~V}^{-1} \mathrm{~s}^{-1}$, respectively. 


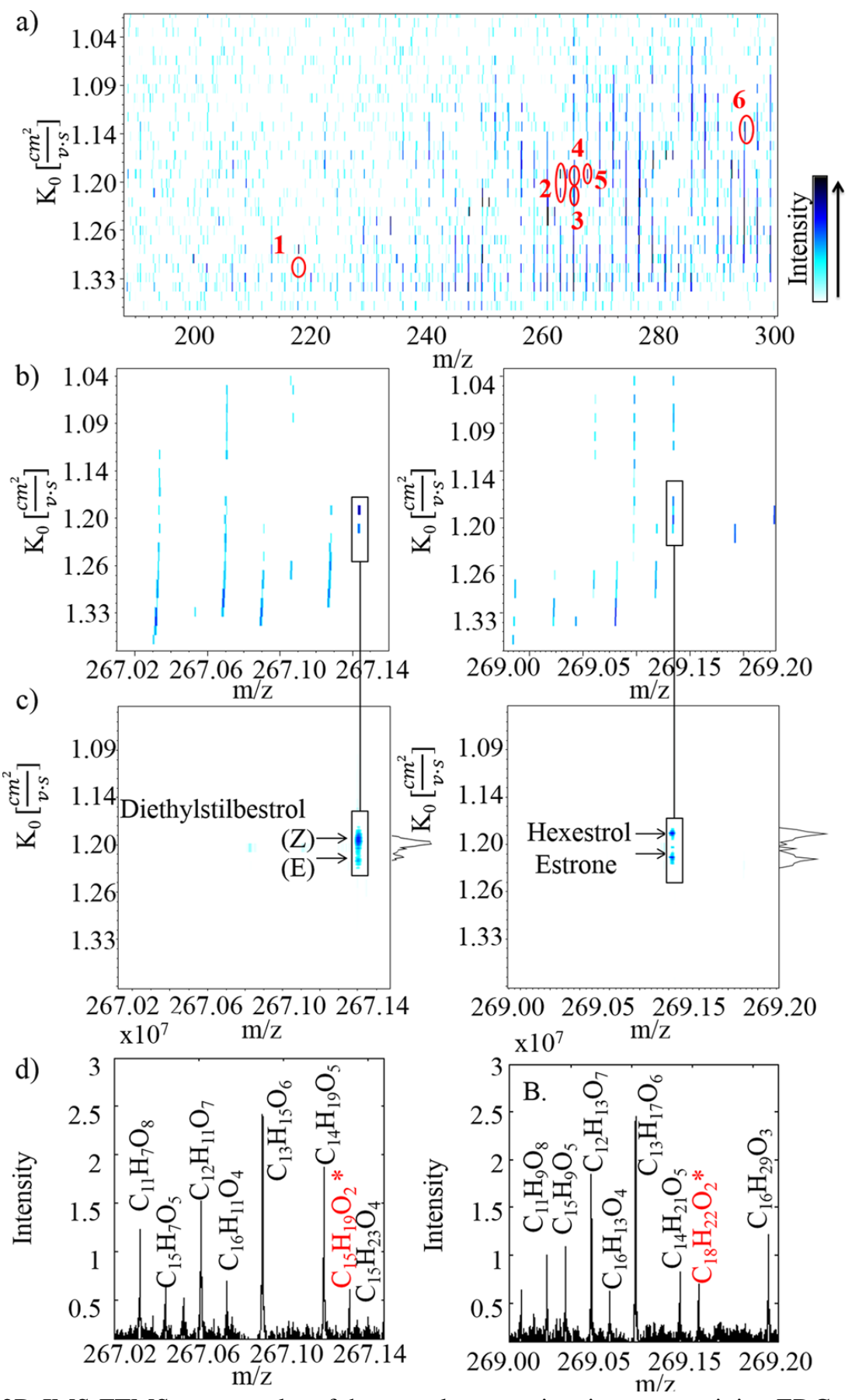

Figure 6.2. (a) 2D-IMS-FTMS contour plot of the complex organic mixture containing EDC compounds and SRFA standard. Notice the separation of (1) bisphenol A, (2) diethylstilbestrol, (3) estrone, (4) hexestrol, (5) $\alpha$-estradiol, (6) 17- $\alpha$-ethynylestradiol from the SRFA matrix. 2D-IMS-FTMS contour plot at the nominal mass for the structural isomers of (E)- and (Z)-diethylstilbestrol (left) and estrone and hexestrol (right) in (b) the complex sample, (c) the reference standards, and (d) the respective FTMS projections (* denotes the EDC formula).

Analogously, IMS projection of diethylstilbestrol (E) and (Z) structural isomers $\left(\mathrm{C}_{15} \mathrm{H}_{19} \mathrm{O}_{2}\right.$,

error: $0.25 \mathrm{ppm}$ ) showed two baseline-resolved peaks corresponding to the two cis/trans 
Table 6.1 Experimental SA-TIMS-FTMS and Theoretical Mass and Mobility Values for Bisphenol A, Diethylstilbestrol, Estrone, Hexestrol, $\alpha$-Estradiol, and 17- $\alpha$-Ethynylestradiol

\begin{tabular}{|c|c|c|c|c|c|c|c|c|}
\hline \multicolumn{7}{|c|}{ experimental } & \multicolumn{2}{|c|}{ theoretical } \\
\hline $\operatorname{exptl} m / z$ & ion formula & $\begin{array}{l}\text { theoretical } \\
\text { mass }\end{array}$ & $\begin{array}{l}\text { error } \\
(\mathrm{ppm})\end{array}$ & name & $\mathrm{K}_{0}\left[\mathrm{~cm}^{2} / \mathrm{v} \cdot \mathrm{s}\right]$ & $\begin{array}{l}\mathrm{CCS} \\
{\left[\mathrm{A}^{2}\right]}\end{array}$ & $\begin{array}{c}\text { MOBCAL TM CCS } \\
{\left[\mathrm{A}^{2}\right]}\end{array}$ & $\begin{array}{c}\text { IMoS DHSS CCS } \\
{\left[\mathrm{A}^{2}\right]}\end{array}$ \\
\hline 227.10766 & $\mathrm{C}_{15} \mathrm{H}_{15} \mathrm{O}_{2}$ & 227.10775 & -0.39 & bisphenol A & 1.305 & 162 & 161 & 161 \\
\hline 267.13924 & $\mathrm{C}_{18} \mathrm{H}_{19} \mathrm{O}_{2}$ & 267.13905 & -0.25 & diethylstilbestrol & 1.227 & 171 & $172(E)$ & $172(E)$ \\
\hline & & & & & 1.203 & 175 & $177(Z)$ & $175(Z)$ \\
\hline 269.15484 & $\mathrm{C}_{18} \mathrm{H}_{21} \mathrm{O}_{2}$ & 269.15470 & 0.09 & estrone & 1.215 & 174 & 169 & 172 \\
\hline 269.15484 & $\mathrm{C}_{18} \mathrm{H}_{21} \mathrm{O}_{2}$ & 269.15470 & 0.09 & hexestrol & 1.191 & 177 & 177 & 180 \\
\hline 271.17037 & $\mathrm{C}_{18} \mathrm{H}_{23} \mathrm{O}_{2}$ & 271.17035 & -0.05 & $\alpha$-estradiol & 1.203 & 175 & 177 & 176 \\
\hline 295.17011 & $\mathrm{C}_{20} \mathrm{H}_{23} \mathrm{O}_{2}$ & 295.17035 & 0.82 & $17-\alpha$-ethynylestradiol & 1.152 & 182 & 183 & 182 \\
\hline
\end{tabular}

isomers with $K_{0}=1.227$ and $1.203 \mathrm{~cm}^{2} \mathrm{~V}^{-1} \mathrm{~s}^{-1}$, respectively. Biological activity for

endocrine disruptors varies depending on the structural isomers which can be separated. ${ }^{58}$, 59

Beside the high mobility resolution that can be achieved using SA-TIMS, which allows direct identification with reference materials, it is a method of operation that also allows for direct mobility measurements. ${ }^{24,25,47-49}$ This translates into the additional potential for targeted EDC molecular assignment based on mobility values of candidate structures (see Table 6.1 and details on the EDC candidate structures in appendix 6.3). Comparison of experimental and theoretical mobility values for the targeted EDC compounds showed a good agreement ( $<3 \%$ error). Both theoretical CCS calculators yielded similar results and in good correspondence with the experimental trends. This alternative approach increases the practical value of SA-TIMS-FT-ICR MS when reference standards are not available.

\subsection{Conclusion}

The use of a novel variant SA-TIMS-FTMS for complementary, high-resolution mobility and ultrahigh-resolution mass separations is illustrated for targeted analysis of endocrine disrupting chemicals in a complex matrix. It was shown that targeted 
identification can be achieved based on accurate mobility and exact mass measurements and complemented with reference standard measurements and/or theoretical calculations. In particular, SA-TIMS high mobility resolution $\left(R_{\mathrm{IMS}} \sim 75-120\right)$ allowed the separation of chemical interferences from the sample matrix as well as the separation of EDC structural isomers. The use of theoretical calculations may significantly reduce the cost of targeted EDC analysis and permits the assignment of molecular structures with a high degree of confidence ( $<3 \%$ error).

\subsection{References}

1.Dwivedi, P.; Schultz, A. J.; H. H, H., Jr. Int. J. Mass Spectrom. 2010, 298, 78

2.Wyttenbach, T.; Batka, J. J.; Gidden, J.; Bowers, M. T. Int. J. Mass Spectrom. $1999,193,143$

94

3.Busico, F.; Moretti, G.; Cartoni, G. P.; Rosati, F. J. High Res. Chromat. 1992, 15,

4.Casademont, G.; Pérez, B.; García Regueiro, J. A. J. Chromat. B 1996, 686, 189

5.Daeseleire, E.; Vandeputte, R.; Van Peteghem, C. Analyst 1998, 123, 2595

6.Huang, C.-H.; Sedlak, D. L. Environ. Toxicol. Chem. 2001, 20, 133

7.Witt, M.; Fuchser, J.; Koch, B. P. Anal. Chem. 2009, 81, 2688

8.Koch, B. P.; Ludwichowski, K.-U.; Kattner, G.; Dittmar, T.; Witt, M. Marine Chemistry 2008, 111, 233

9.Rostad, C. E.; Leenheer, J. A. Anal. Chim. Acta 2004, 523, 269

10. Kujawinski, E. B.; Hatcher, P. G.; Freitas, M. A. Anal. Chem. 2001, 74, 413

11. Brown, T. L.; Rice, J. A. Anal. Chem. 1999, 72, 384

12. Fievre, A.; Solouki, T.; Marshall, A. G.; Cooper, W. T. Energy Fuels 1997, 11, 554

13. Stenson, A. C.; Marshall, A. G.; Cooper, W. T. Anal. Chem. 2003, 75, 1275 
14. Gillig, K. J.; Russell, D. H. The Texas A \& M University System: College Station, TX; Patent No. WO0165589, September 7, 2001; p 36.

15. Gillig, K. J.; Ruotolo, B. T.; Stone, E. G.; Russell, D. H. Int. J. Mass Spectrom. 2004, 239, 43

16. Silveira, J. A.; Gamage, C. M.; Blase, R. C.; Russell, D. H. Int. J. Mass Spectrom. 2010, 296, 36

17. Guo, Y.; Wang, J.; Javahery, G.; Thomson, B. A.; Siu, K. W. M. Anal. Chem. 2004, 77, 266

18. Koeniger, S. L.; Merenbloom, S. I.; Valentine, S. J.; Jarrold, M. F.; Udseth, H. R.; Smith, R. D.; Clemmer, D. E. Anal. Chem. 2006, 78, 4161

19. Kurulugama, R. T.; Nachtigall, F. M.; Lee, S.; Valentine, S. J.; Clemmer, D. E. J. Am. Soc. Mass Spectrom. 2009, 20, 729

20. Glaskin, R. S.; Valentine, S. J.; Clemmer, D. E. Anal. Chem. 2010, 82, 8266

21. Kolakowski, B. M.; Mester, Z. Analyst 2007, 132, 842

22. Pringle, S. D.; Giles, K.; Wildgoose, J. L.; Williams, J. P.; Slade, S. E.; Thalassinos, K.; Bateman, R. H.; Bowers, M. T.; Scrivens, J. H. Int. J. Mass Spectrom. 2007, 261, 1

23. Bush, M. F.; Hall, Z.; Giles, K.; Hoyes, J.; Robinson, C. V.; Ruotolo, B. T. Anal. Chem. 2010, 82, 9557

24. Hernandez, D. R.; DeBord, J. D.; Ridgeway, M. E.; Kaplan, D. A.; Park, M. A.; Fernandez-Lima, F. Analyst 2014, 139, 1913

25. Fernandez-Lima, F. A.; Kaplan, D. A.; Park, M. A. Rev. Sci. Instrum. 2011, 82, 126106

26. Fernandez-Lima, F.; Kaplan, D.; Suetering, J.; Park, M. Int. J. Ion Mobil. Spec. $2011,14,93$

27. Dugourd, P.; Hudgins, R. R.; Clemmer, D. E.; Jarrold, M. F. Rev. Sci. Instrum. $1997,68,1122$

28. Merenbloom, S. I.; Glaskin, R. S.; Henson, Z. B.; Clemmer, D. E. Anal. Chem. $2009,81,1482$

29. Kemper, P. R.; Dupuis, N. F.; Bowers, M. T. Int. J. Mass Spectrom. 2009, 287, 46 
30. Blase, R. C.; Silveira, J. A.; Gillig, K. J.; Gamage, C. M.; Russell, D. H. Int. J. Mass Spectrom. 2011, 301, 166

31. May, J.; Russell, D. J. Am. Soc. Mass Spectrom. 2011, 22, 1134

32. Kemper, P. R.; Bowers, M. T. J. Am. Soc. Mass Spectrom. 1990, 1, 197

33. Wu, C.; Siems, W. F.; Asbury, G. R.; Hill, H. H. Anal. Chem. 1998, 70, 4929

34. Liu, Y.; Clemmer, D. E. Anal. Chem. 1997, 69, 2504

35. Jarrold, M. F.; Constant, V. A. Phys. Rev. Lett. 1991, 67, 2994

36. Fernandez-Lima, F. A.; Becker, C.; McKenna, A. M.; Rodgers, R. P.; Marshall, A. G.; Russell, D. H. Anal. Chem. 2009, 81, 9941

37. Fasciotti, M.; Lalli, P. M.; Klitzke, C. F.; Corilo, Y. E.; Pudenzi, M. A.; Pereira, R. C. L.; Bastos, W.; Daroda, R. J.; Eberlin, M. N. Energy Fuels 2013, 27, 7277

38. Robinson, E. W.; Williams, E. R. J. Am. Soc. Mass Spectrom. 2005, 16, 1427

39. Robinson, E. W.; Garcia, D. E.; Leib, R. D.; Williams, E. R. Anal. Chem. 2006, 78,2190

40. Robinson, E. W.; Leib, R. D.; Williams, E. R. J. Am. Soc. Mass Spectrom. 2006, 17, 1470 259,87

41. Robinson, E. W.; Sellon, R. E.; Williams, E. R. Int. J. Mass Spectrom. 2007,

42. Saba, J.; Bonneil, E.; Pomiès, C.; Eng, K.; Thibault, P. J. Prot. Res. 2009, 8, 3355

43. Xuan, Y.; Creese, A. J.; Horner, J. A.; Cooper, H. J. Rapid Commun. Mass Spectrom. 2009, 23, 1963

44. Bridon, G.; Bonneil, E.; Muratore-Schroeder, T.; Caron-Lizotte, O.; Thibault, P. J. Prot. Res. 2011, 11, 927

45. Schrader, W.; Xuan, Y.; Gaspar, A. European J. Mass Spectrom. 2014, 20, 43

46. Flanagan, L. A. Hewlett-Packard Company: Palo Alto, CA; U.S. Patent No. 5872357 A, February 16, 1999; p 19.

47. Fernandez-Lima, F. A.; Kaplan, D. A.; Suetering, J.; Park, M. A. Int. J. Ion Mobil. Spec. 2011, 14, 93 
48. Schenk, E. R.; Ridgeway, M. E.; Park, M. A.; Leng, F.; Fernandez-Lima, F. Anal. Chem. 2014, 86, 1210

49. Schenk, E. R.; Mendez, V.; Landrum, J. T.; Ridgeway, M. E.; Park, M. A.; Fernandez-Lima, F. Anal. Chem. 2014, 86, 2019

50. Fernandez-Lima, F. A.; Wei, H.; Gao, Y. Q.; Russell, D. H. J. Phys. Chem. A $2009,113,8221$

51. Campuzano, I.; Bush, M. F.; Robinson, C. V.; Beaumont, C.; Richardson, K.; Kim, H.; Kim, H. I. Anal. Chem. 2011, 84, 1026

52. Kim, H. I.; Kim, H.; Pang, E. S.; Ryu, E. K.; Beegle, L. W.; Loo, J. A.; Goddard, W. A.; Kanik, I. Anal. Chem. 2009, 81, 8289

53. Larriba, C.; Hogan, C. J. J. Phys. Chem. A 2013, 117, 3887

54. Larriba, C.; Hogan, C. J., Jr. J. Comput. Phys. 2013, 251, 344

55. Ouyang, H.; Larriba-Andaluz, C.; Oberreit, D.; Hogan, C., Jr. J. Am. Soc. Mass Spectrom. 2013, 24, 1833

56. Singh, U. C.; Kollman, P. A. J. Comput. Chem. 1984, 5, 129

57. Besler, B. H.; Merz, K. M.; Kollman, P. A. J. Comput. Chem. 1990, 11, 431

58. Winkler, V. W.; Nyman, M. A.; Egan, R. S. Steroids 1971, 17, 197

59. Katzenellenbogen, J. A.; Carlson, K. E.; Katzenellenbogen, B. S. J. Steroid Biochem. 1985, 22, 589 


\section{CHAPTER VII}

OVERSAMPLING SELECTIVE ACCUMULATION TRAPPED ION MOBILITY SPECTROMETRY COUPLED TO FT-ICR MS: FUNDAMENTALS AND APPLICATIONS

(Adapted with permission from Benigni et al., 2016, Analytical Chemistry, copyright 2017 American Chemical Society) 


\subsection{Abstract}

In the present paper, we describe the fundamentals and analytical advantages of Oversampling Selective Accumulation Trapped Ion Mobility Spectrometry (OSA-TIMS) when coupled to ultrahigh resolution mass analyzers (e.g., FT-ICR MS). During TIMS analysis, ion packages are spatially resolved based on their mobilities along the TIMS analyzer axis and multiple strategies can be utilized during the trapping and elution of the ion population of interest. In the case of OSA-TIMS-FT-ICR MS, the TIMS operation sequence, trapping conditions, and operations are optimized to increase the signal-to-noise and the number of points across the mobility domain, which leads to more accurate mobility and mass measurements. Experimental results show that accurate ion-neutral collision cross sections $(<1 \%)$ can be measured using OSA-TIMS-FT-ICR MS with high mobility resolving powers ( $R_{\mathrm{IMS}}$ up to 250$)$, high mass accuracy ( $\left.<1 \mathrm{ppm}\right)$, and ultrahigh mass resolution ( $R_{\mathrm{MS}}$ up to $600-1200 \mathrm{k}$ at $m / z$ 400) in a single analysis. The analytical advantages of OSA-TIMS over SA-TIMS were illustrated for the analysis of structural peptide isomers (SDGRG and GRGDS $[\mathrm{M}+\mathrm{H}]^{+}$), conformational isomers (AT-hook peptide 3 KRGRGRPRK $\left.[\mathrm{M}+2 \mathrm{H}]^{+2}\right)$, and a complex mixture of polyaromatic hydrocarbons (PAH) from coal tar. Baseline separation of the structural peptide isomers SDGRG and GRGDS, $[\mathrm{M}+\mathrm{H}]^{+}$, was observed, and three conformations were identified for the AT-hook peptide 3 KRGRGRPRK $[\mathrm{M}+2 \mathrm{H}]^{+2}$ during OSA-TIMS-FT-ICR MS. A 2fold increase in the number of molecular features and a 2-6-fold signal-to-noise increase was observed for OSA-TIMS when compared with SA-TIMS during the PAH analysis. This work provides the proof-of-principle for further application of OSA-TIMS-FT-ICR 
MS for the unsupervised analysis of complex mixtures based on the characterization of the conformational space and the assignment of chemical formulas in a single analysis.

\subsection{Introduction}

Typical operation of ultrahigh resolution mass spectrometers involves the storage of ions prior to analysis. In order to achieve very high sensitivity and resolution during FTICR MS measurements, the time-dependent ion signal can be measured for a very long time (tens to hundreds of seconds), thus, allowing for precise determination of the ion cyclotron frequencies. ${ }^{1,2}$ With the advent of new generation ICR cell designs, ${ }^{3-19}$ higher field magnets, ${ }^{20,21}$ and processing modes (e.g., absorption mode), ${ }^{22-27}$ the characterization of complex mixtures in a single analysis using FT-ICR MS is increasingly becoming the method of choice over shorter analysis time. The loss of coherence (dephasing) of the ion package during FT-ICR MS leads to dampening or decrease of the signal of interest, thus, limiting the sensitivity and resolution. ${ }^{28-31}$ This loss is typically associated with ion collisions with background molecules, inhomogenieties in the magnetic and electric field, and coalescence of the ion signal due to Coulombic interactions between the ions. ${ }^{32-35}$ The Coulombic interaction can also reduce the number of ions that can be effectively trapped and measured (space charge effects), thus, limiting the dynamic range and sensitivity in the analysis of complex mixtures. Previous work has shown alternatives to reduce the space charge effects in the ICR cell by changing the duration of the ionization event, ${ }^{36}$ preselection of the ions of interest using a mass analyzer, ${ }^{37,} 38$ ejection of high abundance species,${ }^{38-40}$ or selectively accumulating ions directly in the FT-ICR cell. ${ }^{41}$ Ion selection has also been performed within the FT-ICR cell utilizing phase selective excitation-de- 
excitation pulses, termed 2D-FT-ICR MS, in order to select and fragment target molecules by infrared multiphoton dissociation (IRMPD) or electron capture dissociation (ECD) $)^{42-47}$ or blackbody infrared radiation dissociation (BIRD). ${ }^{48-51}$ Alternatively, the use of gas and liquid chromatography, as well as the choice of the ionization source, has also shown an increase in sensitivity and dynamic range while typically increasing the total analyst time to tens of minutes..$^{52-55}$

In a different approach to effectively reduce the space charge effects, scanning and time-dispersive ion mobility spectrometry methods have been successfully coupled to FTICR MS. ${ }^{56-63}$ In particular, a low pressure drift tube based IMS (DT-IMS) was coupled to FT-ICR MS to study ion-molecule reaction chemistry. ${ }^{62}$ High-field asymmetric waveform ion mobility spectrometry (FA-IMS) has also been used with FT-ICR MS resulting in increased sensitivity, lower detection limits, and increased dynamic range for the analysis of poly(ethylene glycol), ${ }^{60}$ proteins,,${ }^{57-59}$ and glycans ${ }^{56}$ as a consequence of a reduction of the adverse Coulombic effects by mobility-selective injection in the FT-ICR MS. Atmospheric pressure drift tube based IMS (DT-IMS) was also successfully coupled to FTICR MS using a dual gate system for the separation of isomeric phosphopeptides and measurements of reduced mobility constants. ${ }^{61}$ In a recent report, we described the use of selective accumulation trapped ion mobility spectrometry (SA-TIMS) coupled to FT-ICR MS for targeted analysis of compounds of interest in complex mixtures using accurate reduced mobility $\left(R_{\mathrm{IMS}}=70-120\right)$ and mass measurements $(<10 \mathrm{ppb})$ for the case of endocrine disruptors in a complex environmental matrix. ${ }^{63}$ The SA-TIMS operation is based on the trapping of a mobility range prior to injection into the FT-ICR MS; scanning 
of the mobility range allows for the generation of the 2D-IMS-MS plots of complex mixtures and the performance of mobility separated FT-ICR MS/MS experiments. ${ }^{63,64}$ While SA-TIMS-FT-ICR MS presented multiple advantages over previously reported IMS approaches, its analytical application for unsupervised molecular feature identification was mostly limited to the search for molecular formulas in the MS domain with limited identification in the IMS domain due to the number of points per mobility band (e.g., typically few points across a peak in an IMS band using a high mobility resolution scan mode). ${ }^{63,65}$

In the present work, we describe for the first time the use of oversampling SATIMS (OSA-TIMS) coupled to FT-ICR MS. The OSA-TIMS fundamental mode of operation and their analytical application for the detection of molecular features in the IMS and MS domain are shown, as well as their advantages over previously described IMS FTICR MS methods. We also describe the advantages for the study of structural and conformational biomolecular isomers and the analysis of a complex mixture using unsupervised molecular feature detection. In particular, the advantages on mobility resolving power, signal-to-noise and number of molecular features determine from a 2DIMS-MS plot are illustrated with experimental data.

\subsection{Experimental Section}

\subsubsection{Materials and Reagents}

A Tuning Mix calibration standard (G24221A) was obtained from Agilent Technologies (Santa Clara, CA) and used as received. The SDGRG and GRGDS and KRGRGRPRK (AT-Hook peptide 3, ATHP3) peptides were purchased from Sigma- 
Aldrich (St. Louis, MO) and Advanced ChemTech Inc. (Louisville, KY), respectively. All peptide standards were received as a lyophilized powder and reconstituted in $10 \mathrm{mM}$ ammonium acetate in water to a final concentration of $10 \mu \mathrm{M}$. A standard reference material of polycyclic aromatic hydrocarbons (PAHs) in coal tar (SRM 1597a) was purchased from the National Institute of Standards and Technology (Baltimore, MA) and was diluted 1:100 in 50:50 v/v methanol/toluene. All solvents and ammonium salts utilized in this study were analytical grade or better and purchased from Fisher Scientific (Pittsburgh, PA).

a)
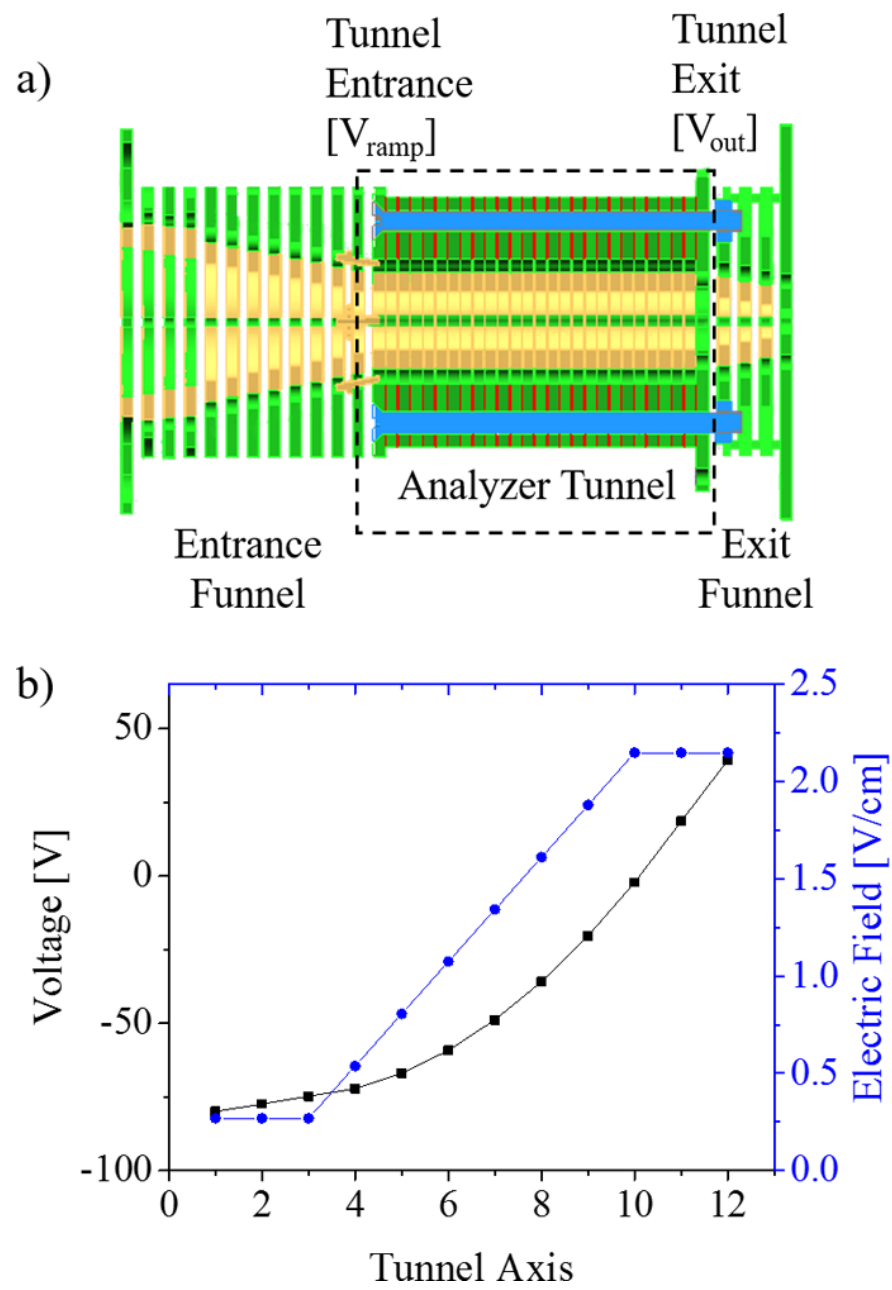

Figure 7.1. (a) Schematics of the TIMS cell and (b) profile of the voltage (black) and electric field (blue) across a simplified representation of the electrodes in the TIMS analyzer. 


\subsubsection{Instrumentation}

For this study, an orthogonal custom-built nano electrospray ionization (nanoESI) source based on a pulled glass capillary was placed on the front of a single bore, resistively coated glass capillary allowing aerodynamic focusing and desolvation of ions prior injection into the TIMS analyzer. The coal tar analysis was performed with an atmospheric pressure photo ionization source (APPI) based on the Apollo II design (Bruker Daltonics Inc., MA) using a Kr lamp with main emission bands at 10.0 and $10.6 \mathrm{eV}$. The TIMS-FTICR MS instrumentation has been previously described (see details for the TIMS in Figure 7.1a). ${ }^{63}$ Briefly, the instrument is a custom-built TIMS-FT-ICR MS prototype which incorporates a TIMS analyzer that substitutes the dual funnel entrance section of a 7T Solarix FT-ICR MS spectrometer equipped with an infinity ICR cell (Bruker Daltonics Inc., MA). The TIMS cartridge is comprised of three main regions: the entrance funnel, the mobility analyzer section (tunnel), and the exit funnel, with the same RF (840 kHz and 240-280 Vpp) applied to all electrodes. ${ }^{66-68}$ Each electrode is divided into four electrically insulated segments, which are used to create a dipole field in the entrance and exit section to focus the ions downstream, and a quadrupolar field in the separation region to radially confine the ions during the mobility selected ion trapping. The electrodes are electrically connected through a resistive divider in order to define the electric field across the TIMS analyzer axis. 

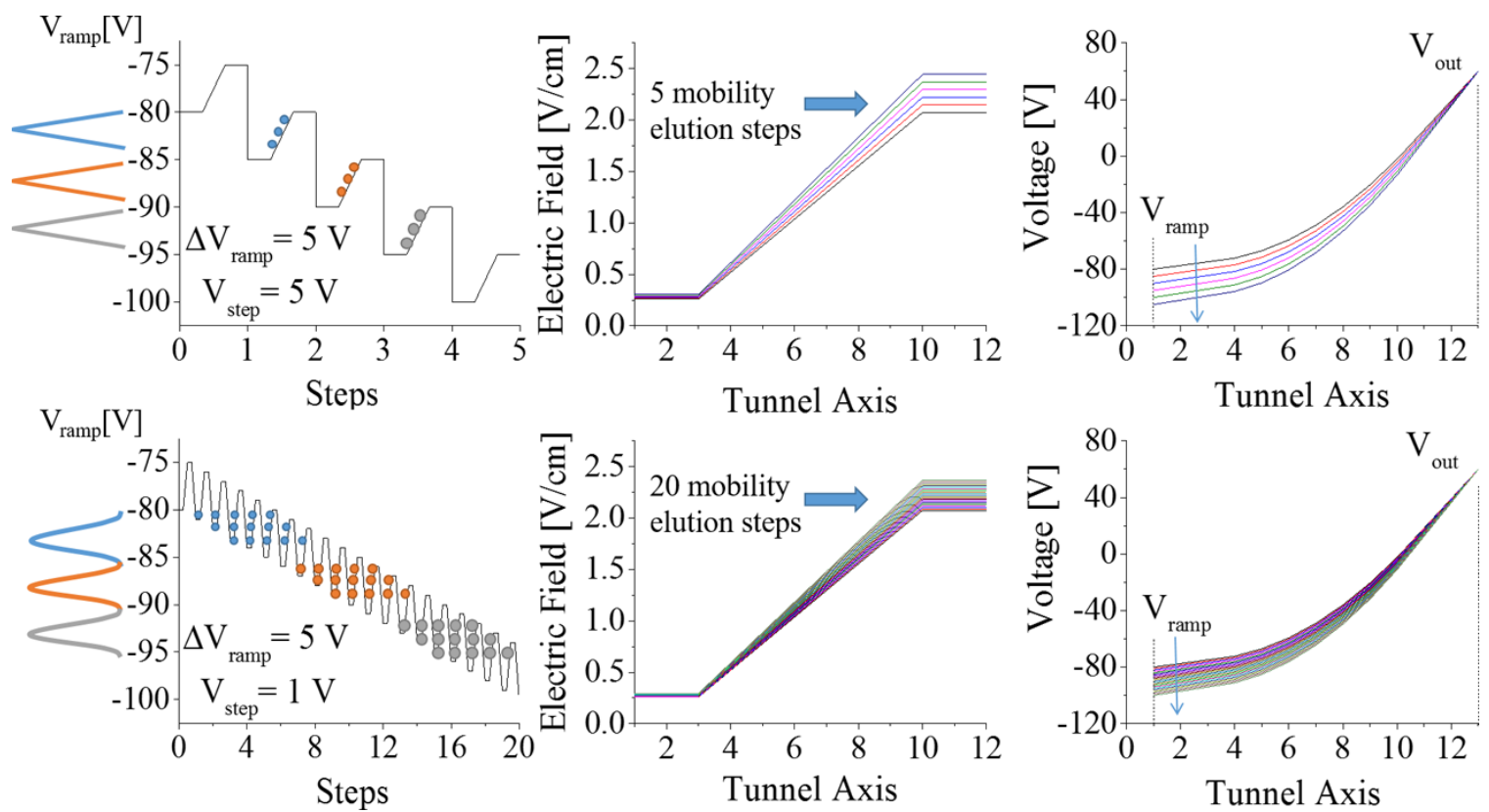

Figure 7.2. Comparison of the analysis sequence, electric field, and voltage applied in the TIMS analyzer during SA-TI.MS (top) and OSA-TIMS (bottom) for the analysis of the same mobility range using 5 elution steps and 20 elution steps. Notice that in SA-TIMS $\Delta$ Vramp $=$ Vstep and in OSA-TIMS $\Delta$ Vramp > Vstep.

\subsubsection{SA-TIMS versus OSA-TIMS Operation}

The concept behind TIMS is the use of an electric field to hold ions stationary against a moving gas, so that the drag force is compensated by the electric field and ion packages are separated across the TIMS analyzer axis based on their mobility. Mobility selection is defined by the electric field range applied in the analyzer region; the $V_{\text {out }}$ is typically held constant $\left(V_{\text {out }}=35 \mathrm{~V}\right)$, while the $V_{\text {ramp }}$ defines the mobility range that is trapped into the TIMS analyzer in each step (see Figure 7.1b). The mobility range that is injected into the FT-ICR MS analyzer is defined by the value of the voltage increment $\Delta V_{\text {ramp }}$ applied during a mobility step (Figure 7.2). The subsequent mobility range is defined by the step increment $\left(V_{\text {step }}\right)$ in the voltage across the ramp as $\Delta V_{\text {ramp }}^{\prime}=\Delta V_{\text {ramp }}+$ $V_{\text {step. }}$ During SA-TIMS operation the voltage range and the step increment are the same $\Delta V_{\text {ramp }}=V_{\text {step }}$. That is, the voltage range that is scanned by $V_{\text {ramp }}$ defines the mobility range, 
while the size of $\Delta V_{\text {ramp }}$ and $V_{\text {step }}$ determines how that range is sampled. Notice that in SATIMS the mobility resolution is limited to the voltage range in the ramp (and in the step). A typical $\Delta V_{\text {ramp }}$ of $0.5-2 \mathrm{~V}$ is utilized in SA-TIMS; ${ }^{63}$ the lower the range the higher the mobility resolution and the lower the sensitivity. In an alternative approach, if the $V_{\text {step }} \ll$ $\Delta V_{\text {ramp }}$, each mobility ion package can be sampled multiple times; this mode of operation is called oversampling SA-TIMS (OSA-TIMS). During OSA-TIMS, the number of measured points can be significantly increased across a mobility ion package which enables the use of more accurate molecular feature processing tools. In addition, the mobility resolution is no longer limited to the size of the $\Delta V_{\text {ramp }}$, is time-independent, and is mainly defined by the trapping conditions on the TIMS analyzer (e.g., bath gas velocity, electric field strength, and RF confinement).

\subsubsection{Mobility and Collision Cross Section Calculations}

The mobility of an ion can be calculated using first-principles in a TIMS analyzer. ${ }^{66-70}$ Each ion eluting after trapping from the TIMS cell can be described by a trapping window $E \pm \Delta E$ value that is directly related to the ion mobility $K \pm \Delta K$ and the velocity of the gas $v_{\mathrm{g}}$. Therefore, the mobility of an ion can be described by

$$
K=\frac{v_{g}}{E}=\frac{A}{V_{\text {elution }}-V_{\text {out }}}
$$

where $A$ is a calibration constant, $V_{\text {elution }}$ is the voltage in the $V_{\text {ramp }}$ sweep when ions elute, and $V_{\text {out }}$ is the voltage applied to the tunnel exit. The calibration constant $A$ was determined from previously reported mobility values for Tuning Mix calibration standard (G24221A, 
Agilent Technologies, Santa Clara, CA) in nitrogen $\left(m / z 322, K_{0}=1.376 \mathrm{~cm}^{2} \mathrm{~V}^{-1} \mathrm{~s}^{-1}\right.$ and $\left.m / z 622, K_{0}=1.013 \mathrm{~cm}^{2} \mathrm{~V}^{-1} \mathrm{~s}^{-1}\right) .68$

Reduced mobility values $\left(K_{0}\right)$ were correlated with $\operatorname{CCS}(\Omega)$ using the MasonSchamp equation:

$$
\Omega=\frac{(18 \pi)^{1 / 2}}{16} \frac{z e}{\left(k_{\mathrm{B}} T\right)^{1 / 2}}\left[\frac{1}{m_{\mathrm{l}}}+\frac{1}{m_{\mathrm{b}}}\right]^{1 / 2} \frac{1}{K_{0}} \frac{1}{N}
$$

where $z$ is the charge of the ion, $k_{\mathrm{B}}$ is the Boltzmann constant, $N$ is the number density, and $m_{\mathrm{I}}$ and $m_{\mathrm{b}}$ refer to the masses of the ion and bath gas, respectively. ${ }^{71}$

The mobility resolving power $(R)$ values reported herein were determined from Gaussian peak fits of the features in the TIMS distributions using OriginPro (version 8.0) by

$$
R=\operatorname{CCS} / \Delta \operatorname{CCS}(3)
$$

where CCS and $\triangle \mathrm{CCS}$ are the IMS peak center and fwhm, respectively.

\subsubsection{Experimental Parameters}

A custom-built, pulled capillary orthogonal nano electrospray ionization (nanoESI) source was utilized for the biomolecular experiments. Quartz glass capillaries (O.D.: 1.0 mm and I.D.: $0.70 \mathrm{~mm}$ ) were pulled utilizing a P-2000 micropipette laser puller (Sutter Instruments, Novato, CA) and loaded with a $10 \mu \mathrm{L}$ aliquot of the sample solution. A typical nanoESI source voltage of 600-1200 V was applied between the pulled capillary tips and the TIMS-FT-ICR MS instrument inlet. 
An orthogonal, commercial atmospheric pressure photoionization (APPI) source based on the Apollo II design (Bruker Daltonics, Inc., MA) was used. Briefly, sample solutions were introduced into the nebulizer at a rate of $300 \mu \mathrm{L} / \mathrm{min}$ using an external syringe pump. Typical APPI operating conditions were $900 \mathrm{~V}$ capillary voltage, $-900 \mathrm{~V}$ end-cap capillary offset voltage, $2 \mathrm{~L} / \mathrm{min}$ dry gas flow rate, 0.5 bar nebulizer gas pressure, a $220{ }^{\circ} \mathrm{C}$ dry gas temperature, and a $300{ }^{\circ} \mathrm{C}$ vaporizer temperature. Ions from the nanoESI and APPI source are introduced via a $0.6 \mathrm{~mm}$ inner diameter, single-bore glass capillary tube, which is resistively coated across its length, allowing the nebulizer to be maintained at ground potential, while the exit end of the capillary can be independently biased (typical values are $60-180 \mathrm{~V})$.

TIMS separation utilized nitrogen as a bath gas at about $300 \mathrm{~K}$ with the gas flow velocity controlled by the pressure difference between the entrance funnel $P_{1}=2.6-3.0$ mbar and the exit funnel $P_{2}=1.5-1.6$ mbar. TIMS voltage sequences were controlled using in-house software written in National Instruments Lab VIEW (2012, V. 12.0f3) and synchronized with the FT-ICR MS acquisition program. The TIMS cell was operated using a fill/trap/elute/quench sequence of 300/50/40/10 ms, collecting a single FT-ICR MS spectrum for each mobility trapping step. FT-ICR MS operation was optimized for ion transmission in the $m / z, 300-1500$ range and processed using sin-squared windowing in magnitude mode. For the evaluation of the SA-TIMS versus OSA-TIMS performance, a $\Delta V_{\text {ramp }}$ of $0.5,1$, and $5 \mathrm{~V}$ was used in the -25 to $-180 \mathrm{~V}$ range, a $V_{\text {step }}$ of $0.1,0.2,1$, and 5 $\mathrm{V}$, and single mobility experiments were accumulated in the collision cell prior to injection in the FT-ICR MS cell. The total analyst time for the SA-TIMS experiments was 73, 35, 
and $12 \mathrm{~s}$ for a $\Delta V_{\text {ramp }}$ of $0.5,1$, and $5 \mathrm{~V}$ compared to 5.2 min for a 400 step with $0.1 \mathrm{~V}$ acquisition OSA-TIMS experiments. For the analysis of the coal tar sample, multiple mobility steps $(\sim 30)$ were accumulated in the collision cell prior injection in the FT-ICR MS cell and acquired at 8-16Mword (6-12 s transient and $R_{\mathrm{MS}}=600-1200 \mathrm{k}$ at $\mathrm{m} / \mathrm{z} 400$, using an average of 100 scans $)$ and $512 \mathrm{kword}\left(R_{\mathrm{MS}}=35 \mathrm{k}\right.$ at $\mathrm{m} / \mathrm{z} 400$, using single scan $)$ for FT-ICR MS and TIMS-FT-ICR MS analysis, respectively.

\subsubsection{OSA-TIMS-FT-ICR MS Data Processing}

For targeted analysis, the reduced number of peaks of interest permits the manual extraction of the ion mobility spectra for accurate $m / z$ measurements $(<1 \mathrm{ppm})$ and further calibration of the OSA-TIMS scan step into mobility based on eq $\underline{1}$ using known mobility standards (see more details in refs 66-68). For untargeted analysis, the mobility calibration procedure is similar but the list of molecular ions of interest is generated from a single mass spectrum acquisition during a parallel acquisition using ultrahigh resolution FT-ICR MS with multiple averaging and longer transient times (e.g., 100 scan averages at 816Mword). The FT-ICR MS spectra are externally and internally calibrated using a Tuning Mix standard (Tunemix, G2421A, Agilent Technologies, Santa Clara, CA) and known polyaromatic hydrocarbon series, respectively. The peak lists are generated, allowing for a $\mathrm{S} / \mathrm{N}$ ratio of 6 . The formulas calculations from the exact mass domain are performed using Data Analysis Smart Formula package (Version v. 4.2, Bruker Daltonics, CA) with a maximum formula of $\mathrm{C}_{1-100} \mathrm{H}_{1-100} \mathrm{~N}_{0-2} \mathrm{O}_{0-2} \mathrm{~S}_{0-2}$, odd and even electron configurations allowed, and a mass tolerance of less than $1 \mathrm{ppm}$. The peak list is used for extraction of the ion mobility spectra from the OSA-TIMS-FT-ICR MS data set using batch processing in 
the data analysis package (Version v. 4.2, Bruker Daltonics, CA), followed by mobility calibration. Peak features are detected using a custom-built software package in Python v2.7 and Octave v4.0 from the mobility spectra using "asymmetric least squares smoothing" baseline correction, ${ }^{72}$ peak detection ("findpeak," and "peakfit.m" functions ${ }^{73}$, 74 using Gaussian fit functions with a fwhm criteria determined from mobility of known standards with less than $5 \%$ error as criteria for fit goodness), and a final generation of $[\mathrm{m} / \mathrm{z}$; chemical formula; $\mathrm{K} ; \mathrm{CCS}]$ data sets.

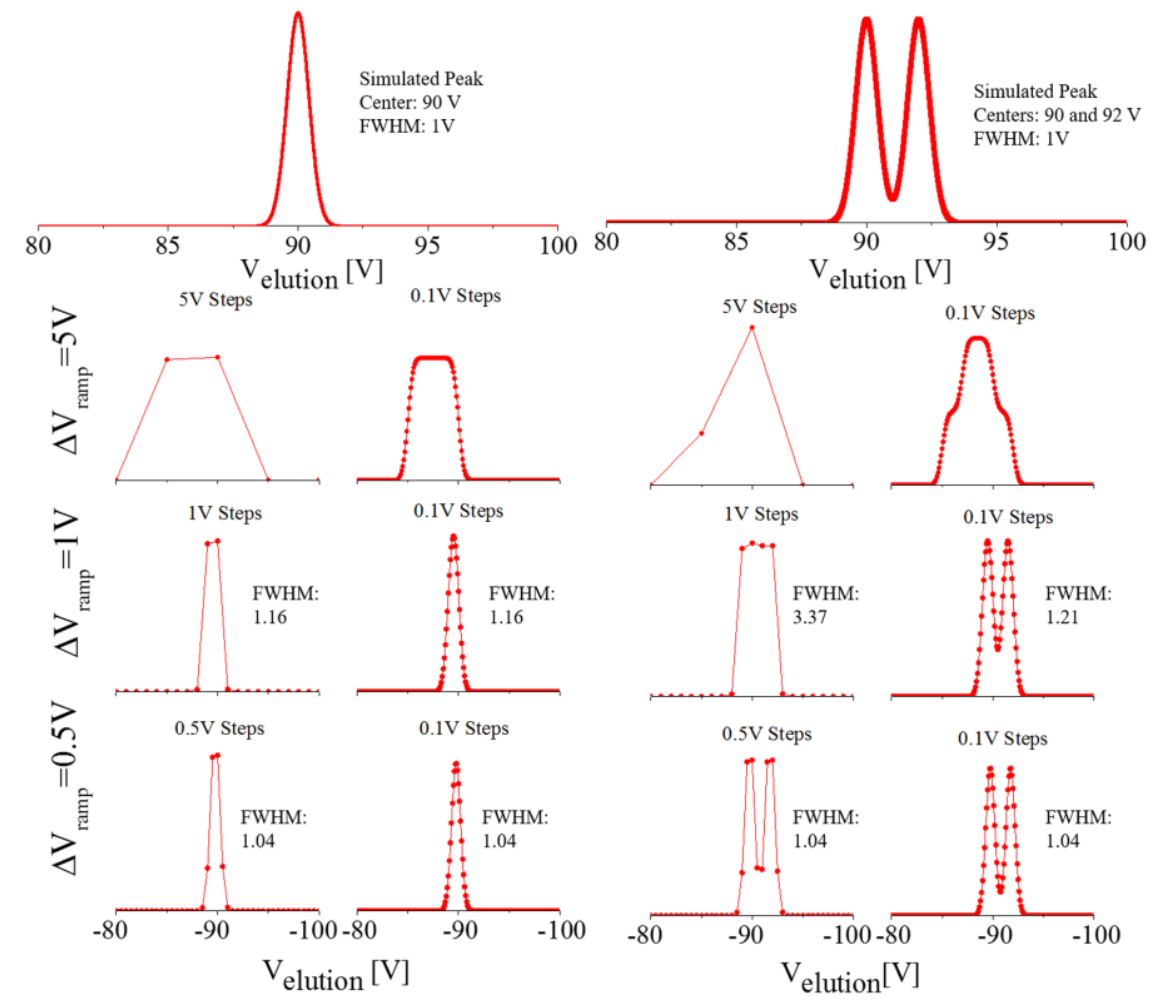

Figure 7.3. Theoretical IMS profiles utilizing a single and a double simulated peak with a fwhm of $1 \mathrm{~V}$ using selective accumulation SA (left) and oversampling OSA (right). Notice the number of points across the IMS peaks based on the trapping voltage $\left(\mathrm{V}_{\text {ramp }}\right)$ and the voltage steps $\left(\mathrm{V}_{\text {step }}\right)$ : SA-TIMS $\mathrm{V}_{\text {ramp }}=\mathrm{V}_{\text {step }}$ and OSATIMS $\mathrm{V}_{\text {ramp }} \gg \mathrm{V}_{\text {step. }}$.

\subsection{Results and Discussion}

The basis for OSA-TIMS relies on the fact that during TIMS analysis ion packages are spatially resolved based on their mobilities in the TIMS analyzer axis. Then, the question arises on how one can effectively sample an ion package in the TIMS analyzer 
axis using discrete elution steps. ${ }^{66-68,75}$ Since the mobility separation in the TIMS cell is time-independent, multiple strategies can be utilized during ion trapping and elution depending on the analytical challenge. An analogy can be made between OSA-TIMS and mass spectrometry imaging (MSI). For example, in MSI ion packages are injected into the MS analyzer from spatially resolved origins during independent events and it has been previously shown that oversampling (acquiring signal from adjacent positions spaced by distances smaller than the sample probe) is routinely used to avoid undersampling and to enhance the spatial imaging resolution. ${ }^{76,77}$ To further illustrate the OSA-TIMS process, simulations of the OSA-TIMS read-out from a theoretical peak centered at 90 and $1 \mathrm{~V}$ fwhm was studied as a function of the ramp and the step size voltages (Figure 7.3, left). Closer inspection of Figure 7.3 shows that $\Delta V_{\text {ramp }}$ influences the width of the read-out peak and the step size determines the number of points across the read-out peak. When $\Delta V_{\text {ramp }}$ $=V_{\text {step }}$ (SA-TIMS), the number of points across the read-out peak can be limited to few points if the width of the peak is on the order of the sampling step. For example, if $\Delta V_{\text {ramp }}$ $=5,1$, and $0.5 \mathrm{~V}$ the read-out peak has $1-2,2-4$, and $6-8$ points for a $1 \mathrm{~V}$ fwhm simulated peak during SA-TIMS, respectively; as the $\Delta V_{\text {ramp }}$ decreases the width of the read-out peak tends to more accurately reflect the original distribution. When $V_{\text {step }} \ll \Delta V_{\text {ramp }}$ (OSATIMS), the number of points across the peak increases, which permits better sampling of the original peak distribution. Theoretical simulations for the case of two peaks centered around 90 and $92 \mathrm{~V}(1 \mathrm{~V}$ fwhm) further illustrate the OSA-TIMS read out (Figure 7.3, right). This case, which is equivalent to two structural/conformational isomers, shows that the two signals can only be resolved when $\Delta V_{\text {ramp }}=0.5 \mathrm{~V}$ during SA-TIMS analysis or 
with $\Delta V_{\text {ramp }}=1-3 \mathrm{~V}$ with OSA-TIMS, while their separation further increases as $\Delta V_{\text {ramp }}$ decreases $\left(\Delta V_{\text {ramp }}=0.5 \mathrm{~V}\right)$.

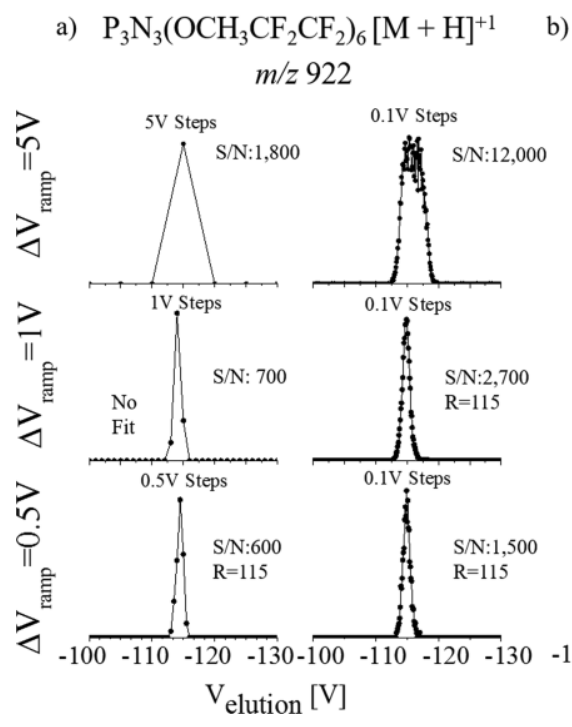

b)

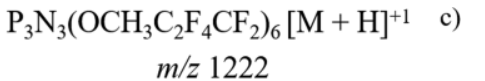

$\mathrm{P}_{3} \mathrm{~N}_{3}\left(\mathrm{OCH}_{3} \mathrm{C}_{3} \mathrm{~F}_{6} \mathrm{CF}_{2}\right)_{6}[\mathrm{M}+\mathrm{H}]^{+1}$
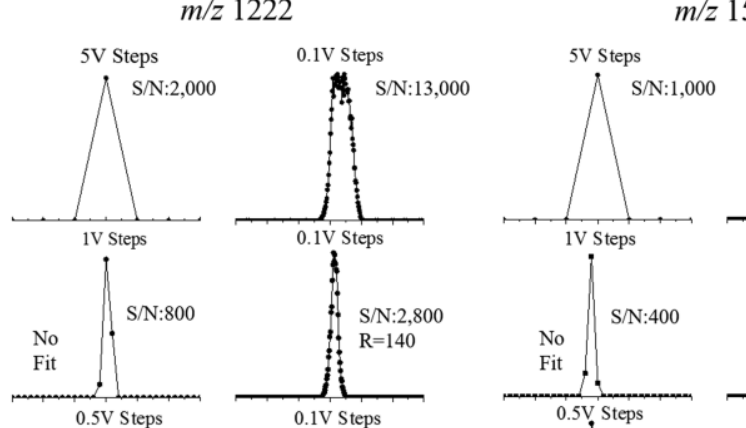

$z 1522$

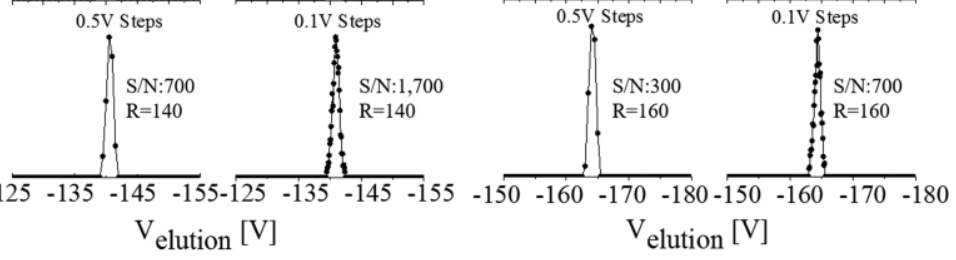

Figure 7.4. Typical experimental IMS profiles using selective accumulation SA (left) and oversampling OSA (right) TIMS-FT-ICR MS for the TuningMix standards at $\mathrm{m} / \mathrm{z}$ (a) 922, (b) 1222, and (c) 1522. Notice the differences in resolving power and signal-to-noise ratio between SA-TIMS and OSA-TIMS.

The OSA-TIMS concept was studied experimentally for the case of single and multiple mobility bands using known standards (Tuning Mix calibration standard $m / z$ 922, $m / z$ 1222, and $m / z$ 1522), structural peptide isomers (SDGRG and GRGDS reverse peptides) and conformational peptide isomers (ATHP-3). Good agreement was observed between the theoretical and the experimental single mobility bands (see Figures 7.3, left, and 7.4). Closer inspection of the Tuning Mix mobility spectra shows similar trends for the three $\mathrm{m} / z$ considered as a function of the $\Delta V_{\text {ramp }}$ and $\Delta V_{\text {step }}$ during SA-TIMS and OSATIMS (Figure 7.4). For example, the analyses showed a single band for all conditions considered; that is, no artifacts are introduced during OSA-TIMS. As the $\Delta V_{\text {ramp }}$ decreases a better agreement is observed between the peak shapes from SA-TIMS and OSA-TIMS mode of operations, without compromising the resolving power. However, the $\Delta V_{\text {ramp }}$ 
decrease is accompanied by a $\sim 3$-fold decrease in the signal-to-noise ratio from $\Delta V_{\text {ramp }}=$ 5 to $0.5 \mathrm{~V}$, while the signal-to-noise in the OSA-TIMS is typically 3-6x larger than in SATIMS. Notice that the number of points across the mobility band becomes analytically significant during OSA-TIMS (e.g., 15-21 points across a peak) with a $\Delta V_{\text {ramp }}=1-2 \mathrm{~V}$, while it may be challenging during SA-TIMS analysis (e.g., $\Delta V_{\text {ramp }}=\Delta V_{\text {step }}$ ).

The comparison of the theoretical and experimental profiles for multiple band mobilities also shows a good agreement (see Figures 7.3, right, and 7.5). The analysis of structural peptide isomers SDGRG and GRGDS, $[\mathrm{M}+\mathrm{H}]^{+}$, showed that they can be baseline
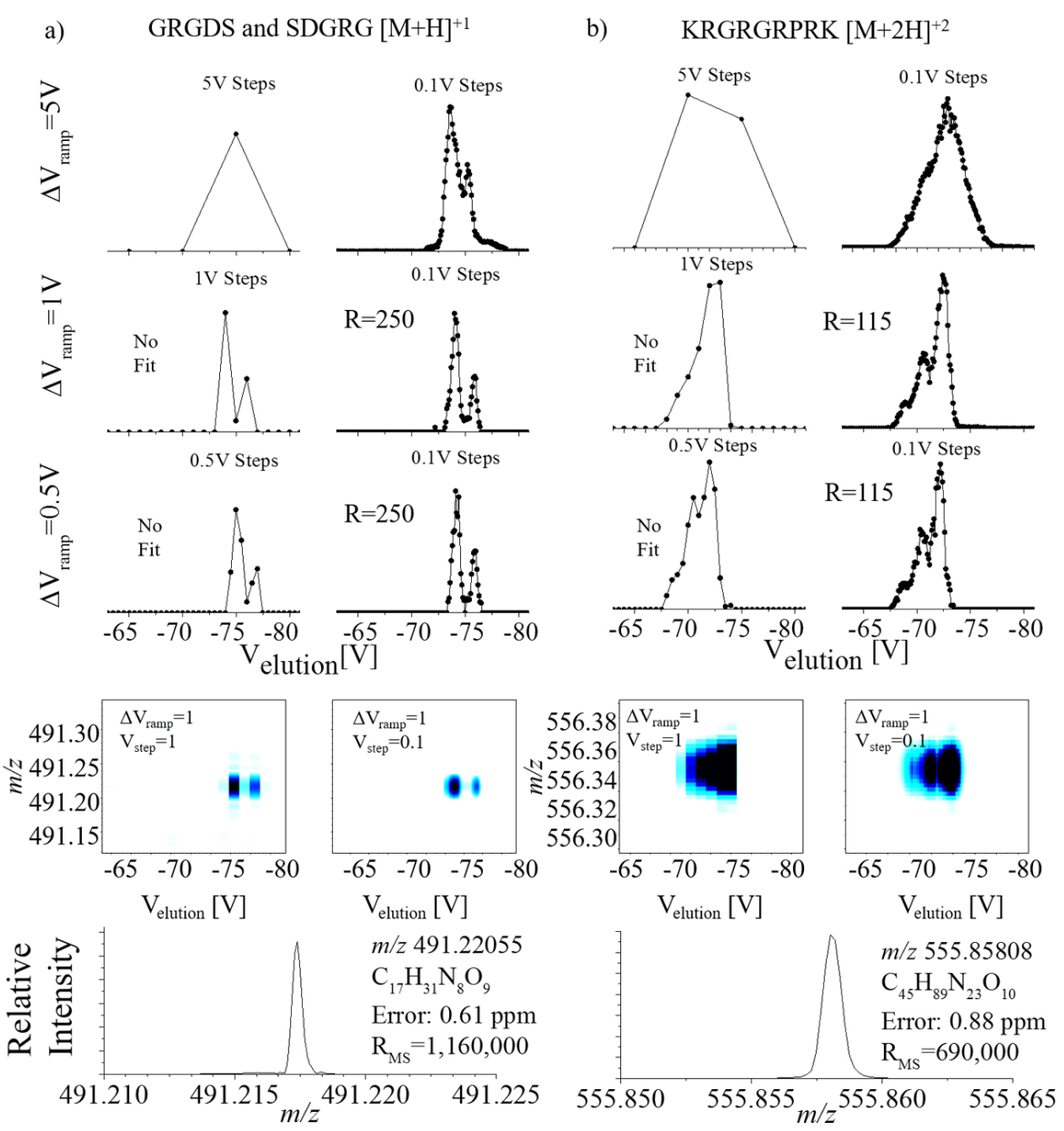

Figure 7.5. Typical IMS profiles using SA-TIMS (left) and OSA-TIMS (right) FT-ICR MS for (a) structural (SDGRG and GRGDS) and (b) conformational (ATHP 3) peptide isomers. Notice the high mass accuracy of the FT-ICR MS measurements. 
resolved using SA-TIMS and OSA-TIMS by increasing the velocity of the gas and optimization of the RF amplitude. During OSA-TIMS for the lower $\Delta V_{\text {ramp }}=0.5-1 \mathrm{~V}$, no artifacts were observed and a mobility resolving power of up to 250 (CCS/ACCS) was detected. Moreover, notice that there are no changes for the trapping values as a function of the $\Delta V_{\text {ramp }}(<1 \%)$, which permits accurate ion-neutral collision cross section calculations using first-principles (see eqs 1 and 2); collision cross sections of 200 and $203 \AA^{2}$ were detected for GRGDS and SDGRG $[\mathrm{M}+\mathrm{H}]^{+}$ions, in good agreement with previously reported values utilizing other IMS variants. ${ }^{78,79}$ The analysis of ATHP-3, $[\mathrm{M}+2 \mathrm{H}]^{+2}$, shows the potential of OSA-TIMS for the separation of conformational isomers without introducing artifacts (see Figure 7.5b). The detection and recognition of conformational peptide isomers during IMS analysis is challenging because there are dependencies on the solution starting conditions, molecular ion generation, and desolvation process, introduction into the IMS cell, IMS bath gas composition, and effective ion temperature during IMS separation. ${ }^{80-92}$ That is, in the case of conformational isomers the observation of multiple mobility bands has the prerequisite that (i) the conformational isomers were generated prior to IMS analysis and that they are stable during the IMS analysis (i.e., ions are thermalized to local minima in the free energy landscape during the IMS analysis) or (ii) conformational interconversion are energetically allowed during the IMS experiment. In any case, the mobility resolution plays a major role in the possibility to discern conformational isomers. For example, the structural flexibility of the biomolecule and the potential of conformational interconversion determines the mobility bandwidth; that is, the mobility bandwidth changes from case to case and a universal mobility bandwidth cannot be defined unless conformational interconversions are restricted during the IMS analysis. 
The major challenge during the study of conformational isomers is typically the assignment of mobility bands. Inspection of the ATHP-3, $[\mathrm{M}+2 \mathrm{H}]^{+2}$, during SA-TIMS analysis showed

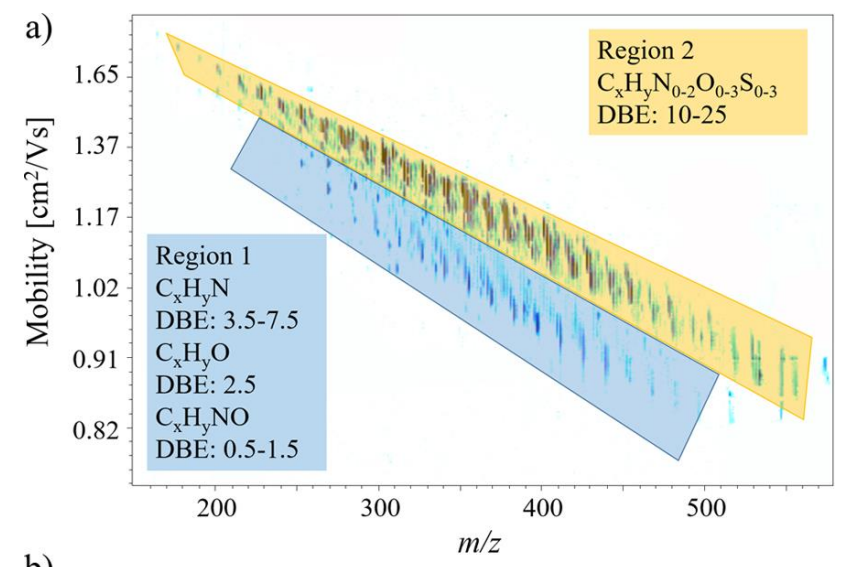

b)

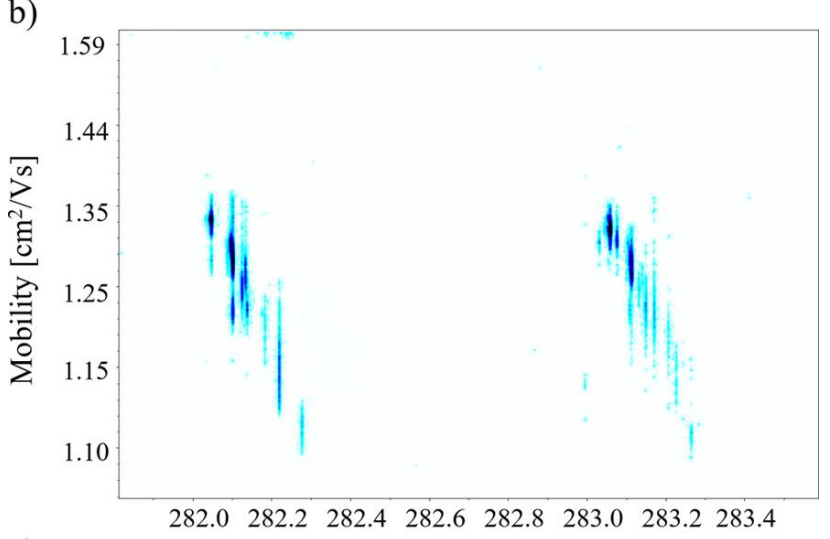

c)

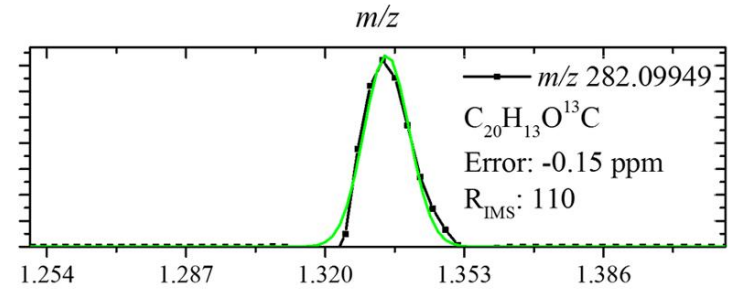

d)

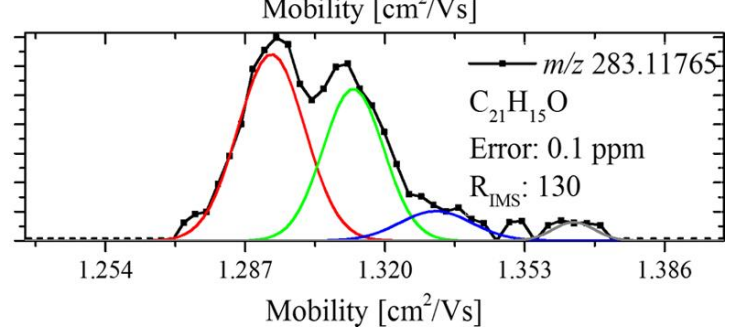

Figure 7.6. (a) Typical 2D-IMS-MS contour plot obtained using APPI-OSA-TIMS-FT-ICR MS from a complex mixture of coal tar. Two regions of interest are clearly discernible, resulting in a more aliphatic region (blue) and a more aromatic region (gold). (b) A closer view of $m / z$ 282-284 is shown, as well as the unsupervised fits for (c) a single and (d) multiple mobility band distributions. 
that the conformational space can be characterized by a broad mobility band distribution. Moreover, when the same molecular ion population was characterized using OSA-TIMS, the increase in the mobility resolution and number of points across the peak, by decreasing the $\Delta V_{\text {ramp }}=0.5-1 \mathrm{~V}$ with a small $\Delta V_{\text {step }}=0.1 \mathrm{~V}$, allowed the detection of three mobility bands with a resolving power of up to 120 . Notice that, in the process of reducing the $\Delta V_{\text {ramp }}$ values, changes in the trapping conditions did not occur, thus, allowing accurate CCS measurements without inducing changes in the distribution of the conformational isomers.

In addition to the previously described advantages of OSA-TIMS over traditional SA-TIMS when coupled to FT-ICR MS, one of the major analytical advantages of OSATIMS-FT-ICR MS architecture is the possibility to identify molecular features based on the mobility and $m / z$ domain. The ultrahigh mass resolution of the FT-ICR MS analyzers allows for the unsupervised generation of chemical formulas from complex mixtures based on the high $m / z$ separation and mass accuracy $(<1 \mathrm{ppm})$. When this is complemented with IMS separation and accurate CCS measurements, the OSA-TIMS-FT-ICR MS architecture allows for the unsupervised generation of candidate structures (see example in reference 65). This requires the separation of structural/conformational isomers using IMS, measuring the CCS, and assigning a chemical formula based on the MS measurement. The performance of OSA-TIMS-FT-ICR MS versus SA-TIMS-FT-ICR MS was evaluated for the analysis of a complex mixture of PAHs from coal tar (see Figure 7.6 and Table 7.1).

Dissection of the 2D-IMS-MS plots shows the increase in the number of molecular features detected from 2000 to 2800 and to 3800, comparing FT-ICR MS to SA-TIMS-FTICR MS and to OSA-TIMS-FT-ICR MS, respectively (see Appendix 7.1). Inspection of 
the 2D-IMS-MS of coal tar shows two main trend lines (regions 1 and 2 in Figure 7.6a). The separation of regions 1 and 2 in the IMS-MS space reduces the chemical noise and permits the generation of region specific MS projections of signals with lower abundance (e.g., see Appendix 7.2 where region 2 compounds are an order of magnitude less abundant than those detected in region 1). That is, this reduction in space charge effects and the increased peak capacity allows for greater sensitivity for the lower abundance species. Region 1 is composed primarily of a nitrogen containing compounds with a DBE 3.5-7.5, corresponding to alkyl pyridines and larger aromatics (e.g., phenyl-pyridine), as well as less-saturated molecules. Region 2 is composed primarily of aromatic and more condensed structures with a DBE range of 10-25. While SA-TIMS-FT-ICR MS represents an advantage over alternative strategies (e.g., parallel analysis by GCxGC-TOF, FT-ICR MS, and IMS-TOF-MS) for the separation of high mass isobars and isomers, the major drawback was in the possibility to separate closely related mobility bands due to the reduced number of points across the peak. With the introduction of OSA-TIMS-FT-ICR MS, the higher confidence of peak assignment during unsupervised processing of 2D-IMSMS data permits the generation of ( $\mathrm{m} / \mathrm{z}$; chemical formula; K; CCS) data sets from a single analysis. Unsupervised processing of the OSA-TIMS analysis of the coal tar mixture allows the detection of 3800 molecular features from 1800 assigned formulas. This is illustrated for the case of two mobility distributions between $m / z$ 282-284 (see Figure 7.6b-d). In this case, the increased number of points allows the automated peak fitting tools to accurately determine the profile for single and for multiple mobility band distributions (see example in Figure 7.6c,d). 
Table 7.1. List of Compounds Assigned Based on the Accurate Mass and Mobility from the 2D-IMS-MS Contour Plot Obtained Using APPI-OSA-TIMS-FT-ICR MS from a Complex Mixture of Coal Tar.

\begin{tabular}{|c|c|c|c|}
\hline $\mathrm{m} / \mathrm{z}$ & ion formula & error (ppm) & mobility $\left[\mathrm{cm}^{2} /(\mathrm{V} \mathrm{s})\right]$ \\
\hline 282.04981 & $\mathrm{C}_{20} \mathrm{H}_{10} \mathrm{~S}$ & -0.1 & 1.365 \\
\hline 282.09949 & $\mathrm{C}_{20} \mathrm{H}_{13} \mathrm{O}^{13} \mathrm{C}$ & -0.1 & 1.335 \\
\hline \multirow[t]{4}{*}{282.10394} & $\mathrm{C}_{21} \mathrm{H}_{14} \mathrm{O}$ & -0.09 & 1.121 \\
\hline & & & 1.250 \\
\hline & & & 1.301 \\
\hline & & & 1.314 \\
\hline \multirow[t]{3}{*}{282.12775} & $\mathrm{C}_{21} \mathrm{H}_{16} \mathrm{~N}$ & -0.1 & 1.142 \\
\hline & & & 1.269 \\
\hline & & & 1.286 \\
\hline \multirow[t]{3}{*}{282.13587} & $\mathrm{C}_{21} \mathrm{H}_{17}{ }^{13} \mathrm{C}$ & -0.1 & 1.224 \\
\hline & & & 1.285 \\
\hline & & & 1.306 \\
\hline 282.14035 & $\mathrm{C}_{22} \mathrm{H}_{18}$ & -0.18 & 1.253 \\
\hline \multirow[t]{4}{*}{282.22166} & $\mathrm{C}_{20} \mathrm{H}_{28} \mathrm{~N}$ & -0.1 & 1.090 \\
\hline & & & 1.160 \\
\hline & & & 1.183 \\
\hline & & & 1.206 \\
\hline \multirow[t]{3}{*}{282.27914} & $\mathrm{C}_{18} \mathrm{H}_{36} \mathrm{NO}$ & -0.01 & 1.120 \\
\hline & & & 1.132 \\
\hline & & & 1.228 \\
\hline 283.05763 & $\mathrm{C}_{20} \mathrm{H}_{11} \mathrm{~S}$ & -0.1 & 1.352 \\
\hline 283.07541 & $\mathrm{C}_{20} \mathrm{H}_{11} \mathrm{O}_{2}$ & -0.2 & 1.339 \\
\hline 283.10508 & $\mathrm{C}_{20} \mathrm{H}_{14} \mathrm{O}^{13} \mathrm{C}$ & 0.9 & 1.250 \\
\hline \multirow[t]{4}{*}{283.11176} & $\mathrm{C}_{21} \mathrm{H}_{15} \mathrm{O}$ & -0.1 & 1.293 \\
\hline & & & 1.314 \\
\hline & & & 1.332 \\
\hline & & & 1.364 \\
\hline 283.13105 & $\mathrm{C}_{20} \mathrm{H}_{16} \mathrm{~N}^{13} \mathrm{C}$ & 0.1 & 1.269 \\
\hline \multirow[t]{3}{*}{283.14817} & $\mathrm{C}_{22} \mathrm{H}_{19}$ & -0.2 & 1.151 \\
\hline & & & 1.245 \\
\hline & & & 1.264 \\
\hline \multirow[t]{4}{*}{283.16928} & $\mathrm{C}_{18} \mathrm{H}_{21} \mathrm{~N}_{2} \mathrm{O}$ & -0.7 & 1.164 \\
\hline & & & 1.238 \\
\hline & & & 1.259 \\
\hline & & & 1.339 \\
\hline 283.26317 & $\mathrm{C}_{18} \mathrm{H}_{35} \mathrm{O}_{2}$ & -0.03 & 1.123 \\
\hline
\end{tabular}

Notice that the specifics of OSA-TIMS-FT-ICR MS analysis (and corresponding analysis time) can be tailored by the sample complexity and relative abundance of the molecular features of interest. That is, the time-independent nature of the OSA-TIMS separation allows for the selective analysis of the mobility space of interest with varying degrees of mobility resolution and number of points across the peak; the higher the resolution and number of peaks, the longer the analysis time. In the case of the analysis of the coal tar by OSA-TIMS-FT-ICR MS, the analysis was completed in 85 min. In addition, 
as shown in the case of the PAH analysis, the number of OSA-TIMS steps that are accumulated and injected into the ICR cell can be tailored, which allows for better optimization of the ICR parameters, leading to higher mass resolution and better mass accuracy.

\subsection{Conclusions}

During TIMS analysis, ion packages are spatially resolved based on their mobilities along the TIMS analyzer axis and multiple strategies can be utilized to optimized the analytical applicability. In the present paper, we described the fundamentals and applicability of OSA-TIMS when coupled to ultrahigh resolution mass analyzers (e.g., FTICR MS). In particular, the analytical advantages during the analysis of structural/conformational isomers as well as for the unsupervised analysis of complex mixtures utilizing the mobility and $\mathrm{m} / \mathrm{z}$ domain were illustrated. Results showed that accurate ion-neutral collision cross sections $(<1 \%)$ can be measured using OSA-TIMS-FTICR MS with high mobility resolving powers $\left(R_{\mathrm{IMS}}\right.$ up to 250$)$, high mass accuracy $(<1$ ppm), and ultrahigh mass resolution ( $R_{\mathrm{MS}}$ up to $600-1200 \mathrm{k}$ at $\mathrm{m} / \mathrm{z} 400$ ) in a single analysis. A 2-fold increase in the number of molecular features and a 2-6-fold signal-to-noise increase were observed for OSA-TIMS when compared with SA-TIMS. This work provides the proof-of-principle for further application of OSA-TIMS-FT-ICR MS for the unsupervised analysis of complex mixtures based on the characterization of the conformational space and the assignment of chemical formulas in a single analysis.

\subsection{References}

1.Xian, F.; Hendrickson, C. L.; Marshall, A. G. Anal. Chem. 2012, 84, 708-719 
2.Nikolaev, E. N.; Kostyukevich, Y. I.; Vladimirov, G. N. Mass Spectrom. Rev. $2016,35,219-258$

3.Leach, F. E., III; Norheim, R.; Anderson, G.; Pasa-Tolic, L. J. Am. Soc. Mass Spectrom. 2014, 25, 2069-2072 126

4.Boldin, I. A.; Nikolaev, E. N. Rapid Commun. Mass Spectrom. 2011, 25, $122-$

5.Frankevich, V.; Zenobi, R. Int. J. Mass Spectrom. 2001, 207, 57- 67

6.Weisbrod, C. R.; Kaiser, N. K.; Skulason, G. E.; Bruce, J. E. Anal. Chem. 2010, $82,6281-6286$

7.Weisbrod, C. R.; Kaiser, N. K.; Skulason, G. E.; Bruce, J. E. Anal. Chem. 2008, $80,6545-6553$

8.Brustkern, A. M.; Rempel, D. L.; Gross, M. L. J. Am. Soc. Mass Spectrom. 2008, $19,1281-1285$

9.Brustkern, A. M.; Rempel, D. L.; Gross, M. L. J. Am. Soc. Mass Spectrom. 2010, $21,451-454$

10. Nikolaev, E. N.; Boldin, I. A.; Jertz, R.; Baykut, G. J. Am. Soc. Mass Spectrom. 2011, 22, 1125- 1133

11. Kostyukevich, Y. I.; Vladimirov, G. N.; Nikolaev, E. N. J. Am. Soc. Mass Spectrom. 2012, 23, 2198- 2207

12. Kaiser, N. K.; Savory, J. J.; McKenna, A. M.; Quinn, J. P.; Hendrickson, C. L.; Marshall, A. G. Anal. Chem. 2011, 83, 6907-6910

13. Tolmachev, A. V.; Robinson, E. W.; Wu, S.; Smith, R. D.; Pasa-Toli, L. J. Am. Soc. Mass Spectrom. 2011, 22, 1334- 1342

14. Tolmachev, A. V.; Robinson, E. W.; Wu, S.; Pasa-Tolic, L.; Smith, R. D. Int. J. Mass Spectrom. 2009, 287, 32- 38

15. Tolmachev, A. V.; Robinson, E. W.; Smith, R. D.; Leach, F. E.; Futrell, J. H.; Paša-Tolić, L. Int. J. Mass Spectrom. 2012, 325, 45- 50

16. Nagornov, K. O.; Kozhinov, A. N.; Tsybin, O. Y.; Tsybin, Y. O. J. Am. Soc. Mass Spectrom. 2015, 26, 741-751

17. Tolmachev, A. V.; Robinson, E. W.; Wu, S.; Kang, H.; Lourette, N. M.; PasaTolic, L.; Smith, R. D. J. Am. Soc. Mass Spectrom. 2008, 19, 586- 597 
18. Tolmachev, A. V.; Robinson, E. W.; Wu, S.; Smith, R. D.; Futrell, J. H.; PasaTolic, L. J. Am. Soc. Mass Spectrom. 2012, 23, 1169- 1172

19. Popov, I. A.; Nagornov, K.; Vladimirov, G. N.; Kostyukevich, Y. I.; Nikolaev, E. N. J. Am. Soc. Mass Spectrom. 2014, 25, 790- 799

20. Marshall, A. G.; Guan, S. Rapid Commun. Mass Spectrom. 1996, 10, 18191823

21. Hendrickson, C. L.; Quinn, J. P.; Kaiser, N. K.; Smith, D. F.; Blakney, G. T.; Chen, T.; Marshall, A. G.; Weisbrod, C. R.; Beu, S. C. J. Am. Soc. Mass Spectrom. 2015, 26, 1626- 1632

22. Cho, Y.; Qi, Y.; O’Connor, P. B.; Barrow, M. P.; Kim, S. J. Am. Soc. Mass Spectrom. 2014, 25, 154- 157

23. Qi, Y.; Li, H.; Wills, R. H.; Perez-Hurtado, P.; Yu, X.; Kilgour, D. P.; Barrow, M. P.; Lin, C.; O’Connor, P. B. J. Am. Soc. Mass Spectrom. 2013, 24, 828- 834

24. Qi, Y.; Witt, M.; Jertz, R.; Baykut, G.; Barrow, M. P.; Nikolaev, E. N.; O’Connor, P. B. Rapid Commun. Mass Spectrom. 2012, 26, 2021- 2026

25. Qi, Y.; Barrow, M. P.; Li, H.; Meier, J. E.; Van Orden, S. L.; Thompson, C. J.; O’Connor, P. B. Anal. Chem. 2012, 84, 2923- 2929

26. Kilgour, D. P.; Nagornov, K. O.; Kozhinov, A. N.; Zhurov, K. O.; Tsybin, Y. O. Rapid Commun. Mass Spectrom. 2015, 29, 1087- 1093

27. Kilgour, D. P.; Wills, R.; Qi, Y.; O’Connor, P. B. Anal. Chem. 2013, 85, 39033911

28. Nakata, M. T.; Hart, G. W.; Peterson, B. G. J. Am. Soc. Mass Spectrom. 2010, $21,1712-1719$

29. Mitchell, D. W.; Smith, R. D. Phys. Rev. E: Stat. Phys., Plasmas, Fluids, Relat. Interdiscip. Top. 1995, 52, 4366- 4386

30. Boldin, I. A.; Nikolaev, E. N. Rapid Commun. Mass Spectrom. 2009, 23, 32133219

31. Nikolaev, E. N. Int. J. Mass Spectrom. 2015, 377, 421- 431

32. Comisarow, M. B.; Marshall, A. G. J. Chem. Phys. 1976, 64, 110- 119

33. Guan, S.; Li, G.-Z.; Marshall, A. G. Int. J. Mass Spectrom. Ion Processes 1997, $167,185-193$ 
34. Mitchell, D. W. J. Am. Soc. Mass Spectrom. 1999, 10, 136- 152

35. Nikolaev, E. N.; Heeren, R. M.; Popov, A. M.; Pozdneev, A. V.; Chingin, K. S. Rapid Commun. Mass Spectrom. 2007, 21, 3527-3546

36. Stafford, G. C., Jr; Taylor, D. M.; Bradshaw, S. C. US5107109 A, 1992.

37. Senko, M. W.; Hendrickson, C. L.; Emmett, M. R.; Shi, S. D.-H.; Marshall, A. G. J. Am. Soc. Mass Spectrom. 1997, 8, 970- 976

38. Belov, M. E.; Anderson, G. A.; Angell, N. H.; Shen, Y.; Tolic, N.; Udseth, H. R.; Smith, R. D. Anal. Chem. 2001, 73, 5052- 5060

39. Paša-Tolić, L.; Harkewicz, R.; Anderson, G. A.; Tolić, N.; Shen, Y.; Zhao, R.; Thrall, B.; Masselon, C.; Smith, R. D. J. Am. Soc. Mass Spectrom. 2002, 13, 954- 963

40. Harkewicz, R.; Belov, M. E.; Anderson, G. A.; Pasa-Tolic, L.; Masselon, C. D.; Prior, D. C.; Udseth, H. R.; Smith, R. D. J. Am. Soc. Mass Spectrom. 2002, 13, 144- 154

41. Bruce, J. E.; Anderson, G. A.; Hofstadler, S. A.; Van Orden, S. L.; Sherman, M. S.; Rockwood, A. L.; Smith, R. D. Rapid Commun. Mass Spectrom. 1993, 7, 914-919

42. van Agthoven, M. A.; Barrow, M. P.; Chiron, L.; Coutouly, M. A.; Kilgour, D.; Wootton, C. A.; Wei, J.; Soulby, A.; Delsuc, M. A.; Rolando, C.; O’Connor, P. B. J. Am. Soc. Mass Spectrom. 2015, 26, 2105-2114

43. van Agthoven, M. A.; Chiron, L.; Coutouly, M. A.; Delsuc, M. A.; Rolando, C. Anal. Chem. 2012, 84, 5589-5595

44. van Agthoven, M. A.; Chiron, L.; Coutouly, M.-A.; Sehgal, A. A.; Pelupessy, P.; Delsuc, M.-A.; Rolando, C. Int. J. Mass Spectrom. 2014, 370, 114- 124

45. van Agthoven, M. A.; Delsuc, M. A.; Bodenhausen, G.; Rolando, C. Anal. Bioanal. Chem. 2013, 405, 51- 61

46. van Agthoven, M. A.; Delsuc, M.-A.; Rolando, C. Int. J. Mass Spectrom. 2011, 306, 196- 203

47. van Agthoven, M. A.; Wootton, C. A.; Chiron, L.; Coutouly, M. A.; Soulby, A.; Wei, J.; Barrow, M. P.; Delsuc, M. A.; Rolando, C.; O'Connor, P. B. Anal. Chem. 2016, 88, 4409- 4417

48. Dunbar, R. C. Mass Spectrom. Rev. 2004, 23, 127- 158

49. Laskin, J. Principles of Mass Spectrometry Applied to Biomolecules; John Wiley \& Sons, Inc.: Hoboken, NJ, U.S.A., 2006; pp 619- 665. 
50. Klassen, J. S.; Schnier, P. D.; Williams, E. R. J. Am. Soc. Mass Spectrom. 1998, $9,1117-1124$

51. Felitsyn, N.; Kitova, E. N.; Klassen, J. S. Anal. Chem. 2001, 73, 4647-4661

52. Barrow, M. P.; Peru, K. M.; Headley, J. V. Anal. Chem. 2014, 86, 8281-8288

53. Lababidi, S.; Panda, S. K.; Andersson, J. T.; Schrader, W. Anal. Chem. 2013, $85,9478-9485$

54. Lababidi, S.; Schrader, W. Rapid Commun. Mass Spectrom. 2014, 28, 13451352

55. Benigni, P.; DeBord, J. D.; Thompson, C. J.; Gardinali, P.; Fernandez-Lima, F. Energy Fuels 2016, 30, 196-203

56. Kailemia, M. J.; Park, M.; Kaplan, D. A.; Venot, A.; Boons, G. J.; Li, L.; Linhardt, R. J.; Amster, I. J. J. Am. Soc. Mass Spectrom. 2014, 25, 258- 268

57. Robinson, E. W.; Leib, R. D.; Williams, E. R. J. Am. Soc. Mass Spectrom. 2006, 17, 1469- 1479

58. Robinson, E. W.; Sellon, R. E.; Williams, E. R. Int. J. Mass Spectrom. 2007, $259,87-95$

59. Robinson, E. W.; Williams, E. R. J. Am. Soc. Mass Spectrom. 2005, 16, 1427143

60. Robinson, E. W.; Garcia, D. E.; Leib, R. D.; Williams, E. R. Anal. Chem. 2006, $78,2190-2198$

61. Tang, X.; Bruce, J. E.; Hill, H. H., Jr. Rapid Commun. Mass Spectrom. 2007, $21,1115-1122$

62. Bluhm, B. K.; Gillig, K. J.; Russell, D. H. Rev. Sci. Instrum. 2000, 71, 40784086

63. Benigni, P.; Thompson, C. J.; Ridgeway, M. E.; Park, M. A.; Fernandez-Lima, F. Anal. Chem. 2015, 87, 4321-4325

64. Pu, Y.; Ridgeway, M. E.; Glaskin, R. S.; Park, M. A.; Costello, C. E.; Lin, C. Anal. Chem. 2016, 88, 3440-3443

65. Benigni, P.; Marin, R.; Fernandez-Lima, F. Int. J. Ion Mobility Spectrom. 2015, $18,151-157$ 
66. Fernandez-Lima, F. A.; Kaplan, D. A.; Park, M. A. Rev. Sci. Instrum. 2011, 82, 126106

67. Fernandez-Lima, F.; Kaplan, D. A.; Suetering, J.; Park, M. A. Int. J. Ion Mobility Spectrom. 2011, 14, 93-98

68. Hernandez, D. R.; Debord, J. D.; Ridgeway, M. E.; Kaplan, D. A.; Park, M. A.; Fernandez-Lima, F. Analyst 2014, 139, 1913- 1921

69. Benigni, P.; Marin, R.; Molano-Arevalo, J. C.; Garabedian, A.; Wolff, J. J.; Ridgeway, M. E.; Park, M. A.; Fernandez-Lima, F. Int. J. Ion Mobility Spectrom. 2016, 110

70. Adams, K. J.; Montero, D.; Aga, D.; Fernandez-Lima, F. Int. J. Ion Mobility Spectrom. 2016, 1-8

71. McDaniel, E. W.; Mason, E. A. Mobility and Diffusion of Ions in Gases; John Wiley and Sons, Inc., New York, 1973; p 381.

72. Eilers, P. H.; Boelens, H. F. Leiden University Medical Centre Report, 2005.

73. O'Haver, T. A Pragmatic Introduction to Signal Processing, 2nd ed.; Self published, Printed by CreateSpace Independent Publishing Platform, 2016.

74. Thomas C, O. H. Interactive Peak Fitter, https://terpconnect.umd.edu/ toh/spectrum/InteractivePeakFitter.htm (accessed Apr, 11, 2016) .

75. Ridgeway, M. E.; Wolff, J. J.; Silveira, J. A.; Lin, C.; Costello, C. E.; Park, M. A. Int. J. Ion Mobility Spectrom. 2016, 1-9

76. Todd, P. J.; Schaaff, T. G.; Chaurand, P.; Caprioli, R. M. J. Mass Spectrom. $2001,36,355-369$

77. Jurchen, J. C.; Rubakhin, S. S.; Sweedler, J. V. J. Am. Soc. Mass Spectrom. $2005,16,1654-1659$

78. Giles, K.; Williams, J. P.; Campuzano, I. Rapid Commun. Mass Spectrom. 2011, 25, 1559- 1566

79. Bush, M. F.; Hall, Z.; Giles, K.; Hoyes, J.; Robinson, C. V.; Ruotolo, B. T. Anal. Chem. 2010, 82, 9557-9565

80. Schenk, E. R.; Almeida, R.; Miksovska, J.; Ridgeway, M. E.; Park, M. A.; Fernandez-Lima, F. J. Am. Soc. Mass Spectrom. 2015, 26, 555- 563 
81. McKenzie-Coe, A.; DeBord, J. D.; Ridgeway, M.; Park, M.; Eiceman, G.; Fernandez-Lima, F. Analyst 2015, 140, 5692- 5699

82. Schenk, E. R.; Ridgeway, M. E.; Park, M. A.; Leng, F.; Fernandez-Lima, F. Anal. Chem. 2014, 86, 1210- 1214

83. Molano-Arevalo, J. C.; Hernandez, D. R.; Gonzalez, W. G.; Miksovska, J.; Ridgeway, M. E.; Park, M. A.; Fernandez-Lima, F. Anal. Chem. 2014, 86, 10223- 10230

84. Fernandez-Lima, F. A.; Wei, H.; Gao, Y. Q.; Russell, D. H. J. Phys. Chem. A $2009,113,8221-8234$

85. Morsa, D.; Gabelica, V.; De Pauw, E. J. Am. Soc. Mass Spectrom. 2014, 25, $1384-1393$

86. Morsa, D.; Gabelica, V.; De Pauw, E. Anal. Chem. 2011, 83, 5775- 5782

87. Shi, L.; Holliday, A. E.; Glover, M. S.; Ewing, M. A.; Russell, D. H.; Clemmer, D. E. J. Am. Soc. Mass Spectrom. 2016, 27, 22- 30

88. Shi, H.; Clemmer, D. E. J. Phys. Chem. B 2014, 118, 3498- 3506

89. Silveira, J. A.; Fort, K. L.; Kim, D.; Servage, K. A.; Pierson, N. A.; Clemmer, D. E.; Russell, D. H. J. Am. Chem. Soc. 2013, 135, 19147- 19153

90. Shi, H.; Pierson, N. A.; Valentine, S. J.; Clemmer, D. E. J. Phys. Chem. B 2012, $116,3344-3352$

91. Pierson, N. A.; Chen, L.; Valentine, S. J.; Russell, D. H.; Clemmer, D. E. J. Am. Chem. Soc. 2011, 133, 13810- 13813

92. Pierson, N. A.; Valentine, S. J.; Clemmer, D. E. J. Phys. Chem. B 2010, 114, $7777-7783$ 
CHAPTER VIII

\section{ANALYSIS OF PHOTOIRRADIATED WATER ACCOMMODATED FRACTIONS OF CRUDE OILS USING TANDEM TIMS AND FT-ICR MS}

(Adapted with permission from Benigni et al., 2017, Environmental Science and Technology, copyright 2017 American Chemical Society) 


\subsection{Abstract}

For the first time, trapped ion mobility spectrometry (TIMS) in tandem with Fourier transform ion cyclotron resonance mass spectrometry (FT-ICR MS) is applied to the analysis of the low energy water accommodated fraction (WAF) of a crude oil as a function of the exposure to light. The TIMS-FT-ICR MS analysis provided, in addition to the heteroatom series identification, new insights into the WAF isomeric complexity (e.g., [m/z; chemical formula; collision cross section] data sets) for a better evaluation of the degree of chemical and structural photoinduced transformations. Inspection of the $[\mathrm{m} / \mathrm{z}$; chemical formula; collision cross section] data sets shows that the WAF composition changes as a function of the exposure to light in the first $115 \mathrm{~h}$ by initial photosolubilization of $\mathrm{HC}$ components and their photo-oxidation up to $\mathrm{O}_{4-5}$ of mainly high double bond equivalence species (DBE > 9). The addition of high resolution TIMS (resolving power of 90-220) to ultrahigh resolution FT-ICR MS (resolving power over 400k) permitted the identification of a larger number of molecular components in a single analysis (e.g., over 47k using TIMS-MS compared to $12 \mathrm{k}$ by MS alone), with instances of over 6-fold increase in the number of molecular features per nominal mass due to the WAF isomeric complexity. This work represents a stepping stone toward a better understanding of the WAF components and highlights the need for better experimental and theoretical approaches to characterize the WAF structural diversity.

\subsection{Introduction}

The complex nature of crude oil and its incorporation in aquatic systems results in

complex chemical transformations mainly via bio- ${ }^{1-8}$ and photo degradation. ${ }^{9-14}$ Natural 
and anthropogenic release of crude oil and hydrocarbons ${ }^{15,16}$ to seawater is a frequent process, and recent studies have highlighted the importance of characterizing released crude oil at the molecular level. ${ }^{17}$ For example, the characterization of heteroatom $(\mathrm{O}, \mathrm{N}$, and S) polyaromatic hydrocarbons (h-PAHs) has exhibited increased levels of toxicity compared to pure hydrocarbons. ${ }^{18}$ Moreover, PAHs are photo active, undergoing oxygenation upon exposure to light, which can lead to chemical products that have increased biological accumulation and activity. ${ }^{19-25}$ The presence of crude oil in water provides the means for the exposure of a large number of molecules to chemical and enzymatic transformation and their transportation across environments, ${ }^{26-28}$ as well as the interaction with a variety of organisms. ${ }^{26}$ Many of these chemical changes, as well as the means of exposure to organisms, occur in the water accommodated fraction, where low energy mixing introduces components of the oil into the water, without the formation of detectable emulsions. ${ }^{29}$ The main analytical challenge during the analysis of the low energy water accommodated fraction (WAF) remains the identification and quantitation of both the primary molecular species, as well as the transformation intermediates and products.

Over the past years, most of the efforts for the WAF analysis have been focused on the use of gas chromatography-mass spectrometry (GC-MS) $7,9,10,13,30-36$ and twodimensional gas chromatography (GCXGC-MS) with heavy standards. ${ }^{31,37}$ While progress has been made in the WAF characterization, these approaches are limited to the volatility range of molecules that can be analyzed by GC, which typically excludes large and highly polar molecules. ${ }^{38}$ These analytical limitations narrow the type of studies that can be performed and our understanding of the crude oil transformations in seawater since the 
molecular species that are inaccessible or form unresolvable "humps", known as the unresolved complex mixture (UCM), can make up most of the WAF content. ${ }^{39,}{ }^{40}$ The analytical challenges associated with the molecular characterization of the UCM has led to the use of alternative tools in order to unravel its chemical complexity. For example, studies utilizing ultrahigh mass resolution mass spectrometry (e.g., FT-ICR MS) ${ }^{41-44}$ have enabled the identification of chemical formulas using the isotopic resolution and the high mass accuracy (sub ppm) with a variety of atmospheric pressure ionization sources (e.g., electrospray ionization, ESI, ${ }^{45,46}$ atmospheric pressure chemical ionization, APCI, ${ }^{47-49}$ atmospheric pressure photoionization, APPI, ${ }^{50-53}$ and atmospheric pressure laser ionization, $\left.\mathrm{APLI}^{54-61}\right)$. The use of a variety of atmospheric pressure ionization sources has enabled, in turn, the study of different molecular fractions at the molecular level and has provided evidence of the high structural diversity of the WAF components in their functional groups, aromaticity, and polarity. ${ }^{62-64}$

The high structural diversity of the WAF samples has prompted the need to complement ultrahigh resolution mass analysis (e.g., FT-ICR MS measurements) with preseparation techniques (e.g., liquid and gas chromatography), in order to better discriminate the components along a second axis of separation, permitting some isomeric separation and increasing the dynamic range of the FT-ICR MS measurement. ${ }^{59,60,65-69}$ However, the biggest challenge in the coupling of liquid and gas chromatography is that it limits the FT-ICR MS analysis time, and thus the ability to better separate isobaric species, due to the slow acquisition rates needed for ultrahigh resolution mass acquisitions. ${ }^{48,65}$ Alternatively, other post-ionization, gas-phase separations have been proven to be a better 
match for FT-ICR MS analysis. ${ }^{70-79}$ In particular, ion mobility spectrometry (IMS) presents many advantages for the analysis of complex mixtures, providing orthogonal separation to FT-ICR MS that is based on the tridimensional structure of the molecule. ${ }^{80-82}$ Initial work showed the potential of IMS-MS analysis for the characterization of complex hydrocarbon mixtures using complementary IMS-MS and FT-ICR MS measurements (e.g., IMS-TOF MS and FT-ICR MS of the same sample). ${ }^{83-92}$ More recently, with the development of trapped ion mobility spectrometry (TIMS), ${ }^{93-95}$ several reports have shown the potential of TIMS-MS to decouple the mobility $(\mathrm{K})$ separation from the MS analysis time for fast, gasphase separation and for molecular structural elucidation. ${ }^{51,93,96-117}$ In particular, the advantages of TIMS over traditional IMS analyzers have been shown for fast screening ${ }^{96}$ and targeted ${ }^{79}$ analysis of molecular ions from complex chemical mixtures; the study of isomerization kinetics of small molecules ${ }^{98}$ peptides, DNA, proteins, and their complexes in the absence of the bulk solvent; $;^{99-103}$ the influence of the collision partner on the molecular structure, ${ }^{104}$ and the factors that affect molecular-adduct complex lifetime and stability during TIMS measurements. ${ }^{105}$ A significant feature to note is the high resolving power of TIMS analyzers $\left(R_{\text {TIMS }}\right.$ up to $\left.400^{107}\right)$ and accuracy in measuring ion-neutral collision cross section (CCS, $<0.6 \%$ error). In the case of crude oils and complex mixtures, their characterization by TIMS-FT-ICR MS has allowed the measurement of the CCS, accurate mass, and accurate isotopic fine structure in a single experiment for a series of $\mathrm{h}$ PAHs. For example, a recent report of Oversampling Selective Accumulation Trapped Ion Mobility Spectrometry (OSA-TIMS) coupled to FT-ICR MS showed high mobility resolving powers (over 250), high mass accuracy $(<1 \mathrm{ppm})$, and ultrahigh mass resolution 
(up to $1,200,000$ at $\mathrm{m} / \mathrm{z} 400$ ) during the analysis of a complex mixture of polyaromatic hydrocarbons (PAH) from coal tar. ${ }^{106}$

In the present work, for the first time, we apply tandem OSA-TIMS and FT-ICR MS for the analysis of WAF samples as a function to their exposure to light. While preliminary work has shown that WAF can undergo chemical transformations and increase oil solubilization as a function to the exposure to light, ${ }^{43}$ little is known about the WAF structural variability and transformation pathways. In this study, as an initial step, the analysis focuses on the PAH compounds accessible to ionization by an APLI source (e.g., mostly conjugated molecules) which typically exhibits higher reactivity to light resulting in more hazardous byproducts. ${ }^{118}$ In addition to the new analytical advantages of TIMSFT-ICR MS, a Software Assisted Molecular Elucidation (SAME) package was developed for the unsupervised processing of the OSA-TIMS-FT-ICR MS data sets. As shown below, this work highlights the need for high mobility resolution and ultrahigh resolution MS for the analysis of the highly isomeric, complex WAF mixtures while providing $[\mathrm{m} / \mathrm{z}$; chemical formula; $\mathrm{K} ; \mathrm{CCS}]$ in a single experiment.

\subsection{Experimental Section}

\subsubsection{Sample Preparation}

Low-energy water accommodated fractions (WAF) were generated according to the standardized protocol established by the Chemical Response to Oil Spills: Ecological

Research Forum (CROSERF). ${ }^{119,}{ }^{120}$ Briefly, WAFs were prepared at room temperature in 2-L aspirator bottles with $20 \%$ headspace by volume using artificial filtered saltwater (pore size: $0.45 \mu \mathrm{m}$, salinity $=33$ parts-per-thousand) prepared with Instant Ocean (Aquarium 
Systems, Mentor OH). Oil from the Marlin Platform was added to the water surface using a gastight syringe at an oil-to-water loading of 1:1000 (1 g oil/L seawater). The bottles were covered in aluminum foil and allowed to mix for $24 \mathrm{~h}$ at low speed (100 rpm) in the dark.

\subsubsection{WAF exposure to Light and Extraction}

WAF samples were irradiated up to $115 \mathrm{~h}$ with a Suntest XLS+ Sunlight Exposure System equipped with a $1500 \mathrm{~W}$ xenon arc lamp and light intensity of $765 \mathrm{~W} / \mathrm{m}^{2}$ (Atlas,

Chicago, IL, USA). The aspirator bottles containing the WAF and the oil were placed in a water bath system set to $25^{\circ} \mathrm{C}$. At specific irradiation times $(0,16$, and $115 \mathrm{~h}), 150 \mathrm{~mL}$ aliquots of the WAF were removed and subjected to liquid-liquid extraction in three 50 $\mathrm{mL}$ lots of methylene chloride to increase extraction efficiency. Aliquots were dried over $\mathrm{Na}_{2} \mathrm{SO}_{4}$ and concentrated down to $1 \mathrm{~mL}$ under a stream of nitrogen. The final samples were then diluted 1:100 in 1:1 v/v methanol/toluene for FT-ICR MS and TIMS-FT-ICR MS analysis.

\subsubsection{FT-ICR MS analysis}

WAF samples were analyzed in positive ion mode with an APLI source coupled to a custom-built TIMS - FT-ICR MS instrument based on the 7T Solarix FT-ICR MS spectrometer (Bruker Daltonics Inc., MA). Briefly, the APLI source utilizes a $266 \mathrm{~nm}$ excimer laser (CryLas GmbH, Berlin, Germany; Type:1HP266-50); the sample was introduced at $200 \mu \mathrm{L} / \mathrm{h}$ through a short nebulizer in a vaporizer set to $300^{\circ} \mathrm{C}$ into the source chamber where the gas stream was ionized by the excimer laser. ${ }^{65}$ The APLI generates radical $\left([\mathrm{M}]^{+\bullet}\right)$ and protonated $\left([\mathrm{M}+\mathrm{H}]^{+}\right)$ions in the source region that are introduced to 
the TIMS-FT-ICR MS via a $0.6 \mathrm{~mm}$ inner diameter, single-bore resistive glass capillary tube, allowing the nebulizer to be maintained at ground potential, while the ends of the capillary can be independently biased. Typical APLI operating conditions were $1 \mathrm{~L} / \mathrm{min}$ dry gas flow rate, 2.1 bar nebulizer gas pressure, and $220^{\circ} \mathrm{C}$ dry gas temperature. FT-ICR MS ion optics were optimized as follows: $-900 \mathrm{~V}$ end-cap source capillary voltage, $180 \mathrm{~V}$ end-cap TIMS capillary voltage, $5 \mathrm{kHz} 400$ peak-to-peak voltage (Vpp) segmented hexapole, 2 kHz $1900 \mathrm{Vpp}$ collision cell, and 4 kHz $400 \mathrm{Vpp}$ ion guide transfer line. The FT-ICR MS experiments were performed by coadding 20016 Megaword (8 s) transients, which were zero-filled to 32Megaword, processed using a half -sine apodization followed by fast-Fourier transform (FFT) and broadband phase correction (absorption spectra using absorption mode processing, AMP); an experimental MS resolving power with AMP at $\mathrm{m} / \mathrm{z} 400$ of $\sim 2,000,000$ was obtained.

\subsubsection{TIMS-FT-ICR MS analysis}

The concept behind TIMS is the use of an electric field to hold ions stationary against a moving gas, so that the drag force is compensated by the electric field and ion

packages are separated across the TIMS analyzer axis based on their mobility. ${ }^{93-95}$ During mobility separation, a quadrupolar field confines the ions in the radial direction to increase trapping efficiency. A simplified schematic of a TIMS analyzer is shown in Appendix 8.1. The mobility, $K$, of an ion in a TIMS cell is described by

$$
K=\frac{v_{8}}{E}=\frac{A}{\left(V_{\text {elution }}-V_{\text {out }}\right)}
$$


where $v_{\mathrm{g}}, E, V_{\text {elution, }}$, and $V_{\text {out }}$ are the velocity of the gas, applied electric field, elution voltage, and tunnel out voltage, respectively. TIMS separation was performed using nitrogen as a bath gas at ca. $300 \mathrm{~K}$, front end $P_{1}=3.0$ and back end $P_{2}=1.1$ mbar pressures, a constant $V_{\text {out }}=35 \mathrm{~V}$ and constant $\mathrm{RF}(840 \mathrm{kHz}$ and $240 \mathrm{Vpp})$ in all electrodes of the entrance funnel, mobility separating section, and exit funnel. Details regarding Oversampling Selected Accumulation TIMS (OSA-TIMS) modes of operation ${ }^{106}$ and specifics compared to traditional TIMS and IMS can be found elsewhere. ${ }^{93-96,98}$ Briefly, OSA-TIMS experiments were performed by scanning $V_{\text {in }}$ from -40 to $-210 \mathrm{~V}$ using a $1 \mathrm{~V}$ ramp size and $0.2 \mathrm{~V}$ increments per step, accumulating 40 mobility experiments per FTICR MS spectrum (4Megaword, 3s transient, with six transients coadded per MS). TIMSFT-ICR MS spectra were processed using sine-squared apodization followed by FFT, in magnitude mode resulting in an experimental MS resolving power of $R \sim 400,000$ at $\mathrm{m} / \mathrm{z}$ 400. Mobility spectra were calibrated using a Tuning Mix calibration standard (Tunemix, G2421A, Agilent Technologies, Santa Clara, CA) with the following reduced mobility $\left(K_{0}\right)$ values $m / z, 622 K_{0}=1.025, m / z, 922 K_{0}=0.840, m / z 1222 K_{0}=0.724, m / z 1522 K_{0}=0.643$ $\mathrm{cm}^{2} \mathrm{~V}^{-1} \mathrm{~s}^{-1}$. Mobilities were correlated with $\operatorname{CCS}(\Omega)$ using the equation

$$
\Omega=\frac{(18 \pi)^{1 / 2}}{16} \frac{z}{\left(k_{\mathrm{B}} T\right)^{1 / 2}}\left[\frac{1}{m_{\mathrm{l}}}+\frac{1}{m_{\mathrm{b}}}\right]^{1 / 2} \frac{1}{K} \frac{760}{P} \frac{T}{273.15} \frac{1}{N^{*}}
$$

where $z$ is the charge of the ion, $k_{\mathrm{B}}$ is the Boltzmann constant, $N^{*}$ is the number density, and $m_{\mathrm{I}}$ and $m_{\mathrm{b}}$ refer to the masses of the ion and bath gas, respectively. ${ }^{121}$ Under these conditions, the experimental TIMS resolving power for Tuning Mix $(\mathrm{m} / \mathrm{z} 622-1522)$ was $\sim 100-250$ as determined by eq 3 . 


$$
R=\frac{\Omega}{\Delta \Omega}
$$

\subsubsection{Data Processing}

FT-ICR MS spectra were externally mass calibrated using the Tuning Mix standard. A peak list was generated allowing a signal-to-noise ratio of 6 , and the data were internally recalibrated (postacquisition) using a double bond equivalence of $9 \mathrm{O}_{1}$ series to improve

overall mass accuracy. ${ }^{122}$ The formulas calculations from the exact mass domain were performed using Composer software (Version 1.0.6, Sierra Analytics, CA) and confirmed with Data Analysis (Bruker Daltonics v 4.2) using formula limits of $\mathrm{C}_{1-100} \mathrm{H}_{1-100} \mathrm{~N}_{0-2} \mathrm{O}_{0-}$ ${ }_{10} \mathrm{~S}_{0-2}$, odd and even electron configurations were allowed, and $\mathrm{M}^{+\cdot}$ and $[\mathrm{M}+\mathrm{H}]^{+}$ion forms. While the oxygen content was limited to ten oxygen atoms in the initial search, molecular matches with more than six oxygen atoms were not observed; we interpret the low number of oxygen content as a consequence of the shorter irradiation time when compared to previously reported experiments. A root-mean-square deviation for the mass assignments of $0.3 \mathrm{ppm}$ was observed. From the generated ion formulas, the double bond equivalence (DBE) was calculated by the equation:

$$
\mathrm{DBE}=\mathrm{C}-\frac{H}{2}+\frac{N}{2}+1
$$

where $C, H$, and $N$ are the number of carbons, hydrogens, and nitrogens in the chemical formula.

The peak list was used for extraction of the ion mobility spectra from the TIMSFT-ICR MS data sets using batch processing in the Data Analysis package (Version v. 4.2, Bruker Daltonics, CA) followed by external mobility calibration using the Tuning Mix 
standards. The TIMS spectrum for each molecular formula was processed using a custombuilt Software Assisted Molecular Elucidation (SAME) package-a specifically designed 2D TIMS-FT-ICR MS data processing script written in Python v2.7. The SAME package utilizes noise removal, mean gap filling, "asymmetric least squares smoothing" baseline correction, ${ }^{70}$ peak detection by continuous wavelet transform (CWT)-based peak detection algorithm (SciPy package), ${ }^{123,} 124$ and Gaussian fitting with nonlinear least-squares functions (Levenberg-Marquardt algorithm). ${ }^{125}$ SAME final outcome is $[\mathrm{m} / \mathrm{z}$; chemical formula; K; CCS] for each 2D TIMS-FT-ICR MS data set. The 2D TIMS-FT-ICR MS contour plots were generated in DataAnalysis (Version v. 4.2, Bruker Daltonics, CA), and all the other plots were generated using matplotlib ${ }^{126}$ and OriginPro 2016 (Originlab Co., MA).

\subsection{Results and Discussion}

The analysis of the WAF samples by APLI-FT-ICR MS can be characterized by a Gaussian-like distribution, centered at $\mathrm{m} / \mathrm{z} 300$ (Figure 8.1). Prior to exposure to light (t0h), 700 peaks were observed in the FT-ICR MS spectra. After the WAF was exposed to light (t-115h), the distribution increased in size, and the center shifted to $m / z 500$, resulting in $\sim 12,000$ peaks, which represents an $\sim 17$-fold increase relative to the unexposed WAF $(\mathrm{t}-0 \mathrm{~h})$. The change in the MS distribution suggests an increased partitioning of the oil in the WAF (e.g., photosolubilization) as well as potential chemical transformations within the WAF as a function of the exposure to light. The use of an ultrahigh resolution mass analyzer allowed the assignment of chemical formulas and to track changes in the WAF as 

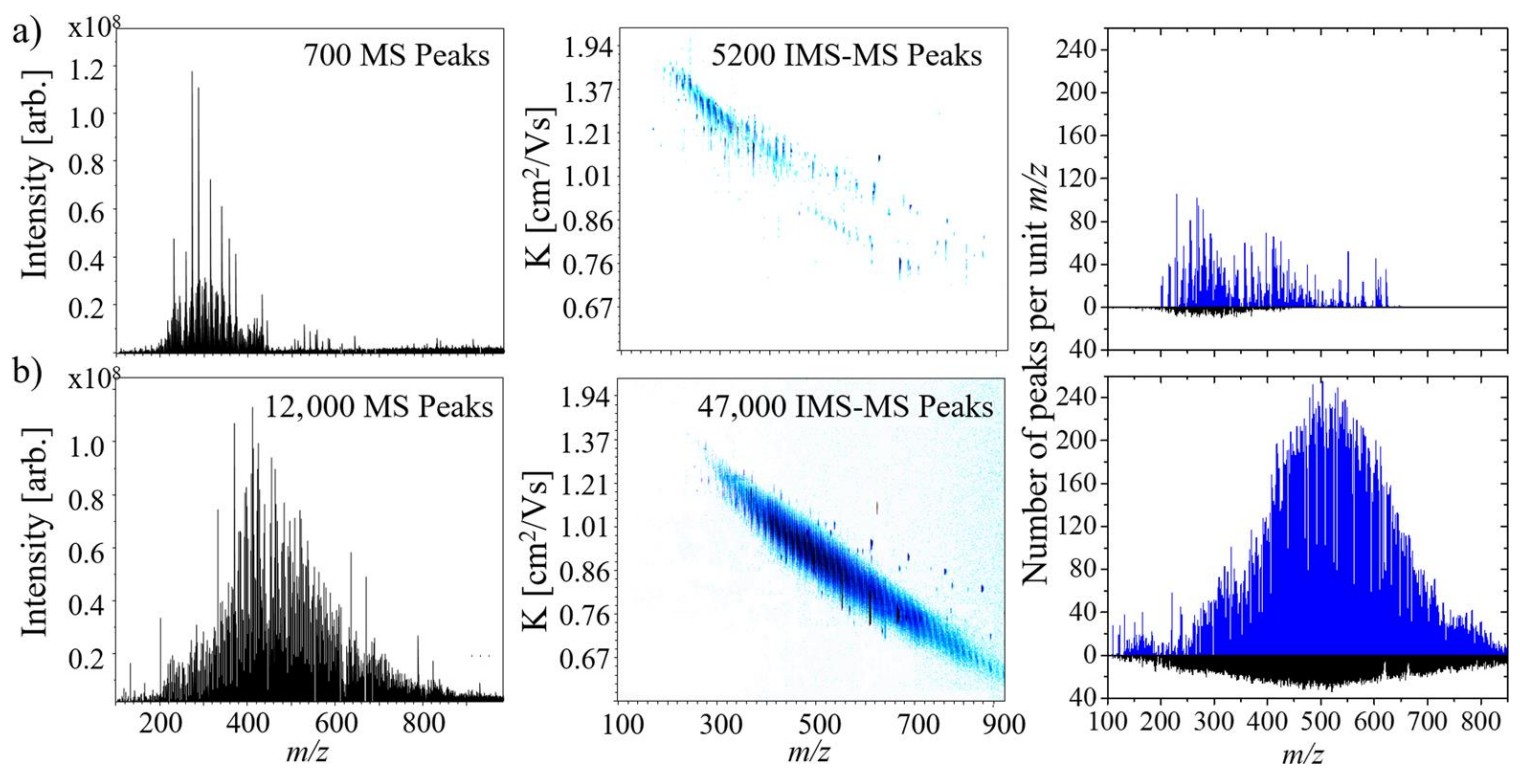

Figure 8.1. Typical FT-ICR MS spectrum and 2D TIMS-FT-ICR MS contour plot for the WAF (a) at 0 and (b) $115 \mathrm{~h}$ light exposed. The number of peaks identified per unit $\mathrm{m} / \mathrm{z}$ in the MS domain (black) and TIMSMS (blue) domains are also shown for (a) and (b). Notice the significant differences in the number of identifications between (a) and (b), as well as between the MS and TIMS MS experiments, increasing the level of molecular features identified per analysis.

a function of the exposure to light (see Appendix 8.2-8.4). By using an APLI source there is an intrinsic bias on the type of molecular ions that can be produced and may result in a lower number of potential features identified from the WAF sample. However, closer inspection to the observed features shows a high chemical diversity and complexity, comparable to those observed using other ionization sources. ${ }^{41} \mathrm{~A}$ follow up analysis using OSA-TIMS in tandem with FT-ICR MS enabled further molecular separation of the WAF content by their mobility (isomeric content) followed by ultrahigh resolution mass analysis. For example, the number of molecular features increased from $\sim 700$ to $\sim 5.2 \mathrm{k}$ and from $\sim 12 \mathrm{k}$ to $\sim 47 \mathrm{k}$ for the $\mathrm{t}-0 \mathrm{~h}$ and $\mathrm{t}-115 \mathrm{~h}$ WAF samples, respectively, by adding the TIMS separation to the FT-ICR MS analysis. Moreover, the isomeric content of the WAF samples is such that TIMS separation increases the number of features up to 6-fold at the nominal level (see Figure 8.1). That is, OSA-TIMS in tandem with FT-ICR MS enabled a more 
a)
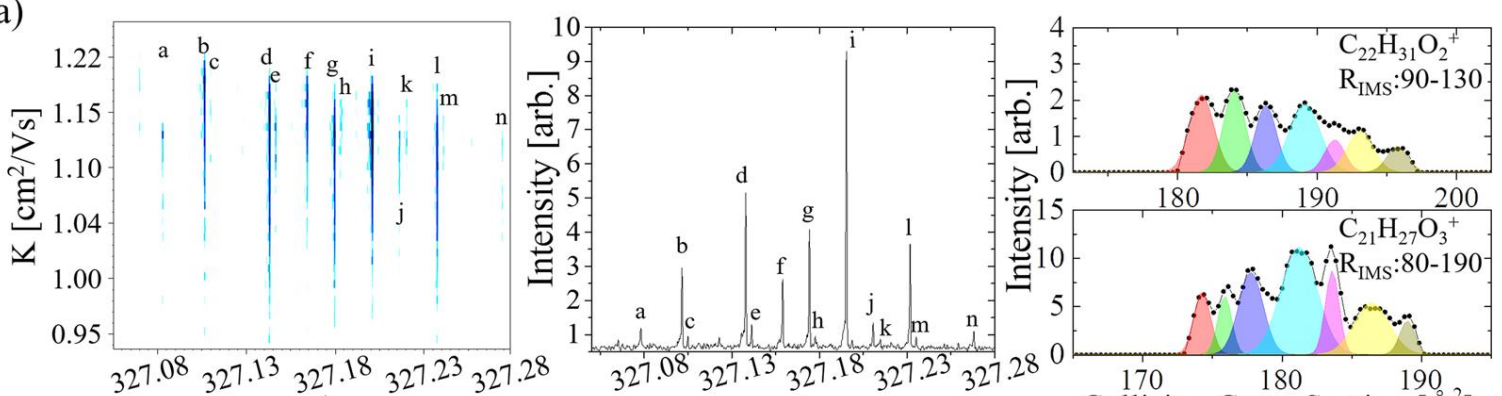

b)

$m / z$
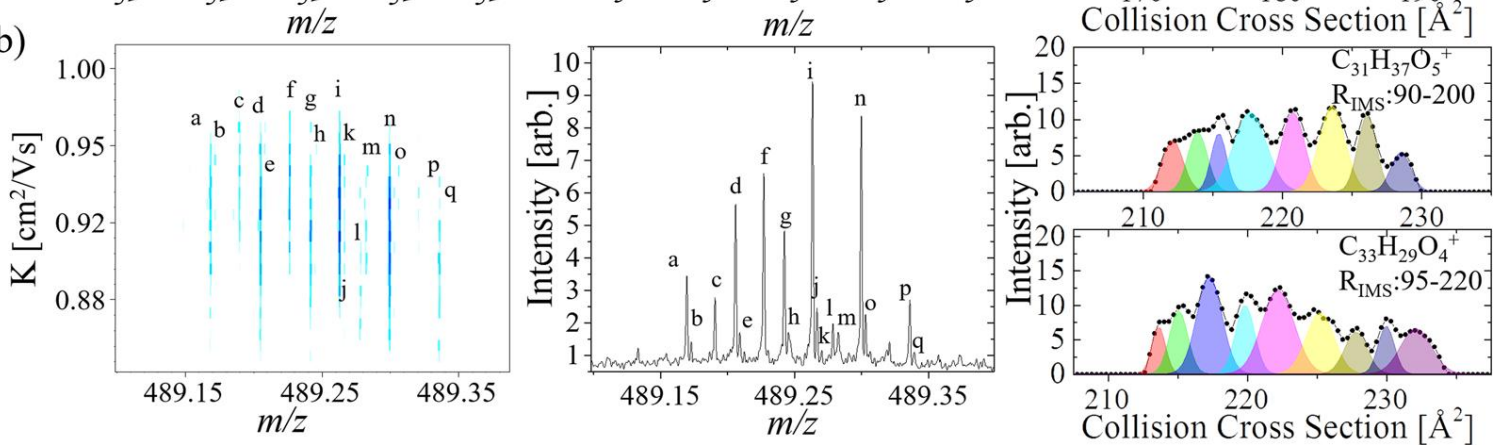

Figure 8.2. Typical 2D TIMS-FT-ICR MS contour plot and MS projections for $m / z$ a) 327 and b) 489 . IMS projections for specific chemical formulas (connected scatterplot) with the unsupervised fitting by the SAME package of minimum number of mobility features is shown. Note that the SAME package relies on the experimental profile of the distribution, which is able to show multiple features due to the high resolving power of the TIMS analyzer. Formula assignments are provided in Table 8.1.

comprehensive analysis of the WAF content by increasing the peak capacity of the analysis using complementary, orthogonal TIMS and FT-ICR MS separations.

Inspection of the 2D-TIMS-FT-ICR MS contour plots indicated that the observed chemical species from the WAF samples using the APLI source are mostly condensed/aromatic molecules (see more details on interpreting 2D-IMS-MS contour plots in ref 106). This observation is consistent with previous analysis. Closer inspection of the 2D TIMS-FT-ICR MS WAF data reveals the spectral complexity in both the mass and mobility dimensions. Particularly, the presence of multiple isobaric interferences (e.g., $-\mathrm{C}_{3}$ to $-\mathrm{SH}_{4}$ splits, $\Delta m=3.4 \mathrm{mDa}$, requiring $\sim 150,000$ resolving power at $\mathrm{m} / \mathrm{z} 489$ ), such as $\mathrm{C}_{31} \mathrm{H}_{37} \mathrm{O}^{+}, \mathrm{C}_{28} \mathrm{H}_{41} \mathrm{OS}^{+}, \mathrm{C}_{25} \mathrm{H}_{45} \mathrm{OS}_{2}{ }^{+}$, as well as multiband ion mobility projections confirm the need for high resolution TIMS analysis during the study of the WAF samples (TIMS 
Table 8.1. Table of Identified Ion Formulas for $m / z, 327$ and 489 as found in Figure 8.2.

\begin{tabular}{|c|c|c|c|c|}
\hline$m / z$ & & $\exp . m / z$ & ion formula & error (ppb) \\
\hline \multirow[t]{14}{*}{327} & a & 327.078031 & $\mathrm{C}_{15} \mathrm{H}_{21} \mathrm{NOS}_{3}{ }^{+}$ & -159 \\
\hline & b & 327.101557 & $\mathrm{C}_{22} \mathrm{H}_{15} \mathrm{O}_{3}^{+}$ & 43 \\
\hline & c & 327.104917 & $\mathrm{C}_{19} \mathrm{H}_{19} \mathrm{O}_{3} \mathrm{~S}^{+}$ & 76 \\
\hline & d & 327.137957 & $\mathrm{C}_{23} \mathrm{H}_{19} \mathrm{O}_{2}$ & -3 \\
\hline & e & 327.141345 & $\mathrm{C}_{20} \mathrm{H}_{23} \mathrm{O}_{2} \mathrm{~S}^{+}$ & -55 \\
\hline & $\mathrm{f}$ & 327.159052 & $\mathrm{C}_{20} \mathrm{H}_{23} \mathrm{O}_{4}^{+}$ & 104 \\
\hline & $\mathrm{g}$ & 327.174325 & $\mathrm{C}_{24} \mathrm{H}_{23} \mathrm{O}^{+}$ & 52 \\
\hline & $\mathrm{h}$ & 327.177623 & $\mathrm{C}_{21} \mathrm{H}_{27} \mathrm{OS}^{+}$ & 275 \\
\hline & i & 327.195425 & $\mathrm{C}_{21} \mathrm{H}_{27} \mathrm{O}_{3}^{+}$ & 141 \\
\hline & j & 327.210673 & $\mathrm{C}_{25} \mathrm{H}_{27}{ }^{+}$ & 165 \\
\hline & $\mathrm{k}$ & 327.214774 & $\mathrm{C}_{22} \mathrm{H}_{31} \mathrm{~S}^{+}$ & -2066 \\
\hline & 1 & 327.231822 & $\mathrm{C}_{22} \mathrm{H}_{31} \mathrm{O}_{2}^{+}$ & 107 \\
\hline & $\mathrm{m}$ & 327.235269 & $\mathrm{C}_{19} \mathrm{H}_{35} \mathrm{O}_{2} \mathrm{~S}$ & -125 \\
\hline & $\mathrm{n}$ & 327.268202 & $\mathrm{C}_{23} \mathrm{H}_{35} \mathrm{O}^{+}$ & 122 \\
\hline \multirow[t]{17}{*}{489} & a & 489.169661 & $\mathrm{C}_{32} \mathrm{H}_{25} \mathrm{O}_{5}{ }^{+}$ & -22 \\
\hline & b & 489.172941 & $\mathrm{C}_{29} \mathrm{H}_{29} \mathrm{O}_{5} \mathrm{~S}^{+}$ & 164 \\
\hline & c & 489.190592 & $\mathrm{C}_{29} \mathrm{H}_{29} \mathrm{O}_{7}$ & 384 \\
\hline & d & 489.205937 & $\mathrm{C}_{33} \mathrm{H}_{29} \mathrm{O}_{4}^{+}$ & 202 \\
\hline & e & 489.209127 & $\mathrm{C}_{30} \mathrm{H}_{33} \mathrm{O}_{4} \mathrm{~S}^{+}$ & 572 \\
\hline & $\mathrm{f}$ & 489.227101 & $\mathrm{C}_{30} \mathrm{H}_{33} \mathrm{O}_{6}^{+}$ & 131 \\
\hline & $\mathrm{g}$ & 489.242320 & $\mathrm{C}_{34} \mathrm{H}_{33} \mathrm{O}_{3}^{+}$ & 206 \\
\hline & $\mathrm{h}$ & 489.245496 & $\mathrm{C}_{31} \mathrm{H}_{37} \mathrm{O}_{3} \mathrm{~S}^{+}$ & 605 \\
\hline & $\mathrm{i}$ & 489.263445 & $\mathrm{C}_{31} \mathrm{H}_{37} \mathrm{O}_{5}^{+}$ & 217 \\
\hline & j & 489.266855 & $\mathrm{C}_{28} \mathrm{H}_{41} \mathrm{O}_{5} \mathrm{~S}^{+}$ & 137 \\
\hline & $\mathrm{k}$ & 489.270311 & $\mathrm{C}_{25} \mathrm{H}_{45} \mathrm{O}_{5} \mathrm{~S}_{2}^{+}$ & -37 \\
\hline & 1 & 489.278685 & $\mathrm{C}_{35} \mathrm{H}_{37} \mathrm{O}_{2}^{+}$ & 249 \\
\hline & $\mathrm{m}$ & 489.282768 & $\mathrm{C}_{32} \mathrm{H}_{41} \mathrm{O}_{2} \mathrm{~S}^{+}$ & -1206 \\
\hline & $\mathrm{n}$ & 489.299809 & $\mathrm{C}_{32} \mathrm{H}_{41} \mathrm{O}_{4}{ }^{+}$ & 260 \\
\hline & o & 489.302875 & $\mathrm{C}_{29} \mathrm{H}_{45} \mathrm{O}_{4} \mathrm{~S}^{+}$ & 883 \\
\hline & $\mathrm{p}$ & 489.336155 & $\mathrm{C}_{33} \mathrm{H}_{45} \mathrm{O}_{3}{ }^{+}$ & 341 \\
\hline & $q$ & 489.339652 & $\mathrm{C}_{30} \mathrm{H}_{49} \mathrm{O}_{3} \mathrm{~S}^{+}$ & 84 \\
\hline
\end{tabular}

resolving power up to 220 is shown in Figure 8.2). While the SAME package is designed to fit the mobility profiles and to provide a minimum number of IMS peaks, inspection of Figure 8.2 shows that coelution may be observed in the ion mobility dimension due to the high sample complexity; in the case shown, restrictions to the peak width fitting in the SAME package were not imposed in order to better account for the structural flexibility of potential isomers. For example, at $\mathrm{m} / \mathrm{z} 327,14 \mathrm{MS}$ peaks are detected and chemical formulas are assigned (see Table 8.1). For each chemical formula, an extracted ion mobility chromatogram was generated, resulting in 47 peaks with assigned chemical formula, mobility, and CCS. Notice that this detailed separation is only possible due to the high resolving power of the TIMS device, the OSA-TIMS method providing sufficient points across the mobility profile, and the ultrahigh resolution and mass accuracy of the FT-ICR 
MS. Moreover, the processing of the 2D-TIMS-FT-ICR MS data using the SAME package is able to deconvolute the mobility profile in a minimum number of isomers (see examples for $\mathrm{C}_{22} \mathrm{H}_{31} \mathrm{O}_{2}{ }^{+}$and $\mathrm{C}_{21} \mathrm{H}_{27} \mathrm{O}_{3}{ }^{+}$in Figure 8.2). A similar complexity can be observed at $\mathrm{m} / \mathrm{z}$ 489, where 17 peaks are resolved in the FT-ICR MS and 123 peaks in the 2D TIMS-FTICR MS. The time independent nature of the OSA-TIMS analysis permits the acquisition of high mass resolution FT-ICR MS spectra, thus maximizing the analytical potential of both techniques while providing precise collision cross section (less than $1 \%$ variability between replicates). It should be pointed out that these results provide a new reference point for the IMS-MS analysis; commonly accessible IMS-MS platforms are limited to IMS resolving power of $\sim 30-60$ (with instances up to 100) and to TOF MS detectors with MS resolving power up to $60 \mathrm{k}^{83-91}$

Taking advantage of the high mobility resolution and ultrahigh mass separation of TIMS-FT-ICR MS, the WAF [ $\mathrm{m} / \mathrm{z}$; chemical formula; K; CCS] components can be followed as a function of the exposure to light for each chemical class (see example in Figure 8.3). The increase in the number of molecular species from the $\mathrm{HC}$ class as a function of the exposure to light suggests that WAF is initially subject to photosolubilization of the surface slick into the water, in good agreement with previous observations. ${ }^{41}$ Results from the $\mathrm{HC}$ class show that the increase in the DBE is associated with a reduction in the CCS for a given carbon number. For example, for $\mathrm{C}_{34}$ the lowest CCS of $210 \AA^{2}$ corresponds to DBE 14 , while DBE 5 has the greatest CCS of $252 \AA^{2}$. This indicates that the degree of condensation (e.g., rings and double bonds) imposes structural boundaries, reducing the CCS of a molecule. ${ }^{85}, 127$ In addition to the initial 
photosolubilization, as the exposure of the WAF to light increases, a greater number of assignments with carbon numbers greater than 40 are observed, particularly comparing 16 and $115 \mathrm{~h}$. Furthermore, there is an increase in the number of oxygenated classes and a
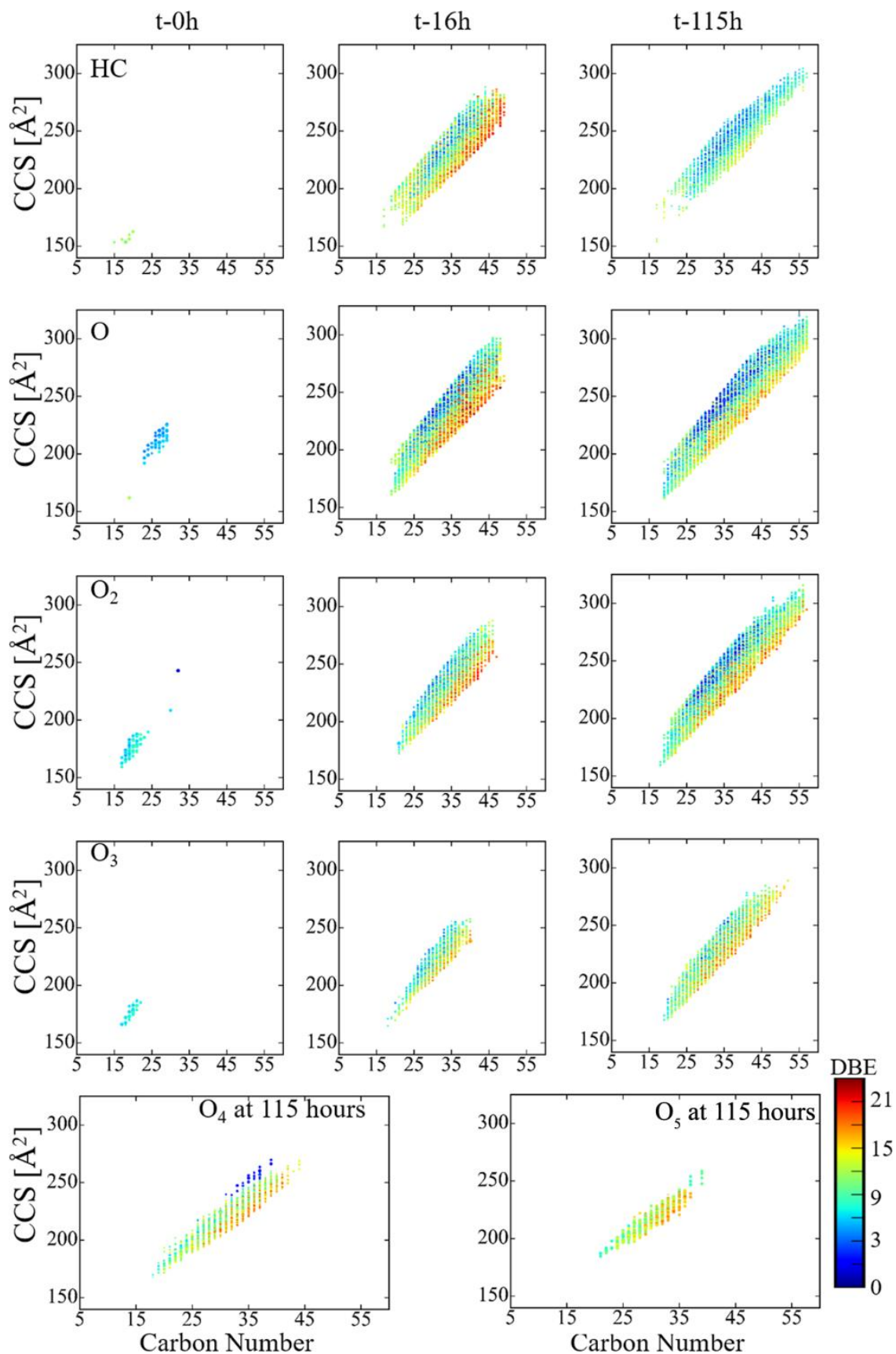

Figure 8.3. Typical size dependence (CCS) with carbon number for the $\mathrm{O}_{0-4}$ chemical classes observed in the WAF samples as a function to exposure to light $(\mathrm{t}-0 \mathrm{~h},-16 \mathrm{~h}$, and $-115 \mathrm{~h})$. The color scale corresponds to the number of rings and double bond equivalents (DBE) of a molecule. Note the large increase in assignments between t-0h and t-16h, as well as increases in oxygen content between t-16h and $\mathrm{t}-115 \mathrm{~h}$. 
decrease in the $\mathrm{HC}$ class, especially for compounds with $\mathrm{DBE}>$ 9. In particular, the identified formulas for the $\mathrm{O}_{4}$ and $\mathrm{O}_{5}$ classes have DBE ranges between 8 and 20, and 9 and 18, and occupy a narrow structural space in the condensed region of the mobility domain, which may indicate that these are products of the higher photosensitive and reactive aromatic HC structures. ${ }^{128}$ Although the WAF transformation mechanisms are not well understood, our results suggest that the phototransformation of the HC molecules in WAF leads to the observation of oxygenated species of the $\mathrm{O}_{4-5}$ class in the first $115 \mathrm{~h}$ of exposure to light.

Changes in the WAF composition can be also followed by the presence of specific chemical formulas as a function of the exposure to light. For example, inspection of the WAF $[\mathrm{m} / z$; chemical formula; $\mathrm{K}$; CCS] components at different time points can be used to infer the degree of chemical transformations (see Figure 8.4). For example, the unexposed WAF, t-0h, has few identifications for the HC class (black bars); however, at t-16h (red bars) there is a significant increase in the number of assignments, 420 new identifications based on MS alone and 3000 based on TIMS-MS followed by a decrease in the number of nonoxygenated molecules (e.g., $\mathrm{HC}$ and $\mathrm{N}$ class molecules) at $\mathrm{t}-115 \mathrm{~h} .{ }^{43}$ This result suggests that there are several chemical transformations occurring as a consequence of the photosolubilization of the surface oil (e.g., indicated by the new identifications for the HC class at t-115h) and increased oxygenation of previously dissolved WAF components (e.g., the 3-fold increase in the number of identifications for the $\mathrm{O}_{3}$ class and the appearance of the $\mathrm{O}_{4}$ and $\mathrm{O}_{5}$ classes with 1140 and 420 identifications, respectively). The oxygenated molecules are also highly isomeric, with up to 9 ion mobility bands per chemical formula. 


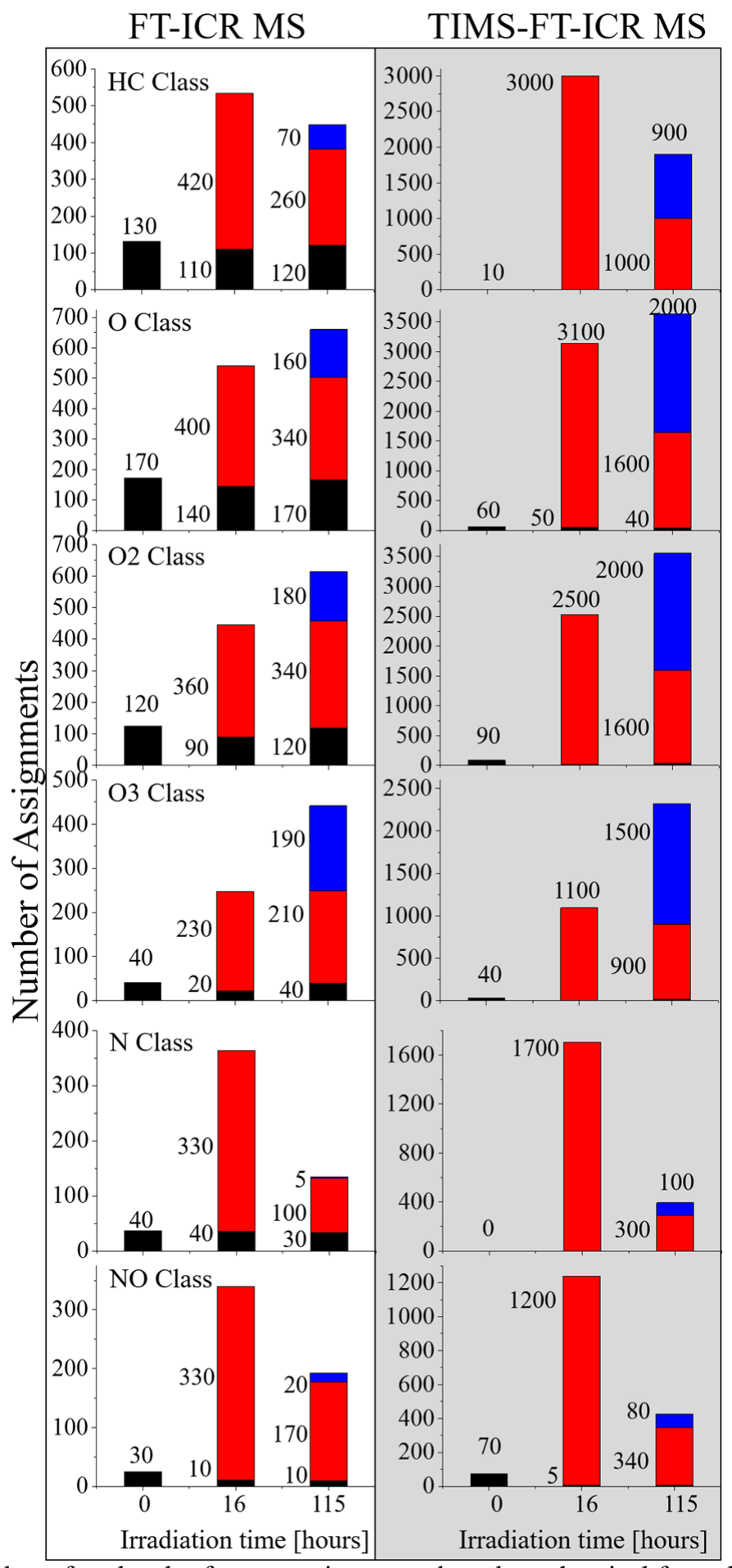

Figure 8.4. Total number of molecular feature assignments based on chemical formula alone from FT-ICR MS measurements and based on chemical formula and IMS profiling from TIMS-FT-ICR MS measurements. Identifications unique to $\mathrm{t}-0, \mathrm{t}-16$, and $\mathrm{t}-115 \mathrm{~h}$ are in black, red, and blue, respectively. When FT-ICR MS is used tracing the evolution of the chemical complexity is incomplete, because the isomeric complexity is not taken into account. Particularly, taking into account the isomeric variability indicates that the composition of the WAF at $\mathrm{t}-115 \mathrm{~h}$ is significantly more complex, and chemically unique, than is observed by FT-ICR MS alone. 
The observation of the $\mathrm{O}_{4-5}$ classes at later irradiation times (t-115h) suggests that these molecules were not originally present in the WAF but are a consequence of the oxygenation process that took place over time, either by the generation of new molecules or by a decrease in matrix effects due to a lower number of UV absorbent molecules. That is, a reduction of molecules that are highly absorbent of the $266 \mathrm{~nm}$ excimer laser may result in greater sensitivity for less absorbent and low concentration molecules. Note that the increase in oxygenation is in good agreement with other MS reports of WAF exposure to light. ${ }^{129,130}$ Small differences were observed between the number of identifications, such as the HC class, by TIMS-MS and MS alone due to the reduced TIMS trapping efficiency for low $\mathrm{m} / \mathrm{z}$ ions and low abundant ions when performing a broad range mobility analysis; however, this limitation can be overcome by performing targeted analysis for smaller PAHs (e.g., naphthalene) as previously reported. ${ }^{96}$

This work showcases the unique advantages of OSA-TIMS in tandem with FT-ICR MS and represents a major step toward the analytical characterization (i.e., high mobility resolving power over 220 combined with ultrahigh mass resolution over 400k) of WAF samples at the molecular level in a single experiment. While these preliminary experiments address one part of a complex environmental challenge (e.g., APLI accessible molecular ions from WAF) and showcase a major improvement in the way we can analytically describe the molecular composition and structural diversity of the WAF, further experiments and developments are needed in order to better understand the environmental implications and the structure-function relationship of the WAF components. For example, a more complete characterization of the WAF content and their transformation products 
and intermediates will require the use of a suite of ionization sources, in both positive and negative polarities, in order to cover a larger range of chemical species during the analysis. $^{62,131}$ Further interpretation of the WAF $[\mathrm{m} / \mathrm{z}$; chemical formula; K; CCS $]$ components can be made with the use of standards, theoretical calculations of candidate structures, ${ }^{79,96,97}$ and the implementation of complementary, postionization MS structural tools in tandem with TIMS-FT-ICR MS (e.g., MS/MS using CID, SID, IRMPD, BIRD, ExD, etc. $)^{132-138}$ Although initial attempts have been made toward the structural characterization, ${ }^{97}$ the 2 D TIMS-FT-ICR MS data sets contain a large amount of analytical data (e.g., over 50k features) in need of further development of theoretical "petroinformatics" and computational approaches capable of producing more detailed structural information on the WAF phototransformation products and intermediates.

\subsection{References}

1.Ghosal, D.; Ghosh, S.; Dutta, T. K.; Ahn, Y. Current state of knowledge in microbial degradation of polycyclic aromatic hydrocarbons (PAHs): a review. Front. Microbiol. 2016, 7, 1369.

2.Kanaly, R. A.; Harayama, S. Biodegradation of high-molecularweight polycyclic aromatic hydrocarbons by bacteria. J. Bacteriol. 2000, 182 (8), 2059-2067.

3.Haritash, A. K.; Kaushik, C. P. Biodegradation aspects of polycyclic aromatic hydrocarbons (PAHs): a review. J. Hazard. Mater. 2009, 169 (1-3), 1-15.

4.King, G. M.; Kostka, J. E.; Hazen, T. C.; Sobecky, P. A. Microbial responses to the Deepwater Horizon oil spill: from coastal wetlands to the deep sea. Ann. Rev. Mar Sci. 2015, 7 (1), 377-401.

5.Venosa, A. D.; Zhu, X. Q. Biodegradation of crude oil contaminating marine shorelines and freshwater wetlands. Spill Sci. Technol. Bull. 2003, 8 (2), 163-178.

6.Wang, J.; Sandoval, K.; Ding, Y.; Stoeckel, D.; Minard-Smith, A.; Andersen, G.; Dubinsky, E. A.; Atlas, R.; Gardinali, P. Biodegradation of dispersed Macondo crude oil 
by indigenous Gulf of Mexico microbial communities. Sci. Total Environ. 2016, 557-558, 453-468.

7.Yang, Z. Y.; Hollebone, B. P.; Wang, Z. D.; Yang, C.; Brown, C.; Zhang, G.; Landriault, M.; Ruan, X. C. A preliminary study for the photolysis behavior of biodiesel and its blends with petroleum oil in simulated freshwater. Fuel 2015, 139, 248-256.

8.Gutierrez, T.; Singleton, D. R.; Berry, D.; Yang, T.; Aitken, M. D.; Teske, A. Hydrocarbon-degrading bacteria enriched by the Deepwater Horizon oil spill identified by cultivation and DNA-SIP. ISME J. 2013, 7 (11), 2091.

9.D’Auria, M.; Emanuele, L.; Racioppi, R.; Velluzzi, V. Photochemical degradation of crude oil: Comparison between direct irradiation, photocatalysis, and photocatalysis on zeolite. J. Hazard. Mater. 2009, 164 (1), 32-8.

10. Sabate, J.; Bayona, J. M.; Solanas, A. M. Photolysis of PAHs in aqueous phase by UV irradiation. Chemosphere 2001, 44 (2), 119-24.

11. Brame, J. A.; Hong, S. W.; Lee, J.; Lee, S. H.; Alvarez, P. J. Photocatalytic pretreatment with food-grade $\mathrm{TiO}(2)$ increases the bioavailability and bioremediation potential of weathered oil from the Deepwater Horizon oil spill in the Gulf of Mexico. Chemosphere 2013, 90 (8), 2315-9.

12. Nicodem, D. E.; Fernandes, M. C. Z.; Guedes, C. L. B.; Correa, R. J. Photochemical processes and the environmental impact of petroleum spills. Biogeochemistry 1997, 39 (2), 121-138.

13. Maki, H.; Sasaki, T.; Harayama, S. Photo-oxidation of biodegraded crude oil and toxicity of the photo-oxidized products. Chemosphere 2001, 44 (5), 1145-51.

14. Lemkau, K. L.; McKenna, A. M.; Podgorski, D. C.; Rodgers, R. P.; Reddy, C. M. Molecular evidence of heavy-oil weathering following the M/V Cosco Busan spill: insights from Fourier transform ion cyclotron resonance mass spectrometry. Environ. Sci. Technol. 2014, 48 (7), 3760-7.

15. Lea-Smith, D. J.; Biller, S. J.; Davey, M. P.; Cotton, C. A.; Sepulveda, B. M. P.; Turchyn, A. V.; Scanlan, D. J.; Smith, A. G.; Chisholm, S. W.; Howe, C. J. Contribution of cyanobacterial alkane production to the ocean hydrocarbon cycle. Proc. Natl. Acad. Sci. U. S.A. 2015, 112 (44), 13591-13596.

16. Valentine, D. L.; Reddy, C. M. Latent hydrocarbons from cyanobacteria. Proc. Natl. Acad. Sci. U. S. A. 2015, 112 (44), 13434-13435.

17. Bacosa, H. P.; Erdner, D. L.; Liu, Z. Differentiating the roles of photooxidation and biodegradation in the weathering of Light Louisiana Sweet crude oil in surface water from the Deepwater Horizon site. Mar. Pollut. Bull. 2015, 95 (1), 265-72. 
18. Lübcke-von Varel, U.; Machala, M.; Ciganek, M.; Neca, J.; Pencikova, K.; Palkova, L.; Vondracek, J.; Löffler, I.; Streck, G.; Reifferscheid, G.; Flückiger-Isler, S.; Weiss, J. M.; Lamoree, M.; Brack, W. Polar Compounds Dominate in Vitro Effects of Sediment Extracts. Environ. Sci. Technol. 2011, 45 (6), 2384-2390.

19. Lee, R. F. Photo-oxidation and photo-toxicity of crude and refined oils. Spill Sci. Technol. Bull. 2003, 8 (2), 157-162.

20. Duesterloh, S.; Short, J. W.; Barron, M. G. Photoenhanced toxicity of weathered Alaska North Slope crude oil to the calanoid copepods Calanus marshallae and Metridia okhotensis. Environ. Sci. Technol. 2002, 36 (18), 3953-9.

21. Barron, M. G.; Carls, M. G.; Heintz, R.; Rice, S. D. Evaluation of fish early life-stage toxicity models of chronic embryonic exposures to complex polycyclic aromatic hydrocarbon mixtures. Toxicol. Sci. 2004, 78 (1), 60-67.

22. Knecht, A. L.; Goodale, B. C.; Truong, L.; Simonich, M. T.; Swanson, A. J.; Matzke, M. M.; Anderson, K. A.; Waters, K. M.; Tanguay, R. L. Comparative developmental toxicity of environmentally relevant oxygenated PAHs. Toxicol. Appl. Pharmacol. 2013, 271 (2), 266-75.

23. Huang, X. D.; McConkey, B. J.; Babu, T. S.; Greenberg, B. M. Mechanisms of photoinduced toxicity of photomodified anthracene to plants: Inhibition of photosynthesis in the aquatic higher plant Lemna gibba (duckweed). Environ. Toxicol. Chem. 1997, 16 (8), 1707-1715.

24. Duxbury, C. L.; Dixon, D. G.; Greenberg, B. M. Effects of simulated solar radiation on the bioaccumulation of polycyclic aromatic hydrocarbons by the duckweed Lemna gibba. Environ. Toxicol. Chem. 1997, 16 (8), 1739-1748.

25. Lampi, M. A.; Gurska, J.; McDonald, K. I.; Xie, F.; Huang, X. D.; Dixon, D. G.; Greenberg, B. M. Photoinduced toxicity of polycyclic aromatic hydrocarbons to Daphnia magna: ultraviolet-mediated effects and the toxicity of polycyclic aromatic hydrocarbon photoproducts. Environ. Toxicol. Chem. 2006, 25 (4), 1079-87.

26. Mason, O. U.; Scott, N. M.; Gonzalez, A.; Robbins-Pianka, A.; Bælum, J.; Kimbrel, J.; Bouskill, N. J.; Prestat, E.; Borglin, S.; Joyner, D. C. Metagenomics reveals sediment microbial community response to Deepwater Horizon oil spill. ISME J. 2014, 8 (7), 1464-1475.

27. Gong, Y.; Zhao, X.; Cai, Z.; O’Reilly, S. E.; Hao, X.; Zhao, D. A review of oil, dispersed oil and sediment interactions in the aquatic environment: Influence on the fate, transport and remediation of oil spills. Mar. Pollut. Bull. 2014, 79 (1-2), 16-33. 
28. Boglaienko, D.; Tansel, B. Partitioning of fresh crude oil between floating, dispersed and sediment phases: Effect of exposure order to dispersant and granular materials. J. Environ. Manage. 2016, 175, 40-45.

29. Sandoval, K.; Ding, Y.; Gardinali, P. Characterization and environmental relevance of oil water preparations of fresh and weathered MC-252 Macondo oils used in toxicology testing. Sci. Total Environ. 2017, 576, 118-128.

30. King, S. M.; Leaf, P. A.; Olson, A. C.; Ray, P. Z.; Tarr, M. A. Photolytic and photocatalytic degradation of surface oil from the Deepwater Horizon spill. Chemosphere 2014, 95 (0), 415-22.

31. Radovic, J. R.; Aeppli, C.; Nelson, R. K.; Jimenez, N.; Reddy, C. M.; Bayona, J. M.; Albaiges, J. Assessment of photochemical processes in marine oil spill fingerprinting. Mar. Pollut. Bull. 2014, 79 (1-2), 268-77.

32. Faksness, L. G.; Altin, D.; Nordtug, T.; Daling, P. S.; Hansen, B.H. Chemical comparison and acute toxicity of water accommodated fraction (WAF) of source and field collected Macondo oils from the Deepwater Horizon spill. Mar. Pollut. Bull. 2015, 91 (1), $222-9$.

33. Theurich, J.; Bahnemann, D. W.; Vogel, R.; Ehamed, F. E.; Alhakimi, G.; Rajab, I. Photocatalytic degradation of naphthalene and anthracene: GC-MS analysis of the degradation pathway. Res. Chem. Intermed. 1997, 23 (3), 247-274.

34. Rontani, J. F.; Giral, P. J. P. Significance of Photosensitized Oxidation of Alkanes during the Photochemical Degradation of Petroleum Hydrocarbon Fractions in Seawater. Int. J. Environ. Anal. Chem. 1990, 42 (1-4), 61-68.

35. Niederer, M. Determination of polycyclic aromatic hydrocarbon and substitutes (nitro-, Oxy-PAHs) in urban soil and airborne particulate by GC-MS and NCI-MS/MS. Environ. Sci. Pollut. Res. 1998, 5 (4), 209-16.

36. Poster, D. L.; Schantz, M. M.; Sander, L. C.; Wise, S. A. Analysis of polycyclic aromatic hydrocarbons (PAHs) in environmental samples: a critical review of gas chromatographic (GC) methods. Anal. Bioanal. Chem. 2006, 386 (4), 859-81.

37. Webb, P. J.; Hamilton, J. F.; Lewis, A. C.; Wirtz, K. Formation of oxygenatedpolycyclic aromatic compounds in aerosol from the photooxidation of o-tolualdehyde. Polycyclic Aromat. Compd. 2006, 26 (4), 237-252.

38. McKenna, A. M.; Nelson, R. K.; Reddy, C. M.; Savory, J. J.; Kaiser, N. K.; Fitzsimmons, J. E.; Marshall, A. G.; Rodgers, R. P. Expansion of the analytical window for oil spill characterization by ultrahigh resolution mass spectrometry: beyond gas chromatography. Environ. Sci. Technol. 2013, 47 (13), 7530-9. 
39. Forth, H. P.; Mitchelmore, C. L.; Morris, J. M.; Lipton, J. Characterization of oil and water accommodated fractions used to conduct aquatic toxicity testing in support of the Deepwater Horizon oil spill natural resource damage assessment. Environ. Toxicol. Chem. 2016.

40. Rowland, S.; Donkin, P.; Smith, E.; Wraige, E. Aromatic Hydrocarbon "Humps" in the Marine Environment: Unrecognized Toxins? Environ. Sci. Technol. 2001, 35 (13), 2640-2644.

41. Ray, P. Z.; Chen, H.; Podgorski, D. C.; McKenna, A. M.; Tarr, M. A. Sunlight creates oxygenated species in water-soluble fractions of Deepwater Horizon oil. J. Hazard. Mater. 2014, 280 (0), 636-43.

42. Ruddy, B. M.; Huettel, M.; Kostka, J. E.; Lobodin, V. V.; Bythell, B. J.; McKenna, A. M.; Aeppli, C.; Reddy, C. M.; Nelson, R. K.; Marshall, A. G.; Rodgers, R. P. Targeted Petroleomics: Analytical Investigation of Macondo Well Oil Oxidation Products from Pensacola Beach. Energy Fuels 2014, 28 (6), 4043-4050.

43. Griffiths, M. T.; Da Campo, R.; O’Connor, P. B.; Barrow, M. P. Throwing light on petroleum: simulated exposure of crude oil to sunlight and characterization using atmospheric pressure photoionization fourier transform ion cyclotron resonance mass spectrometry. Anal. Chem. 2014, 86 (1), 527-34.

44. Islam, A.; Cho, Y.; Yim, U. H.; Shim, W. J.; Kim, Y. H.; Kim, S. The comparison of naturally weathered oil and artificially photodegraded oil at the molecular level by a combination of SARA fractionation and FT-ICR MS. J. Hazard. Mater. 2013, 263 (Part 2), 404-411.

45. Zhu, X.; Shi, Q.; Zhang, Y.; Pan, N.; Xu, C.; Chung, K. H.; Zhao, S. Characterization of Nitrogen Compounds in Coker Heavy Gas Oil and Its Subfractions by Liquid Chromatographic Separation Followed by Fourier Transform Ion Cyclotron Resonance Mass Spectrometry. Energy Fuels 2011, 25, 281.

46. Molnárné Guricza, L.; Schrader, W. Electrospray ionization for determination of non-polar polyaromatic hydrocarbons and polyaromatic heterocycles in heavy crude oil asphaltenes. J. Mass Spectrom. 2015, 50 (3), 549-557.

47. Hourani, N.; Andersson, J. T.; Möller, I.; Amad, M. a.; Witt, M.; Sarathy, S. M. Atmospheric pressure chemical ionization Fourier transform ion cyclotron resonance mass spectrometry for complex thiophenic mixture analysis. Rapid Commun. Mass Spectrom. 2013, 27 (21), 2432-2438.

48. Barrow, M. P.; Peru, K. M.; Headley, J. V. An added dimension: GC atmospheric pressure chemical ionization FTICR MS and the Athabasca oil sands. Anal. Chem. 2014, 86 (16), 8281-8288. 
49. Headley, J. V.; Peru, K. M.; Barrow, M. P. Advances in mass spectrometric characterization of naphthenic acids fraction compounds in oil sands environmental samples and crude oil $\square$ a review. Mass Spectrom. Rev. 2016, 35 (2), 311-328.

50. Headley, J. V.; Peru, K. M.; Mohamed, M. H.; Wilson, L.; McMartin, D. W.; Mapolelo, M. M.; Lobodin, V. V.; Rodgers, R. P.; Marshall, A. G. Atmospheric Pressure Photoionization Fourier Transform Ion Cyclotron Resonance Mass Spectrometry Characterization of Tunable Carbohydrate-Based Materials for Sorption of Oil Sands Naphthenic Acids. Energy Fuels 2014, 28 (3), 1611-1616.

51. Bae, E.; Na, J.-G.; Chung, S. H.; Kim, H. S.; Kim, S. Identification of about 30 000 chemical components in shale oils by electrospray ionization (ESI) and atmospheric pressure photoionization (APPI) coupled with $15 \mathrm{~T}$ Fourier transform ion cyclotron resonance mass spectrometry (FT-ICR MS) and a comparison to conventional oil. Energy Fuels 2010, 24 (4), 2563-2569.

52. Corilo, Y. E.; Rowland, S. M.; Rodgers, R. P. Calculation of the total sulfur content in crude oils by positive-ion atmospheric pressure photoionization Fourier transform ion cyclotron resonance mass spectrometry. Energy Fuels 2016, 30 (5), 3962-3966.

53. Vetere, A.; Schrader, W. Mass Spectrometric Coverage of Complex Mixtures: Exploring the Carbon Space of Crude Oil. ChemistrySelect 2017, 2 (3), 849-853.

54. Stader, C.; Beer, F. T.; Achten, C. Environmental PAH analysis by gas chromatography-atmospheric pressure laser ionization-time of-flight-mass spectrometry (GC-APLI-MS). Anal. Bioanal. Chem. 2013, 405 (22), 7041-7052.

55. Schmitt-Kopplin, P.; Englmann, M.; Rossello-Mora, R.; Schiewek, R.; Brockmann, K. J.; Benter, T.; Schmitz, O. J. Combining chip-ESI with APLI (cESILI) as a multimode source for analysis of complex mixtures with ultrahigh-resolution mass spectrometry. Anal. Bioanal. Chem. 2008, 391 (8), 2803-2809.

56. Schiewek, R.; Schellenträger, M.; Mönnikes, R.; Lorenz, M.; Giese, R.; Brockmann, K.; Gäb, S.; Benter, T.; Schmitz, O. Ultrasensitive determination of polycyclic aromatic compounds with atmospheric-pressure laser ionization as an interface for GC/MS. Anal. Chem. 2007, 79 (11), 4135-4140.

57. Streibel, T.; Zimmermann, R. Resonance-Enhanced Multiphoton Ionization Mass Spectrometry (REMPI-MS): Applications for Process Analysis. Annu. Rev. Anal. Chem. 2014, 7 (1), 361-381.

58. Gaspar, A.; Zellermann, E.; Lababidi, S.; Reece, J.; Schrader, W. Characterization of Saturates, Aromatics, Resins, and Asphaltenes Heavy Crude Oil Fractions by Atmospheric Pressure Laser Ionization Fourier Transform Ion Cyclotron Resonance Mass Spectrometry. Energy Fuels 2012, 26 (6), 3481-3487. 
59. Lababidi, S.; Panda, S. K.; Andersson, J. T.; Schrader, W. Direct coupling of normal-phase high-performance liquid chromatography to atmospheric pressure laser ionization fourier transform ion cyclotron resonance mass spectrometry for the characterization of crude oil. Anal. Chem. 2013, 85 (20), 9478-85.

60. Lababidi, S.; Schrader, W. Online normal-phase high-performance liquid chromatography/Fourier transform ion cyclotron resonance mass spectrometry: effects of different ionization methods on the characterization of highly complex crude oil mixtures. Rapid Commun. Mass Spectrom. 2014, 28 (12), 1345-52.

61. Panda, S. K.; Brockmann, K.-J.; Benter, T.; Schrader, W. Atmospheric pressure laser ionization (APLI) coupled with Fourier transform ion cyclotron resonance mass spectrometry applied to petroleum samples analysis: comparison with electrospray ionization and atmospheric pressure photoionization methods. Rapid Commun. Mass Spectrom. 2011, 25 (16), 2317-2326.

62. Huba, A. K.; Huba, K.; Gardinali, P. R. Understanding the atmospheric pressure ionization of petroleum components: The effects of size, structure, and presence of heteroatoms. Sci. Total Environ. 2016, 568, 1018-1025.

63. Cho, Y.; Ahmed, A.; Islam, A.; Kim, S. Developments in FT-ICR MS instrumentation, ionization techniques, and data interpretation methods for petroleomics. Mass Spectrom. Rev. 2015, 34 (2), 248-263.

64. Pudenzi, M. A.; Eberlin, M. N. Assessing Relative Electrospray Ionization, Atmospheric Pressure Photoionization, Atmospheric Pressure Chemical Ionization, and Atmospheric Pressure Photo- and Chemical Ionization Efficiencies in Mass Spectrometry Petroleomic Analysis via Pools and Pairs of Selected Polar Compound Standards. Energy Fuels 2016, 30 (9), 7125-7133.

65. Benigni, P.; DeBord, J. D.; Thompson, C. J.; Gardinali, P.; Fernandez-Lima, F. Increasing Polyaromatic Hydrocarbon (PAH) Molecular Coverage during Fossil Oil Analysis by Combining Gas Chromatography and Atmospheric-Pressure Laser Ionization Fourier Transform Ion Cyclotron Resonance Mass Spectrometry (FT-ICR MS). Energy Fuels 2016, 30 (1), 196-203.

66. Molnárné Guricza, L.; Schrader, W. New Separation Approach for Asphaltene Investigation: Argentation Chromatography Coupled with Ultrahigh-Resolution Mass Spectrometry. Energy Fuels 2015, 29 (10), 6224-6230.

67. Ghislain, T.; Molnarne Guricza, L.; Schrader, W. Characterization of crude oil asphaltenes by coupling size exclusion chromatography directly to an ultrahigh resolution mass spectrometer. Rapid Commun. Mass Spectrom. 2017, 31 (6), 495-502. 
68. Ramirez, C. E.; Wang, C.; Gardinali, P. R. Fully automated trace level determination of parent and alkylated PAHs in environmental waters by online SPE-LCAPPI-MS/MS. Anal. Bioanal. Chem. 2014, 406 (1), 329-344.

69. Rüger, C. P.; Schwemer, T.; Sklorz, M.; O’Connor, P. B.; Barrow, M. P.; Zimmermann, R. Comprehensive chemical comparison of fuel composition and aerosol particles emitted from a ship diesel engine by gas chromatography atmospheric pressure chemical ionisation ultra-high resolution mass spectrometry with improved data processing routines. Eur. J. Mass Spectrom 2017, 23 (1), 28-39.

70. Vetere, A.; Schrader, W. 1-and 2-Photon ionization for online FAIMS-FTMS coupling allows new insights into the constitution of crude oils. Anal. Chem. 2015, 87 (17), 8874-8879.

71. Ibrahim, Y. M.; Garimella, S. V.; Prost, S. A.; Wojcik, R.; Norheim, R. V.; Baker, E. S.; Rusyn, I.; Smith, R. D. Development of an Ion Mobility SpectrometryOrbitrap Mass Spectrometer Platform. Anal. Chem. 2016, 88 (24), 12152-12160.

72. Kailemia, M. J.; Park, M.; Kaplan, D. A.; Venot, A.; Boons, G. J.; Li, L.; Linhardt, R. J.; Amster, I. J. High-field asymmetric-waveform ion mobility spectrometry and electron detachment dissociation of isobaric mixtures of glycosaminoglycans. J. Am. Soc. Mass Spectrom. 2014, 25 (2), 258-68.

73. Robinson, E. W.; Garcia, D. E.; Leib, R. D.; Williams, E. R. Enhanced mixture analysis of poly(ethylene glycol) using high-field asymmetric waveform ion mobility spectrometry combined with fourier transform ion cyclotron resonance mass spectrometry. Anal. Chem. 2006, 78 (7), 2190-8.

74. Robinson, E. W.; Leib, R. D.; Williams, E. R. The role of conformation on electron capture dissociation of ubiquitin. J. Am. Soc. Mass Spectrom. 2006, 17 (10), 1470-1479.

75. Robinson, E. W.; Sellon, R. E.; Williams, E. R. Peak deconvolution in highfield asymmetric waveform ion mobility spectrometry (FAIMS) to characterize macromolecular conformations. Int. J. Mass Spectrom. 2007, 259 (1-3), 87-95.

76. Robinson, E. W.; Williams, E. R. Multidimensional separations of ubiquitin conformers in the gas phase: relating ion cross sections to H/D exchange measurements. J. Am. Soc. Mass Spectrom. 2005, 16 (9), 1427-37.

77. Tang, X.; Bruce, J. E.; Hill, H. H., Jr. Design and performance of an atmospheric pressure ion mobility Fourier transform ion cyclotron resonance mass spectrometer. Rapid Commun. Mass Spectrom. 2007, 21 (7), 1115-22. 
78. Bluhm, B. K.; Gillig, K. J.; Russell, D. H. Development of a Fourier-transform ion cyclotron resonance mass spectrometer-ion mobility spectrometer. Rev. Sci. Instrum. 2000, 71 (11), 4078-4086.

79. Benigni, P.; Thompson, C. J.; Ridgeway, M. E.; Park, M. A.; Fernandez-Lima, F. Targeted high-resolution ion mobility separation coupled to ultrahigh-resolution mass spectrometry of endocrine disruptors in complex mixtures. Anal. Chem. 2015, 87 (8), 4321-5.

80. Wu, C.; Siems, W. F.; Asbury, G. R.; Hill, H. H. Electrospray Ionization HighResolution Ion Mobility Spectrometry-Mass Spectrometry. Anal. Chem. 1998, 70 (23), 4929-4938.

81. Jarrold, M. F.; Constant, V. A. Silicon cluster ions: evidence for a structural transition. Phys. Rev. Lett. 1991, 67 (21), 2994.

82. Kanu, A. B.; Dwivedi, P.; Tam, M.; Matz, L.; Hill, H. H. Ion mobility-mass spectrometry. J. Mass Spectrom. 2008, 43 (1), 1-22.

83. Becker, C.; Qian, K.; Russell, D. H. Molecular Weight Distributions of Asphaltenes and Deasphaltened Oils Studied by Laser Desorption Ionization and Ion Mobility Mass Spectrometry. Anal. Chem. 2008, 80 (22), 8592-8597.

84. Lalli, P. M.; Corilo, Y. E.; Rowland, S. M.; Marshall, A. G.; Rodgers, R. P. Isomeric Separation and Structural Characterization of Acids in Petroleum by Ion Mobility Mass Spectrometry. Energy Fuels 2015, 29 (6), 3626-3633.

85. Fasciotti, M.; Lalli, P. M.; Klitzke, C. F.; Corilo, Y. E.; Pudenzi, M. A.; Pereira, R. C. L.; Bastos, W.; Daroda, R. J.; Eberlin, M. N. Petroleomics by Traveling Wave Ion Mobility-Mass Spectrometry Using CO2 as a Drift Gas. Energy Fuels 2013, 27 (12), 7277-7286.

86. Fernandez-Lima, F. A.; Becker, C.; McKenna, A. M.; Rodgers, R. P.; Marshall, A. G.; Russell, D. H. Petroleum crude oil characterization by IMS-MS and FTICR MS. Anal. Chem. 2009, 81 (24), 9941-7.

87. Becker, C.; Fernandez-Lima, F. A.; Russell, D. H. Ion Mobility- Mass Spectrometry: A Tool for Characterizing the Petroleome. Spectroscopy 2009, 24 (4), $38-42$.

88. Ahmed, A.; Cho, Y.; Giles, K.; Riches, E.; Lee, J. W.; Kim, H. I.; Choi, C. H.; Kim, S. Elucidating Molecular Structures of Nonalkylated and Short-Chain Alkyl $(\mathrm{n}<5$, $(\mathrm{CH} 2)$ n) Aromatic Compounds in Crude Oils by a Combination of Ion Mobility and Ultrahigh- Resolution Mass Spectrometries and Theoretical Collisional Cross- Section Calculations. Anal. Chem. 2014, 86 (7), 3300-3307. 
89. Ahmed, A.; Cho, Y. J.; No, M.-h.; Koh, J.; Tomczyk, N.; Giles, K.; Yoo, J. S.; Kim, S. Application of the Mason-Schamp Equation and Ion Mobility Mass Spectrometry To Identify Structurally Related Compounds in Crude Oil. Anal. Chem. 2011, 83 (1), 77-83.

90. Farenc, M.; Corilo, Y. E.; Lalli, P. M.; Riches, E.; Rodgers, R. P.; Afonso, C.; Giusti, P. Comparison of Atmospheric Pressure Ionization for the Analysis of Heavy Petroleum Fractions with Ion Mobility-Mass Spectrometry. Energy Fuels 2016, 30 (11), 8896-8903.

91. Santos, J. M.; Galaverna, R. d. S.; Pudenzi, M. A.; Schmidt, E. M.; Sanders, N. L.; Kurulugama, R. T.; Mordehai, A.; Stafford, G. C.; Wisniewski, A.; Eberlin, M. N. Petroleomics by ion mobility mass spectrometry: resolution and characterization of contaminants and additives in crude oils and petrofuels. Anal. Methods 2015, 7 (11), 4450-4463.

92. Lalli, P. M.; Jarvis, J. M.; Marshall, A. G.; Rodgers, R. P. Functional Isomers in Petroleum Emulsion Interfacial Material Revealed by Ion Mobility Mass Spectrometry and Collision-Induced Dissociation. Energy Fuels 2017, 31 (1), 311-318.

93. Hernandez, D. R.; Debord, J. D.; Ridgeway, M. E.; Kaplan, D. A.; Park, M. A.; Fernandez-Lima, F. Ion dynamics in a trapped ion mobility spectrometer. Analyst 2014, 139 (8), 1913-21.

94. Fernandez-Lima, F. A.; Kaplan, D. A.; Park, M. A. Note: Integration of trapped ion mobility spectrometry with mass spectrometry. Rev. Sci. Instrum. 2011, 82 (12), 126106.

95. Fernandez-Lima, F.; Kaplan, D. A.; Suetering, J.; Park, M. A. Gas-phase separation using a trapped ion mobility spectrometer. Int. J. Ion Mobility Spectrom. 2011, 14 (2-3), 93-98.

96. Castellanos, A.; Benigni, P.; Hernandez, D. R.; DeBord, J. D.; Ridgeway, M. E.; Park, M. A.; Fernandez-Lima, F. Fast Screening of Polycyclic Aromatic Hydrocarbons using Trapped Ion Mobility Spectrometry - Mass Spectrometry. Anal. Methods 2014, 6 (23), 9328-9332.

97. Benigni, P.; Marin, R.; Fernandez-Lima, F. Towards unsupervised polyaromatic hydrocarbons structural assignment from SA-TIMS-FTMS data. Int. J. Ion Mobility Spectrom. 2015, 18 (3), 151-157.

98. Schenk, E. R.; Mendez, V.; Landrum, J. T.; Ridgeway, M. E.; Park, M. A.; Fernandez-Lima, F. Direct observation of differences of carotenoid polyene chain cis/trans isomers resulting from structural topology. Anal. Chem. 2014, 86 (4), 2019-24. 
99. Schenk, E. R.; Ridgeway, M. E.; Park, M. A.; Leng, F.; Fernandez-Lima, F. Isomerization kinetics of AT hook decapeptide solution structures. Anal. Chem. 2014, 86 (2), 1210-4.

100. Schenk, E. R.; Nau, F.; Fernandez-Lima, F. Theoretical predictor for candidate structure assignment from IMS data of biomolecule-related conformational space. Int. J. Ion Mobility Spectrom.2015, 18 (1), 23-29.

101. Schenk, E. R.; Almeida, R.; Miksovska, J.; Ridgeway, M. E.; Park, M. A.; Fernandez-Lima, F. Kinetic intermediates of holo- and apo-myoglobin studied using HDXTIMS-MS and molecular dynamic simulations. J. Am. Soc. Mass Spectrom. 2015, 26 (4), 555-63.

102. Benigni, P.; Marin, R.; Molano-Arevalo, J. C.; Garabedian, A.; Wolff, J. J.; Ridgeway, M. E.; Park, M. A.; Fernandez-Lima, F. Towards the Analysis of High Molecular Weight Proteins and Protein complexes using TIMS-MS. Int. J. Ion Mobility Spectrom. 2016, 19 (2), 95-104.

103. Garabedian, A.; Butcher, D.; Lippens, J. L.; Miksovska, J.; Chapagain, P. P.; Fabris, D.; Ridgeway, M. E.; Park, M. A.; Fernandez-Lima, F. Structures of the kinetically trapped i-motif DNA intermediates. Phys. Chem. Chem. Phys. 2016, 18 (38), 26691-26702.

104. Molano-Arevalo, J. C.; Hernandez, D. R.; Gonzalez, W. G.; Miksovska, J.; Ridgeway, M. E.; Park, M. A.; Fernandez-Lima, F. Flavin adenine dinucleotide structural motifs: from solution to gas phase. Anal. Chem. 2014, 86 (20), 10223-30.

105. McKenzie-Coe, A.; DeBord, J. D.; Ridgeway, M.; Park, M.; Eiceman, G.; Fernandez-Lima, F. Lifetimes and stabilities of familiar explosive molecular adduct complexes during ion mobility measurements. Analyst 2015, 140 (16), 5692-9.

106. Benigni, P.; Fernandez-Lima, F. Oversampling Selective Accumulation Trapped Ion Mobility Spectrometry Coupled to FTICR MS: Fundamentals and Applications. Anal. Chem. 2016, 88 (14), 7404-12.

107. Adams, K. J.; Montero, D.; Aga, D.; Fernandez-Lima, F. Isomer separation of polybrominated diphenyl ether metabolites using nanoESI-TIMS-MS. Int. J. Ion Mobility Spectrom. 2016, 19 (2), 69-76.

108. Silveira, J. A.; Ridgeway, M. E.; Laukien, F. H.; Mann, M.; Park, M. A. Parallel accumulation for $100 \%$ duty cycle trapped ion mobility mass spectrometry. Int. J. Mass Spectrom. 2017, 413, 168-175.

109. Michelmann, K.; Silveira, J. A.; Ridgeway, M. E.; Park, M. A. Fundamentals of Trapped Ion Mobility Spectrometry. J. Am. Soc. Mass Spectrom. 2015, 26 (1), 14-24. 
110. Silveira, J. A.; Michelmann, K.; Ridgeway, M. E.; Park, M. A. Fundamentals of Trapped Ion Mobility Spectrometry Part II: Fluid Dynamics. J. Am. Soc. Mass Spectrom. 2016, 27 (4), 585-595.

111. Silveira, J. A.; Danielson, W.; Ridgeway, M. E.; Park, M. A. Altering the mobility-time continuum: nonlinear scan functions for targeted high resolution trapped ion mobility-mass spectrometry. Int. J. Ion Mobility Spectrom. 2016, 19 (2), 87-94.

112. Ridgeway, M. E.; Silveira, J. A.; Meier, J. E.; Park, M. A. Microheterogeneity within conformational states of ubiquitin revealed by high resolution trapped ion mobility spectrometry. Analyst 2015, 140 (20), 6964-6972.

113. Meier, F.; Beck, S.; Grassl, N.; Lubeck, M.; Park, M. A.; Raether, O.; Mann, M. Parallel Accumulation-Serial Fragmentation (PASEF): Multiplying Sequencing Speed and Sensitivity by Synchronized Scans in a Trapped Ion Mobility Device. J. Proteome Res. 2015, 14 (12), 5378-5387.

114. Silveira, J. A.; Ridgeway, M. E.; Park, M. A. High Resolution Trapped Ion Mobility Spectrometery of Peptides. Anal. Chem. 2014, 86 (12), 5624-5627.

115. Ridgeway, M. E.; Wolff, J. J.; Silveira, J. A.; Lin, C.; Costello, C. E.; Park, M. A. Gated trapped ion mobility spectrometry coupled to fourier transform ion cyclotron resonance mass spectrometry. Int. J. Ion Mobility Spectrom. 2016, 19 (2), 77-85.

116. Pu, Y.; Ridgeway, M. E.; Glaskin, R. S.; Park, M. A.; Costello, C. E.; Lin, C. Separation and Identification of Isomeric Glycans by Selected Accumulation-Trapped Ion Mobility Spectrometry-Electron Activated Dissociation Tandem Mass Spectrometry. Anal. Chem. 2016, 88 (7), 3440-3443.

117.Liu, F. C.; Kirk, S. R.; Bleiholder, C. On the structural denaturation of biological analytes in trapped ion mobility spectrometry-mass spectrometry. Analyst 2016, 141 (12), 3722-3730.

118. Lundstedt, S.; White, P. A.; Lemieux, C. L.; Lynes, K. D.; Lambert, L. B.; Oberg, L.; Haglund, P.; Tysklind, M. Sources, fate, and toxic hazards of oxygenated polycyclic aromatic hydrocarbons (PAHs) at PAH-contaminated sites. Ambio 2007, 36 (6), $475-485$.

119. Singer, M. M.; Aurand, D.; Bragin, G. E.; Clark, J. R.; Coelho, G. M.; Sowby, M. L.; Tjeerdema, R. S. Standardization of the preparation and quantitation of wateraccommodated fractions of petroleum for toxicity testing. Mar. Pollut. Bull. 2000, 40 (11), 1007-1016.

120. Benigni, P.; Marin, R.; Sandoval, K.; Gardinali, P.; Fernandez-Lima, F. Chemical Analysis of Water-accommodated Fractions of Crude Oil Spills Using TIMSFT-ICR MS. J. Visualized Exp. 2017, 121, e55352. 
121. McDaniel, E. W.; Mason, E. A. Mobility and diffusion of ions in gases; John Wiley and Sons, Inc.: New York, New York, 1973; p 381.

122. Savory, J. J.; Kaiser, N. K.; McKenna, A. M.; Xian, F.; Blakney, G. T.; Rodgers, R. P.; Hendrickson, C. L.; Marshall, A. G. Parts-perbillion Fourier transform ion cyclotron resonance mass measurement accuracy with a "walking" calibration equation. Anal. Chem. 2011, 83 (5), 1732-1736.

123. Du, P.; Kibbe, W. A.; Lin, S. M. Improved peak detection in mass spectrum by incorporating continuous wavelet transform-based pattern matching. Bioinformatics 2006, 22 (17), 2059-2065.

124. Oliphant, T. E. Python for Scientific Computing. Comput. Sci. Eng. 2007, 9 (3), 10-20.

125. Moré, J. J.; Garbow, B. S.; Hillstrom, K. E. User guide for MINPACK-1; CMP00068642; 1980.

126. Hunter, J. D. Matplotlib: A 2D graphics environment. Comput. Sci. Eng. 2007, 9 (3), 90-95.

127. Fasciotti, M.; Lalli, P. M.; Heerdt, G.; Steffen, R. A.; Corilo, Y. E.; de Sá, G. F.; Daroda, R. J.; Reis, F. d. A. M.; Morgon, N. H.; Pereira, R. C. L.; Eberlin, M. N.; Klitzke, C. F. Structure-drift timerelationships in ion mobility mass spectrometry. Int. J. Ion Mobility Spectrom. 2013, 16 (2), 117-132.

128. Dabestani, R.; Ivanov, I. N. A compilation of physical, spectroscopic and photophysical properties of polycyclic aromatic hydrocarbons. Photochem. Photobiol. 1999, 70 (1), 10-34.

129. Chen, H.; Hou, A.; Corilo, Y. E.; Lin, Q.; Lu, J.; Mendelssohn, I. A.; Zhang, R.; Rodgers, R. P.; McKenna, A. M. 4 Years after the Deepwater Horizon Spill: Molecular Transformation of Macondo Well Oil in Louisiana Salt Marsh Sediments Revealed by FTICR Mass Spectrometry. Environ. Sci. Technol. 2016, 50 (17), 9061-9069.

130. Leshuk, T.; Wong, T.; Linley, S.; Peru, K. M.; Headley, J. V.; Gu, F. Solar photocatalytic degradation of naphthenic acids in oil sands process-affected water. Chemosphere 2016, 144, 1854-1861.

131. Huba, A. K.; Gardinali, P. R. Characterization of a crude oil weathering series by ultrahigh-resolution mass spectrometry using multiple ionization modes. Sci. Total Environ. 2016, 563-564, 600-610.

132. van Agthoven, M. A.; Delsuc, M.-A.; Rolando, C. Twodimensional FTICR/MS with IRMPD as fragmentation mode. Int. J. Mass Spectrom. 2011, 306 (2), 196-203. 
133. Laskin, J. Energy and Entropy Effects in Gas-Phase Dissociation of Peptides and Proteins. In Principles of Mass Spectrometry Applied to Biomolecules; John Wiley \& Sons, Inc.: 2006; pp 619-665.

134. Klassen, J. S.; Schnier, P. D.; Williams, E. R. Blackbody infrared radiative dissociation of oligonucleotide anions. J. Am. Soc. Mass Spectrom. 1998, 9 (11), 1117-24.

135. Felitsyn, N.; Kitova, E. N.; Klassen, J. S. Thermal decomposition of a gaseous multiprotein complex studied by blackbody infrared radiative dissociation. Investigating the origin of the asymmetric dissociation behavior. Anal. Chem. 2001, 73 (19), 4647-61.

136. Dunbar, R. C. BIRD (blackbody infrared radiative dissociation): evolution, principles, and applications. Mass Spectrom. Rev. 2004, 23 (2), 127-58.

137. Yan, J.; Zhou, M.; Gilbert, J. D.; Wolff, J. J.; Somogyi, A.; Pedder, R. E.; Quintyn, R. S.; Morrison, L. J.; Easterling, M. L.; Paša-Tolić, L. Surface-induced dissociation (SID) of protein complexes in a hybrid Fourier transform ion cyclotron resonance (FT-ICR) mass spectrometer. Anal. Chem. 2017, 89 (1), 895-901.

138. Qi, Y.; Volmer, D. A. Structural analysis of small to medium sized molecules by mass spectrometry after electron-ion fragmentation (ExD) reactions. Analyst 2016, 141 (3), 794-806. 
APPENDICIES 


\section{Appendix 2.1}

Images of the a) instrumental set up, b) close up of the GC-FT-ICR MS interface, and c) schematic of the interface.
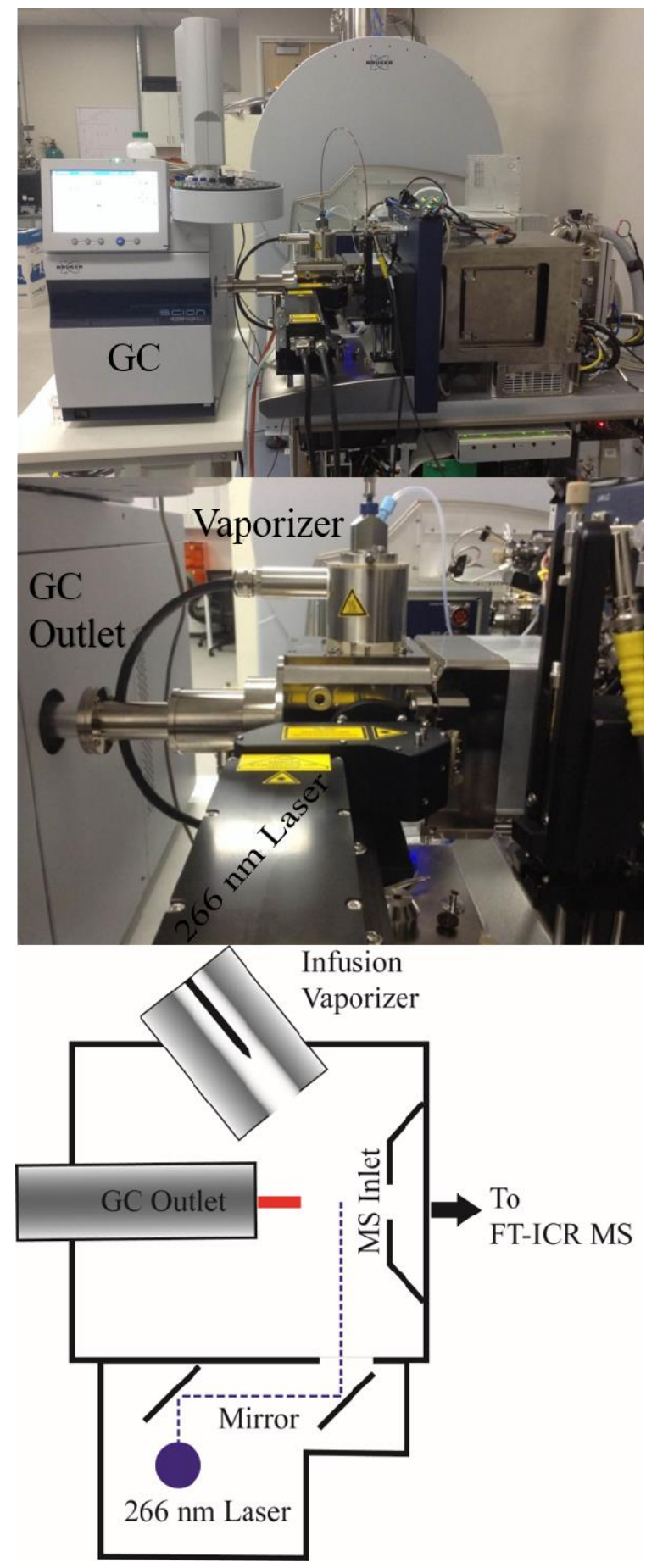


\section{Appendix 2.2}

Combined number of IDs per class for a) Petroleum Crude Oil, b) Organics in Shale, c) Heavy Sweet Crude Oil using GC-APLI-FT-ICR MS and APLI-FT-ICR MS

a)

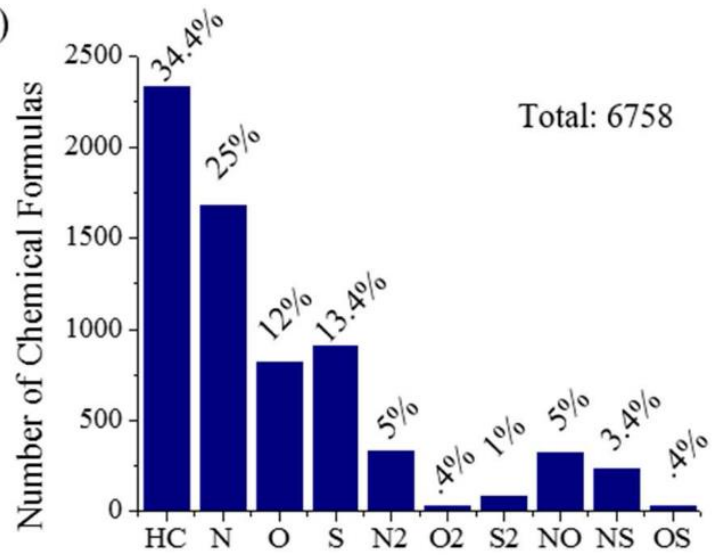

b)

Class
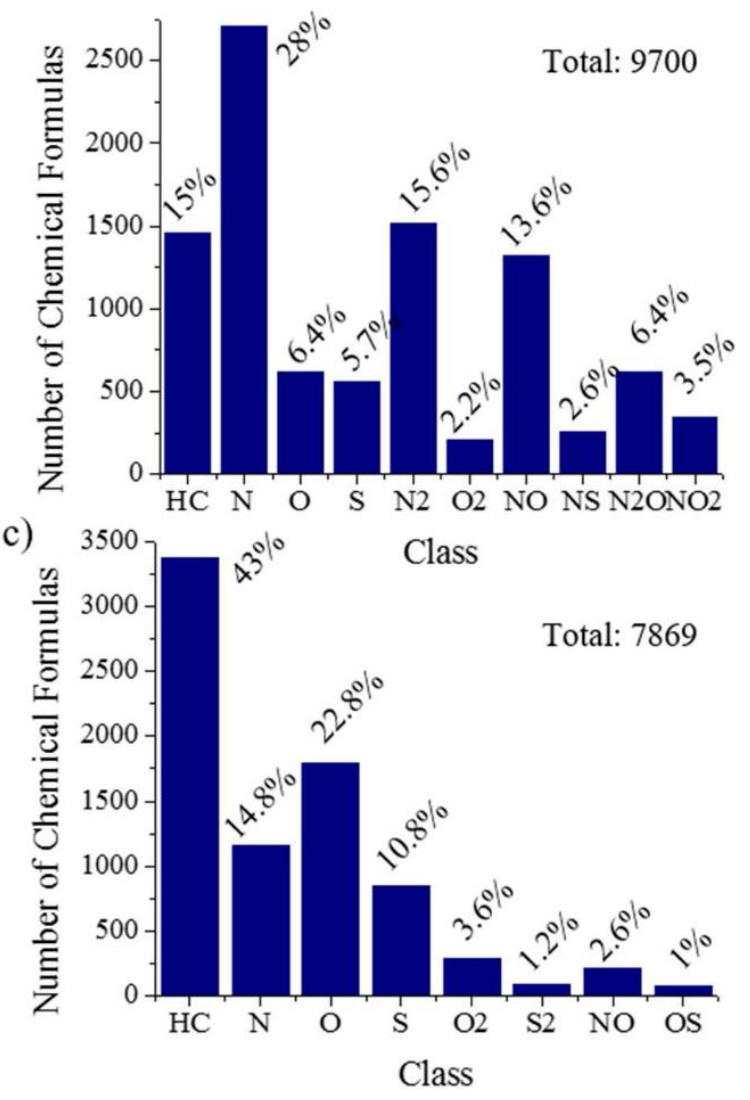


\section{Appendix 2.3}

Comparison of $\mathrm{m} / \mathrm{z} 204$ for the heavy sweet crude oil sample analysed by direct infusion (top, black) and by GC (bottom, red).

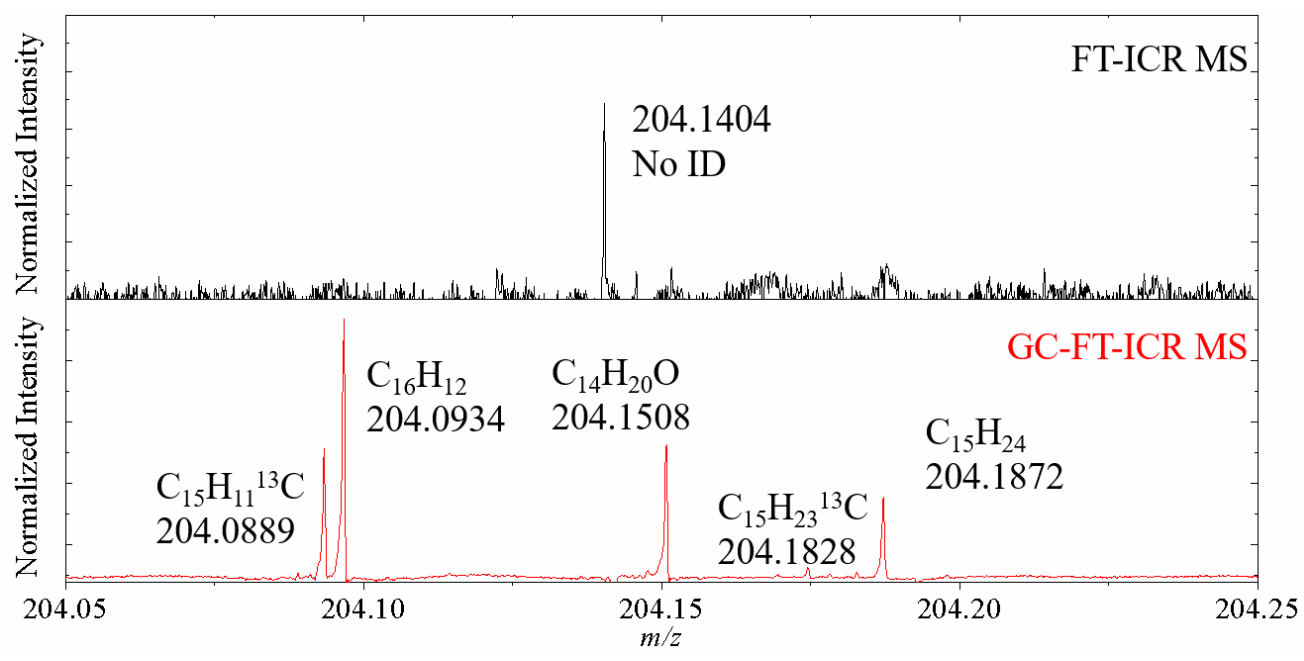




\section{Appendix 2.4}

Matrix suppression experiments for Organics in Shale Oil at a) 1:5, b) 1:10, and c) 1:100 dilution.

a)

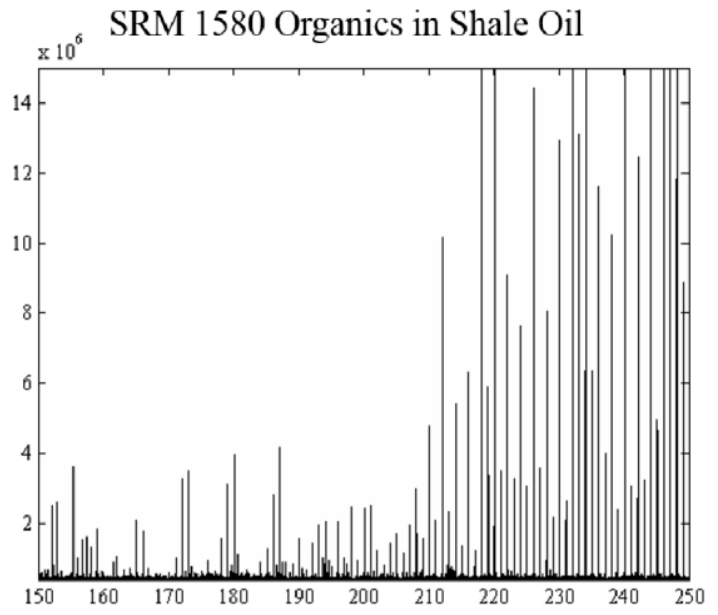

b)

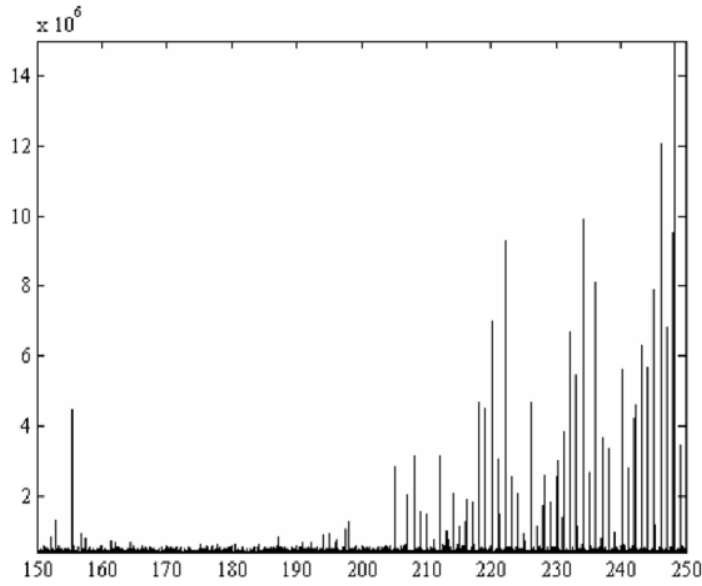

c)

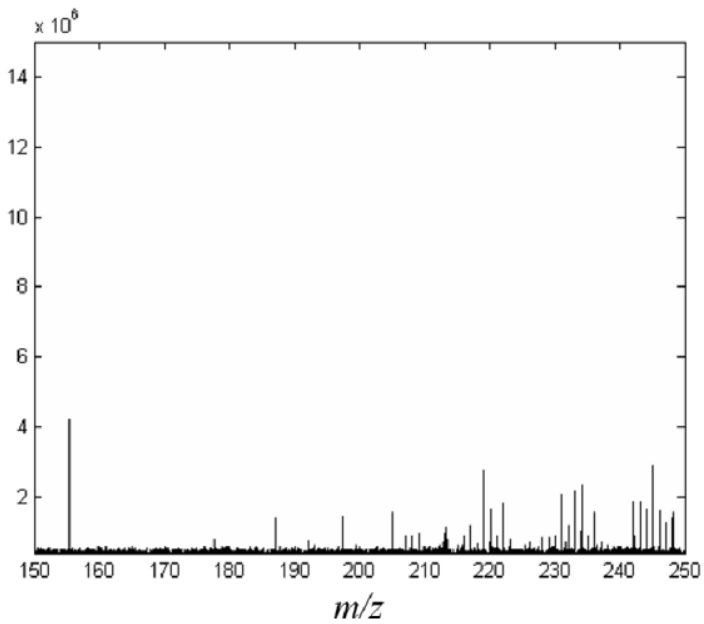




\section{Appendix 2.5}

Matrix suppression experiments for Petroelum Crude Oil at a) 1:5, b) 1:10, and c) 1:100 dilution.

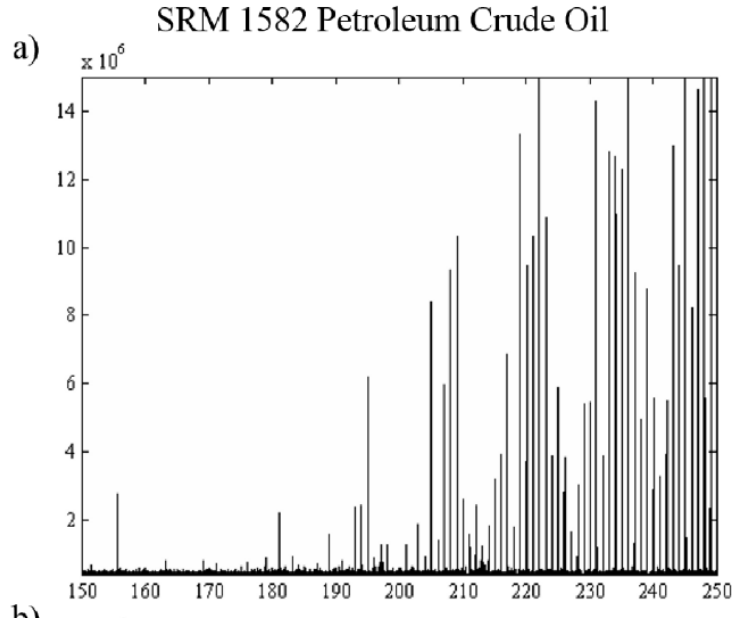

b)

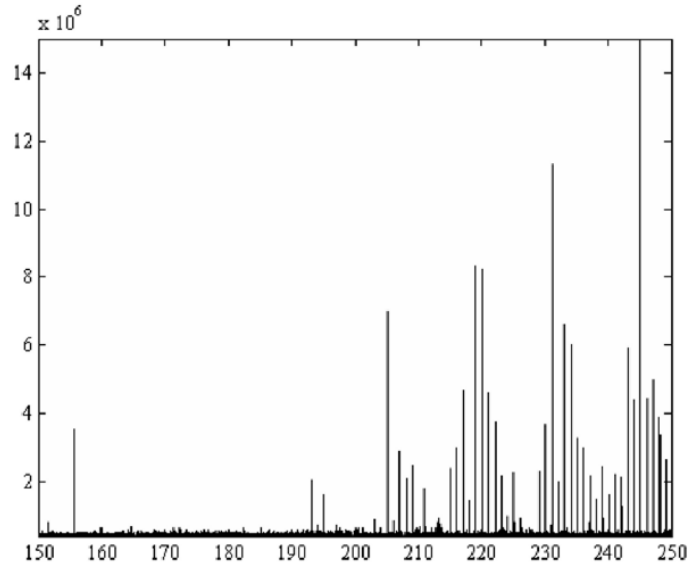

c)

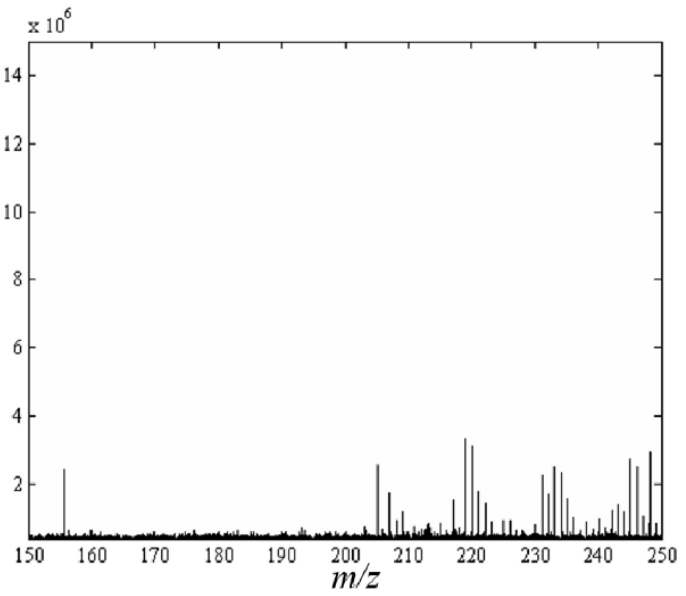




\section{Appendix 2.6}

Assignments and error in assignment for $m / z 178$ and 256 in the Organics in Shale oil GC-APLIFT-ICR MS analysis found in figure 2.4.

\begin{tabular}{|c|c|c|}
\hline $\mathbf{m} / \mathbf{z}$ & Formula & Error (ppm) \\
\hline \multirow{4}{*}{$\stackrel{\infty}{\underline{\Xi}}$} & A $\quad \mathrm{C}_{10} \mathrm{H}_{12} \mathrm{NS}$ & 0.4 \\
\hline & B $\quad \mathrm{C}_{14} \mathrm{H}_{10}$ & -0.21 \\
\hline & $\mathrm{C} \quad \mathrm{C}_{11} \mathrm{H}_{16} \mathrm{NO}$ & 0.03 \\
\hline & D $\quad \mathrm{C}_{12} \mathrm{H}_{20} \mathrm{~N}$ & 0.12 \\
\hline \multirow{12}{*}{ ׁั } & A $\mathrm{C}_{14} \mathrm{H}_{14} \mathrm{~N}_{3} \mathrm{O}_{2}$ & 0.00 \\
\hline & B $\mathrm{C}_{16} \mathrm{H}_{18} \mathrm{NS}$ & -0.78 \\
\hline & $\mathrm{C} \quad \mathrm{C}_{13} \mathrm{H}_{14} \mathrm{~N}_{5} \mathrm{O}$ & 0.16 \\
\hline & D $\mathrm{C}_{20} \mathrm{H}_{16}$ & 0.2 \\
\hline & E $\mathrm{C}_{17} \mathrm{H}_{20} \mathrm{~S}$ & -0.03 \\
\hline & $\mathrm{F} \quad \mathrm{C}_{17} \mathrm{H}_{20} \mathrm{O}_{2}$ & 0.27 \\
\hline & $\mathrm{G} \mathrm{C}_{16} \mathrm{H}_{21} \mathrm{NO}^{13} \mathrm{C}$ & -0.25 \\
\hline & $\mathrm{H} \mathrm{C} \mathrm{C}_{17} \mathrm{H}_{20} \mathrm{NO}$ & 0.0 \\
\hline & I $\quad \mathrm{C}_{18} \mathrm{H}_{24} \mathrm{O}$ & 0.14 \\
\hline & $\mathrm{J} \quad \mathrm{C}_{17} \mathrm{H}_{25} \mathrm{~N}^{13} \mathrm{C}$ & -0.16 \\
\hline & $\mathrm{K} \mathrm{C}_{18} \mathrm{H}_{26} \mathrm{~N}$ & 0.06 \\
\hline & $\mathrm{L} \quad \mathrm{C}_{19} \mathrm{H}_{28}$ & 0.24 \\
\hline
\end{tabular}




\section{Appendix 3.1}

Relative abundance of the analyte in the PAHs in Soil standard as reported by manufacturer. PAHs in Soil. SQC017-40G Mfg Lot 014529

\begin{tabular}{|l|r|c|}
\hline Analyte & $\begin{array}{c}\text { Concentration } \\
\text { In Soil }\end{array}$ & Units \\
\hline Naphthalene 5005 & $906 \pm$ & $\mu \mathrm{g} / \mathrm{Kg}$ \\
\hline Acenaphthene 5500 & $533 \pm$ & $\mu \mathrm{g} / \mathrm{Kg}$ \\
\hline Acenaphthylene 5505 & $236 \pm$ & $\mu \mathrm{g} / \mathrm{Kg}$ \\
\hline Anthracene 5555 & $827 \pm$ & $\mu \mathrm{g} / \mathrm{Kg}$ \\
\hline Benzo(a)Anthracene 5575 & $260 \pm$ & $\mu \mathrm{g} / \mathrm{Kg}$ \\
\hline Benzo(a)Pyrene 5580 & $151 \pm$ & $\mu \mathrm{g} / \mathrm{Kg}$ \\
\hline Benzo(b)Fluoranthene 5585 & $461 \pm$ & $\mu \mathrm{g} / \mathrm{Kg}$ \\
\hline Benzo(g,h,i)perylene 5590 & $262 \pm$ & $\mu \mathrm{g} / \mathrm{Kg}$ \\
\hline Benzo(k)Fluoranthene 5600 & $383 \pm$ & $\mu \mathrm{g} / \mathrm{Kg}$ \\
\hline Chrysene 5855 & $298 \pm$ & $\mu \mathrm{g} / \mathrm{Kg}$ \\
\hline Dibenzo(a,h)Anthracene 5895 & $613 \pm$ & $\mu \mathrm{g} / \mathrm{Kg}$ \\
\hline Fluoranthene 6265 & $314 \pm$ & $\mu \mathrm{g} / \mathrm{Kg}$ \\
\hline Fluorene 6270 & $238 \pm$ & $\mu \mathrm{g} / \mathrm{Kg}$ \\
\hline Indeno(1,2,3-cd) Pyrene 6315 & $845 \pm$ & $\mu \mathrm{g} / \mathrm{Kg}$ \\
\hline Phenanthrene 6615 & $341 \pm$ & $\mu \mathrm{g} / \mathrm{Kg}$ \\
\hline Pyrene 6665 & \\
\hline
\end{tabular}




\section{Appendix 3.2}

Experimental and theoretical ion neutral collision cross section for standard and extract PAHs obtained using TIMS-MS.

\begin{tabular}{|c|c|c|c|c|c|c|}
\hline Analyte & $\mathrm{m} / \mathrm{z}$ & $\begin{array}{l}\text { Experimental } \\
\text { CCS in N2 }\end{array}$ & $\begin{array}{c}\text { Theoretical } \\
\text { CCS in N2 } \\
\text { (T.M.) }\end{array}$ & $\begin{array}{c}\text { Theoretical } \\
\text { CCS in N2 } \\
\text { (T.M.) }\end{array}$ & $\begin{array}{c}\text { Theoretical } \\
\text { CCS in N2 } \\
\text { (T.M.) }\end{array}$ & $\begin{array}{c}\text { Theoretical } \\
\text { CCS in N2 } \\
\text { (T.M.) }\end{array}$ \\
\hline Energy optimization & - & - & $\begin{array}{l}\text { b3lyp/6- } \\
31+g(d)\end{array}$ & $\begin{array}{c}\text { b3lyp/6- } \\
31++g(d, p)\end{array}$ & $\begin{array}{l}\text { b3lyp/cc- } \\
\text { pvdz }\end{array}$ & $\begin{array}{l}\text { b3lyp/cc- } \\
\text { pvtz }\end{array}$ \\
\hline Naphthalene & 128.062 & 114.15 & 119.31 & 119.29 & 119.35 & 120.62 \\
\hline Acenaphthylene & 152.062 & 121.51 & 123.67 & 123.83 & 123.57 & 123.83 \\
\hline Acenaphthene & 154.078 & 123.19 & 125.85 & 125.67 & 125.55 & 125.51 \\
\hline Fluorene & 166.078 & 125.40 & 132.31 & 132.27 & 132.21 & 132.21 \\
\hline Anthracene & 178.078 & 128.18 & 134.30 & 134.15 & 134.05 & 134.20 \\
\hline Phenanthrene & 178.078 & 128.18 & 131.82 & 131.76 & 131.83 & 131.81 \\
\hline Fluoroanthene & 202.078 & 134.62 & 152.04 & 152.02 & 152.10 & 151.92 \\
\hline Pyrene & 202.078 & 134.26 & 136.68 & 136.76 & 136.51 & 136.70 \\
\hline 1,2-Benzanthracene & 228.093 & 143.47 & 149.15 & 149.32 & 149.26 & 148.98 \\
\hline 2,3-Benzanthracene & 228.093 & 142.00 & 148.28 & 148.28 & 148.16 & 148.16 \\
\hline CHRYSENE & 228.093 & 142.34 & 150.61 & 150.57 & 150.56 & 150.68 \\
\hline TRIPHENYLENE & 228.093 & 140.87 & 146.76 & 146.84 & 146.38 & 146.68 \\
\hline Benzo(a)pyrene & 252.093 & 146.20 & 151.19 & 151.21 & 151.63 & 151.58 \\
\hline Benzo(e)pyrene & 252.093 & 146.42 & 148.34 & 148.41 & 148.45 & 148.39 \\
\hline Benzo(b)fluoranthene & 252.093 & 151.78 & 152.04 & 152.06 & 152.09 & 151.92 \\
\hline Benzo(k)fluoranthene & 252.093 & 151.78 & 157.81 & 157.67 & 157.88 & 156.78 \\
\hline Perylene & 252.093 & 146.76 & 152.65 & 152.68 & 152.91 & 152.73 \\
\hline Benzo(g,h,i)perylene & 276.093 & 154.00 & 157.47 & 157.49 & 157.17 & 157.45 \\
\hline $\begin{array}{l}\text { Indeno(1,2,3- } \\
\text { cd)pyrene }\end{array}$ & 276.093 & 154.00 & 160.80 & 161.05 & 160.69 & 160.98 \\
\hline $\begin{array}{c}1,2,3,4- \\
\text { Dibenzanthracene }\end{array}$ & 278.109 & 157.52 & 162.98 & 162.86 & 162.67 & 162.88 \\
\hline $\begin{array}{c}1,2: 5,6- \\
\text { Dibenzanthracene }\end{array}$ & 278.109 & 159.53 & 163.21 & 162.99 & 162.84 & 162.99 \\
\hline Pentacene & 278.109 & 158.02 & 169.45 & 169.44 & 169.50 & 169.50 \\
\hline Rubrene & 532.219 & 232.24 & 240.01 & 239.94 & 240.09 & 239.95 \\
\hline
\end{tabular}




\section{Appendix 3.3}

PAH geometry and partial charges (use for MOBCAL input files) at different DFT/B3LYP levels of theory.

\begin{tabular}{|c|c|c|c|c|c|c|c|}
\hline \multicolumn{8}{|c|}{ Naphthalene } \\
\hline \multicolumn{4}{|c|}{ Atom Orientation } & \multicolumn{4}{|c|}{ Electrostatic Potential Charges } \\
\hline \multicolumn{3}{|c|}{ Coordinates $(\mathrm{x}, \mathrm{y}, \mathrm{z})$} & \multirow{2}{*}{$\begin{array}{c}\text { mass } \\
12\end{array}$} & \multirow{2}{*}{$\begin{array}{c}\text { b3lyp/6- } \\
31+G(d) \\
-0.038951\end{array}$} & \multirow{2}{*}{$\begin{array}{c}\text { b3lyp/6- } \\
31++\mathrm{G}(\mathrm{d}, \mathrm{p})\end{array}$} & \multirow{2}{*}{$\begin{array}{l}\begin{array}{l}\text { b3lyp/cc- } \\
\text { pvdz }\end{array} \\
-0.058944\end{array}$} & \multirow{2}{*}{$\begin{array}{c}\text { b3lyp/cc-pvtz } \\
-0.064265\end{array}$} \\
\hline 0.000000 & 0.695498 & 2.450190 & & & & & \\
\hline 0.000000 & 1.400938 & 1.234971 & 12 & -0.114876 & -0.049845 & -0.021976 & -0.049169 \\
\hline 0.000000 & 0.715353 & 0.000000 & 12 & 0.152010 & 0.080883 & 0.042854 & 0.080191 \\
\hline 0.000000 & -0.715353 & 0.000000 & 12 & 0.152010 & 0.080883 & 0.042854 & 0.080191 \\
\hline 0.000000 & -1.400938 & 1.234971 & 12 & -0.114876 & -0.049845 & -0.021976 & -0.049169 \\
\hline 0.000000 & -0.695498 & 2.450190 & 12 & -0.038951 & -0.069869 & -0.058943 & -0.064265 \\
\hline 0.000000 & 2.485531 & -1.241694 & 1 & 0.170471 & 0.162491 & 0.150744 & 0.160024 \\
\hline 0.000000 & 1.240924 & 3.385968 & 1 & 0.157350 & 0.170148 & 0.160388 & 0.166899 \\
\hline 0.000000 & 2.485531 & 1.241694 & 1 & 0.170471 & 0.160869 & 0.150076 & 0.158070 \\
\hline 0.000000 & 1.400938 & -1.234971 & 12 & -0.114876 & -0.059397 & -0.026816 & -0.059076 \\
\hline 0.000000 & -1.400938 & -1.234971 & 12 & -0.114876 & -0.059397 & -0.026816 & -0.059076 \\
\hline 0.000000 & -2.485531 & 1.241694 & 1 & 0.170471 & 0.160869 & 0.150076 & 0.158070 \\
\hline 0.000000 & -1.240924 & 3.385968 & 1 & 0.157350 & 0.170148 & 0.160388 & 0.166899 \\
\hline 0.000000 & -0.695498 & -2.450190 & 12 & -0.038951 & -0.064252 & -0.056377 & -0.057774 \\
\hline 0.000000 & 0.695498 & -2.450190 & 12 & -0.038951 & -0.064252 & -0.056376 & -0.057774 \\
\hline 0.000000 & -2.485531 & -1.241694 & 1 & 0.170471 & 0.162491 & 0.150744 & 0.160024 \\
\hline 0.000000 & -1.240924 & -3.385968 & 1 & 0.157350 & 0.168972 & 0.160051 & 0.165099 \\
\hline 0.000000 & 1.240924 & -3.385968 & 1 & 0.157350 & 0.168972 & 0.160051 & 0.165099 \\
\hline
\end{tabular}




\begin{tabular}{|c|c|c|c|c|c|c|c|}
\hline \multicolumn{8}{|c|}{ Acenaphthylene } \\
\hline \multicolumn{4}{|c|}{ Atom Orientation } & \multicolumn{4}{|c|}{ Electrostatic Potential Charges } \\
\hline \multicolumn{3}{|c|}{ Coordinates $(\mathrm{x}, \mathrm{y}, \mathrm{z})$} & \multirow{2}{*}{$\begin{array}{r}\text { Mass } \\
12\end{array}$} & \multirow{2}{*}{$\begin{array}{c}\text { b3lyp/6- } \\
31+G(d)\end{array}$} & \multirow{2}{*}{$\begin{array}{c}\text { b3lyp/6- } \\
31++G(d, p \\
) \\
-0.059014\end{array}$} & \multirow{2}{*}{$\begin{array}{c}\begin{array}{c}\text { b3lyp/cc- } \\
\text { pvdz }\end{array} \\
-0.033746\end{array}$} & \multirow{2}{*}{$\begin{array}{r}\text { b3lyp/cc-pvtz } \\
-0.050406\end{array}$} \\
\hline 0.000000 & 0.715947 & -2.301098 & & & & & \\
\hline 0.000000 & 1.328768 & -3.192764 & 1 & 0.184956 & 0.184844 & 0.172211 & 0.18118 \\
\hline 0.000000 & -1.328768 & -3.192764 & 1 & 0.184956 & 0.184844 & 0.172211 & 0.18118 \\
\hline 0.000000 & -0.715947 & -2.301098 & 12 & -0.046704 & -0.059014 & -0.033746 & -0.050406 \\
\hline 0.000000 & 1.179086 & -0.958357 & 12 & 0.034121 & 0.034717 & 0.017241 & 0.026398 \\
\hline 0.000000 & 0.000000 & -0.142030 & 12 & 0.215965 & 0.196254 & 0.159179 & 0.189215 \\
\hline 0.000000 & 2.419985 & -0.322917 & 12 & -0.147334 & -0.116479 & -0.080705 & -0.109691 \\
\hline 0.000000 & 0.000000 & 1.253200 & 12 & 0.103159 & 0.089488 & 0.056932 & 0.091339 \\
\hline 0.000000 & -1.179086 & -0.958357 & 12 & 0.034121 & 0.034717 & 0.017241 & 0.026398 \\
\hline 0.000000 & 2.445342 & 1.086295 & 12 & -0.071846 & -0.08958 & -0.093565 & -0.081264 \\
\hline 0.000000 & 3.347506 & -0.882947 & 1 & 0.183477 & 0.18138 & 0.168408 & 0.17717 \\
\hline 0.000000 & 1.284170 & 1.864151 & 12 & -0.140684 & -0.119056 & -0.077527 & -0.117519 \\
\hline 0.000000 & -1.284170 & 1.864151 & 12 & -0.140684 & -0.119056 & -0.077527 & -0.117519 \\
\hline 0.000000 & -2.419985 & -0.322917 & 12 & -0.147334 & -0.116479 & -0.080705 & -0.109691 \\
\hline 0.000000 & 3.404903 & 1.588594 & 1 & 0.165334 & 0.165423 & 0.158728 & 0.161735 \\
\hline 0.000000 & 1.369823 & 2.945163 & 1 & 0.179118 & 0.174895 & 0.160898 & 0.172119 \\
\hline 0.000000 & -2.445342 & 1.086295 & 12 & -0.071846 & -0.08958 & -0.093565 & -0.081264 \\
\hline 0.000000 & -1.369823 & 2.945163 & 1 & 0.179118 & 0.174895 & 0.160898 & 0.172119 \\
\hline 0.000000 & -3.347506 & -0.882947 & 1 & 0.183477 & 0.18138 & 0.168408 & 0.17717 \\
\hline 0.000000 & -3.404903 & 1.588594 & 1 & 0.165334 & 0.165423 & 0.158728 & 0.161735 \\
\hline
\end{tabular}




\begin{tabular}{|c|c|c|c|c|c|c|c|}
\hline \multicolumn{8}{|c|}{ Acenaphthene } \\
\hline \multicolumn{4}{|c|}{ Atom Orientation } & \multicolumn{4}{|c|}{ Electrostatic Potential Charges } \\
\hline \multicolumn{3}{|c|}{ Coordinates $(\mathrm{x}, \mathrm{y}, \mathrm{z})$} & \multirow{2}{*}{$\begin{array}{c}\text { Mass } \\
12 \\
\end{array}$} & \multirow{2}{*}{$\begin{array}{c}\text { b3lyp/6- } \\
31+G(d) \\
-0.147553\end{array}$} & \multirow{2}{*}{$\begin{array}{c}\text { b3lyp/6- } \\
31++\mathrm{G}(\mathrm{d}, \mathrm{p}) \\
-0.143591\end{array}$} & \multirow{2}{*}{$\begin{array}{c}\begin{array}{c}\text { b3lyp/cc- } \\
\text { pvdz }\end{array} \\
-0.120477\end{array}$} & \multirow{2}{*}{$\begin{array}{c}\text { b3lyp/cc- } \\
\text { pvtz }\end{array}$} \\
\hline 0.779751 & -2.329428 & 0.000022 & & & & & \\
\hline 1.192364 & -2.845466 & -0.873791 & 1 & 0.113737 & 0.114034 & 0.104577 & 0.111216 \\
\hline-1.192308 & -2.845408 & -0.873898 & 1 & 0.104880 & 0.105605 & 0.098881 & 0.104615 \\
\hline-0.779753 & -2.329428 & -0.000022 & 12 & -0.108423 & -0.106340 & -0.094121 & -0.104156 \\
\hline 1.162886 & -0.876577 & 0.000000 & 12 & 0.219649 & 0.205255 & 0.213194 & 0.185924 \\
\hline 0.000000 & -0.090311 & 0.000000 & 12 & -0.044705 & -0.028613 & -0.066642 & 0.001218 \\
\hline 2.412113 & -0.228187 & -0.000009 & 12 & -0.213082 & -0.206236 & -0.191289 & -0.194840 \\
\hline 0.000000 & 1.320505 & 0.000000 & 12 & 0.112323 & 0.105194 & 0.076612 & 0.085017 \\
\hline-1.162886 & -0.876577 & -0.000001 & 12 & 0.209996 & 0.196560 & 0.203380 & 0.179922 \\
\hline 2.442165 & 1.167849 & -0.000007 & 12 & -0.033683 & -0.034128 & -0.025452 & -0.029735 \\
\hline 3.337654 & -0.791538 & -0.000010 & 1 & 0.185466 & 0.183958 & 0.174180 & 0.179414 \\
\hline 1.267047 & 1.947744 & 0.000016 & 12 & -0.119090 & -0.116026 & -0.083148 & -0.108621 \\
\hline-1.267046 & 1.947744 & -0.000016 & 12 & -0.121303 & -0.117494 & -0.086923 & -0.109020 \\
\hline-2.412113 & -0.228185 & 0.000009 & 12 & -0.208594 & -0.202675 & -0.186440 & -0.194242 \\
\hline 3.400094 & 1.674138 & -0.000015 & 1 & 0.166192 & 0.164764 & 0.154958 & 0.160592 \\
\hline 1.343234 & 3.029271 & 0.000004 & 1 & 0.174000 & 0.173026 & 0.160239 & 0.169936 \\
\hline-2.442165 & 1.167851 & 0.000007 & 12 & -0.031406 & -0.032257 & -0.021567 & -0.027540 \\
\hline-1.343231 & 3.029271 & -0.000004 & 1 & 0.175354 & 0.174012 & 0.161496 & 0.170159 \\
\hline-3.337654 & -0.791536 & 0.000010 & 1 & 0.182976 & 0.181826 & 0.172021 & 0.178387 \\
\hline-3.400094 & 1.674141 & 0.000015 & 1 & 0.164649 & 0.163487 & 0.153065 & 0.159578 \\
\hline-1.192366 & -2.845466 & 0.873791 & 1 & 0.104877 & 0.105603 & 0.098879 & 0.104613 \\
\hline 1.192306 & -2.845409 & 0.873898 & 1 & 0.113739 & 0.114036 & 0.104579 & 0.111218 \\
\hline
\end{tabular}




\begin{tabular}{|c|c|c|c|c|c|c|c|}
\hline \multicolumn{8}{|c|}{ Fluorene } \\
\hline \multicolumn{4}{|c|}{ Atom Orientation } & \multicolumn{4}{|c|}{ Electrostatic Potential Charges } \\
\hline \multicolumn{3}{|c|}{ Coordinates $(\mathrm{x}, \mathrm{y}, \mathrm{z})$} & \multirow{2}{*}{$\begin{array}{c}\text { Mass } \\
12 \\
\end{array}$} & \multirow{2}{*}{$\begin{array}{c}\text { b3lyp/6- } \\
31+G(d) \\
-0.145618 \\
\end{array}$} & \multirow{2}{*}{$\begin{array}{c}\text { b3lyp/6- } \\
31++G(d, p) \\
-0.142141 \\
\end{array}$} & \multirow{2}{*}{$\begin{array}{c}\begin{array}{c}\text { b3lyp/cc- } \\
\text { pvdz }\end{array} \\
-0.139703\end{array}$} & \multirow{2}{*}{$\begin{array}{c}\begin{array}{c}\text { b3lyp/cc- } \\
\text { pvtz }\end{array} \\
-0.134769\end{array}$} \\
\hline 0.000000 & -1.239142 & 2.969901 & & & & & \\
\hline-0.000004 & -1.523608 & 1.625460 & 12 & -0.109690 & -0.109274 & -0.083951 & -0.110155 \\
\hline-0.000004 & -0.441598 & 0.710639 & 12 & 0.047320 & 0.047715 & 0.034609 & 0.047555 \\
\hline 0.000000 & 0.916078 & 1.174656 & 12 & 0.262693 & 0.261689 & 0.235166 & 0.276992 \\
\hline 0.000005 & 1.183580 & 2.524295 & 12 & -0.277883 & -0.274216 & -0.253899 & -0.270024 \\
\hline 0.000004 & 0.102601 & 3.418225 & 12 & 0.078687 & 0.078173 & 0.086424 & 0.075449 \\
\hline 0.000000 & -2.037885 & 3.697108 & 1 & 0.170425 & 0.168498 & 0.162526 & 0.164859 \\
\hline-0.000008 & -2.545334 & 1.274301 & 1 & 0.163673 & 0.162736 & 0.153146 & 0.160210 \\
\hline 0.000008 & 2.197171 & 2.899473 & 1 & 0.191266 & 0.189946 & 0.180098 & 0.187079 \\
\hline 0.000008 & 0.297427 & 4.481970 & 1 & 0.141644 & 0.140468 & 0.133947 & 0.138868 \\
\hline 0.000000 & 0.916078 & 1.174656 & 12 & 0.262693 & 0.261689 & 0.235166 & 0.276992 \\
\hline-0.000004 & -0.441598 & 0.710639 & 12 & 0.047320 & 0.047715 & 0.034609 & 0.047556 \\
\hline 0.000005 & 1.183580 & 2.524295 & 12 & -0.277883 & -0.274216 & -0.253899 & -0.270024 \\
\hline-0.000004 & -1.523608 & 1.625460 & 12 & -0.109690 & -0.109274 & -0.083951 & -0.110155 \\
\hline 0.000004 & 0.102601 & 3.418225 & 12 & 0.078687 & 0.078173 & 0.086424 & 0.075448 \\
\hline 0.000008 & 2.197171 & 2.899473 & 1 & 0.191266 & 0.189946 & 0.180098 & 0.187079 \\
\hline 0.000000 & -1.239142 & 2.969901 & 12 & -0.145618 & -0.142141 & -0.139703 & -0.134769 \\
\hline-0.000008 & -2.545334 & 1.274301 & 1 & 0.163673 & 0.162736 & 0.153146 & 0.160210 \\
\hline 0.000008 & 0.297427 & 4.481970 & 1 & 0.141644 & 0.140468 & 0.133947 & 0.138868 \\
\hline 0.000000 & -2.037885 & 3.697108 & 1 & 0.170425 & 0.168498 & 0.162526 & 0.164859 \\
\hline-0.000002 & 1.862271 & 0.000000 & 12 & -0.396514 & -0.404155 & -0.343909 & -0.441661 \\
\hline-0.876752 & 2.514333 & 0.000000 & 1 & 0.175739 & 0.178482 & 0.163591 & 0.184766 \\
\hline 0.876739 & 2.514344 & 0.000000 & 1 & 0.175740 & 0.178483 & 0.163591 & 0.184766 \\
\hline
\end{tabular}




\begin{tabular}{|c|c|c|c|c|c|c|c|}
\hline \multicolumn{8}{|c|}{ Anthracene } \\
\hline \multicolumn{4}{|c|}{ Atom Orientation } & \multicolumn{4}{|c|}{ Electrostatic Potential Charges } \\
\hline \multicolumn{3}{|c|}{ Coordinates $(\mathrm{x}, \mathrm{y}, \mathrm{z})$} & \multirow{2}{*}{$\begin{array}{c}\text { Mass } \\
12\end{array}$} & \multirow{2}{*}{$\begin{array}{l}\text { b3lyp/6- } \\
31+G(d) \\
-0.06693\end{array}$} & \multirow{2}{*}{$\begin{array}{c}\text { b3lyp/6- } \\
31++\mathrm{G}(\mathrm{d}, \mathrm{p}) \\
-0.065708\end{array}$} & \multirow{2}{*}{$\begin{array}{c}\begin{array}{c}\text { b3lyp/cc- } \\
\text { pvdz }\end{array} \\
-0.055428\end{array}$} & \multirow{2}{*}{$\begin{array}{c}\text { b3lyp/cc- } \\
\text { pvtz }\end{array}$} \\
\hline 3.66536 & 0.70233 & 0.00000 & & & & & \\
\hline 2.46685 & 1.40631 & 0.00000 & 12 & -0.14632 & -0.144944 & -0.108469 & -0.145534 \\
\hline 1.23136 & 0.71957 & 0.00000 & 12 & 0.12693 & 0.128618 & 0.080493 & 0.133524 \\
\hline 1.23136 & -0.71957 & 0.00000 & 12 & 0.12693 & 0.128618 & 0.080493 & 0.133524 \\
\hline 2.46685 & -1.40631 & 0.00000 & 12 & -0.14632 & -0.144944 & -0.108469 & -0.145533 \\
\hline 3.66536 & -0.70233 & 0.00000 & 12 & -0.06693 & -0.065708 & -0.055428 & -0.059597 \\
\hline 0.00000 & 1.40441 & 0.00000 & 12 & -0.15936 & -0.163824 & -0.094952 & -0.172933 \\
\hline 0.00000 & -1.40441 & 0.00000 & 12 & -0.15936 & -0.163824 & -0.094952 & -0.172933 \\
\hline-1.23136 & -0.71957 & 0.00000 & 12 & 0.10928 & 0.112075 & 0.069045 & 0.120515 \\
\hline-1.23136 & 0.71957 & 0.00000 & 12 & 0.10928 & 0.112075 & 0.069045 & 0.120515 \\
\hline-2.46685 & 1.40631 & 0.00000 & 12 & -0.12761 & -0.126855 & -0.096911 & -0.129976 \\
\hline-2.47169 & 2.49046 & 0.00000 & 1 & 0.16602 & 0.164969 & 0.151944 & 0.163235 \\
\hline-3.66536 & 0.70233 & 0.00000 & 12 & -0.06959 & -0.068821 & -0.056655 & -0.063554 \\
\hline-3.66536 & -0.70233 & 0.00000 & 12 & -0.06959 & -0.068821 & -0.056655 & -0.063554 \\
\hline-2.46685 & -1.40631 & 0.00000 & 12 & -0.12761 & -0.126855 & -0.096911 & -0.129976 \\
\hline 0.00000 & 2.48991 & 0.00000 & 1 & 0.17012 & 0.170876 & 0.152536 & 0.169642 \\
\hline 4.60573 & 1.24001 & 0.00000 & 1 & 0.16429 & 0.162764 & 0.152480 & 0.158964 \\
\hline 2.47169 & 2.49046 & 0.00000 & 1 & 0.16994 & 0.168812 & 0.154317 & 0.166631 \\
\hline 2.47169 & -2.49046 & 0.00000 & 1 & 0.16994 & 0.168812 & 0.154317 & 0.166631 \\
\hline 4.60573 & -1.24001 & 0.00000 & 1 & 0.16429 & 0.162764 & 0.152480 & 0.158964 \\
\hline 0.00000 & -2.48991 & 0.00000 & 1 & 0.17012 & 0.170876 & 0.152536 & 0.169642 \\
\hline-4.60573 & 1.24001 & 0.00000 & 1 & 0.16322 & 0.162037 & 0.151600 & 0.159082 \\
\hline-4.60573 & -1.24001 & 0.00000 & 1 & 0.16322 & 0.162037 & 0.151600 & 0.159082 \\
\hline-2.47169 & -2.49046 & 0.00000 & 1 & 0.16602 & 0.164969 & 0.151944 & 0.163235 \\
\hline
\end{tabular}




\begin{tabular}{|c|c|c|c|c|c|c|c|}
\hline \multicolumn{8}{|c|}{ Phenanthrene } \\
\hline \multicolumn{4}{|c|}{ Atom Orientation } & \multicolumn{4}{|c|}{ Electrostatic Potential Charges } \\
\hline \multicolumn{3}{|c|}{ Coordinates $(\mathrm{x}, \mathrm{y}, \mathrm{z})$} & \multirow{2}{*}{$\begin{array}{c}\text { Mass } \\
12\end{array}$} & \multirow{2}{*}{$\begin{array}{c}\text { b3lyp/6- } \\
31+G(d) \\
-0.081823\end{array}$} & \multirow{2}{*}{$\begin{array}{c}\begin{array}{c}\text { b3lyp/6- } \\
31++G(d, p)\end{array} \\
-0.079176\end{array}$} & \multirow{2}{*}{$\begin{array}{c}\begin{array}{c}\text { b3lyp/cc- } \\
\text { pvdz }\end{array} \\
-0.085092\end{array}$} & \multirow{2}{*}{$\begin{array}{c}\text { b3lyp/cc- } \\
\text { pvtz }\end{array}$} \\
\hline 0.000000 & 3.578396 & -0.281698 & & & & & \\
\hline 0.000000 & 2.851750 & 0.887106 & 12 & -0.164108 & -0.163036 & -0.121101 & -0.150307 \\
\hline 0.000000 & 1.426625 & 0.858513 & 12 & 0.199966 & 0.200075 & 0.137459 & 0.178519 \\
\hline 0.000000 & 0.731566 & -0.395850 & 12 & 0.044502 & 0.043232 & 0.041819 & 0.050320 \\
\hline 0.000000 & 1.498665 & -1.558220 & 12 & -0.162340 & -0.160126 & -0.142742 & -0.162360 \\
\hline 0.000000 & 2.896860 & -1.504380 & 12 & 0.000510 & 0.001422 & 0.015277 & 0.008053 \\
\hline 0.000000 & 0.698749 & 2.056675 & 12 & -0.137346 & -0.136406 & -0.091850 & -0.122776 \\
\hline 0.000000 & -0.731566 & -0.395850 & 12 & 0.044502 & 0.043232 & 0.041819 & 0.050320 \\
\hline 0.000000 & -1.426625 & 0.858513 & 12 & 0.199966 & 0.200075 & 0.137459 & 0.178519 \\
\hline 0.000000 & -0.698749 & 2.056675 & 12 & -0.137346 & -0.136406 & -0.091850 & -0.122776 \\
\hline 0.000000 & -2.851750 & 0.887106 & 12 & -0.164108 & -0.163036 & -0.121101 & -0.150307 \\
\hline 0.000000 & -3.351002 & 1.846492 & 1 & 0.163157 & 0.161954 & 0.151928 & 0.158242 \\
\hline 0.000000 & -3.578396 & -0.281698 & 12 & -0.081823 & -0.079176 & -0.085092 & -0.080849 \\
\hline 0.000000 & -2.896860 & -1.504380 & 12 & 0.000510 & 0.001422 & 0.015277 & 0.008053 \\
\hline 0.000000 & -1.498665 & -1.558220 & 12 & -0.162340 & -0.160126 & -0.142742 & -0.162360 \\
\hline 0.000000 & 1.229996 & 2.999027 & 1 & 0.180338 & 0.179568 & 0.161651 & 0.173809 \\
\hline 0.000000 & 4.658048 & -0.259685 & 1 & 0.154044 & 0.152067 & 0.147753 & 0.150767 \\
\hline 0.000000 & 3.351002 & 1.846492 & 1 & 0.163157 & 0.161954 & 0.151928 & 0.158242 \\
\hline 0.000000 & 1.027502 & -2.529139 & 1 & 0.160366 & 0.159145 & 0.150792 & 0.157586 \\
\hline 0.000000 & 3.456344 & -2.429564 & 1 & 0.142734 & 0.141281 & 0.134106 & 0.138996 \\
\hline 0.000000 & -1.229996 & 2.999027 & 1 & 0.180338 & 0.179568 & 0.161651 & 0.173809 \\
\hline 0.000000 & -4.658048 & -0.259685 & 1 & 0.154044 & 0.152067 & 0.147753 & 0.150767 \\
\hline 0.000000 & -3.456344 & -2.429564 & 1 & 0.142734 & 0.141281 & 0.134106 & 0.138996 \\
\hline 0.000000 & -1.027502 & -2.529139 & 1 & 0.160366 & 0.159145 & 0.150792 & 0.157586 \\
\hline
\end{tabular}




\begin{tabular}{|c|c|c|c|c|c|c|c|}
\hline \multicolumn{8}{|c|}{ Fluoranthene } \\
\hline \multicolumn{4}{|c|}{ Atom Orientation } & \multicolumn{4}{|c|}{ Electrostatic Potential Charges } \\
\hline \multicolumn{3}{|c|}{ Coordinates $(\mathrm{x}, \mathrm{y}, \mathrm{z})$} & \multirow{2}{*}{$\begin{array}{c}\text { Mass } \\
12\end{array}$} & \multirow{2}{*}{$\begin{array}{c}\text { b31yp/6- } \\
31+G(d) \\
-0.147232\end{array}$} & \multirow{2}{*}{$\begin{array}{c}\begin{array}{c}\text { b3lyp/6- } \\
31++G(d, p)\end{array} \\
-0.145233\end{array}$} & \multirow{2}{*}{$\begin{array}{c}\begin{array}{c}\text { b3lyp/cc- } \\
\text { pvdz }\end{array} \\
-0.134480\end{array}$} & \multirow{2}{*}{$\begin{array}{c}\begin{array}{c}\text { b3lyp/cc- } \\
\text { pvtz }\end{array} \\
-0.136030\end{array}$} \\
\hline-4.360667 & -1.644471 & 0.000000 & & & & & \\
\hline-3.058663 & -2.091313 & 0.000000 & 12 & -0.076495 & -0.077721 & -0.052668 & -0.093146 \\
\hline-1.963847 & -1.174449 & 0.000000 & 12 & -0.020687 & -0.013327 & -0.028024 & 0.028587 \\
\hline-2.231907 & 0.248739 & 0.000000 & 12 & 0.227948 & 0.221301 & 0.176102 & 0.183901 \\
\hline-3.561786 & 0.662107 & 0.000000 & 12 & -0.216305 & -0.212001 & -0.175736 & -0.198064 \\
\hline-4.610443 & -0.263513 & 0.000000 & 12 & 0.005736 & 0.006467 & 0.014704 & 0.008458 \\
\hline-0.647054 & -1.677001 & 0.000000 & 12 & -0.017066 & -0.021498 & -0.018420 & -0.057359 \\
\hline-1.105231 & 1.179013 & 0.000000 & 12 & -0.121898 & -0.118130 & -0.081994 & -0.077429 \\
\hline 0.169825 & 0.613264 & 0.000000 & 12 & 0.258466 & 0.257755 & 0.155499 & 0.211899 \\
\hline 0.435083 & -0.785803 & 0.000000 & 12 & -0.125218 & -0.124319 & -0.033018 & -0.077615 \\
\hline 1.376956 & 1.345556 & 0.000000 & 12 & -0.181971 & -0.182485 & -0.110996 & -0.146354 \\
\hline 1.310157 & 2.724113 & 0.000000 & 12 & -0.060628 & -0.060613 & -0.065762 & -0.076828 \\
\hline 0.031042 & 3.325435 & 0.000001 & 12 & -0.105114 & -0.100439 & -0.082076 & -0.078074 \\
\hline-1.151943 & 2.587217 & 0.000000 & 12 & -0.113773 & -0.116051 & -0.101886 & -0.138235 \\
\hline-0.491854 & -2.750462 & 0.000000 & 1 & 0.146420 & 0.147102 & 0.139035 & 0.149475 \\
\hline-5.184298 & -2.346861 & 0.000000 & 1 & 0.166736 & 0.165186 & 0.155450 & 0.161743 \\
\hline-2.841822 & -3.153570 & 0.000000 & 1 & 0.150919 & 0.150144 & 0.138900 & 0.151029 \\
\hline-3.799909 & 1.718033 & 0.000000 & 1 & 0.169156 & 0.167369 & 0.153830 & 0.162024 \\
\hline-5.632474 & 0.097110 & 0.000000 & 1 & 0.151879 & 0.150453 & 0.140356 & 0.146904 \\
\hline 2.196816 & 3.346395 & 0.000000 & 1 & 0.155152 & 0.154285 & 0.145846 & 0.153310 \\
\hline-0.033371 & 4.407233 & 0.000001 & 1 & 0.158634 & 0.156403 & 0.145771 & 0.150498 \\
\hline-2.095971 & 3.116898 & 0.000001 & 1 & 0.153464 & 0.153112 & 0.142410 & 0.154792 \\
\hline 2.468575 & 0.342819 & 0.000000 & 12 & 0.136418 & 0.136416 & 0.101052 & 0.123043 \\
\hline 1.871985 & -0.957288 & 0.000000 & 12 & 0.141806 & 0.141715 & 0.066083 & 0.118963 \\
\hline 3.839693 & 0.468098 & 0.000000 & 12 & -0.162188 & -0.161174 & -0.133100 & -0.153355 \\
\hline 2.661942 & -2.110215 & 0.000001 & 12 & -0.123341 & -0.122792 & -0.081765 & -0.116152 \\
\hline 4.631565 & -0.706252 & 0.000000 & 12 & -0.023241 & -0.021493 & -0.013815 & -0.020236 \\
\hline 4.319691 & 1.439484 & 0.000000 & 1 & 0.165396 & 0.164332 & 0.152127 & 0.160002 \\
\hline 4.054249 & -1.971805 & 0.000000 & 12 & -0.154846 & -0.151544 & -0.140720 & -0.143906 \\
\hline 2.211419 & -3.096062 & 0.000001 & 1 & 0.159054 & 0.158053 & 0.143712 & 0.154796 \\
\hline 5.710959 & -0.611093 & 0.000000 & 1 & 0.142267 & 0.140451 & 0.133714 & 0.138615 \\
\hline 4.683632 & -2.852613 & 0.000000 & 1 & 0.160551 & 0.158278 & 0.149867 & 0.154743 \\
\hline
\end{tabular}




\begin{tabular}{|c|c|c|c|c|c|c|c|}
\hline \multicolumn{8}{|c|}{ Pyrene } \\
\hline \multicolumn{4}{|c|}{ Atom Orientation } & \multicolumn{4}{|c|}{ Electrostatic Potential Charges } \\
\hline \multicolumn{3}{|c|}{ Coordinates $(\mathrm{x}, \mathrm{y}, \mathrm{z})$} & \multirow{2}{*}{$\begin{array}{c}\text { Mass } \\
12\end{array}$} & \multirow{2}{*}{$\begin{array}{c}\text { b3lyp/6- } \\
31+G(d) \\
-0.121582\end{array}$} & \multirow{2}{*}{$\begin{array}{c}\begin{array}{c}\text { b3lyp/6- } \\
31++G(d, p)\end{array} \\
-0.156997\end{array}$} & \multirow{2}{*}{$\begin{array}{c}\begin{array}{c}\text { b3lyp/cc- } \\
\text { pvdz }\end{array} \\
-0.102269\end{array}$} & \multirow{2}{*}{$\begin{array}{c}\begin{array}{c}\text { b3lyp/cc- } \\
\text { pvtz }\end{array} \\
-0.150356\end{array}$} \\
\hline 0.000000 & -1.205595 & 2.841608 & & & & & \\
\hline 0.000000 & 1.232551 & 1.419803 & 12 & 0.148992 & 0.191130 & 0.135590 & 0.182494 \\
\hline 0.000000 & 1.205595 & 2.841608 & 12 & -0.121582 & -0.156997 & -0.102269 & -0.150356 \\
\hline 0.000000 & 0.000000 & 3.537325 & 12 & -0.082477 & -0.041125 & -0.065894 & -0.038941 \\
\hline 0.000000 & -2.144686 & 3.384033 & 1 & 0.163161 & 0.165857 & 0.152058 & 0.162770 \\
\hline 0.000000 & 2.144686 & 3.384033 & 1 & 0.163161 & 0.165857 & 0.152058 & 0.162770 \\
\hline 0.000000 & 0.000000 & 4.620015 & 1 & 0.158388 & 0.148408 & 0.146149 & 0.146600 \\
\hline 0.000000 & 0.000000 & 0.707815 & 12 & 0.001517 & -0.023463 & -0.012305 & -0.023569 \\
\hline 0.000000 & 0.000000 & -0.707815 & 12 & 0.001517 & -0.023463 & -0.012305 & -0.023569 \\
\hline 0.000000 & -1.232551 & 1.419803 & 12 & 0.148992 & 0.191130 & 0.135590 & 0.182494 \\
\hline 0.000000 & -1.232551 & -1.419803 & 12 & 0.148992 & 0.191130 & 0.135590 & 0.182494 \\
\hline 0.000000 & -2.448634 & 0.692064 & 12 & -0.153958 & -0.171310 & -0.132948 & -0.161210 \\
\hline 0.000000 & -2.448634 & -0.692064 & 12 & -0.153958 & -0.171310 & -0.132948 & -0.161210 \\
\hline 0.000000 & -3.388518 & 1.232375 & 1 & 0.174674 & 0.179410 & 0.163595 & 0.174258 \\
\hline 0.000000 & -3.388518 & -1.232375 & 1 & 0.174674 & 0.179410 & 0.163595 & 0.174258 \\
\hline 0.000000 & 2.448634 & 0.692064 & 12 & -0.153958 & -0.171310 & -0.132948 & -0.161210 \\
\hline 0.000000 & 2.448634 & -0.692064 & 12 & -0.153958 & -0.171310 & -0.132948 & -0.161210 \\
\hline 0.000000 & 3.388518 & 1.232375 & 1 & 0.174674 & 0.179410 & 0.163595 & 0.174258 \\
\hline 0.000000 & 3.388518 & -1.232375 & 1 & 0.174674 & 0.179410 & 0.163595 & 0.174258 \\
\hline 0.000000 & 1.232551 & -1.419803 & 12 & 0.148992 & 0.191130 & 0.135590 & 0.182494 \\
\hline 0.000000 & 1.205595 & -2.841608 & 12 & -0.121582 & -0.156997 & -0.102269 & -0.150356 \\
\hline 0.000000 & 0.000000 & -3.537325 & 12 & -0.082477 & -0.041125 & -0.065894 & -0.038941 \\
\hline 0.000000 & 2.144686 & -3.384033 & 1 & 0.163161 & 0.165857 & 0.152058 & 0.162770 \\
\hline 0.000000 & -1.205595 & -2.841608 & 12 & -0.121582 & -0.156997 & -0.102269 & -0.150356 \\
\hline 0.000000 & 0.000000 & -4.620015 & 1 & 0.158388 & 0.148408 & 0.146149 & 0.146600 \\
\hline 0.000000 & -2.144686 & -3.384033 & 1 & 0.163161 & 0.165857 & 0.152058 & 0.162770 \\
\hline
\end{tabular}




\begin{tabular}{|c|c|c|c|c|c|c|c|}
\hline \multicolumn{8}{|c|}{ 1,2-Benzanthracene } \\
\hline \multicolumn{4}{|c|}{ Atom Orientation } & \multicolumn{4}{|c|}{ Electrostatic Potential Charges } \\
\hline \multicolumn{3}{|c|}{ Coordinates $(\mathrm{x}, \mathrm{y}, \mathrm{z})$} & Mass & $\begin{array}{l}\text { b3lyp/6- } \\
31+G(d)\end{array}$ & $\begin{array}{c}\text { b3lyp/6- } \\
31++\mathrm{G}(\mathrm{d}, \mathrm{p})\end{array}$ & $\begin{array}{l}\text { b3lyp/cc- } \\
\text { pvdz }\end{array}$ & $\begin{array}{c}\text { b3lyp/cc- } \\
\text { pvtz }\end{array}$ \\
\hline 4.765370 & -0.015140 & 0.000000 & 12 & -0.094566 & -0.092515 & -0.080066 & -0.084206 \\
\hline 3.805658 & 0.988527 & 0.000000 & 12 & -0.148681 & -0.147278 & -0.108969 & -0.147528 \\
\hline 2.428530 & 0.662611 & 0.000000 & 12 & 0.137456 & 0.139372 & 0.085272 & 0.143313 \\
\hline 2.031904 & -0.714595 & 0.000000 & 12 & 0.095030 & 0.096516 & 0.058303 & 0.106571 \\
\hline 3.027834 & -1.711451 & 0.000000 & 12 & -0.153390 & -0.151525 & -0.116402 & -0.152477 \\
\hline 4.376277 & -1.363814 & 0.000000 & 12 & -0.049549 & -0.050190 & -0.041939 & -0.049741 \\
\hline 1.422666 & 1.645534 & 0.000000 & 12 & -0.160015 & -0.164950 & -0.099602 & -0.175807 \\
\hline 0.651224 & -1.037368 & 0.000000 & 12 & -0.117113 & -0.118337 & -0.069931 & -0.128848 \\
\hline-0.351511 & -0.065447 & 0.000000 & 12 & -0.016911 & -0.019222 & -0.024024 & -0.020308 \\
\hline 0.052493 & 1.314450 & 0.000000 & 12 & 0.127263 & 0.132380 & 0.090553 & 0.145033 \\
\hline-0.927144 & 2.339731 & 0.000000 & 12 & -0.160342 & -0.160261 & -0.130942 & -0.163687 \\
\hline-0.607283 & 3.375382 & 0.000000 & 1 & 0.173022 & 0.172135 & 0.156871 & 0.170177 \\
\hline-2.270385 & 2.029601 & 0.000000 & 12 & -0.122644 & -0.122160 & -0.084451 & -0.113737 \\
\hline-2.722935 & 0.686609 & 0.000000 & 12 & 0.135530 & 0.134775 & 0.098367 & 0.125312 \\
\hline-1.768228 & -0.390487 & 0.000000 & 12 & 0.142297 & 0.142674 & 0.114235 & 0.151720 \\
\hline 1.705780 & 2.693355 & 0.000000 & 1 & 0.161491 & 0.162214 & 0.145741 & 0.161395 \\
\hline 5.817378 & 0.242471 & 0.000000 & 1 & 0.164625 & 0.162995 & 0.151836 & 0.158900 \\
\hline 4.104224 & 2.030852 & 0.000000 & 1 & 0.168719 & 0.167455 & 0.152150 & 0.164874 \\
\hline 2.737249 & -2.756024 & 0.000000 & 1 & 0.164564 & 0.163365 & 0.148700 & 0.161608 \\
\hline 5.130906 & -2.141089 & 0.000000 & 1 & 0.156080 & 0.155106 & 0.145357 & 0.153093 \\
\hline 0.389134 & -2.088096 & 0.000000 & 1 & 0.150384 & 0.150368 & 0.137807 & 0.149603 \\
\hline-3.006626 & 2.825741 & 0.000000 & 1 & 0.168667 & 0.167945 & 0.151936 & 0.164153 \\
\hline-4.113174 & 0.400170 & 0.000000 & 12 & -0.195237 & -0.193043 & -0.158887 & -0.187650 \\
\hline-4.559348 & -0.900204 & 0.000000 & 12 & -0.094786 & -0.092302 & -0.083122 & -0.083420 \\
\hline-4.815340 & 1.225847 & 0.000000 & 1 & 0.167716 & 0.166101 & 0.153010 & 0.162002 \\
\hline-2.263910 & -1.709583 & 0.000000 & 12 & -0.249212 & -0.245737 & -0.211804 & -0.247268 \\
\hline-3.624358 & -1.959153 & 0.000000 & 12 & -0.028747 & -0.029669 & -0.018987 & -0.028428 \\
\hline-5.620318 & -1.117516 & 0.000000 & 1 & 0.160736 & 0.158894 & 0.148595 & 0.154365 \\
\hline-1.585533 & -2.551951 & 0.000000 & 1 & 0.169449 & 0.167646 & 0.153626 & 0.166093 \\
\hline-3.978097 & -2.983414 & 0.000000 & 1 & 0.148165 & 0.147247 & 0.136766 & 0.144889 \\
\hline
\end{tabular}




\begin{tabular}{|c|c|c|c|c|c|c|c|}
\hline \multicolumn{8}{|c|}{ 2,3-Benzanthracene } \\
\hline \multicolumn{4}{|c|}{ Atom Orientation } & \multicolumn{4}{|c|}{ Electrostatic Potential Charges } \\
\hline \multicolumn{3}{|c|}{ Coordinates $(\mathrm{x}, \mathrm{y}, \mathrm{z})$} & Mass & $\begin{array}{l}\text { b3lyp/6- } \\
31+G(d)\end{array}$ & $\begin{array}{c}\text { b3lyp/6- } \\
31++\mathrm{G}(\mathrm{d}, \mathrm{p})\end{array}$ & $\begin{array}{l}\text { b3lyp/cc- } \\
\text { pvdz }\end{array}$ & $\begin{array}{c}\text { b3lyp/cc- } \\
\text { pvtz }\end{array}$ \\
\hline 0.000000 & 4.889490 & 0.707115 & 12 & -0.068502 & -0.067013 & -0.056210 & -0.061539 \\
\hline 0.000000 & 3.699122 & 1.409913 & 12 & -0.175644 & -0.174559 & -0.146619 & -0.175427 \\
\hline 0.000000 & 2.458000 & 0.722471 & 12 & 0.174601 & 0.175036 & 0.127140 & 0.174325 \\
\hline 0.000000 & 2.458000 & -0.722471 & 12 & 0.174601 & 0.175036 & 0.127140 & 0.174325 \\
\hline 0.000000 & 3.699122 & -1.409913 & 12 & -0.175644 & -0.174559 & -0.146619 & -0.175427 \\
\hline 0.000000 & 4.889490 & -0.707115 & 12 & -0.068502 & -0.067013 & -0.056210 & -0.061539 \\
\hline 0.000000 & 1.229196 & 1.408423 & 12 & -0.261375 & -0.263720 & -0.182204 & -0.259679 \\
\hline 0.000000 & 1.229196 & -1.408423 & 12 & -0.261375 & -0.263720 & -0.182204 & -0.259679 \\
\hline 0.000000 & 0.000000 & -0.725437 & 12 & 0.161539 & 0.164021 & 0.104041 & 0.163783 \\
\hline 0.000000 & 0.000000 & 0.725437 & 12 & 0.161539 & 0.164021 & 0.104041 & 0.163783 \\
\hline 0.000000 & -1.229196 & 1.408423 & 12 & -0.261375 & -0.263720 & -0.182204 & -0.259679 \\
\hline 0.000000 & -1.230955 & 2.495714 & 1 & 0.187552 & 0.188073 & 0.164515 & 0.184032 \\
\hline 0.000000 & -2.458000 & 0.722471 & 12 & 0.174601 & 0.175036 & 0.127140 & 0.174325 \\
\hline 0.000000 & -2.458000 & -0.722471 & 12 & 0.174601 & 0.175036 & 0.127140 & 0.174325 \\
\hline 0.000000 & -1.229196 & -1.408423 & 12 & -0.261375 & -0.263720 & -0.182204 & -0.259679 \\
\hline 0.000000 & 1.230955 & 2.495714 & 1 & 0.187552 & 0.188073 & 0.164515 & 0.184032 \\
\hline 0.000000 & 5.834163 & 1.241768 & 1 & 0.152811 & 0.151384 & 0.142774 & 0.148783 \\
\hline 0.000000 & 3.701751 & 2.496164 & 1 & 0.159788 & 0.158788 & 0.148583 & 0.157613 \\
\hline 0.000000 & 3.701751 & -2.496164 & 1 & 0.159788 & 0.158788 & 0.148583 & 0.157613 \\
\hline 0.000000 & 5.834163 & -1.241768 & 1 & 0.152811 & 0.151384 & 0.142774 & 0.148783 \\
\hline 0.000000 & 1.230955 & -2.495714 & 1 & 0.187552 & 0.188073 & 0.164515 & 0.184032 \\
\hline 0.000000 & -1.230955 & -2.495714 & 1 & 0.187552 & 0.188073 & 0.164515 & 0.184032 \\
\hline 0.000000 & -3.699122 & 1.409913 & 12 & -0.175644 & -0.174559 & -0.146619 & -0.175427 \\
\hline 0.000000 & -4.889490 & 0.707115 & 12 & -0.068502 & -0.067013 & -0.056210 & -0.061539 \\
\hline 0.000000 & -3.701751 & 2.496164 & 1 & 0.159788 & 0.158788 & 0.148583 & 0.157613 \\
\hline 0.000000 & -3.699122 & -1.409913 & 12 & -0.175644 & -0.174559 & -0.146619 & -0.175427 \\
\hline 0.000000 & -4.889490 & -0.707115 & 12 & -0.068502 & -0.067013 & -0.056210 & -0.061539 \\
\hline 0.000000 & -5.834163 & 1.241768 & 1 & 0.152811 & 0.151384 & 0.142774 & 0.148783 \\
\hline 0.000000 & -3.701751 & -2.496164 & 1 & 0.159788 & 0.158788 & 0.148583 & 0.157613 \\
\hline 0.000000 & -5.834163 & -1.241768 & 1 & 0.152811 & 0.151384 & 0.142774 & 0.148783 \\
\hline
\end{tabular}




\begin{tabular}{|c|c|c|c|c|c|c|c|}
\hline \multicolumn{8}{|c|}{ Chrysene } \\
\hline \multicolumn{4}{|c|}{ Atom Orientation } & \multicolumn{4}{|c|}{ Electrostatic Potential Charges } \\
\hline \multicolumn{3}{|c|}{ Coordinates $(\mathrm{x}, \mathrm{y}, \mathrm{z})$} & \multirow{2}{*}{$\begin{array}{c}\text { Mass } \\
12\end{array}$} & \multirow{2}{*}{$\begin{array}{c}\text { b3lyp/6- } \\
31+G(d) \\
-0.120373\end{array}$} & \multirow{2}{*}{$\begin{array}{c}\begin{array}{c}\text { b3lyp/6- } \\
31++G(d, p)\end{array} \\
-0.120777 \\
\end{array}$} & \multirow{2}{*}{$\begin{array}{c}\begin{array}{c}\text { b3lyp/cc- } \\
\text { pvdz }\end{array} \\
-0.091304\end{array}$} & \multirow{2}{*}{$\begin{array}{c}\begin{array}{c}\text { b3lyp/cc- } \\
\text { pvtz }\end{array} \\
-0.122678\end{array}$} \\
\hline-0.661378 & 3.923861 & 0.000000 & & & & & \\
\hline 1.794618 & 2.594647 & 0.000000 & 12 & -0.145431 & -0.144431 & -0.114793 & -0.151844 \\
\hline 1.741054 & 3.989607 & 0.000000 & 12 & -0.068822 & -0.066515 & -0.055860 & -0.057257 \\
\hline 0.513905 & 4.660058 & 0.000000 & 12 & -0.108115 & -0.105680 & -0.093536 & -0.097901 \\
\hline-1.622945 & 4.424745 & 0.000000 & 1 & 0.156495 & 0.155677 & 0.143754 & 0.153597 \\
\hline 2.768476 & 2.124518 & 0.000000 & 1 & 0.153339 & 0.152088 & 0.141042 & 0.151148 \\
\hline 2.665594 & 4.554457 & 0.000000 & 1 & 0.158817 & 0.156957 & 0.146164 & 0.152373 \\
\hline 0.482340 & 5.742433 & 0.000000 & 1 & 0.165296 & 0.163418 & 0.152503 & 0.158873 \\
\hline-0.626314 & 2.510150 & 0.000000 & 12 & 0.081244 & 0.083190 & 0.046787 & 0.083509 \\
\hline-1.826515 & 1.758410 & 0.000000 & 12 & -0.062349 & -0.062811 & -0.029900 & -0.061200 \\
\hline 0.622297 & 1.821058 & 0.000000 & 12 & 0.061559 & 0.060614 & 0.036381 & 0.067759 \\
\hline-1.823185 & 0.372771 & 0.000000 & 12 & -0.191163 & -0.189649 & -0.164731 & -0.187374 \\
\hline-2.773942 & 2.286613 & 0.000000 & 1 & 0.157533 & 0.157058 & 0.143048 & 0.154416 \\
\hline 0.626314 & 0.361792 & 0.000000 & 12 & 0.077103 & 0.076888 & 0.071920 & 0.075171 \\
\hline-0.626314 & -0.361792 & 0.000000 & 12 & 0.103725 & 0.103262 & 0.092495 & 0.099992 \\
\hline-2.775492 & -0.138249 & 0.000000 & 1 & 0.169963 & 0.169103 & 0.156640 & 0.167067 \\
\hline-0.622297 & -1.821058 & 0.000000 & 12 & 0.063512 & 0.062595 & 0.039062 & 0.070434 \\
\hline 0.626314 & -2.510150 & 0.000000 & 12 & 0.085380 & 0.086046 & 0.048417 & 0.081470 \\
\hline 1.823185 & -0.372771 & 0.000000 & 12 & -0.175730 & -0.173497 & -0.151454 & -0.170566 \\
\hline 1.826515 & -1.758410 & 0.000000 & 12 & -0.071368 & -0.071609 & -0.036868 & -0.067648 \\
\hline 2.775492 & 0.138249 & 0.000000 & 1 & 0.167638 & 0.166251 & 0.154351 & 0.163229 \\
\hline 2.773942 & -2.286613 & 0.000000 & 1 & 0.159934 & 0.159475 & 0.144827 & 0.156379 \\
\hline-1.794618 & -2.594647 & 0.000000 & 12 & -0.167742 & -0.165352 & -0.132664 & -0.167919 \\
\hline-1.741054 & -3.989607 & 0.000000 & 12 & -0.046507 & -0.046271 & -0.039479 & -0.044288 \\
\hline-2.768476 & -2.124518 & 0.000000 & 1 & 0.158213 & 0.156789 & 0.145261 & 0.155016 \\
\hline 0.661378 & -3.923861 & 0.000000 & 12 & -0.129534 & -0.127974 & -0.097242 & -0.124215 \\
\hline-0.513905 & -4.660058 & 0.000000 & 12 & -0.108498 & -0.106462 & -0.093478 & -0.098960 \\
\hline-2.665594 & -4.554457 & 0.000000 & 1 & 0.152478 & 0.151251 & 0.141543 & 0.148945 \\
\hline 1.622945 & -4.424745 & 0.000000 & 1 & 0.159094 & 0.157709 & 0.145490 & 0.153885 \\
\hline-0.482340 & -5.742433 & 0.000000 & 1 & 0.164310 & 0.162657 & 0.151623 & 0.158589 \\
\hline
\end{tabular}




\begin{tabular}{|c|c|c|c|c|c|c|c|}
\hline \multicolumn{8}{|c|}{ Triphenylene } \\
\hline \multicolumn{4}{|c|}{ Atom Orientation } & \multicolumn{4}{|c|}{ Electrostatic Potential Charges } \\
\hline \multicolumn{3}{|c|}{ Coordinates $(\mathrm{x}, \mathrm{y}, \mathrm{z})$} & Mass & $\begin{array}{l}\text { b3lyp/6- } \\
31+G(d)\end{array}$ & $\begin{array}{l}\text { b3lyp/6- } \\
31++\mathrm{G}(\mathrm{d}, \mathrm{p})\end{array}$ & $\begin{array}{l}\text { b3lyp/cc- } \\
\text { pvdz }\end{array}$ & $\begin{array}{l}\text { b3lyp/cc- } \\
\text { pvtz }\end{array}$ \\
\hline-1.273092 & -0.670520 & 0.000014 & 12 & 0.068220 & 0.069755 & 0.057524 & 0.076491 \\
\hline-0.048365 & -1.439474 & 0.000032 & 12 & 0.142054 & 0.135152 & 0.120133 & 0.133372 \\
\hline 1.241470 & -0.753819 & 0.000004 & 12 & -0.000679 & 0.004151 & -0.009315 & 0.006606 \\
\hline 1.291718 & 0.663677 & -0.000019 & 12 & 0.009009 & 0.008377 & -0.007169 & 0.008974 \\
\hline 0.056572 & 1.438144 & -0.000020 & 12 & 0.141833 & 0.135586 & 0.121611 & 0.134414 \\
\hline-1.222589 & 0.757302 & -0.000015 & 12 & 0.097113 & 0.100632 & 0.084549 & 0.100070 \\
\hline-2.417524 & 1.545790 & -0.000032 & 12 & -0.177275 & -0.177139 & -0.151064 & -0.175202 \\
\hline-2.372677 & 2.914077 & -0.000046 & 12 & -0.099379 & -0.098798 & -0.089283 & -0.098597 \\
\hline-3.382223 & 1.062257 & -0.000035 & 1 & 0.165651 & 0.165031 & 0.154553 & 0.162435 \\
\hline 0.058152 & 2.842559 & -0.000029 & 12 & -0.237513 & -0.230327 & -0.204344 & -0.229952 \\
\hline-1.120120 & 3.570264 & -0.000041 & 12 & 0.013298 & 0.013739 & 0.021669 & 0.022774 \\
\hline-3.288067 & 3.492314 & -0.000059 & 1 & 0.161581 & 0.160558 & 0.150710 & 0.158457 \\
\hline 0.990557 & 3.387234 & -0.000025 & 1 & 0.171311 & 0.167835 & 0.157530 & 0.165267 \\
\hline-1.079591 & 4.653185 & -0.000048 & 1 & 0.149084 & 0.147798 & 0.138263 & 0.143576 \\
\hline 2.552439 & 1.294007 & -0.000045 & 12 & -0.101222 & -0.100288 & -0.074466 & -0.101807 \\
\hline 2.452387 & -1.470312 & -0.000005 & 12 & -0.105540 & -0.107554 & -0.081311 & -0.107986 \\
\hline 3.740175 & 0.563577 & -0.000050 & 12 & -0.098629 & -0.096662 & -0.085805 & -0.089398 \\
\hline 2.622907 & 2.372191 & -0.000065 & 1 & 0.149635 & 0.149556 & 0.138645 & 0.147651 \\
\hline 3.690073 & -0.824944 & -0.000031 & 12 & -0.082485 & -0.080101 & -0.071402 & -0.074884 \\
\hline 2.446986 & -2.550812 & 0.000005 & 1 & 0.150487 & 0.151188 & 0.139794 & 0.148282 \\
\hline 4.690690 & 1.082393 & -0.000071 & 1 & 0.161264 & 0.159367 & 0.148999 & 0.155056 \\
\hline 4.600948 & -1.410714 & -0.000037 & 1 & 0.158644 & 0.156841 & 0.147073 & 0.153067 \\
\hline-2.522054 & -1.370968 & 0.000033 & 12 & -0.149444 & -0.148925 & -0.125835 & -0.156175 \\
\hline-0.146657 & -2.838394 & 0.000078 & 12 & -0.225817 & -0.219317 & -0.194326 & -0.222144 \\
\hline-2.576697 & -2.739865 & 0.000073 & 12 & -0.120578 & -0.118745 & -0.107216 & -0.111851 \\
\hline-3.449012 & -0.818631 & 0.000018 & 1 & 0.161986 & 0.160539 & 0.150597 & 0.160191 \\
\hline-1.375733 & -3.482011 & 0.000097 & 12 & 0.015315 & 0.016033 & 0.023272 & 0.024590 \\
\hline 0.744457 & -3.448454 & 0.000101 & 1 & 0.167545 & 0.164006 & 0.154446 & 0.163176 \\
\hline-3.531447 & -3.250377 & 0.000087 & 1 & 0.164754 & 0.163381 & 0.153263 & 0.159853 \\
\hline-1.411070 & -4.565130 & 0.000132 & 1 & 0.149776 & 0.148331 & 0.138905 & 0.143695 \\
\hline
\end{tabular}




\begin{tabular}{|c|c|c|c|c|c|c|c|}
\hline \multicolumn{8}{|c|}{ Benzo(a)pyrene } \\
\hline \multicolumn{4}{|c|}{ Atom Orientation } & \multicolumn{4}{|c|}{ Electrostatic Potential Charges } \\
\hline \multicolumn{3}{|c|}{ Coordinates $(\mathrm{x}, \mathrm{y}, \mathrm{z})$} & Mass & $\begin{array}{l}\text { b3lyp/6- } \\
31+G(d)\end{array}$ & $\begin{array}{c}\text { b3lyp/6- } \\
31++G(d, p)\end{array}$ & $\begin{array}{l}\text { b3lyp/cc- } \\
\text { pvdz }\end{array}$ & $\begin{array}{l}\text { b3lyp/cc- } \\
\text { pvtz }\end{array}$ \\
\hline 4.796228 & 0.159178 & 0.000000 & 12 & -0.124474 & -0.115664 & -0.105783 & -0.110029 \\
\hline 3.845803 & 1.152244 & -0.000001 & 12 & -0.146557 & -0.153634 & -0.103013 & -0.145555 \\
\hline 2.457279 & 0.842092 & -0.000001 & 12 & 0.235519 & 0.191258 & 0.061822 & 0.137478 \\
\hline 2.042089 & -0.531413 & 0.000000 & 12 & -0.019517 & -0.008961 & 0.052175 & 0.057250 \\
\hline 3.054702 & -1.527802 & 0.000003 & 12 & -0.173819 & -0.171379 & -0.143668 & -0.170686 \\
\hline 4.392496 & -1.196161 & 0.000003 & 12 & -0.031961 & -0.031443 & -0.032807 & -0.037905 \\
\hline 1.486002 & 1.870081 & -0.000002 & 12 & -0.314309 & -0.254521 & -0.106370 & -0.192645 \\
\hline 0.631761 & -0.835902 & -0.000001 & 12 & 0.151634 & 0.153083 & 0.077919 & 0.090158 \\
\hline-0.313418 & 0.223213 & 0.000000 & 12 & -0.107962 & -0.088734 & -0.050787 & -0.071998 \\
\hline 0.129930 & 1.595607 & -0.000001 & 12 & 0.249729 & 0.225905 & 0.123312 & 0.185778 \\
\hline-0.858126 & 2.646164 & 0.000000 & 12 & -0.161176 & -0.166286 & -0.150314 & -0.181156 \\
\hline-0.507538 & 3.675483 & 0.000000 & 1 & 0.167909 & 0.171534 & 0.159049 & 0.171391 \\
\hline-2.186442 & 2.370175 & 0.000000 & 12 & -0.178704 & -0.195879 & -0.086075 & -0.114602 \\
\hline-2.668383 & 1.011161 & 0.000000 & 12 & 0.144324 & 0.187016 & 0.054615 & 0.086813 \\
\hline-1.716004 & -0.056261 & 0.000000 & 12 & 0.078681 & 0.036392 & 0.075574 & 0.088852 \\
\hline 1.821662 & 2.905141 & -0.000002 & 1 & 0.192514 & 0.174953 & 0.145515 & 0.162460 \\
\hline 5.853349 & 0.410309 & 0.000000 & 1 & 0.162298 & 0.160397 & 0.152057 & 0.159237 \\
\hline 4.140936 & 2.198944 & -0.000002 & 1 & 0.153017 & 0.162252 & 0.146182 & 0.159444 \\
\hline 2.780428 & -2.576971 & 0.000005 & 1 & 0.155963 & 0.155197 & 0.143102 & 0.151837 \\
\hline 5.142528 & -1.982390 & 0.000005 & 1 & 0.148769 & 0.146749 & 0.141064 & 0.147854 \\
\hline-2.917696 & 3.174940 & 0.000000 & 1 & 0.178316 & 0.181767 & 0.149844 & 0.161643 \\
\hline 0.133166 & -2.178530 & -0.000002 & 12 & -0.148402 & -0.166400 & -0.139450 & -0.161902 \\
\hline-2.177727 & -1.405779 & -0.000001 & 12 & 0.123826 & 0.161644 & 0.022356 & 0.062525 \\
\hline-1.204925 & -2.451790 & -0.000002 & 12 & -0.190002 & -0.179001 & -0.085041 & -0.111723 \\
\hline 0.831731 & -3.007579 & -0.000004 & 1 & 0.162113 & 0.166422 & 0.150863 & 0.161236 \\
\hline-1.550244 & -3.482930 & -0.000004 & 1 & 0.181592 & 0.175453 & 0.148163 & 0.158462 \\
\hline-3.563988 & -1.662845 & 0.000000 & 12 & -0.123536 & -0.163397 & -0.045502 & -0.092865 \\
\hline-4.477369 & -0.618056 & 0.000000 & 12 & -0.081955 & -0.053199 & -0.123917 & -0.107087 \\
\hline-3.909184 & -2.693882 & 0.000000 & 1 & 0.155264 & 0.162590 & 0.141003 & 0.153730 \\
\hline-4.034264 & 0.708972 & 0.000002 & 12 & -0.155862 & -0.176692 & -0.075488 & -0.116505 \\
\hline-5.543184 & -0.829734 & 0.000002 & 1 & 0.153152 & 0.148992 & 0.156662 & 0.160453 \\
\hline-4.755645 & 1.522578 & 0.000002 & 1 & 0.163615 & 0.163589 & 0.146936 & 0.158057 \\
\hline
\end{tabular}




\begin{tabular}{|c|c|c|c|c|c|c|c|}
\hline \multicolumn{8}{|c|}{ Benzo(e)pyrene } \\
\hline \multicolumn{4}{|c|}{ Atom Orientation } & \multicolumn{4}{|c|}{ Electrostatic Potential Charges } \\
\hline \multicolumn{3}{|c|}{ Coordinates $(\mathrm{x}, \mathrm{y}, \mathrm{z})$} & \multirow{2}{*}{$\begin{array}{c}\text { Mass } \\
12\end{array}$} & \multirow{2}{*}{$\begin{array}{c}\text { b31yp/6- } \\
31+G(d) \\
-0.154646\end{array}$} & \multirow{2}{*}{$\begin{array}{c}\begin{array}{c}\text { b3lyp/6- } \\
31++G(d, p)\end{array} \\
-0.149487\end{array}$} & \multirow{2}{*}{$\begin{array}{c}\begin{array}{c}\text { b3lyp/cc- } \\
\text { pvdz }\end{array} \\
-0.143835\end{array}$} & \multirow{2}{*}{$\begin{array}{c}\begin{array}{c}\text { b3lyp/cc- } \\
\text { pvtz }\end{array} \\
-0.134139\end{array}$} \\
\hline 0.918616 & 3.541029 & 0.000000 & & & & & \\
\hline 2.110852 & 2.836303 & 0.000000 & 12 & -0.089873 & -0.091825 & -0.057209 & -0.093323 \\
\hline 2.105896 & 1.415616 & 0.000000 & 12 & 0.100795 & 0.104958 & 0.071258 & 0.107947 \\
\hline 0.863876 & 0.713162 & 0.000000 & 12 & 0.027303 & 0.024055 & 0.016884 & 0.017701 \\
\hline-0.357442 & 1.435676 & 0.000000 & 12 & -0.007390 & -0.003971 & -0.002173 & 0.006681 \\
\hline-0.293555 & 2.853643 & 0.000000 & 12 & -0.031797 & -0.034744 & -0.015549 & -0.048091 \\
\hline 3.320897 & 0.690883 & 0.000000 & 12 & -0.129702 & -0.130033 & -0.099693 & -0.126368 \\
\hline 0.863876 & -0.713162 & 0.000000 & 12 & 0.027302 & 0.024055 & 0.016884 & 0.017701 \\
\hline 2.105896 & -1.415616 & 0.000000 & 12 & 0.100795 & 0.104958 & 0.071258 & 0.107947 \\
\hline 3.320897 & -0.690883 & 0.000000 & 12 & -0.129702 & -0.130033 & -0.099693 & -0.126368 \\
\hline 2.110852 & -2.836303 & 0.000000 & 12 & -0.089873 & -0.091826 & -0.057209 & -0.093323 \\
\hline 3.060287 & -3.359621 & 0.000000 & 1 & 0.159779 & 0.159054 & 0.146078 & 0.155828 \\
\hline 0.918616 & -3.541029 & 0.000000 & 12 & -0.154646 & -0.149487 & -0.143835 & -0.134139 \\
\hline-0.293555 & -2.853643 & 0.000000 & 12 & -0.031797 & -0.034744 & -0.015549 & -0.048091 \\
\hline-0.357442 & -1.435676 & 0.000000 & 12 & -0.007390 & -0.003971 & -0.002172 & 0.006681 \\
\hline 4.259086 & 1.233935 & 0.000000 & 1 & 0.166986 & 0.166368 & 0.151994 & 0.162772 \\
\hline 0.922347 & 4.623677 & 0.000000 & 1 & 0.171347 & 0.169326 & 0.159399 & 0.164444 \\
\hline 3.060287 & 3.359621 & 0.000000 & 1 & 0.159779 & 0.159054 & 0.146078 & 0.155828 \\
\hline-1.205294 & 3.433856 & 0.000000 & 1 & 0.140781 & 0.140459 & 0.132193 & 0.141437 \\
\hline 4.259086 & -1.233935 & 0.000000 & 1 & 0.166986 & 0.166368 & 0.151994 & 0.162772 \\
\hline 0.922347 & -4.623677 & 0.000000 & 1 & 0.171347 & 0.169326 & 0.159399 & 0.164444 \\
\hline-1.205294 & -3.433856 & 0.000000 & 1 & 0.140781 & 0.140459 & 0.132193 & 0.141437 \\
\hline-1.616263 & -0.720153 & 0.000000 & 12 & 0.105849 & 0.104168 & 0.083335 & 0.104144 \\
\hline-1.616263 & 0.720153 & 0.000000 & 12 & 0.105849 & 0.104168 & 0.083336 & 0.104144 \\
\hline-2.860371 & 1.395653 & 0.000000 & 12 & -0.211586 & -0.207700 & -0.181450 & -0.206729 \\
\hline-4.051445 & 0.704932 & 0.000000 & 12 & -0.064806 & -0.064380 & -0.053187 & -0.060168 \\
\hline-2.892828 & 2.475424 & 0.000000 & 1 & 0.163785 & 0.161773 & 0.150439 & 0.158647 \\
\hline-2.860371 & -1.395653 & 0.000000 & 12 & -0.211586 & -0.207699 & -0.181450 & -0.206729 \\
\hline-4.051445 & -0.704933 & 0.000000 & 12 & -0.064806 & -0.064381 & -0.053187 & -0.060168 \\
\hline-4.989961 & 1.245777 & 0.000000 & 1 & 0.153175 & 0.151979 & 0.141515 & 0.149216 \\
\hline-2.892828 & -2.475424 & 0.000000 & 1 & 0.163786 & 0.161774 & 0.150439 & 0.158647 \\
\hline-4.989961 & -1.245779 & 0.000000 & 1 & 0.153175 & 0.151979 & 0.141515 & 0.149216 \\
\hline
\end{tabular}




\begin{tabular}{|c|c|c|c|c|c|c|c|}
\hline \multicolumn{8}{|c|}{ Benzo(b)fluoranthene } \\
\hline \multicolumn{4}{|c|}{ Atom Orientation } & \multicolumn{4}{|c|}{ Electrostatic Potential Charges } \\
\hline \multicolumn{3}{|c|}{ Coordinates $(\mathrm{x}, \mathrm{y}, \mathrm{z})$} & Mass & $\begin{array}{l}\text { b3lyp/6- } \\
31+G(d)\end{array}$ & $\begin{array}{c}\text { b3lyp/6- } \\
31++G(d, p)\end{array}$ & $\begin{array}{l}\text { b3lyp/cc- } \\
\text { pvdz }\end{array}$ & $\begin{array}{l}\text { b3lyp/cc- } \\
\text { pvtz }\end{array}$ \\
\hline-4.360667 & -1.644471 & 0.000000 & 12 & -0.147232 & -0.145233 & -0.134480 & -0.136029 \\
\hline-3.058663 & -2.091313 & 0.000000 & 12 & -0.076495 & -0.077721 & -0.052668 & -0.093147 \\
\hline-1.963847 & -1.174449 & 0.000000 & 12 & -0.020687 & -0.013328 & -0.028023 & 0.028588 \\
\hline-2.231907 & 0.248739 & 0.000000 & 12 & 0.227948 & 0.221301 & 0.176102 & 0.183903 \\
\hline-3.561786 & 0.662107 & 0.000000 & 12 & -0.216305 & -0.212000 & -0.175736 & -0.198066 \\
\hline-4.610443 & -0.263513 & 0.000000 & 12 & 0.005736 & 0.006467 & 0.014705 & 0.008459 \\
\hline-0.647054 & -1.677001 & 0.000000 & 12 & -0.017066 & -0.021499 & -0.018421 & -0.057361 \\
\hline-1.105231 & 1.179013 & 0.000000 & 12 & -0.121898 & -0.118130 & -0.081995 & -0.077430 \\
\hline 0.169825 & 0.613264 & 0.000000 & 12 & 0.258466 & 0.257755 & 0.155499 & 0.211899 \\
\hline 0.435083 & -0.785803 & 0.000000 & 12 & -0.125218 & -0.124318 & -0.033018 & -0.077615 \\
\hline 1.376956 & 1.345556 & 0.000000 & 12 & -0.181971 & -0.182486 & -0.110995 & -0.146356 \\
\hline 1.310157 & 2.724113 & 0.000000 & 12 & -0.060628 & -0.060613 & -0.065762 & -0.076826 \\
\hline 0.031042 & 3.325435 & 0.000001 & 12 & -0.105114 & -0.100439 & -0.082076 & -0.078075 \\
\hline-1.151943 & 2.587217 & 0.000000 & 12 & -0.113773 & -0.116052 & -0.101885 & -0.138236 \\
\hline-0.491854 & -2.750462 & 0.000000 & 1 & 0.146420 & 0.147102 & 0.139035 & 0.149476 \\
\hline-5.184298 & -2.346861 & 0.000000 & 1 & 0.166736 & 0.165186 & 0.155450 & 0.161743 \\
\hline-2.841822 & -3.153570 & 0.000000 & 1 & 0.150919 & 0.150144 & 0.138900 & 0.151029 \\
\hline-3.799909 & 1.718033 & 0.000000 & 1 & 0.169156 & 0.167369 & 0.153830 & 0.162024 \\
\hline-5.632474 & 0.097110 & 0.000000 & 1 & 0.151879 & 0.150453 & 0.140356 & 0.146904 \\
\hline 2.196816 & 3.346395 & 0.000000 & 1 & 0.155152 & 0.154285 & 0.145847 & 0.153309 \\
\hline-0.033371 & 4.407233 & 0.000001 & 1 & 0.158634 & 0.156403 & 0.145771 & 0.150498 \\
\hline-2.095971 & 3.116898 & 0.000001 & 1 & 0.153464 & 0.153112 & 0.142410 & 0.154792 \\
\hline 2.468575 & 0.342819 & 0.000000 & 12 & 0.136418 & 0.136417 & 0.101052 & 0.123046 \\
\hline 1.871985 & -0.957288 & 0.000000 & 12 & 0.141806 & 0.141715 & 0.066083 & 0.118964 \\
\hline 3.839693 & 0.468098 & 0.000000 & 12 & -0.162188 & -0.161175 & -0.133101 & -0.153358 \\
\hline 2.661942 & -2.110215 & 0.000001 & 12 & -0.123341 & -0.122792 & -0.081765 & -0.116154 \\
\hline 4.631565 & -0.706252 & 0.000000 & 12 & -0.023241 & -0.021492 & -0.013815 & -0.020232 \\
\hline 4.319691 & 1.439484 & 0.000000 & 1 & 0.165396 & 0.164332 & 0.152127 & 0.160003 \\
\hline 4.054249 & -1.971805 & 0.000000 & 12 & -0.154846 & -0.151545 & -0.140720 & -0.143908 \\
\hline 2.211419 & -3.096062 & 0.000001 & 1 & 0.159054 & 0.158053 & 0.143712 & 0.154797 \\
\hline 5.710959 & -0.611093 & 0.000000 & 1 & 0.142267 & 0.140451 & 0.133714 & 0.138615 \\
\hline 4.683632 & -2.852613 & 0.000000 & 1 & 0.160551 & 0.158278 & 0.149867 & 0.154744 \\
\hline
\end{tabular}




\begin{tabular}{|c|c|c|c|c|c|c|c|}
\hline \multicolumn{8}{|c|}{ Benzo(k)fluoranthene } \\
\hline \multicolumn{4}{|c|}{ Atom Orientation } & \multicolumn{4}{|c|}{ Electrostatic Potential Charges } \\
\hline \multicolumn{3}{|c|}{ Coordinates $(\mathrm{x}, \mathrm{y}, \mathrm{z})$} & \multirow{2}{*}{$\begin{array}{c}\text { Mass } \\
12 \\
\end{array}$} & \multirow{2}{*}{$\begin{array}{c}\text { b3lyp/6- } \\
31+G(d) \\
-0.110455\end{array}$} & \multirow{2}{*}{$\begin{array}{c}\begin{array}{c}\text { b3lyp/6- } \\
31++G(d, p)\end{array} \\
-0.085483 \\
\end{array}$} & \multirow{2}{*}{$\begin{array}{c}\begin{array}{c}\text { b3lyp/cc- } \\
\text { pvdz }\end{array} \\
-0.094517 \\
\end{array}$} & \multirow{2}{*}{$\begin{array}{c}\begin{array}{c}\text { b3lyp/cc- } \\
\text { pvtz }\end{array} \\
-0.072489 \\
\end{array}$} \\
\hline 0.000000 & 2.435910 & 3.273551 & & & & & \\
\hline 0.000000 & 1.272549 & 4.045102 & 12 & -0.126911 & -0.144054 & -0.096401 & -0.150578 \\
\hline 0.000000 & 2.407246 & 1.867231 & 12 & -0.097480 & -0.129897 & -0.091865 & -0.130109 \\
\hline 0.000000 & 0.000000 & 3.419236 & 12 & 0.100168 & 0.091488 & 0.048101 & 0.110847 \\
\hline 0.000000 & 0.000000 & 2.017545 & 12 & 0.115130 & 0.142156 & 0.116489 & 0.112406 \\
\hline 0.000000 & 1.169349 & 1.224059 & 12 & -0.013349 & 0.043746 & 0.024220 & 0.043871 \\
\hline 0.000000 & -1.169349 & 1.224059 & 12 & -0.013349 & 0.043745 & 0.024219 & 0.043871 \\
\hline 0.000000 & -2.407246 & 1.867231 & 12 & -0.097480 & -0.129897 & -0.091864 & -0.130109 \\
\hline 0.000000 & -2.435910 & 3.273551 & 12 & -0.110455 & -0.085484 & -0.094518 & -0.072489 \\
\hline 0.000000 & -1.272549 & 4.045102 & 12 & -0.126911 & -0.144054 & -0.096401 & -0.150579 \\
\hline 0.000000 & 3.338434 & 1.312810 & 1 & 0.157264 & 0.160641 & 0.147509 & 0.158576 \\
\hline 0.000000 & -3.338434 & 1.312810 & 1 & 0.157264 & 0.160641 & 0.147509 & 0.158576 \\
\hline 0.000000 & -3.396006 & 3.775253 & 1 & 0.156618 & 0.149800 & 0.144697 & 0.145902 \\
\hline 0.000000 & -1.345981 & 5.126815 & 1 & 0.162377 & 0.166080 & 0.151199 & 0.164379 \\
\hline 0.000000 & -0.721617 & -0.163977 & 12 & 0.069314 & -0.023375 & -0.016261 & -0.004954 \\
\hline 0.000000 & 0.721617 & -0.163977 & 12 & 0.069314 & -0.023375 & -0.016261 & -0.004953 \\
\hline 0.000000 & -1.419888 & -1.354378 & 12 & -0.221112 & -0.102795 & -0.067710 & -0.122662 \\
\hline 0.000000 & 1.419888 & -1.354378 & 12 & -0.221112 & -0.102795 & -0.067711 & -0.122662 \\
\hline 0.000000 & -0.716225 & -2.595672 & 12 & 0.156412 & 0.088022 & 0.049623 & 0.098719 \\
\hline 0.000000 & -2.504711 & -1.366889 & 1 & 0.181288 & 0.157888 & 0.144870 & 0.158209 \\
\hline 0.000000 & 0.716225 & -2.595672 & 12 & 0.156412 & 0.088021 & 0.049623 & 0.098719 \\
\hline 0.000000 & 2.504711 & -1.366889 & 1 & 0.181288 & 0.157888 & 0.144870 & 0.158209 \\
\hline 0.000000 & -1.398524 & -3.825520 & 12 & -0.147554 & -0.121179 & -0.089094 & -0.124563 \\
\hline 0.000000 & 1.398524 & -3.825520 & 12 & -0.147554 & -0.121179 & -0.089094 & -0.124563 \\
\hline 0.000000 & -0.698041 & -5.030544 & 12 & -0.081824 & -0.087772 & -0.076277 & -0.083144 \\
\hline 0.000000 & -2.482836 & -3.829265 & 1 & 0.153711 & 0.152869 & 0.139913 & 0.151620 \\
\hline 0.000000 & 0.698041 & -5.030544 & 12 & -0.081824 & -0.087773 & -0.076278 & -0.083144 \\
\hline 0.000000 & 2.482836 & -3.829265 & 1 & 0.153711 & 0.152869 & 0.139913 & 0.151620 \\
\hline 0.000000 & -1.240139 & -5.968174 & 1 & 0.154052 & 0.158690 & 0.147801 & 0.155595 \\
\hline 0.000000 & 1.240139 & -5.968174 & 1 & 0.154052 & 0.158690 & 0.147801 & 0.155595 \\
\hline 0.000000 & 1.345981 & 5.126815 & 1 & 0.162377 & 0.166080 & 0.151199 & 0.164379 \\
\hline 0.000000 & 3.396006 & 3.775253 & 1 & 0.156618 & 0.149800 & 0.144697 & 0.145902 \\
\hline
\end{tabular}




\begin{tabular}{|c|c|c|c|c|c|c|c|}
\hline \multicolumn{8}{|c|}{ Perylene } \\
\hline \multicolumn{4}{|c|}{ Atom Orientation } & \multicolumn{4}{|c|}{ Electrostatic Potential Charges } \\
\hline \multicolumn{3}{|c|}{ Coordinates $(\mathrm{x}, \mathrm{y}, \mathrm{z})$} & \multirow{2}{*}{$\begin{array}{c}\text { Mass } \\
12 \\
\end{array}$} & \multirow{2}{*}{$\begin{array}{c}\text { b3lyp/6- } \\
31+G(d) \\
-0.002022 \\
\end{array}$} & \multirow{2}{*}{$\begin{array}{c}\begin{array}{c}\text { b3lyp/6- } \\
31++\mathrm{G}(\mathrm{d}, \mathrm{p})\end{array} \\
-0.009478 \\
\end{array}$} & \multirow{2}{*}{$\begin{array}{c}\begin{array}{c}\text { b3lyp/cc- } \\
\text { pvdz }\end{array} \\
0.009164 \\
\end{array}$} & \multirow{2}{*}{$\begin{array}{c}\begin{array}{c}\text { b3lyp/cc- } \\
\text { pvtz }\end{array} \\
0.020930 \\
\end{array}$} \\
\hline 0.738169 & 1.249810 & 0.000000 & & & & & \\
\hline-0.738171 & 1.249819 & 0.000000 & 12 & -0.006527 & 0.007243 & -0.004032 & 0.009207 \\
\hline-1.439330 & -0.000011 & 0.000000 & 12 & 0.121748 & 0.104796 & 0.089078 & 0.075822 \\
\hline-0.738179 & -1.249821 & 0.000000 & 12 & -0.002518 & 0.007244 & -0.004031 & 0.009207 \\
\hline 0.738171 & -1.249820 & 0.000000 & 12 & -0.006005 & -0.009478 & 0.009164 & 0.020930 \\
\hline 1.439330 & 0.000001 & 0.000000 & 12 & 0.104725 & 0.105815 & 0.087772 & 0.075328 \\
\hline 1.479612 & -2.427539 & 0.000010 & 12 & -0.085789 & -0.087539 & -0.082497 & -0.109376 \\
\hline 2.874760 & 0.000002 & 0.000000 & 12 & 0.155117 & 0.160962 & 0.075307 & 0.151190 \\
\hline 2.885922 & -2.422668 & 0.000020 & 12 & -0.086937 & -0.082204 & -0.080234 & -0.062071 \\
\hline 0.977182 & -3.388099 & 0.000020 & 1 & 0.143136 & 0.143025 & 0.135978 & 0.142455 \\
\hline 3.575551 & -1.232477 & 0.000010 & 12 & -0.164057 & -0.167639 & -0.102746 & -0.159390 \\
\hline 3.421382 & -3.368167 & 0.000020 & 1 & 0.151485 & 0.150379 & 0.142282 & 0.144180 \\
\hline 4.662511 & -1.217906 & 0.000010 & 1 & 0.167628 & 0.167359 & 0.148646 & 0.162032 \\
\hline 3.575539 & 1.232483 & -0.000010 & 12 & -0.165293 & -0.167639 & -0.102746 & -0.159391 \\
\hline 2.885898 & 2.422682 & -0.000020 & 12 & -0.085473 & -0.082204 & -0.080234 & -0.062071 \\
\hline 4.662499 & 1.217924 & -0.000010 & 1 & 0.167874 & 0.167359 & 0.148646 & 0.162032 \\
\hline 1.479598 & 2.427541 & -0.000020 & 12 & -0.088706 & -0.087538 & -0.082497 & -0.109377 \\
\hline 3.421357 & 3.368183 & -0.000030 & 1 & 0.151350 & 0.150379 & 0.142282 & 0.144180 \\
\hline 0.977147 & 3.388091 & -0.000020 & 1 & 0.143750 & 0.143025 & 0.135978 & 0.142455 \\
\hline-1.479608 & -2.427551 & -0.000010 & 12 & -0.090333 & -0.098499 & -0.073815 & -0.102438 \\
\hline-2.874760 & -0.000003 & 0.000000 & 12 & 0.120602 & 0.137207 & 0.092228 & 0.170323 \\
\hline-2.885908 & -2.422673 & -0.000020 & 12 & -0.085085 & -0.075959 & -0.085339 & -0.064828 \\
\hline-0.977168 & -3.388091 & -0.000020 & 1 & 0.140372 & 0.141684 & 0.136987 & 0.143241 \\
\hline-3.575539 & -1.232473 & -0.000010 & 12 & -0.146402 & -0.154655 & -0.111666 & -0.171048 \\
\hline-3.421378 & -3.368173 & -0.000020 & 1 & 0.149853 & 0.147907 & 0.144090 & 0.145796 \\
\hline-4.662509 & -1.217924 & -0.000010 & 1 & 0.163560 & 0.163985 & 0.150991 & 0.164980 \\
\hline-3.575541 & 1.232487 & 0.000010 & 12 & -0.145177 & -0.154655 & -0.111666 & -0.171048 \\
\hline-2.885912 & 2.422667 & 0.000020 & 12 & -0.086549 & -0.075959 & -0.085339 & -0.064828 \\
\hline-4.662511 & 1.217916 & 0.000010 & 1 & 0.163318 & 0.163985 & 0.150991 & 0.164980 \\
\hline-1.479592 & 2.427539 & 0.000020 & 12 & -0.087379 & -0.098498 & -0.073816 & -0.102438 \\
\hline-3.421363 & 3.368177 & 0.000030 & 1 & 0.149983 & 0.147907 & 0.144090 & 0.145796 \\
\hline-0.977163 & 3.388089 & 0.000020 & 1 & 0.139752 & 0.141684 & 0.136987 & 0.143241 \\
\hline
\end{tabular}




\begin{tabular}{|c|c|c|c|c|c|c|c|}
\hline \multicolumn{8}{|c|}{ Benzo(g,h,i)perylene } \\
\hline \multicolumn{4}{|c|}{ Atom Orientation } & \multicolumn{4}{|c|}{ Electrostatic Potential Charges } \\
\hline \multicolumn{3}{|c|}{ Coordinates $(\mathrm{x}, \mathrm{y}, \mathrm{z})$} & Mass & $\begin{array}{l}\text { b3lyp/6- } \\
31+G(d)\end{array}$ & $\begin{array}{c}\text { b3lyp/6- } \\
31++\mathrm{G}(\mathrm{d}, \mathrm{p})\end{array}$ & $\begin{array}{l}\text { b3lyp/cc- } \\
\text { pvdz }\end{array}$ & $\begin{array}{l}\text { b3lyp/cc- } \\
\text { pvtz }\end{array}$ \\
\hline 0.000000 & 0.707729 & 0.893208 & 12 & -0.076290 & -0.075752 & -0.056792 & -0.064862 \\
\hline 0.000000 & -0.707729 & 0.893208 & 12 & -0.076290 & -0.075752 & -0.056792 & -0.064862 \\
\hline 0.000000 & -1.420312 & -0.340910 & 12 & 0.108300 & 0.104311 & 0.090456 & 0.082882 \\
\hline 0.000000 & -0.723561 & -1.583487 & 12 & 0.027888 & 0.030194 & 0.022031 & 0.043165 \\
\hline 0.000000 & 0.723561 & -1.583487 & 12 & 0.027888 & 0.030194 & 0.022031 & 0.043165 \\
\hline 0.000000 & 1.420312 & -0.340910 & 12 & 0.108300 & 0.104312 & 0.090456 & 0.082882 \\
\hline 0.000000 & 1.487431 & -2.779333 & 12 & -0.125773 & -0.128562 & -0.100418 & -0.139982 \\
\hline 0.000000 & 2.845812 & -0.322244 & 12 & 0.087508 & 0.092459 & 0.046940 & 0.101287 \\
\hline 0.000000 & 2.874927 & -2.756873 & 12 & -0.099598 & -0.092311 & -0.097855 & -0.077093 \\
\hline 0.000000 & 0.990392 & -3.739395 & 1 & 0.154875 & 0.154497 & 0.144998 & 0.154808 \\
\hline 0.000000 & 3.555441 & -1.540762 & 12 & -0.100637 & -0.104623 & -0.064334 & -0.110846 \\
\hline 0.000000 & 3.428039 & -3.687810 & 1 & 0.152579 & 0.149782 & 0.144468 & 0.145851 \\
\hline 0.000000 & 4.639556 & -1.522321 & 1 & 0.149494 & 0.149168 & 0.138011 & 0.148201 \\
\hline 0.000000 & 3.525205 & 0.927493 & 12 & -0.123807 & -0.124533 & -0.090384 & -0.125467 \\
\hline 0.000000 & 2.830493 & 2.118770 & 12 & -0.224875 & -0.222582 & -0.183349 & -0.206223 \\
\hline 0.000000 & 4.609441 & 0.932124 & 1 & 0.166384 & 0.165799 & 0.151285 & 0.163196 \\
\hline 0.000000 & 1.417412 & 2.133874 & 12 & 0.265710 & 0.264672 & 0.209862 & 0.247119 \\
\hline 0.000000 & 3.367860 & 3.060169 & 1 & 0.175601 & 0.174280 & 0.159945 & 0.168948 \\
\hline 0.000000 & -1.487431 & -2.779333 & 12 & -0.125773 & -0.128562 & -0.100418 & -0.139982 \\
\hline 0.000000 & -2.845812 & -0.322244 & 12 & 0.087508 & 0.092459 & 0.046940 & 0.101287 \\
\hline 0.000000 & -2.874927 & -2.756873 & 12 & -0.099598 & -0.092311 & -0.097854 & -0.077093 \\
\hline 0.000000 & -0.990392 & -3.739395 & 1 & 0.154875 & 0.154497 & 0.144998 & 0.154808 \\
\hline 0.000000 & -3.555441 & -1.540762 & 12 & -0.100637 & -0.104623 & -0.064334 & -0.110846 \\
\hline 0.000000 & -3.428039 & -3.687810 & 1 & 0.152579 & 0.149782 & 0.144468 & 0.145851 \\
\hline 0.000000 & -4.639556 & -1.522321 & 1 & 0.149494 & 0.149168 & 0.138011 & 0.148201 \\
\hline 0.000000 & -3.525205 & 0.927493 & 12 & -0.123807 & -0.124533 & -0.090384 & -0.125467 \\
\hline 0.000000 & -2.830493 & 2.118770 & 12 & -0.224875 & -0.222582 & -0.183349 & -0.206223 \\
\hline 0.000000 & -4.609441 & 0.932124 & 1 & 0.166384 & 0.165799 & 0.151285 & 0.163196 \\
\hline 0.000000 & -1.417412 & 2.133874 & 12 & 0.265710 & 0.264672 & 0.209862 & 0.247119 \\
\hline 0.000000 & -3.367860 & 3.060169 & 1 & 0.175601 & 0.174280 & 0.159945 & 0.168948 \\
\hline 0.000000 & -0.682634 & 3.360630 & 12 & -0.211540 & -0.209662 & -0.174971 & -0.199723 \\
\hline 0.000000 & 0.682634 & 3.360630 & 12 & -0.211540 & -0.209662 & -0.174971 & -0.199723 \\
\hline 0.000000 & -1.231869 & 4.295037 & 1 & 0.174180 & 0.172863 & 0.160107 & 0.168739 \\
\hline 0.000000 & 1.231869 & 4.295037 & 1 & 0.174180 & 0.172863 & 0.160107 & 0.168739 \\
\hline
\end{tabular}




\begin{tabular}{|c|c|c|c|c|c|c|c|}
\hline \multicolumn{8}{|c|}{ Indeno(1,2,3-cd)pyrene } \\
\hline \multicolumn{4}{|c|}{ Atom Orientation } & \multicolumn{4}{|c|}{ Electrostatic Potential Charges } \\
\hline \multicolumn{3}{|c|}{ Coordinates $(\mathrm{x}, \mathrm{y}, \mathrm{z})$} & \multirow{2}{*}{$\begin{array}{c}\text { Mass } \\
12 \\
\end{array}$} & \multirow{2}{*}{$\begin{array}{c}\text { b3lyp/6- } \\
31+G(d) \\
-0.173392 \\
\end{array}$} & \multirow{2}{*}{$\begin{array}{c}\begin{array}{c}\text { b3lyp/6- } \\
31++\mathrm{G}(\mathrm{d}, \mathrm{p})\end{array} \\
-0.172915 \\
\end{array}$} & \multirow{2}{*}{$\begin{array}{c}\begin{array}{c}\text { b3lyp/cc- } \\
\text { pvdz }\end{array} \\
-0.124664\end{array}$} & \multirow{2}{*}{$\begin{array}{c}\begin{array}{c}\text { b3lyp/cc- } \\
\text { pvtz }\end{array} \\
-0.101851\end{array}$} \\
\hline-2.400937 & 1.870922 & 0.000000 & & & & & \\
\hline 0.000000 & 0.524190 & 0.000000 & 12 & 0.322586 & 0.324939 & 0.228237 & 0.058845 \\
\hline 0.010670 & 1.950039 & 0.000000 & 12 & -0.145856 & -0.148120 & -0.053361 & 0.128940 \\
\hline-1.226943 & 2.617629 & 0.000000 & 12 & -0.078982 & -0.076414 & -0.100134 & -0.195521 \\
\hline-3.353775 & 2.389093 & 0.000000 & 1 & 0.168587 & 0.167527 & 0.153949 & 0.159536 \\
\hline-1.283196 & 3.699254 & 0.000000 & 1 & 0.163190 & 0.162130 & 0.156018 & 0.175679 \\
\hline-1.158148 & -0.233379 & 0.000000 & 12 & -0.192421 & -0.192292 & -0.150492 & -0.036385 \\
\hline-1.032069 & -1.636910 & 0.000000 & 12 & 0.175678 & 0.168638 & 0.134772 & 0.040625 \\
\hline-2.410218 & 0.435275 & 0.000000 & 12 & 0.229806 & 0.230053 & 0.177893 & 0.125856 \\
\hline-2.212352 & -2.425971 & 0.000000 & 12 & -0.024105 & -0.014969 & -0.023762 & 0.082946 \\
\hline-3.571090 & -0.370244 & 0.000000 & 12 & -0.206489 & -0.204221 & -0.165676 & -0.146168 \\
\hline-3.468261 & -1.756535 & 0.000000 & 12 & -0.047229 & -0.050274 & -0.034491 & -0.104539 \\
\hline-4.550948 & 0.093580 & 0.000000 & 1 & 0.173706 & 0.172619 & 0.157793 & 0.161063 \\
\hline-4.373873 & -2.353228 & 0.000000 & 1 & 0.153383 & 0.153288 & 0.141627 & 0.158012 \\
\hline 1.304766 & -0.020311 & 0.000000 & 12 & -0.229733 & -0.229351 & -0.143021 & 0.063871 \\
\hline 1.440296 & -1.392533 & 0.000000 & 12 & -0.077424 & -0.079905 & -0.072052 & -0.224556 \\
\hline 2.413646 & -1.871174 & 0.000000 & 1 & 0.159562 & 0.159889 & 0.149612 & 0.174870 \\
\hline 0.266936 & -2.223292 & 0.000000 & 12 & 0.013354 & 0.018787 & -0.003047 & 0.107445 \\
\hline 0.338849 & -3.634946 & 0.000000 & 12 & -0.083621 & -0.085817 & -0.046042 & -0.094789 \\
\hline-0.815862 & -4.419101 & 0.000000 & 12 & -0.150743 & -0.144311 & -0.142569 & -0.127454 \\
\hline 1.311250 & -4.115291 & 0.000000 & 1 & 0.156960 & 0.156102 & 0.141208 & 0.153805 \\
\hline-2.078174 & -3.833922 & 0.000000 & 12 & -0.051465 & -0.057819 & -0.031439 & -0.102658 \\
\hline-0.725968 & -5.498239 & 0.000000 & 1 & 0.168221 & 0.165941 & 0.156690 & 0.162791 \\
\hline-2.966034 & -4.456252 & 0.000000 & 1 & 0.150645 & 0.150873 & 0.139174 & 0.156130 \\
\hline 1.409120 & 2.339274 & 0.000000 & 12 & 0.117349 & 0.116635 & 0.059138 & -0.036052 \\
\hline 2.009064 & 3.600640 & 0.000000 & 12 & -0.134468 & -0.132439 & -0.094996 & -0.077511 \\
\hline 2.214081 & 1.150939 & 0.000000 & 12 & 0.218237 & 0.216598 & 0.144650 & 0.073361 \\
\hline 3.403131 & 3.683400 & 0.000000 & 12 & -0.154147 & -0.150980 & -0.138151 & -0.131385 \\
\hline 1.407732 & 4.502183 & 0.000000 & 1 & 0.162251 & 0.160826 & 0.146685 & 0.147972 \\
\hline 3.589649 & 1.246447 & 0.000000 & 12 & -0.247831 & -0.244026 & -0.191881 & -0.164329 \\
\hline 4.180715 & 2.525954 & 0.000000 & 12 & -0.008582 & -0.008292 & -0.007212 & -0.050365 \\
\hline 3.884135 & 4.653532 & 0.000000 & 1 & 0.158145 & 0.155844 & 0.147540 & 0.154127 \\
\hline 4.216645 & 0.362676 & 0.000000 & 1 & 0.176967 & 0.175228 & 0.158147 & 0.159339 \\
\hline 5.261052 & 2.608476 & 0.000000 & 1 & 0.137861 & 0.136227 & 0.129859 & 0.148350 \\
\hline
\end{tabular}




\begin{tabular}{|c|c|c|c|c|c|c|c|}
\hline \multicolumn{8}{|c|}{ 1,2,3,4- Dibenzanthracene } \\
\hline \multicolumn{4}{|c|}{ Atom Orientation } & \multicolumn{4}{|c|}{ Electrostatic Potential Charges } \\
\hline \multicolumn{3}{|c|}{ Coordinates $(\mathrm{x}, \mathrm{y}, \mathrm{z})$} & Mass & $\begin{array}{l}\text { b3lyp/6- } \\
31+G(d)\end{array}$ & $\begin{array}{c}\text { b3lyp/6- } \\
31++G(d, p)\end{array}$ & $\begin{array}{l}\text { b3lyp/cc- } \\
\text { pvdz }\end{array}$ & $\begin{array}{c}\text { b3lyp/cc- } \\
\text { pvtz }\end{array}$ \\
\hline-5.142409 & 0.701237 & 0.000000 & 12 & -0.078458 & -0.077352 & -0.066506 & -0.072391 \\
\hline-3.941110 & 1.404273 & 0.000002 & 12 & -0.136913 & -0.136538 & -0.102632 & -0.140504 \\
\hline-2.710671 & 0.713356 & 0.000000 & 12 & 0.094295 & 0.096662 & 0.054814 & 0.106240 \\
\hline-2.710671 & -0.713356 & -0.000002 & 12 & 0.094297 & 0.096662 & 0.054814 & 0.106240 \\
\hline-3.941110 & -1.404273 & -0.000003 & 12 & -0.136914 & -0.136538 & -0.102632 & -0.140504 \\
\hline-5.142409 & -0.701237 & -0.000002 & 12 & -0.078458 & -0.077352 & -0.066506 & -0.072391 \\
\hline-1.468173 & 1.384434 & 0.000002 & 12 & -0.106663 & -0.108497 & -0.063464 & -0.119125 \\
\hline-1.468173 & -1.384434 & -0.000002 & 12 & -0.106665 & -0.108497 & -0.063464 & -0.119125 \\
\hline-0.234339 & -0.716536 & -0.000001 & 12 & 0.017220 & 0.016714 & 0.008532 & 0.017910 \\
\hline-0.234339 & 0.716536 & 0.000000 & 12 & 0.017215 & 0.016714 & 0.008532 & 0.017910 \\
\hline 1.021795 & 1.448674 & 0.000000 & 12 & 0.083519 & 0.086040 & 0.062314 & 0.097728 \\
\hline 2.260942 & 0.725421 & 0.000000 & 12 & 0.078326 & 0.075283 & 0.067409 & 0.071192 \\
\hline 2.260942 & -0.725421 & 0.000000 & 12 & 0.078331 & 0.075283 & 0.067409 & 0.071192 \\
\hline 1.021795 & -1.448674 & -0.000001 & 12 & 0.083514 & 0.086040 & 0.062314 & 0.097728 \\
\hline-1.495939 & 2.465492 & 0.000004 & 1 & 0.139386 & 0.139365 & 0.129415 & 0.139097 \\
\hline-6.082096 & 1.240041 & 0.000000 & 1 & 0.158696 & 0.157295 & 0.146772 & 0.154198 \\
\hline-3.944516 & 2.488597 & 0.000004 & 1 & 0.162603 & 0.161677 & 0.146873 & 0.160330 \\
\hline-3.944516 & -2.488597 & -0.000005 & 1 & 0.162603 & 0.161677 & 0.146873 & 0.160330 \\
\hline-6.082096 & -1.240041 & -0.000004 & 1 & 0.158696 & 0.157295 & 0.146772 & 0.154198 \\
\hline-1.495939 & -2.465492 & -0.000004 & 1 & 0.139387 & 0.139365 & 0.129415 & 0.139097 \\
\hline 3.469609 & -1.464648 & 0.000000 & 12 & -0.178279 & -0.173928 & -0.154639 & -0.173280 \\
\hline 3.473855 & -2.841630 & 0.000000 & 12 & -0.087314 & -0.085986 & -0.073370 & -0.076903 \\
\hline 4.418799 & -0.950548 & 0.000002 & 1 & 0.155879 & 0.153565 & 0.144576 & 0.150593 \\
\hline 1.061888 & -2.862534 & -0.000001 & 12 & -0.195425 & -0.194163 & -0.169910 & -0.202387 \\
\hline 2.256275 & -3.549620 & -0.000001 & 12 & -0.077364 & -0.075726 & -0.060544 & -0.069135 \\
\hline 4.413969 & -3.379788 & 0.000000 & 1 & 0.158035 & 0.156353 & 0.144944 & 0.151562 \\
\hline 0.146247 & -3.435788 & -0.000001 & 1 & 0.159099 & 0.157460 & 0.145193 & 0.156748 \\
\hline 2.257562 & -4.632857 & -0.000001 & 1 & 0.153358 & 0.151775 & 0.140220 & 0.148127 \\
\hline 4.418799 & 0.950548 & -0.000002 & 1 & 0.155878 & 0.153565 & 0.144576 & 0.150593 \\
\hline 3.469609 & 1.464648 & 0.000000 & 12 & -0.178276 & -0.173928 & -0.154639 & -0.173280 \\
\hline 3.473855 & 2.841630 & 0.000000 & 12 & -0.087315 & -0.085986 & -0.073370 & -0.076903 \\
\hline 2.256275 & 3.549620 & 0.000002 & 12 & -0.077362 & -0.075726 & -0.060544 & -0.069135 \\
\hline 4.413969 & 3.379788 & 0.000000 & 1 & 0.158035 & 0.156353 & 0.144944 & 0.151562 \\
\hline 1.061888 & 2.862534 & 0.000002 & 12 & -0.195427 & -0.194163 & -0.169910 & -0.202387 \\
\hline 2.257562 & 4.632857 & 0.000003 & 1 & 0.153358 & 0.151775 & 0.140220 & 0.148127 \\
\hline 0.146247 & 3.435788 & 0.000003 & 1 & 0.159100 & 0.157460 & 0.145193 & 0.156748 \\
\hline
\end{tabular}




\begin{tabular}{|c|c|c|c|c|c|c|c|}
\hline \multicolumn{8}{|c|}{ 1,2:5,6- Dibenzanthracene } \\
\hline \multicolumn{4}{|c|}{ Atom Orientation } & \multicolumn{4}{|c|}{ Electrostatic Potential Charges } \\
\hline \multicolumn{3}{|c|}{ Coordinates $(\mathrm{x}, \mathrm{y}, \mathrm{z})$} & \multirow{2}{*}{$\begin{array}{c}\text { Mass } \\
12 \\
\end{array}$} & \multirow{2}{*}{$\begin{array}{c}\text { b3lyp/6- } \\
31+G(d) \\
0.050393\end{array}$} & \multirow{2}{*}{$\begin{array}{c}\begin{array}{c}\text { b3lyp/6- } \\
31++\mathrm{G}(\mathrm{d}, \mathrm{p})\end{array} \\
0.206579 \\
\end{array}$} & \multirow{2}{*}{$\begin{array}{c}\begin{array}{c}\text { b3lyp/cc- } \\
\text { pvdz }\end{array} \\
0.144635 \\
\end{array}$} & \multirow{2}{*}{$\begin{array}{c}\begin{array}{c}\text { b3lyp/cc- } \\
\text { pvtz }\end{array} \\
0.185842 \\
\end{array}$} \\
\hline-0.759840 & 3.707453 & 0.000000 & & & & & \\
\hline 0.406771 & 2.874334 & 0.000000 & 12 & 0.164992 & 0.128817 & 0.105220 & 0.130065 \\
\hline 0.229444 & 1.427645 & 0.000000 & 12 & -0.019951 & -0.035900 & -0.028757 & -0.031572 \\
\hline-1.095624 & 0.879662 & 0.000000 & 12 & 0.142587 & 0.225169 & 0.170544 & 0.211522 \\
\hline-2.212376 & 1.743383 & 0.000000 & 12 & -0.188439 & -0.189842 & -0.158049 & -0.183369 \\
\hline-2.045408 & 3.113555 & 0.000000 & 12 & -0.068487 & -0.179471 & -0.122219 & -0.163195 \\
\hline 1.285877 & 0.525804 & 0.000000 & 12 & -0.123963 & -0.197724 & -0.152038 & -0.186939 \\
\hline-1.285877 & -0.525805 & 0.000000 & 12 & -0.156218 & -0.207448 & -0.163404 & -0.195850 \\
\hline-0.229443 & -1.427645 & 0.000000 & 12 & 0.025486 & -0.008075 & -0.004137 & -0.010657 \\
\hline 1.095625 & -0.879662 & 0.000000 & 12 & 0.101141 & 0.217726 & 0.159842 & 0.205574 \\
\hline 2.212376 & -1.743384 & 0.000000 & 12 & -0.158888 & -0.182291 & -0.149953 & -0.178774 \\
\hline 3.209791 & -1.319057 & 0.000000 & 1 & 0.167097 & 0.172506 & 0.155748 & 0.169411 \\
\hline 2.045408 & -3.113555 & 0.000000 & 12 & -0.110261 & -0.201445 & -0.139358 & -0.178094 \\
\hline 0.759840 & -3.707453 & 0.000000 & 12 & 0.118366 & 0.238544 & 0.168305 & 0.209230 \\
\hline-0.406770 & -2.874334 & 0.000000 & 12 & 0.105887 & 0.088286 & 0.075198 & 0.100356 \\
\hline 2.308548 & 0.881641 & 0.000000 & 1 & 0.138379 & 0.150333 & 0.142683 & 0.145227 \\
\hline-3.209791 & 1.319057 & 0.000000 & 1 & 0.168906 & 0.174399 & 0.157503 & 0.170518 \\
\hline-2.915892 & 3.760378 & 0.000000 & 1 & 0.154368 & 0.180261 & 0.159617 & 0.174020 \\
\hline-2.308547 & -0.881642 & 0.000000 & 1 & 0.150655 & 0.148255 & 0.141854 & 0.144789 \\
\hline 2.915892 & -3.760379 & 0.000000 & 1 & 0.162637 & 0.183304 & 0.161910 & 0.175967 \\
\hline-1.662427 & -3.502682 & 0.000000 & 12 & -0.213595 & -0.199272 & -0.181180 & -0.204836 \\
\hline 0.622927 & -5.118954 & 0.000000 & 12 & -0.174249 & -0.235770 & -0.189571 & -0.218527 \\
\hline-1.769122 & -4.884368 & 0.000000 & 12 & -0.050518 & -0.055399 & -0.038004 & -0.046936 \\
\hline-2.570990 & -2.915347 & 0.000000 & 1 & 0.160566 & 0.157240 & 0.146825 & 0.155747 \\
\hline-0.622928 & -5.701225 & 0.000000 & 12 & -0.110386 & -0.051681 & -0.058994 & -0.055963 \\
\hline 1.518934 & -5.728997 & 0.000000 & 1 & 0.160761 & 0.160775 & 0.149657 & 0.156165 \\
\hline-2.751671 & -5.341348 & 0.000000 & 1 & 0.150032 & 0.134914 & 0.128002 & 0.133126 \\
\hline-0.726462 & -6.779109 & 0.000000 & 1 & 0.161236 & 0.137601 & 0.133752 & 0.137149 \\
\hline 1.662427 & 3.502683 & 0.000000 & 12 & -0.246896 & -0.216659 & -0.192254 & -0.218575 \\
\hline 1.769121 & 4.884368 & 0.000000 & 12 & -0.048784 & -0.055108 & -0.039622 & -0.045025 \\
\hline 2.570990 & 2.915348 & 0.000000 & 1 & 0.172960 & 0.156765 & 0.146069 & 0.156036 \\
\hline-0.622928 & 5.118955 & 0.000000 & 12 & -0.139039 & -0.229424 & -0.185089 & -0.212202 \\
\hline 0.622927 & 5.701225 & 0.000000 & 12 & -0.109786 & -0.046423 & -0.054367 & -0.054431 \\
\hline 2.751670 & 5.341350 & 0.000000 & 1 & 0.151719 & 0.137893 & 0.130528 & 0.135036 \\
\hline-1.518935 & 5.728996 & 0.000000 & 1 & 0.154575 & 0.157115 & 0.146967 & 0.153208 \\
\hline 0.726461 & 6.779110 & 0.000000 & 1 & 0.156716 & 0.135447 & 0.132135 & 0.135957 \\
\hline
\end{tabular}




\begin{tabular}{|c|c|c|c|c|c|c|c|}
\hline \multicolumn{8}{|c|}{ Pentacene } \\
\hline \multicolumn{4}{|c|}{ Atom Orientation } & \multicolumn{4}{|c|}{ Electrostatic Potential Charges } \\
\hline \multicolumn{3}{|c|}{ Coordinates $(\mathrm{x}, \mathrm{y}, \mathrm{z})$} & \multirow{2}{*}{$\begin{array}{c}\text { Mass } \\
12\end{array}$} & \multirow{2}{*}{$\begin{array}{c}\text { b3lyp/6- } \\
31+G(d) \\
-0.066658\end{array}$} & \multirow{2}{*}{$\begin{array}{c}\begin{array}{c}\text { b3lyp/6- } \\
31++G(d, p)\end{array} \\
-0.064654\end{array}$} & \multirow{2}{*}{$\begin{array}{c}\begin{array}{c}\text { b3lyp/cc- } \\
\text { pvdz }\end{array} \\
-0.056704\end{array}$} & \multirow{2}{*}{$\begin{array}{c}\begin{array}{c}\text { b3lyp/cc- } \\
\text { pvtz }\end{array} \\
-0.060549\end{array}$} \\
\hline 0.000000 & 0.709480 & 6.109310 & & & & & \\
\hline 0.000000 & 1.409867 & 4.925092 & 12 & -0.227195 & -0.225448 & -0.190588 & -0.222687 \\
\hline 0.000000 & 0.723161 & 3.679870 & 12 & 0.229511 & 0.229130 & 0.173839 & 0.220979 \\
\hline 0.000000 & -0.723161 & 3.679870 & 12 & 0.229511 & 0.229130 & 0.173839 & 0.220979 \\
\hline 0.000000 & -1.409867 & 4.925092 & 12 & -0.227195 & -0.225448 & -0.190588 & -0.222687 \\
\hline 0.000000 & -0.709480 & 6.109310 & 12 & -0.066658 & -0.064654 & -0.056704 & -0.060549 \\
\hline 0.000000 & 1.408514 & 2.456111 & 12 & -0.329353 & -0.328892 & -0.252344 & -0.316940 \\
\hline 0.000000 & -1.408514 & 2.456111 & 12 & -0.329353 & -0.328892 & -0.252344 & -0.316940 \\
\hline 0.000000 & -0.726869 & 1.228184 & 12 & 0.174435 & 0.174454 & 0.130317 & 0.169070 \\
\hline 0.000000 & 0.726869 & 1.228184 & 12 & 0.174435 & 0.174454 & 0.130317 & 0.169070 \\
\hline 0.000000 & 1.409939 & 0.000000 & 12 & -0.244217 & -0.243447 & -0.190919 & -0.236839 \\
\hline 0.000000 & 2.495251 & 0.000000 & 1 & 0.181000 & 0.180472 & 0.163090 & 0.176533 \\
\hline 0.000000 & 0.726869 & -1.228184 & 12 & 0.174435 & 0.174454 & 0.130317 & 0.169070 \\
\hline 0.000000 & -0.726869 & -1.228184 & 12 & 0.174435 & 0.174454 & 0.130317 & 0.169070 \\
\hline 0.000000 & -1.409939 & 0.000000 & 12 & -0.244217 & -0.243447 & -0.190919 & -0.236839 \\
\hline 0.000000 & 2.493716 & 2.458263 & 1 & 0.188604 & 0.188103 & 0.168659 & 0.184248 \\
\hline 0.000000 & 1.241430 & 7.053126 & 1 & 0.147476 & 0.145505 & 0.138422 & 0.143992 \\
\hline 0.000000 & 2.493948 & 4.927061 & 1 & 0.164789 & 0.163290 & 0.152314 & 0.162040 \\
\hline 0.000000 & -2.493948 & 4.927061 & 1 & 0.164789 & 0.163290 & 0.152314 & 0.162040 \\
\hline 0.000000 & -1.241430 & 7.053126 & 1 & 0.147476 & 0.145505 & 0.138422 & 0.143992 \\
\hline 0.000000 & -2.493716 & 2.458263 & 1 & 0.188604 & 0.188103 & 0.168659 & 0.184248 \\
\hline 0.000000 & -2.495251 & 0.000000 & 1 & 0.181000 & 0.180472 & 0.163090 & 0.176533 \\
\hline 0.000000 & -1.408514 & -2.456111 & 12 & -0.329353 & -0.328892 & -0.252344 & -0.316940 \\
\hline 0.000000 & 1.408514 & -2.456111 & 12 & -0.329353 & -0.328892 & -0.252344 & -0.316940 \\
\hline 0.000000 & -0.723161 & -3.679870 & 12 & 0.229511 & 0.229130 & 0.173839 & 0.220979 \\
\hline 0.000000 & -2.493716 & -2.458263 & 1 & 0.188604 & 0.188103 & 0.168659 & 0.184248 \\
\hline 0.000000 & 0.723161 & -3.679870 & 12 & 0.229511 & 0.229130 & 0.173839 & 0.220979 \\
\hline 0.000000 & 2.493716 & -2.458263 & 1 & 0.188604 & 0.188103 & 0.168659 & 0.184248 \\
\hline 0.000000 & -1.409867 & -4.925092 & 12 & -0.227195 & -0.225448 & -0.190588 & -0.222687 \\
\hline 0.000000 & 1.409867 & -4.925092 & 12 & -0.227195 & -0.225448 & -0.190588 & -0.222687 \\
\hline 0.000000 & -0.709480 & -6.109310 & 12 & -0.066658 & -0.064654 & -0.056704 & -0.060548 \\
\hline 0.000000 & -2.493948 & -4.927061 & 1 & 0.164789 & 0.163290 & 0.152314 & 0.162040 \\
\hline 0.000000 & 0.709480 & -6.109310 & 12 & -0.066658 & -0.064654 & -0.056704 & -0.060548 \\
\hline 0.000000 & 2.493948 & -4.927061 & 1 & 0.164789 & 0.163290 & 0.152314 & 0.162040 \\
\hline 0.000000 & -1.241430 & -7.053126 & 1 & 0.147476 & 0.145505 & 0.138422 & 0.143992 \\
\hline 0.000000 & 1.241430 & -7.053126 & 1 & 0.147476 & 0.145505 & 0.138422 & 0.143992 \\
\hline
\end{tabular}




\begin{tabular}{|c|c|c|c|c|c|c|c|}
\hline \multicolumn{8}{|c|}{ Rubrene } \\
\hline \multicolumn{4}{|c|}{ Atom Orientation } & \multicolumn{4}{|c|}{ Electrostatic Potential Charges } \\
\hline \multicolumn{3}{|c|}{ Coordinates $(\mathrm{x}, \mathrm{y}, \mathrm{z})$} & \multirow{2}{*}{$\begin{array}{c}\text { Mass } \\
12\end{array}$} & \multirow{2}{*}{$\begin{array}{c}\text { b3lyp/6- } \\
31+G(d) \\
-0.067863\end{array}$} & \multirow{2}{*}{$\begin{array}{c}\begin{array}{c}\text { b3lyp/6- } \\
31++G(d, p)\end{array} \\
-0.066729 \\
\end{array}$} & \multirow{2}{*}{$\begin{array}{c}\begin{array}{c}\text { b3lyp/cc- } \\
\text { pvdz }\end{array} \\
-0.062109\end{array}$} & \multirow{2}{*}{$\begin{array}{c}\begin{array}{c}\text { b3lyp/cc- } \\
\text { pvtz }\end{array} \\
-0.067214\end{array}$} \\
\hline 0.647301 & 4.886807 & 0.278278 & & & & & \\
\hline 1.299312 & 3.695465 & 0.517364 & 12 & -0.220926 & -0.220438 & -0.174591 & -0.211830 \\
\hline 0.686648 & 2.448096 & 0.218284 & 12 & 0.232195 & 0.234998 & 0.160740 & 0.224204 \\
\hline-0.686928 & 2.448149 & -0.217942 & 12 & 0.153070 & 0.157454 & 0.094708 & 0.157122 \\
\hline-1.299502 & 3.695567 & -0.516996 & 12 & -0.153797 & -0.155065 & -0.124202 & -0.157753 \\
\hline-0.647427 & 4.886859 & -0.277844 & 12 & -0.106405 & -0.104082 & -0.089665 & -0.097204 \\
\hline 1.405904 & 1.220471 & 0.286378 & 12 & -0.362743 & -0.374965 & -0.213112 & -0.358810 \\
\hline-1.406252 & 1.220572 & -0.286268 & 12 & -0.297967 & -0.312125 & -0.165308 & -0.306579 \\
\hline-0.729336 & 0.000005 & 0.000017 & 12 & 0.191790 & 0.201148 & 0.106027 & 0.190387 \\
\hline 0.728903 & -0.000005 & 0.000018 & 12 & 0.242394 & 0.250561 & 0.149624 & 0.238935 \\
\hline 1.405887 & -1.220489 & -0.286351 & 12 & -0.362738 & -0.374959 & -0.213113 & -0.358806 \\
\hline 0.686611 & -2.448104 & -0.218279 & 12 & 0.232196 & 0.234999 & 0.160739 & 0.224207 \\
\hline-0.686966 & -2.448141 & & 12 & 0.153073 & 0.157457 & 0.094709 & 0.157122 \\
\hline-1.406270 & -1.220554 & 0.286288 & 12 & -0.297979 & -0.312137 & -0.165307 & -0.306588 \\
\hline 1.139649 & 5.825094 & 0.504312 & 1 & 0.146823 & 0.145392 & 0.135627 & 0.143358 \\
\hline 2.299937 & 3.708473 & 0.923853 & 1 & 0.140596 & 0.139362 & 0.132777 & 0.137096 \\
\hline-2.300112 & 3.708648 & -0.923523 & 1 & 0.123011 & 0.122286 & 0.119944 & 0.122467 \\
\hline-1.139712 & 5.825185 & -0.503853 & 1 & 0.150419 & 0.148889 & 0.138152 & 0.146133 \\
\hline 1.299254 & -3.695479 & -0.517378 & 12 & -0.220918 & -0.220429 & -0.174596 & -0.211826 \\
\hline 0.647223 & -4.886813 & -0.278314 & 12 & -0.067873 & -0.066738 & -0.062104 & -0.067219 \\
\hline 2.299880 & -3.708497 & -0.923866 & 1 & 0.140595 & 0.139361 & 0.132779 & 0.137096 \\
\hline-1.299562 & -3.695554 & 0.516975 & 12 & -0.153809 & -0.155077 & -0.124196 & -0.157760 \\
\hline-0.647506 & -4.886853 & 0.277805 & 12 & -0.106395 & -0.104072 & -0.089671 & -0.097198 \\
\hline 1.139556 & -5.825105 & -0.504363 & 1 & 0.146824 & 0.145394 & 0.135627 & 0.143358 \\
\hline-2.300173 & -3.708625 & 0.923500 & 1 & 0.123012 & 0.122288 & 0.119942 & 0.122468 \\
\hline-1.139807 & -5.825175 & 0.503797 & 1 & 0.150418 & 0.148888 & 0.138153 & 0.146133 \\
\hline-2.798954 & 1.228850 & -0.813147 & 12 & 0.279529 & 0.295478 & 0.175736 & 0.313838 \\
\hline-3.042855 & 0.629923 & -2.059282 & 12 & -0.214176 & -0.220623 & -0.155433 & -0.230805 \\
\hline-3.850976 & 1.891814 & -0.164330 & 12 & -0.067880 & -0.072904 & -0.086026 & -0.092316 \\
\hline-4.305683 & 0.699034 & -2.644391 & 12 & -0.150968 & -0.145732 & -0.133186 & -0.135391 \\
\hline-2.234496 & 0.130226 & -2.581071 & 1 & 0.143091 & 0.142830 & 0.122061 & 0.142293 \\
\hline-5.117813 & 1.939958 & -0.740340 & 12 & -0.153852 & -0.150247 & -0.127263 & -0.138543 \\
\hline-3.682663 & 2.349347 & 0.803377 & 1 & 0.100325 & 0.099797 & 0.103555 & 0.101343 \\
\hline-5.347833 & 1.349884 & -1.984820 & 12 & -0.114177 & -0.113298 & -0.098290 & -0.116045 \\
\hline-4.472637 & 0.247168 & -3.615252 & 1 & 0.153966 & 0.151601 & 0.138435 & 0.148071 \\
\hline-5.924827 & 2.440397 & -0.218099 & 1 & 0.138284 & 0.136201 & 0.129159 & 0.133646 \\
\hline
\end{tabular}




\begin{tabular}{|c|c|c|c|c|c|c|c|}
\hline-6.331464 & 1.399716 & -2.436741 & 1 & 0.147393 & 0.145246 & 0.134165 & 0.143295 \\
\hline-2.798977 & -1.228820 & 0.813153 & 12 & 0.279572 & 0.295520 & 0.175722 & 0.313873 \\
\hline-3.851009 & -1.891728 & 0.164295 & 12 & -0.067898 & -0.072923 & -0.086016 & -0.092328 \\
\hline-3.042875 & -0.629949 & 2.059316 & 12 & -0.214190 & -0.220638 & -0.155429 & -0.230819 \\
\hline-5.117853 & -1.939868 & 0.740292 & 12 & -0.153842 & -0.150235 & -0.127269 & -0.138534 \\
\hline-3.682699 & -2.349218 & -0.803433 & 1 & 0.100327 & 0.099799 & 0.103554 & 0.101343 \\
\hline-4.305709 & -0.699058 & 2.644411 & 12 & -0.150968 & -0.145732 & -0.133185 & -0.135391 \\
\hline-2.234507 & -0.130299 & 2.581137 & 1 & 0.143092 & 0.142831 & 0.122062 & 0.142294 \\
\hline-5.347870 & -1.349849 & 1.984798 & 12 & -0.114187 & -0.113309 & -0.098284 & -0.116055 \\
\hline-5.924875 & -2.440261 & 0.218019 & 1 & 0.138281 & 0.136198 & 0.129161 & 0.133643 \\
\hline-4.472659 & -0.247237 & 3.615294 & 1 & 0.153968 & 0.151603 & 0.138434 & 0.148073 \\
\hline-6.331505 & -1.399679 & 2.436708 & 1 & 0.147395 & 0.145248 & 0.134164 & 0.143298 \\
\hline 2.798839 & 1.228505 & 0.812638 & 12 & 0.342739 & 0.354761 & 0.207807 & 0.353242 \\
\hline 3.850665 & 1.891382 & 0.163412 & 12 & -0.095155 & -0.097141 & -0.095374 & -0.108749 \\
\hline 3.043288 & 0.629150 & 2.058460 & 12 & -0.247630 & -0.250896 & -0.173162 & -0.248633 \\
\hline 5.117816 & 1.939119 & 0.738764 & 12 & -0.163751 & -0.160373 & -0.137160 & -0.148370 \\
\hline 3.681944 & 2.349154 & -0.804112 & 1 & 0.108787 & 0.107380 & 0.107357 & 0.107244 \\
\hline 4.306443 & 0.697843 & 2.642912 & 12 & -0.144653 & -0.140419 & -0.133437 & -0.132734 \\
\hline 2.235115 & 0.129433 & 2.580519 & 1 & 0.149350 & 0.148286 & 0.126039 & 0.145139 \\
\hline 5.348373 & 1.348669 & 1.982967 & 12 & -0.103810 & -0.103773 & -0.087619 & -0.107652 \\
\hline 5.924662 & 2.439508 & 0.216216 & 1 & 0.141887 & 0.139739 & 0.131584 & 0.136961 \\
\hline 4.473828 & 0.245657 & 3.613550 & 1 & 0.152903 & 0.150683 & 0.138767 & 0.147254 \\
\hline 6.332256 & 1.398169 & 2.434373 & 1 & 0.144295 & 0.142574 & 0.131499 & 0.141261 \\
\hline 3.681911 & -2.349255 & 0.804091 & 1 & 0.108785 & 0.107379 & 0.107358 & 0.107245 \\
\hline 3.850636 & -1.891453 & -0.163417 & 12 & -0.095140 & -0.097127 & -0.095381 & -0.108739 \\
\hline 5.117784 & -1.939193 & -0.738776 & 12 & -0.163758 & -0.160380 & -0.137156 & -0.148375 \\
\hline 2.798820 & -1.228535 & -0.812618 & 12 & 0.342707 & 0.354728 & 0.207816 & 0.353215 \\
\hline 5.348348 & -1.348704 & -1.982959 & 12 & -0.103805 & -0.103767 & -0.087622 & -0.107647 \\
\hline 5.924623 & -2.439616 & -0.216248 & 1 & 0.141889 & 0.139741 & 0.131583 & 0.136962 \\
\hline 3.043276 & -0.629139 & -2.058418 & 12 & -0.247619 & -0.250884 & -0.173166 & -0.248623 \\
\hline 4.306428 & -0.697834 & -2.642877 & 12 & -0.144650 & -0.140417 & -0.133438 & -0.132731 \\
\hline 6.332229 & -1.398206 & -2.434371 & 1 & 0.144293 & 0.142572 & 0.131500 & 0.141260 \\
\hline 2.235111 & -0.129387 & -2.580457 & 1 & 0.149348 & 0.148284 & 0.126039 & 0.145137 \\
\hline 4.473818 & -0.245616 & -3.613499 & 1 & 0.152901 & 0.150680 & 0.138768 & 0.147251 \\
\hline
\end{tabular}




\section{Appendix 4.1}

Optimized structures for metal OEP.

$\begin{array}{lrrr}\text { Structure of Radical Octaethylporphyrin (OEP) } \\ \text { C } & -1.62092000 & -2.40638400 & -0.93773500 \\ \mathrm{C} & -1.79886500 & -1.88635200 & -2.22997600 \\ \mathrm{C} & -2.17858000 & -3.71274300 & -0.50830600 \\ \mathrm{C} & -2.95399000 & -4.65644700 & -1.38119100 \\ \mathrm{C} & -2.06407500 & -5.50955100 & -2.30886900 \\ \mathrm{C} & -1.81352300 & -3.85590000 & 0.79252600 \\ \mathrm{C} & -2.08218500 & -4.99810900 & 1.72903700 \\ \mathrm{C} & -0.89770300 & -5.97755700 & 1.86222600 \\ \mathrm{C} & -1.03828500 & -2.63520600 & 1.12625500 \\ \mathrm{C} & -0.26423300 & 1.30565900 & -2.79481100 \\ \mathrm{C} & -0.85011400 & 1.06977400 & -4.11208200 \\ \mathrm{C} & -0.75221700 & 2.03059600 & -5.26214100 \\ \mathrm{C} & -1.77082300 & 3.18678600 & -5.18501300 \\ \mathrm{C} & -1.49696400 & -0.13947500 & -4.06455500 \\ \mathrm{C} & -2.27827200 & -0.82303600 & -5.14972700 \\ \mathrm{C} & -3.80139500 & -0.60409100 & -5.04100400 \\ \mathrm{C} & -1.32292400 & -0.67692000 & -2.71725100 \\ \mathrm{C} & -0.48452200 & -2.40432800 & 2.39591000 \\ \mathrm{H} & -3.67618400 & -4.09376100 & -1.98741700 \\ \mathrm{H} & -2.68089800 & -6.16782100 & -2.92928100 \\ \mathrm{H} & -1.45800500 & -4.88508600 & -2.97441000 \\ \mathrm{H} & -1.14550900 & -6.78344000 & 2.56064600 \\ \mathrm{H} & -0.65230900 & -6.42827600 & 0.89510400 \\ \mathrm{H} & 0.00315800 & -5.47416400 & 2.23029900 \\ \mathrm{H} & -2.79794300 & 2.80781400 & -5.19483100 \\ \mathrm{H} & -1.64774000 & 3.85777800 & -6.04102700 \\ \mathrm{H} & -1.64200900 & 3.77519000 & -4.27022400 \\ \mathrm{H} & -4.31860400 & -1.13425200 & -5.84697900 \\ \mathrm{H} & -4.05256600 & 0.45879000 & -5.11750100 \\ \mathrm{H} & -3.55123100 & -5.32288100 & -0.74911400 \\ \mathrm{H} & -4.19322200 & -0.97052900 & -4.08612600 \\ \mathrm{H} & -0.29961400 & 0.11735900 & -1.03940700 \\ \mathrm{H} & -2.96273600 & -5.54939200 & 1.38092000 \\ \mathrm{H} & -2.34670700 & -4.61033800 & 2.72152200 \\ \mathrm{H} & -0.89860500 & 1.48486900 & -6.20022600 \\ \mathrm{H} & 0.26351700 & 2.44280200 & -5.30963500 \\ \mathrm{H} & -1.93168000 & -0.46233900 & -6.12395000 \\ \mathrm{H} & -2.06281500 & -1.89872900 & -5.13363600 \\ \mathrm{H} & -0.65212000 & -3.16394900 & 3.15009700 \\ \mathrm{H} & -2.36817600 & -2.48843600 & -2.92800900 \\ \mathrm{H} & -1.37853700 & -6.13431200 & -1.72703600 \\ \mathrm{~N} & -0.94200500 & -1.78810300 & 0.06613700 \\ \mathrm{~N} & -0.58343800 & 0.23143600 & -2.00748800 \\ \mathrm{H} & 0.48452200 & 2.40432800 & -2.39591000 \\ & 0.26423300 & -1.30565900 & 2.79481100 \\ & 1.03828500 & 2.63520600 & -1.12625500 \\ & 0.65212000 & 3.16394900 & -3.15009700\end{array}$




\begin{tabular}{lrrr}
$\mathrm{C}$ & 0.85011400 & -1.06977400 & 4.11208200 \\
$\mathrm{~N}$ & 0.58343800 & -0.23143600 & 2.00748800 \\
$\mathrm{C}$ & 1.81352300 & 3.85590000 & -0.79252600 \\
$\mathrm{~N}$ & 0.94200500 & 1.78810300 & -0.06613700 \\
$\mathrm{C}$ & 0.75221700 & -2.03059600 & 5.26214100 \\
$\mathrm{C}$ & 1.49696400 & 0.13947500 & 4.06455500 \\
$\mathrm{C}$ & 1.32292400 & 0.67692000 & 2.71725100 \\
$\mathrm{H}$ & 0.29961400 & -0.11735900 & 1.03940700 \\
$\mathrm{C}$ & 2.17858000 & 3.71274300 & 0.50830600 \\
$\mathrm{C}$ & 2.08218500 & 4.99810900 & -1.72903700 \\
$\mathrm{C}$ & 1.62092000 & 2.40638400 & 0.93773500 \\
$\mathrm{C}$ & 1.77082300 & -3.18678600 & 5.18501300 \\
$\mathrm{H}$ & 0.89860500 & -1.48486900 & 6.20022600 \\
$\mathrm{H}$ & -0.26351700 & -2.44280200 & 5.30963500 \\
$\mathrm{C}$ & 2.27827200 & 0.82303600 & 5.14972700 \\
$\mathrm{C}$ & 1.79886500 & 1.88635200 & 2.22997600 \\
$\mathrm{C}$ & 2.95399000 & 4.65644700 & 1.38119100 \\
$\mathrm{C}$ & 0.89770300 & 5.97755700 & -1.86222600 \\
$\mathrm{H}$ & 2.96273600 & 5.54939200 & -1.38092000 \\
$\mathrm{H}$ & 2.34670700 & 4.61033800 & -2.72152200 \\
$\mathrm{H}$ & 2.79794300 & -2.80781400 & 5.19483100 \\
$\mathrm{H}$ & 1.64774000 & -3.85777800 & 6.04102700 \\
$\mathrm{H}$ & 1.64200900 & -3.77519000 & 4.27022400 \\
$\mathrm{C}$ & 3.80139500 & 0.60409100 & 5.04100400 \\
$\mathrm{H}$ & 1.93168000 & 0.46233900 & 6.12395000 \\
$\mathrm{H}$ & 2.06281500 & 1.89872900 & 5.13363600 \\
$\mathrm{H}$ & 2.36817600 & 2.48843600 & 2.92800900 \\
$\mathrm{C}$ & 2.06407500 & 5.50955100 & 2.30886900 \\
$\mathrm{H}$ & 3.67618400 & 4.09376100 & 1.98741700 \\
$\mathrm{H}$ & 3.55123100 & 5.32288100 & 0.74911400 \\
$\mathrm{H}$ & 1.14550900 & 6.78344000 & -2.56064600 \\
$\mathrm{H}$ & 0.65230900 & 6.42827600 & -0.89510400 \\
$\mathrm{H}$ & -0.00315800 & 5.47416400 & -2.23029900 \\
$\mathrm{H}$ & 4.31860400 & 1.13425200 & 5.84697900 \\
$\mathrm{H}$ & 4.05256600 & -0.45879000 & 5.11750100 \\
$\mathrm{H}$ & 4.19322200 & 0.97052900 & 4.08612600 \\
$\mathrm{H}$ & 2.68089800 & 6.16782100 & 2.92928100 \\
$\mathrm{H}$ & 1.45800500 & 4.88508600 & 2.97441000 \\
$\mathrm{H}$ & 1.37853700 & 6.13431200 & 1.72703600 \\
& & & \\
\hline
\end{tabular}


Structure of radical manganese Octaethylporphyrin (OEP)

\begin{tabular}{|c|c|c|c|}
\hline Mn & 0.00061400 & -0.00038300 & 0.00008300 \\
\hline $\mathrm{N}$ & 1.94191700 & -0.16154300 & 0.24873200 \\
\hline $\mathrm{N}$ & -0.16224400 & -1.95526100 & 0.00154600 \\
\hline $\mathrm{N}$ & -1.94026700 & 0.16076400 & -0.25179600 \\
\hline $\mathrm{N}$ & 0.16318400 & 1.95447500 & 0.00214100 \\
\hline $\mathrm{C}$ & 2.88532200 & 0.86207300 & 0.36169900 \\
\hline $\mathrm{C}$ & 2.70367800 & -1.32682700 & 0.35999800 \\
\hline $\mathrm{C}$ & 0.85127500 & -2.90559800 & 0.15146200 \\
\hline $\mathrm{C}$ & -1.31891200 & -2.72575100 & -0.14570400 \\
\hline $\mathrm{C}$ & -2.88365000 & -0.86283800 & -0.36509900 \\
\hline $\mathrm{C}$ & -2.70144000 & 1.32607200 & -0.36738900 \\
\hline $\mathrm{C}$ & -0.85035100 & 2.90492400 & -0.14727200 \\
\hline $\mathrm{C}$ & 1.31932400 & 2.72491000 & 0.15351800 \\
\hline $\mathrm{C}$ & 4.21138800 & 0.34300600 & 0.53287300 \\
\hline $\mathrm{C}$ & 4.09708500 & -1.03395600 & 0.53228500 \\
\hline $\mathrm{C}$ & 0.33363600 & -4.24121600 & 0.10330900 \\
\hline $\mathrm{C}$ & -1.02936100 & -4.12828400 & -0.09027400 \\
\hline $\mathrm{C}$ & -4.20887900 & -0.34382400 & -0.54276000 \\
\hline $\mathrm{C}$ & -4.09417400 & 1.03315500 & -0.54507200 \\
\hline $\mathrm{C}$ & -0.33341100 & 4.24052300 & -0.09268500 \\
\hline $\mathrm{C}$ & 1.02914000 & 4.12744000 & 0.10368800 \\
\hline $\mathrm{C}$ & 2.58827300 & 2.20949900 & 0.32518800 \\
\hline $\mathrm{H}$ & 3.40665200 & 2.90958100 & 0.43496900 \\
\hline $\mathrm{C}$ & -2.58740400 & -2.21041300 & -0.32157700 \\
\hline $\mathrm{H}$ & -3.40590900 & -2.91060600 & -0.42984700 \\
\hline $\mathrm{C}$ & -2.18631300 & 2.60601900 & -0.32473200 \\
\hline $\mathrm{H}$ & -2.87695400 & 3.43231100 & -0.43495200 \\
\hline $\mathrm{C}$ & 5.46205100 & 1.16601100 & 0.65496700 \\
\hline $\mathrm{H}$ & 6.22507500 & 0.58309600 & 1.18220900 \\
\hline $\mathrm{H}$ & 5.26927200 & 2.04361900 & 1.28383300 \\
\hline $\mathrm{C}$ & 5.19463200 & -2.05185900 & 0.65622900 \\
\hline $\mathrm{H}$ & 4.85470600 & -2.89040000 & 1.27598800 \\
\hline $\mathrm{H}$ & 6.03860900 & -1.60602100 & 1.19387100 \\
\hline $\mathrm{C}$ & 1.14179900 & -5.50438100 & 0.19714600 \\
\hline $\mathrm{H}$ & 0.51191500 & -6.30335400 & 0.60315000 \\
\hline $\mathrm{H}$ & 1.95824400 & -5.37361800 & 0.91710300 \\
\hline $\mathrm{C}$ & -2.03549200 & -5.24073700 & -0.17831400 \\
\hline $\mathrm{H}$ & -1.54142900 & -6.14207900 & -0.55683100 \\
\hline $\mathrm{H}$ & -2.80607200 & -4.99042500 & -0.91719500 \\
\hline $\mathrm{C}$ & -5.45936900 & -1.16669600 & -0.66775900 \\
\hline $\mathrm{H}$ & -5.26409200 & -2.04658300 & -1.29262700 \\
\hline $\mathrm{H}$ & -6.21962000 & -0.58525100 & -1.20063700 \\
\hline $\mathrm{C}$ & -5.19084000 & 2.05107400 & -0.67652800 \\
\hline $\mathrm{H}$ & -6.03176000 & 1.60463700 & -1.21848000 \\
\hline $\mathrm{H}$ & -4.84718200 & 2.88868200 & -1.29547200 \\
\hline $\mathrm{C}$ & -1.14159800 & 5.50383100 & -0.18385600 \\
\hline $\mathrm{H}$ & -1.95504200 & 5.37625400 & -0.90778900 \\
\hline $\mathrm{H}$ & -0.51025500 & 6.30487500 & -0.58347500 \\
\hline $\mathrm{C}$ & 2.03414100 & 5.24032300 & 0.19869400 \\
\hline $\mathrm{H}$ & 2.80614900 & 4.98526900 & 0.93439600 \\
\hline $\mathrm{H}$ & 1.53955000 & 6.13811500 & 0.58501000 \\
\hline $\mathrm{C}$ & 6.02926900 & 1.62560700 & -0.70436700 \\
\hline $\mathrm{H}$ & 6.93890200 & 2.21618100 & -0.55558000 \\
\hline
\end{tabular}




$\begin{array}{lrrr}\mathrm{H} & 5.30747400 & 2.24201800 & -1.25091200 \\ \mathrm{H} & 6.28071300 & 0.76851000 & -1.33739200 \\ \mathrm{C} & 5.69057400 & -2.58877800 & -0.70263000 \\ \mathrm{H} & 6.48609000 & -3.32553900 & -0.55190400 \\ \mathrm{H} & 6.08984000 & -1.78119900 & -1.32476800 \\ \mathrm{H} & 4.88106200 & -3.06978900 & -1.26203000 \\ \mathrm{C} & 1.72031200 & -5.95549400 & -1.16015600 \\ \mathrm{H} & 2.29622300 & -6.87866700 & -1.03997800 \\ \mathrm{H} & 2.38176200 & -5.19269300 & -1.58467900 \\ \mathrm{H} & 0.92254500 & -6.14430300 & -1.88604200 \\ \mathrm{C} & -2.70542800 & -5.56109800 & 1.17426800 \\ \mathrm{H} & -3.42367700 & -6.37906800 & 1.05832000 \\ \mathrm{H} & -3.23963900 & -4.69102900 & 1.57081200 \\ \mathrm{H} & -1.96331100 & -5.86344800 & 1.92048600 \\ \mathrm{C} & -6.03332500 & -1.62131200 & 0.69042800 \\ \mathrm{H} & -6.94237900 & -2.21218500 & 0.53928300 \\ \mathrm{H} & -6.28765400 & -0.76193500 & 1.31916200 \\ \mathrm{H} & -5.31444100 & -2.23598700 & 1.24274500 \\ \mathrm{C} & -5.69469200 & 2.59008300 & 0.67856500 \\ \mathrm{H} & -6.48903100 & 3.32688700 & 0.52196300 \\ \mathrm{H} & -4.88842500 & 3.07166600 & 1.24214400 \\ \mathrm{H} & -6.09800500 & 1.78361900 & 1.29952700 \\ \mathrm{C} & -1.72577600 & 5.94852200 & 1.17313100 \\ \mathrm{H} & -2.30113000 & 6.87228800 & 1.05487100 \\ \mathrm{H} & -0.93100000 & 6.13389500 & 1.90317500 \\ \mathrm{H} & -2.38899800 & 5.18380600 & 1.59136100 \\ \mathrm{C} & 2.70139000 & 5.57218800 & -1.15242400 \\ \mathrm{H} & 3.41932400 & 6.38964400 & -1.03102900 \\ \mathrm{H} & 1.95774300 & 5.88024500 & -1.89480000 \\ \mathrm{H} & 3.23541400 & 4.70578500 & -1.55716300 \\ \mathrm{C} & 2.18815400 & -2.60674600 & 0.32158100 \\ \mathrm{H} & 2.87908500 & -3.43298000 & 0.43016500 \\ & & & \end{array}$


Structure of bridge protonated Octaethylporphyrin (OEP)

\begin{tabular}{|c|c|c|c|}
\hline $\mathrm{C}$ & 0.68524000 & -2.97188500 & 0.17042300 \\
\hline $\mathrm{C}$ & -0.67063200 & -3.33336300 & 0.02184700 \\
\hline $\mathrm{C}$ & 1.76672000 & -3.94698700 & 0.33131100 \\
\hline $\mathrm{C}$ & 1.61453800 & -5.44275700 & 0.32373000 \\
\hline $\mathrm{C}$ & 1.52310600 & -6.05018000 & -1.09071800 \\
\hline $\mathrm{C}$ & 2.91196700 & -3.20569600 & 0.45711800 \\
\hline $\mathrm{C}$ & 4.32646600 & -3.68648000 & 0.62125100 \\
\hline $\mathrm{C}$ & 5.12917000 & -3.71016600 & -0.69528200 \\
\hline $\mathrm{C}$ & 2.48618200 & -1.80747900 & 0.36786600 \\
\hline $\mathrm{C}$ & -3.03268600 & -0.69948200 & -0.41440400 \\
\hline $\mathrm{C}$ & -3.92466400 & -1.81331000 & -0.46831900 \\
\hline $\mathrm{C}$ & -5.41558600 & -1.71662400 & -0.65558200 \\
\hline $\mathrm{C}$ & -6.20126500 & -1.54034800 & 0.65886100 \\
\hline $\mathrm{C}$ & -3.15167400 & -2.95780000 & -0.30225200 \\
\hline $\mathrm{C}$ & -3.62123100 & -4.38373400 & -0.25638000 \\
\hline $\mathrm{C}$ & -3.79853500 & -4.92794000 & 1.17678000 \\
\hline $\mathrm{C}$ & -1.78372800 & -2.53415300 & -0.14957800 \\
\hline $\mathrm{C}$ & 3.35747400 & -0.71566800 & 0.46006600 \\
\hline $\mathrm{H}$ & 0.72538200 & -5.73155600 & 0.89998500 \\
\hline $\mathrm{H}$ & 1.40911500 & -7.13786300 & -1.03494700 \\
\hline $\mathrm{H}$ & 0.66944000 & -5.64756400 & -1.64775600 \\
\hline $\mathrm{H}$ & 6.14869700 & -4.06765100 & -0.51690800 \\
\hline $\mathrm{H}$ & 4.65964900 & -4.37326700 & -1.42923900 \\
\hline $\mathrm{H}$ & 5.19285500 & -2.71305700 & -1.14550000 \\
\hline $\mathrm{H}$ & -6.03752300 & -2.38689200 & 1.33335000 \\
\hline $\mathrm{H}$ & -7.27486700 & -1.47096300 & 0.45620800 \\
\hline $\mathrm{H}$ & -5.90035100 & -0.63148100 & 1.19252500 \\
\hline $\mathrm{H}$ & -4.14570300 & -5.96557800 & 1.14659100 \\
\hline $\mathrm{H}$ & -4.53343500 & -4.33975400 & 1.73552200 \\
\hline $\mathrm{H}$ & 2.46343800 & -5.89358800 & 0.85011800 \\
\hline $\mathrm{H}$ & -2.85639000 & -4.89920100 & 1.73433900 \\
\hline $\mathrm{H}$ & -0.96207800 & -0.54709500 & -0.15300700 \\
\hline $\mathrm{H}$ & 4.31615000 & -4.69502300 & 1.04968800 \\
\hline $\mathrm{H}$ & 4.85136600 & -3.05988300 & 1.35430500 \\
\hline $\mathrm{H}$ & -5.77219900 & -2.61810000 & -1.16632100 \\
\hline $\mathrm{H}$ & -5.64927300 & -0.88320800 & -1.33022800 \\
\hline $\mathrm{H}$ & -4.57365100 & -4.46441800 & -0.79121500 \\
\hline $\mathrm{H}$ & -2.91564500 & -5.02312900 & -0.80110900 \\
\hline $\mathrm{H}$ & 4.40716900 & -0.94550300 & 0.61278100 \\
\hline $\mathrm{H}$ & -0.87730200 & -4.39896300 & 0.04177200 \\
\hline $\mathrm{H}$ & 2.42611300 & -5.83248400 & -1.67049000 \\
\hline $\mathrm{N}$ & 1.13491900 & -1.69395100 & 0.19293300 \\
\hline $\mathrm{N}$ & -1.78210600 & -1.14391500 & -0.22648400 \\
\hline $\mathrm{C}$ & 3.04915800 & 0.64052400 & 0.37690700 \\
\hline $\mathrm{C}$ & -2.42884200 & 1.83845000 & -0.39449700 \\
\hline $\mathrm{C}$ & 3.94863800 & 1.75975600 & 0.45158500 \\
\hline $\mathrm{N}$ & 1.77967300 & 1.14037400 & 0.20595200 \\
\hline $\mathrm{C}$ & -2.85929900 & 3.24584500 & -0.45513300 \\
\hline $\mathrm{N}$ & -1.13480200 & 1.68907200 & -0.20828300 \\
\hline $\mathrm{C}$ & 5.43999000 & 1.65750100 & 0.60335900 \\
\hline $\mathrm{C}$ & 3.18057500 & 2.91310100 & 0.32360800 \\
\hline $\mathrm{C}$ & 1.81563200 & 2.49704300 & 0.17260500 \\
\hline $\mathrm{H}$ & 0.94819700 & 0.56278800 & 0.13140300 \\
\hline
\end{tabular}




$\begin{array}{lrrr}\mathrm{C} & -1.72040800 & 3.98073900 & -0.29033200 \\ \mathrm{C} & -4.27184700 & 3.72796700 & -0.63797300 \\ \mathrm{C} & -0.63438200 & 3.00406000 & -0.13784400 \\ \mathrm{C} & 6.17724500 & 1.41637100 & -0.73005900 \\ \mathrm{H} & 5.81869800 & 2.57723600 & 1.06207800 \\ \mathrm{H} & 5.68591600 & 0.85044600 & 1.30437700 \\ \mathrm{C} & 3.65516600 & 4.34074100 & 0.31081100 \\ \mathrm{C} & 0.68302100 & 3.34109800 & 0.02483700 \\ \mathrm{C} & -1.56744200 & 5.47439200 & -0.24239200 \\ \mathrm{C} & -5.07943500 & 3.81146200 & 0.67372300 \\ \mathrm{H} & -4.25448300 & 4.71966900 & -1.10418900 \\ \mathrm{H} & -4.80205000 & 3.07759300 & -1.34607600 \\ \mathrm{H} & 5.99125200 & 2.23090300 & -1.43748100 \\ \mathrm{H} & 7.25693900 & 1.35245900 & -0.56120900 \\ \mathrm{H} & 5.85002900 & 0.48413000 & -1.20305100 \\ \mathrm{C} & 3.83307300 & 4.92077000 & -1.10648200 \\ \mathrm{H} & 4.60756800 & 4.40636000 & 0.84790600 \\ \mathrm{H} & 2.95435100 & 4.96888100 & 0.87501700 \\ \mathrm{H} & 0.90900600 & 4.40211600 & 0.03991800 \\ \mathrm{C} & -1.48629800 & 6.04365000 & 1.18908800 \\ \mathrm{H} & -0.67192300 & 5.77417200 & -0.80202200 \\ \mathrm{H} & -2.41215800 & 5.93912500 & -0.76336300 \\ \mathrm{H} & -6.09599300 & 4.16636800 & 0.47511900 \\ \mathrm{H} & -4.60926800 & 4.50309000 & 1.38007300 \\ \mathrm{H} & -5.15559400 & 2.83696500 & 1.17031800 \\ \mathrm{H} & 4.17844800 & 5.95834400 & -1.05379900 \\ \mathrm{H} & 4.56915100 & 4.34681200 & -1.67885000 \\ \mathrm{H} & 2.89176700 & 4.90369800 & -1.66685100 \\ \mathrm{H} & -1.37011500 & 7.13193900 & 1.16012600 \\ \mathrm{H} & -0.63746800 & 5.62533200 & 1.74078600 \\ \mathrm{H} & -2.39451900 & 5.81417600 & 1.75593400 \\ \mathrm{C} & -3.41663900 & 0.72887200 & -0.57318400 \\ \mathrm{H} & -3.85462500 & 0.84054500 & -1.58025300 \\ \mathrm{H} & -4.27048000 & 0.92226100 & 0.09259900\end{array}$




\begin{tabular}{|c|c|c|c|}
\hline \multicolumn{4}{|c|}{ Structure of N-pyrrole protonated Octaethylporphyrin (OEP) } \\
\hline $\mathrm{C}$ & -0.44990400 & -3.04209300 & 0.01745500 \\
\hline $\mathrm{C}$ & 0.91744900 & -3.34410400 & 0.07509000 \\
\hline $\mathrm{C}$ & -1.49040000 & -4.07983600 & -0.05739800 \\
\hline $\mathrm{C}$ & -1.25468200 & -5.56239200 & 0.01135700 \\
\hline $\mathrm{C}$ & -1.05128200 & -6.09133800 & 1.44562900 \\
\hline $\mathrm{C}$ & -2.67336000 & -3.41146300 & -0.18343200 \\
\hline $\mathrm{C}$ & -4.06246900 & -3.97571100 & -0.28460400 \\
\hline $\mathrm{C}$ & -4.83958700 & -3.94345800 & 1.04696300 \\
\hline $\mathrm{C}$ & -2.32763900 & -1.98120700 & -0.18167300 \\
\hline $\mathrm{C}$ & 3.07994600 & -0.48228800 & 0.22713500 \\
\hline $\mathrm{C}$ & 4.07038000 & -1.53266200 & 0.18398800 \\
\hline $\mathrm{C}$ & 5.55574100 & -1.30031800 & 0.18207600 \\
\hline $\mathrm{C}$ & 6.12544300 & -0.93901200 & -1.20434300 \\
\hline $\mathrm{C}$ & 3.40083100 & -2.74267700 & 0.10761800 \\
\hline $\mathrm{C}$ & 3.98930400 & -4.12205500 & 0.00713100 \\
\hline $\mathrm{C}$ & 4.08171000 & -4.64809300 & -1.43948900 \\
\hline $\mathrm{C}$ & 1.98860100 & -2.45511900 & 0.10368800 \\
\hline $\mathrm{C}$ & -3.28187600 & -0.97106400 & -0.36684100 \\
\hline $\mathrm{H}$ & -0.38338300 & -5.83065200 & -0.60051800 \\
\hline $\mathrm{H}$ & -0.88522800 & -7.17366100 & 1.43449900 \\
\hline $\mathrm{H}$ & -0.18870800 & -5.62047000 & 1.93010500 \\
\hline $\mathrm{H}$ & -5.84271800 & -4.36264000 & 0.91616000 \\
\hline $\mathrm{H}$ & -4.32377500 & -4.52848100 & 1.81545800 \\
\hline $\mathrm{H}$ & -4.94519600 & -2.92083300 & 1.42599200 \\
\hline $\mathrm{H}$ & 5.93911200 & -1.73894000 & -1.92821800 \\
\hline $\mathrm{H}$ & 7.20725000 & -0.78302900 & -1.14222200 \\
\hline $\mathrm{H}$ & 5.67162900 & -0.02260500 & -1.59713400 \\
\hline $\mathrm{H}$ & 4.50785200 & -5.65638600 & -1.45060200 \\
\hline $\mathrm{H}$ & 4.71870400 & -4.00372000 & -2.05418300 \\
\hline $\mathrm{H}$ & -2.10503200 & -6.08328500 & -0.44267400 \\
\hline $\mathrm{H}$ & 3.09482600 & -4.68994100 & -1.91262500 \\
\hline $\mathrm{H}$ & 0.93367600 & -0.68736800 & 0.32633000 \\
\hline $\mathrm{H}$ & -4.00600300 & -5.01097600 & -0.63902800 \\
\hline $\mathrm{H}$ & -4.63169500 & -3.43215900 & -1.05009500 \\
\hline $\mathrm{H}$ & 6.05871700 & -2.19939700 & 0.55378400 \\
\hline $\mathrm{H}$ & 5.80582100 & -0.50460700 & 0.89533600 \\
\hline $\mathrm{H}$ & 4.98871900 & -4.12163400 & 0.45536100 \\
\hline $\mathrm{H}$ & 3.39230300 & -4.81891400 & 0.60830200 \\
\hline $\mathrm{H}$ & -4.30759500 & -1.29336800 & -0.50680600 \\
\hline $\mathrm{H}$ & 1.18171300 & -4.39561000 & 0.07616800 \\
\hline $\mathrm{H}$ & -1.93023100 & -5.88883900 & 2.06647200 \\
\hline $\mathrm{N}$ & -0.98265800 & -1.78461400 & -0.04014700 \\
\hline $\mathrm{N}$ & 1.84613100 & -1.08960900 & 0.14890500 \\
\hline $\mathrm{C}$ & 3.31739600 & 0.88752600 & 0.34646200 \\
\hline $\mathrm{C}$ & -3.06964600 & 0.40380600 & -0.41741700 \\
\hline $\mathrm{C}$ & 2.39767100 & 1.93386800 & 0.32454600 \\
\hline $\mathrm{H}$ & 4.35164000 & 1.17193600 & 0.50143800 \\
\hline $\mathrm{C}$ & -4.02302600 & 1.45722100 & -0.66233000 \\
\hline $\mathrm{N}$ & -1.84321600 & 0.99327100 & -0.23329900 \\
\hline
\end{tabular}




$\begin{array}{lrrr}\mathrm{C} & 2.62889400 & 3.30877300 & 0.65805800 \\ \mathrm{~N} & 1.04458300 & 1.79882900 & 0.01870100 \\ \mathrm{C} & -5.48820200 & 1.23805400 & -0.91592000 \\ \mathrm{C} & -3.33853100 & 2.65891800 & -0.59763500 \\ \mathrm{C} & -1.95343600 & 2.36331700 & -0.31016200 \\ \mathrm{H} & -1.06263600 & 0.43549500 & 0.09127200 \\ \mathrm{C} & 1.42573600 & 3.99078300 & 0.51717900 \\ \mathrm{C} & 3.95377800 & 3.86746500 & 1.09839700 \\ \mathrm{C} & 0.43108300 & 3.04729400 & 0.09830700 \\ \mathrm{C} & -6.31740100 & 1.07952400 & 0.37460700 \\ \mathrm{H} & -5.88448300 & 2.07849400 & -1.49614500 \\ \mathrm{H} & -5.62186500 & 0.34955400 & -1.54491100 \\ \mathrm{C} & -3.89279300 & 4.04546800 & -0.77715100 \\ \mathrm{C} & -0.92293000 & 3.28656700 & -0.12915700 \\ \mathrm{C} & 1.16360800 & 5.44968900 & 0.77130000 \\ \mathrm{C} & 4.87063800 & 4.26432100 & -0.07675500 \\ \mathrm{H} & 3.78528600 & 4.74409300 & 1.73313300 \\ \mathrm{H} & 4.46836100 & 3.13468300 & 1.73114800 \\ \mathrm{H} & -6.24700100 & 1.97430200 & 1.00160300 \\ \mathrm{H} & -7.37231800 & 0.91760500 & 0.13103800 \\ \mathrm{H} & -5.97127100 & 0.22816700 & 0.97066500 \\ \mathrm{C} & -4.24079900 & 4.75053800 & 0.54960400 \\ \mathrm{H} & -4.79170900 & 3.99393800 & -1.40102300 \\ \mathrm{H} & -3.17643700 & 4.65812600 & -1.33821400 \\ \mathrm{H} & -1.22180700 & 4.32799600 & -0.13233000 \\ \mathrm{C} & 1.20324800 & 6.31039200 & -0.50813500 \\ \mathrm{H} & 0.18887200 & 5.56750400 & 1.25986800 \\ \mathrm{H} & 1.90303700 & 5.83215700 & 1.48300900 \\ \mathrm{H} & 5.81828700 & 4.66280800 & 0.29928900 \\ \mathrm{H} & 4.40331400 & 5.03296600 & -0.70073100 \\ \mathrm{H} & 5.09395700 & 3.40563100 & -0.71937600 \\ \mathrm{H} & -4.63392900 & 5.75359000 & 0.35514000 \\ \mathrm{H} & -4.99907700 & 4.19137500 & 1.10707200 \\ \mathrm{H} & -3.36159800 & 4.84950500 & 1.19572600 \\ \mathrm{H} & 1.00610100 & 7.35943800 & -0.26558100 \\ \mathrm{H} & 0.45323200 & 5.98314900 & -1.23648300 \\ \mathrm{H} & 2.18307700 & 6.25276500 & -0.99307000 \\ \mathrm{H} & 0.74338100 & 1.12528400 & -0.67639500 \\ & & & \end{array}$


Structure of bridge protonated zinc Octaethylporphyrin (OEP)

\begin{tabular}{|c|c|c|c|}
\hline C & 1.29367200 & -2.78139500 & -0.15110700 \\
\hline C & 0.00089500 & 3.42661000 & -0.00075800 \\
\hline C & -1.24187500 & 2.81477600 & 0.16211100 \\
\hline C & -2.49870300 & 3.51378200 & 0.32930000 \\
\hline $\mathrm{C}$ & -3.46690000 & 2.53774100 & 0.46198500 \\
\hline $\mathrm{C}$ & -2.77347100 & 1.27353800 & 0.37012000 \\
\hline $\mathrm{C}$ & -3.39698400 & 0.00491200 & 0.45729600 \\
\hline $\mathrm{C}$ & -2.81555400 & -1.23392100 & 0.3592160 \\
\hline $\mathrm{C}$ & -3.51183900 & -2.50693400 & 0.4531650 \\
\hline $\mathrm{C}$ & -2.55162600 & -3.48247000 & 0.3176180 \\
\hline $\mathrm{C}$ & -1.29455400 & -2.78097700 & 0.1492180 \\
\hline $\mathrm{C}$ & 2.55071500 & -3.48344900 & -0.3175010 \\
\hline C & 2.72740900 & -4.97808200 & -0.3189650 \\
\hline C & 2.86955600 & -5.59454500 & 1.08743700 \\
\hline $\mathrm{C}$ & 4.99203100 & -2.68116100 & -0.6334980 \\
\hline C & 5.79077000 & -2.58906000 & 0.68337200 \\
\hline $\mathrm{C}$ & 4.95114900 & 2.71607400 & -0.63838400 \\
\hline $\mathrm{C}$ & 5.74858500 & 2.63550600 & 0.67858200 \\
\hline C & 2.68103600 & 5.00467400 & -0.3267130 \\
\hline $\mathrm{C}$ & 2.76934900 & 5.61539800 & 1.08697200 \\
\hline $\mathrm{C}$ & -2.67840400 & 5.00600800 & 0.32757600 \\
\hline $\mathrm{C}$ & 3.51149800 & -2.50835700 & -0.45191300 \\
\hline $\mathrm{C}$ & -2.76748700 & 5.61725700 & -1.08583600 \\
\hline C & -4.94920200 & 2.71828900 & 0.64131300 \\
\hline $\mathrm{C}$ & -5.74826500 & 2.63828500 & -0.67470400 \\
\hline $\mathrm{C}$ & -4.99213100 & -2.67913700 & 0.63722700 \\
\hline $\mathrm{C}$ & -5.79299100 & -2.58688700 & -0.67834500 \\
\hline $\mathrm{C}$ & -2.72891700 & -4.97702500 & 0.31984300 \\
\hline $\mathrm{C}$ & -2.87318600 & -5.59390000 & -1.0861620 \\
\hline $\mathrm{C}$ & 2.81554200 & -1.23506700 & -0.3593510 \\
\hline $\mathrm{C}$ & 3.39763300 & 0.00343700 & -0.4566770 \\
\hline $\mathrm{C}$ & 2.77444800 & 1.27220400 & -0.3701500 \\
\hline $\mathrm{C}$ & 3.46854700 & 2.53611500 & -0.4609240 \\
\hline C & 2.50063900 & 3.51253400 & -0.32912500 \\
\hline C & 1.24333800 & 2.81403300 & -0.16336800 \\
\hline $\mathrm{H}$ & 4.47075500 & 0.01684800 & -0.61688500 \\
\hline $\mathrm{H}$ & 5.36909200 & -1.92962800 & -1.3381750 \\
\hline $\mathrm{H}$ & 5.18542400 & -3.65302400 & -1.10133800 \\
\hline $\mathrm{H}$ & 5.65108700 & -1.61769400 & 1.1700560 \\
\hline $\mathrm{H}$ & 5.47644100 & -3.36316300 & 1.3908560 \\
\hline $\mathrm{H}$ & 6.85984200 & -2.72134600 & 0.4883900 \\
\hline $\mathrm{H}$ & 5.33605800 & 1.96657200 & -1.3415690 \\
\hline $\mathrm{H}$ & 5.14029500 & 3.68644700 & -1.1111930 \\
\hline $\mathrm{H}$ & 5.61406700 & 1.66561700 & 1.17084600 \\
\hline $\mathrm{H}$ & 5.42646800 & 3.40994700 & 1.38241000 \\
\hline $\mathrm{H}$ & 6.81794400 & 2.77374700 & 0.4880740 \\
\hline $\mathrm{H}$ & 0.00122300 & 4.51157600 & -0.00074600 \\
\hline $\mathrm{H}$ & 3.59132700 & 5.25748400 & -0.88187600 \\
\hline $\mathrm{H}$ & 1.85759100 & 5.47990700 & -0.8746390 \\
\hline
\end{tabular}




\begin{tabular}{lrrr}
$\mathrm{H}$ & 1.86084900 & 5.41772700 & 1.66654300 \\
$\mathrm{H}$ & 3.61466400 & 5.19905800 & 1.64437500 \\
$\mathrm{H}$ & 2.90426800 & 6.70022600 & 1.02579000 \\
$\mathrm{H}$ & -1.85429700 & 5.48064300 & 0.87502800 \\
$\mathrm{H}$ & -3.58814300 & 5.25907000 & 0.88352500 \\
$\mathrm{H}$ & -3.61346900 & 5.20157900 & -1.64272500 \\
$\mathrm{H}$ & -1.85954400 & 5.41928100 & -1.66617800 \\
$\mathrm{H}$ & -2.90175600 & 6.70214100 & -1.02418800 \\
$\mathrm{H}$ & -4.46990100 & 0.01873000 & 0.61884200 \\
$\mathrm{H}$ & -5.13737800 & 3.68865200 & 1.11452600 \\
$\mathrm{H}$ & -5.33354700 & 1.96880400 & 1.34482500 \\
$\mathrm{H}$ & -5.61474100 & 1.66842800 & -1.16730400 \\
$\mathrm{H}$ & -5.42666800 & 3.41272300 & -1.37877300 \\
$\mathrm{H}$ & -6.81733600 & 2.77693500 & -0.48288400 \\
$\mathrm{H}$ & -5.18512800 & -3.65086600 & 1.10550100 \\
$\mathrm{H}$ & -5.36773000 & -1.92737000 & 1.34243200 \\
$\mathrm{H}$ & -5.48010600 & -3.36119400 & -1.38624600 \\
$\mathrm{H}$ & -5.65373700 & -1.61563200 & -1.16537400 \\
$\mathrm{H}$ & -6.86179100 & -2.71874600 & -0.48160100 \\
$\mathrm{H}$ & -1.88530500 & -5.45336000 & 0.83612000 \\
$\mathrm{H}$ & -3.61643000 & -5.23124800 & 0.91031400 \\
$\mathrm{H}$ & -3.74603800 & -5.18702400 & -1.60663400 \\
$\mathrm{H}$ & -1.99501200 & -5.39484900 & -1.71149100 \\
$\mathrm{H}$ & -2.99494400 & -6.67974000 & -1.01666600 \\
$\mathrm{H}$ & 1.88431700 & -5.45426500 & -0.83623700 \\
$\mathrm{H}$ & 3.61562100 & -5.23283800 & -0.90815300 \\
$\mathrm{H}$ & 1.99060300 & -5.39499700 & 1.71151300 \\
$\mathrm{H}$ & 3.74182600 & -5.18777200 & 1.60895500 \\
$\mathrm{H}$ & 2.99105700 & -6.68044500 & 1.01846000 \\
$\mathrm{~N}$ & 1.44245000 & -1.45739100 & -0.16964100 \\
$\mathrm{~N}$ & 1.43662900 & 1.45168900 & -0.19329300 \\
$\mathrm{~N}$ & -1.43574200 & 1.45258000 & 0.19207400 \\
$\mathrm{~N}$ & -1.44281700 & -1.45693700 & 0.16775300 \\
$\mathrm{Zn}$ & -0.00080900 & 0.01346700 & -0.00443200 \\
$\mathrm{C}$ & -0.00064200 & -3.49957100 & -0.00170800 \\
$\mathrm{H}$ & 0.09665200 & -4.18882300 & 0.85282300 \\
$\mathrm{H}$ & -0.09832100 & -4.18710400 & -0.85762100 \\
& & & \\
\hline
\end{tabular}


Structure of N-pyrrole protonated zinc Octaethylporphyrin (OEP)

\begin{tabular}{|c|c|c|c|}
\hline $\mathrm{C}$ & 2.88702100 & -1.24266900 & -0.00256100 \\
\hline C & -2.35117700 & 2.51064700 & -0.23834400 \\
\hline $\mathrm{C}$ & -2.91926900 & 1.26199400 & -0.03763100 \\
\hline $\mathrm{C}$ & -4.21690800 & 0.92164500 & 0.43256100 \\
\hline C & -4.27636700 & -0.46969600 & 0.57197100 \\
\hline C & -3.01706200 & -1.00516100 & 0.1870650 \\
\hline C & -2.56157300 & -2.31357200 & 0.2310310 \\
\hline $\mathrm{C}$ & -1.23931300 & -2.76221500 & 0.1420300 \\
\hline $\mathrm{C}$ & -0.87898400 & -4.16541900 & 0.1961900 \\
\hline C & 0.49274600 & -4.22226200 & 0.1565040 \\
\hline C & 0.96705600 & -2.85227100 & 0.0848540 \\
\hline $\mathrm{C}$ & 4.29751900 & -0.90245500 & -0.1153880 \\
\hline C & 2.32506700 & -2.51604400 & 0.0670510 \\
\hline $\mathrm{C}$ & 5.43064500 & -1.88799100 & -0.0706130 \\
\hline $\mathrm{C}$ & 5.82101700 & -2.30750700 & 1.3611470 \\
\hline C & 5.57172700 & 1.33941100 & $-0.3958510 c$ \\
\hline $\mathrm{C}$ & 6.02888300 & 1.97458800 & 0.9328320 \\
\hline C & 1.83667400 & 5.22937600 & -0.79098600 \\
\hline $\mathrm{C}$ & 2.41302800 & 5.75306600 & 0.54017100 \\
\hline C & -1.37834700 & 5.39574000 & -0.6984410 \\
\hline C & -1.79952800 & 5.98671700 & 0.6623370 \\
\hline $\mathrm{C}$ & -5.29610900 & 1.91801900 & 0.7519540 \\
\hline $\mathrm{C}$ & 4.35724100 & 0.46637800 & -0.25364900 \\
\hline $\mathrm{C}$ & -6.11448800 & 2.34310700 & -0.4846470 \\
\hline C & -5.43441500 & -1.28758400 & 1.07209400 \\
\hline C & -6.30875000 & -1.85818800 & -0.06349400 \\
\hline $\mathrm{C}$ & -1.85052900 & -5.31205500 & 0.24742700 \\
\hline C & -2.44634600 & -5.68104600 & -1.1258490 \\
\hline $\mathrm{C}$ & 1.36645200 & -5.44581800 & 0.1621450 \\
\hline $\mathrm{C}$ & 1.83211800 & -5.87693700 & -1.2434780 \\
\hline C & 2.98259200 & 0.94299800 & -0.2210640 \\
\hline $\mathrm{C}$ & 2.53398700 & 2.25034700 & -0.4007700 \\
\hline C & 1.21105500 & 2.70332200 & -0.4396310 \\
\hline C & 0.85880600 & 4.09943600 & -0.6243830 \\
\hline $\mathrm{C}$ & -0.51203300 & 4.17115000 & -0.5835460 \\
\hline C & -0.99398200 & 2.81877700 & -0.3823840 \\
\hline $\mathrm{H}$ & 3.29661900 & 3.00838900 & -0.5387080 \\
\hline $\mathrm{H}$ & 5.37112900 & 2.13361200 & -1.12629700 \\
\hline $\mathrm{H}$ & 6.39555500 & 0.75027200 & -0.8138410 \\
\hline $\mathrm{H}$ & 5.24154300 & 2.59882800 & 1.3696730 \\
\hline $\mathrm{H}$ & 6.28847300 & 1.20549300 & 1.6677550 \\
\hline $\mathrm{H}$ & 6.91102600 & 2.60302400 & 0.7725300 \\
\hline $\mathrm{H}$ & 2.65963300 & 4.91560400 & -1.4450390 \\
\hline $\mathrm{H}$ & 1.34180800 & 6.05553500 & -1.3134430 \\
\hline $\mathrm{H}$ & 2.94414600 & 4.96535500 & 1.0854480 \\
\hline $\mathrm{H}$ & 1.61839900 & 6.12915400 & 1.19284600 \\
\hline $\mathrm{H}$ & 3.11592400 & 6.57190300 & 0.3554370 \\
\hline $\mathrm{H}$ & -3.03042400 & 3.35506900 & -0.2168770 \\
\hline $\mathbf{H}$ & -0.84020200 & 6.16228500 & -1.266754 \\
\hline
\end{tabular}




$\begin{array}{lrrr}\mathrm{H} & -2.27370100 & 5.16524600 & -1.28894000 \\ \mathrm{H} & -2.36857200 & 5.26369400 & 1.25740400 \\ \mathrm{H} & -0.92453500 & 6.28138000 & 1.25062000 \\ \mathrm{H} & -2.42497800 & 6.87350600 & 0.51668000 \\ \mathrm{H} & -4.84706500 & 2.80578400 & 1.21291200 \\ \mathrm{H} & -5.97264300 & 1.49691100 & 1.50339200 \\ \mathrm{H} & -6.61720100 & 1.48489500 & -0.94224800 \\ \mathrm{H} & -5.47671500 & 2.80143000 & -1.24818000 \\ \mathrm{H} & -6.88012000 & 3.07134200 & -0.19924200 \\ \mathrm{H} & -3.31075300 & -3.07498100 & 0.41603900 \\ \mathrm{H} & -6.05743700 & -0.67418300 & 1.73189200 \\ \mathrm{H} & -5.05842300 & -2.11115400 & 1.69046200 \\ \mathrm{H} & -5.72889900 & -2.50956300 & -0.72630200 \\ \mathrm{H} & -6.73718100 & -1.05743800 & -0.67490100 \\ \mathrm{H} & -7.13378500 & -2.44480000 & 0.35272500 \\ \mathrm{H} & -1.34596400 & -6.19015900 & 0.66473700 \\ \mathrm{H} & -2.66508200 & -5.08178700 & 0.94618700 \\ \mathrm{H} & -1.65982500 & -5.96973500 & -1.83079800 \\ \mathrm{H} & -2.99293500 & -4.83939100 & -1.56533200 \\ \mathrm{H} & -3.14002600 & -6.52254500 & -1.02824500 \\ \mathrm{H} & 3.01729800 & -3.34989600 & 0.09411500 \\ \mathrm{H} & 2.24298200 & -5.27520300 & 0.79928300 \\ \mathrm{H} & 0.82104000 & -6.27436200 & 0.62674800 \\ \mathrm{H} & 0.97726700 & -6.11053900 & -1.88663800 \\ \mathrm{H} & 2.41023500 & -5.08667000 & -1.73455700 \\ \mathrm{H} & 2.46243900 & -6.76986200 & -1.17935900 \\ \mathrm{H} & 5.16485200 & -2.78183900 & -0.64944200 \\ \mathrm{H} & 6.30607800 & -1.45806700 & -0.56988900 \\ \mathrm{H} & 4.97713500 & -2.76717200 & 1.88733000 \\ \mathrm{H} & 6.14750000 & -1.44325300 & 1.94889700 \\ \mathrm{H} & 6.64187400 & -3.03167100 & 1.33690600 \\ \mathrm{~N} & 2.12947700 & -0.10836100 & -0.04951300 \\ \mathrm{~N} & 0.06681800 & 1.93113800 & -0.31307500 \\ \mathrm{~N} & -2.17098500 & 0.06810000 & -0.18852300 \\ \mathrm{~N} & -0.10445900 & -1.97316300 & 0.05427400 \\ \mathrm{Zn} & 0.19201000 & -0.00414500 & 0.14701500 \\ \mathrm{H} & -1.78349400 & -0.04106600 & -1.12748300 \\ & & & \end{array}$


Structure of bridge protonated nickel Octaethylporphyrin (OEP)

\begin{tabular}{|c|c|c|c|}
\hline $\mathrm{C}$ & -2.51131100 & -1.70991600 & -0.03528000 \\
\hline C & -1.77672000 & -2.89898400 & 0.16093800 \\
\hline $\mathrm{C}$ & -4.88767400 & -2.81396800 & -0.05308300 \\
\hline $\mathrm{C}$ & -5.23234500 & -3.13043700 & 1.4158530 \\
\hline $\mathrm{C}$ & -5.57178900 & 0.25637000 & -0.8542070 \\
\hline C & -6.27657800 & 0.84040400 & 0.3869520 \\
\hline $\mathrm{C}$ & -2.72727800 & 4.83912700 & -0.232065 \\
\hline $\mathrm{C}$ & -2.76307700 & 5.27560100 & -1.710756 \\
\hline $\mathrm{C}$ & 0.15425000 & 5.35890600 & 1.1707610 \\
\hline $\mathrm{C}$ & 1.00224800 & 6.11259600 & 0.1273390 \\
\hline C & -3.93895700 & -1.65795800 & -0.2159080 \\
\hline $\mathrm{C}$ & -4.23021700 & -0.35305700 & -0.558187 \\
\hline $\mathrm{C}$ & -2.97579400 & 0.35936100 & -0.5185260 \\
\hline $\mathrm{C}$ & -2.84409700 & 1.73848900 & -0.5900970 \\
\hline $\mathrm{C}$ & -1.70035600 & 2.42861300 & -0.2142430 \\
\hline C & -1.64406400 & 3.84245300 & 0.0700870 \\
\hline $\mathrm{C}$ & -0.41840700 & 4.06274200 & 0.6659550 \\
\hline C & -0.42574300 & -2.99788500 & 0.0061430 \\
\hline $\mathrm{H}$ & -3.72449400 & 2.31816600 & -0.8441850 \\
\hline $\mathrm{H}$ & -7.24540000 & 1.26853700 & 0.1096150 \\
\hline $\mathrm{H}$ & -6.45097900 & 0.06707800 & 1.1420300 \\
\hline $\mathrm{H}$ & -5.67606400 & 1.62910900 & 0.8533610 \\
\hline $\mathrm{H}$ & -3.70504800 & 4.42518800 & 0.0458510 \\
\hline $\mathrm{H}$ & -2.58846100 & 5.72292200 & 0.4001270 \\
\hline $\mathrm{H}$ & -1.81606100 & 5.73786700 & -2.0078690 \\
\hline $\mathrm{H}$ & -2.93803900 & 4.42266800 & -2.3756870 \\
\hline $\mathrm{H}$ & -3.56348900 & 6.00441300 & -1.8746880 \\
\hline $\mathrm{H}$ & -0.66577700 & 6.00726600 & 1.4998930 \\
\hline $\mathrm{H}$ & 0.75977900 & 5.17470700 & 2.0672840 \\
\hline $\mathrm{H}$ & 1.39125000 & 7.04484900 & 0.5499240 \\
\hline $\mathrm{H}$ & 0.40777900 & 6.36322000 & -0.7573520 \\
\hline $\mathrm{H}$ & 1.85422200 & 5.50973100 & -0.2075270 \\
\hline $\mathrm{H}$ & -5.81276900 & -2.59555700 & -0.597943 \\
\hline $\mathrm{H}$ & -4.33734300 & -3.38864800 & 1.993185 \\
\hline $\mathrm{H}$ & -5.92718000 & -3.97471400 & 1.4729730 \\
\hline $\mathrm{H}$ & -5.70186800 & -2.27034900 & 1.904377 \\
\hline $\mathrm{H}$ & -5.46384100 & 1.04028200 & -1.6139650 \\
\hline $\mathrm{H}$ & -6.21775000 & -0.50556600 & -1.3052130 \\
\hline $\mathrm{Ni}$ & -0.07413300 & -0.04128000 & -0.102323 \\
\hline $\mathrm{N}$ & -1.93171800 & -0.48883100 & -0.215412 \\
\hline $\mathrm{N}$ & -0.52191100 & 1.80415800 & 0.1363020 \\
\hline $\mathrm{C}$ & 0.25472900 & 2.78989600 & 0.6685400 \\
\hline $\mathrm{C}$ & 0.32890500 & -4.23100800 & -0.1235900 \\
\hline $\mathrm{N}$ & 0.39228200 & -1.89776600 & -0.302992 \\
\hline $\mathrm{C}$ & 1.60070300 & 2.60088400 & 1.0485520 \\
\hline $\mathrm{C}$ & -0.18777200 & -5.60304400 & 0.196179 \\
\hline C & 1.57187700 & -3.86984200 & -0.589310 \\
\hline $\mathrm{C}$ & 1.56067700 & -2.42892700 & $-0.663367 c$ \\
\hline $\mathrm{C}$ & 2.33563600 & 1.50093600 & 0.7155320 \\
\hline
\end{tabular}




$\begin{array}{lrrr}\mathrm{C} & 2.85140800 & -0.31987500 & -0.35100700 \\ \mathrm{H} & 2.10582400 & 3.42843300 & 1.53408700 \\ \mathrm{C} & -0.19785100 & -5.91172000 & 1.70782200 \\ \mathrm{H} & 0.42610900 & -6.34875500 & -0.32042300 \\ \mathrm{H} & -1.20358900 & -5.72148300 & -0.20215000 \\ \mathrm{C} & 2.74258300 & -4.75311500 & -0.92752100 \\ \mathrm{C} & 3.78131700 & 1.39541200 & 0.76679600 \\ \mathrm{C} & 4.10707700 & 0.26572900 & 0.05105500 \\ \mathrm{H} & -0.57932600 & -6.92217000 & 1.88604700 \\ \mathrm{H} & 0.81090000 & -5.84946400 & 2.12890700 \\ \mathrm{H} & -0.83188500 & -5.20623600 & 2.25548000 \\ \mathrm{C} & 3.72731500 & -4.95695600 & 0.24097500 \\ \mathrm{H} & 3.28132800 & -4.34211300 & -1.79083900 \\ \mathrm{H} & 2.37033200 & -5.73090900 & -1.25321100 \\ \mathrm{C} & 4.70219600 & 2.38663600 & 1.41817700 \\ \mathrm{C} & 5.47248900 & -0.27890400 & -0.26818600 \\ \mathrm{H} & 3.22953400 & -5.42477500 & 1.09667800 \\ \mathrm{H} & 4.14985900 & -4.00616200 & 0.58675600 \\ \mathrm{H} & 4.55686000 & -5.60293600 & -0.06451800 \\ \mathrm{C} & 5.16643200 & 3.51455000 & 0.47336200 \\ \mathrm{H} & 5.58277100 & 1.85659900 & 1.79944900 \\ \mathrm{H} & 4.21328500 & 2.82422800 & 2.29664200 \\ \mathrm{C} & 5.92558300 & -0.01805000 & -1.71783900 \\ \mathrm{H} & 5.50146600 & -1.35921400 & -0.06993600 \\ \mathrm{H} & 6.20054300 & 0.16510900 & 0.41946000 \\ \mathrm{H} & 4.31675100 & 4.08873600 & 0.08820000 \\ \mathrm{H} & 5.83069600 & 4.20408300 & 1.00409400 \\ \mathrm{H} & 5.71198300 & 3.11104100 & -0.38563600 \\ \mathrm{H} & 6.92684100 & -0.42767100 & -1.88605300 \\ \mathrm{H} & 5.95644800 & 1.05506900 & -1.93308900 \\ \mathrm{H} & 5.24845800 & -0.48126800 & -2.44467400 \\ \mathrm{H} & -4.47078900 & -3.71097100 & -0.52895500 \\ \mathrm{H} & -2.33764200 & -3.80945800 & 0.34004100 \\ \mathrm{~N} & 1.79524800 & 0.39349200 & 0.04225700 \\ \mathrm{C} & 2.71346700 & -1.59737900 & -1.09610700 \\ \mathrm{H} & 3.63882200 & -2.17260400 & -1.02743300 \\ \mathrm{H} & 2.59120900 & -1.36587900 & -2.16959500 \\ & & & \\ & & & \\ & & & \end{array}$


Structure of N-pyrrole protonated nickel Octaethylporphyrin (OEP)

$\begin{array}{lrrr}\mathrm{C} & 2.02876500 & 1.95413400 & 0.73542700 \\ \mathrm{C} & 3.01953200 & 1.00083400 & 0.88030800 \\ \mathrm{C} & 3.49368000 & 4.00618100 & 1.45773700 \\ \mathrm{C} & 4.56087400 & 4.28729500 & 0.38087800 \\ \mathrm{C} & 0.80964200 & 5.46365600 & 0.36885800 \\ \mathrm{C} & 0.99315400 & 6.04142000 & -1.04916700 \\ \mathrm{C} & -4.06368600 & 3.71191700 & -0.99762800 \\ \mathrm{C} & -4.39399000 & 4.50158400 & 0.28549300 \\ \mathrm{C} & -5.53028000 & 0.83626300 & -0.72242800 \\ \mathrm{C} & -6.23573300 & 0.73263400 & 0.64499600 \\ \mathrm{C} & 2.23833100 & 3.37691400 & 0.92045300 \\ \mathrm{C} & 1.10349300 & 3.99247500 & 0.46251200 \\ \mathrm{C} & 0.19959600 & 2.93135200 & 0.06310000 \\ \mathrm{C} & -1.09902300 & 3.13749600 & -0.36475000 \\ \mathrm{C} & -2.03313700 & 2.12775400 & -0.50539000 \\ \mathrm{C} & -3.44398400 & 2.36712800 & -0.73843500 \\ \mathrm{C} & -4.06294600 & 1.15095800 & -0.62197500 \\ \mathrm{C} & 2.88348400 & -0.31094500 & 0.46239200 \\ \mathrm{H} & -1.42894500 & 4.15801000 & -0.51287100 \\ \mathrm{H} & 0.76966500 & 7.11321900 & -1.05634000 \\ \mathrm{H} & 2.02195200 & 5.90645600 & -1.39866400 \\ \mathrm{H} & 0.33148400 & 5.55177200 & -1.77214200 \\ \mathrm{H} & -3.39859400 & 4.31017700 & -1.63235700 \\ \mathrm{H} & -4.98340600 & 3.57455200 & -1.57750600 \\ \mathrm{H} & -5.10837100 & 3.95654300 & 0.91093400 \\ \mathrm{H} & -3.49702500 & 4.68146900 & 0.88786900 \\ \mathrm{H} & -4.83499600 & 5.47140300 & 0.03309400 \\ \mathrm{H} & -6.02052900 & 1.61480800 & -1.31735500 \\ \mathrm{H} & -5.67589400 & -0.09765600 & -1.27951800 \\ \mathrm{H} & -7.29834500 & 0.50530600 & 0.51093000 \\ \mathrm{H} & -6.15689500 & 1.67306100 & 1.19983400 \\ \mathrm{H} & -5.79615800 & -0.05448800 & 1.26779600 \\ \mathrm{H} & 3.23383400 & 4.94784800 & 1.95482900 \\ \mathrm{H} & 5.187162900 & 3.36744500 & -0.12672400 \\ \mathrm{H} & 5.94700800 & -1.85321500 & 0.90486500 \\ \mathrm{H} & & & \end{array}$




$\begin{array}{lrrr}\mathrm{H} & 5.47339300 & -0.30593800 & 1.56273200 \\ \mathrm{C} & 4.19303400 & -3.64414600 & -0.59225700 \\ \mathrm{C} & 1.15076500 & -3.20216300 & -0.55572500 \\ \mathrm{C} & -2.40825200 & -3.52843100 & -0.01875000 \\ \mathrm{C} & -1.15453900 & -4.12087300 & -0.20824200 \\ \mathrm{H} & 7.21511000 & -0.00899900 & -0.19197200 \\ \mathrm{H} & 6.19953600 & -0.83290700 & -1.38843100 \\ \mathrm{H} & 5.70610100 & 0.73924500 & -0.74770400 \\ \mathrm{C} & 4.36834900 & -3.80815000 & -2.11494700 \\ \mathrm{H} & 3.66550900 & -4.52043400 & -0.19214700 \\ \mathrm{H} & 5.17865600 & -3.65188600 & -0.11477300 \\ \mathrm{H} & 1.56620000 & -4.19524800 & -0.68095400 \\ \mathrm{C} & -3.69386600 & -4.21219800 & 0.35468800 \\ \mathrm{C} & -0.80984600 & -5.57914600 & -0.08728900 \\ \mathrm{H} & 4.94051300 & -2.97494500 & -2.53613700 \\ \mathrm{H} & 3.40140100 & -3.83990900 & -2.62911300 \\ \mathrm{H} & 4.90327300 & -4.73629700 & -2.34108700 \\ \mathrm{C} & -4.03818300 & -4.09295000 & 1.85349500 \\ \mathrm{H} & -3.63395800 & -5.27020400 & 0.07926400 \\ \mathrm{H} & -4.51568300 & -3.79161100 & -0.23856700 \\ \mathrm{C} & -0.39034400 & -5.99662300 & 1.33717400 \\ \mathrm{H} & -0.00343600 & -5.82277500 & -0.78894300 \\ \mathrm{H} & -1.67140400 & -6.17871000 & -0.40122100 \\ \mathrm{H} & -4.13931100 & -3.04576900 & 2.15665100 \\ \mathrm{H} & -4.98345800 & -4.60246800 & 2.06482500 \\ \mathrm{H} & -3.26140500 & -4.54732500 & 2.47670600 \\ \mathrm{H} & -0.13806000 & -7.06143800 & 1.35851400 \\ \mathrm{H} & -1.19843700 & -5.82664900 & 2.05569900 \\ \mathrm{H} & 0.48386600 & -5.43110400 & 1.67578300 \\ \mathrm{H} & 3.92287100 & 3.36758100 & 2.23939900 \\ \mathrm{H} & 3.98594800 & 1.32143900 & 1.24830400 \\ \mathrm{~N} & -0.88781600 & -1.81058200 & -0.50232500 \\ \mathrm{H} & -0.86166800 & -1.49523500 & -1.48354400\end{array}$


Structure of bridge protonated vanadyl Octaethylporphyrin (OEP)

\begin{tabular}{|c|c|c|c|}
\hline $\mathrm{C}$ & -0.49769800 & -3.01309300 & 0.19526300 \\
\hline $\mathrm{C}$ & -0.96908900 & 3.28031200 & -0.31147700 \\
\hline $\mathrm{C}$ & 0.39560900 & 3.01996600 & -0.39152000 \\
\hline $\mathrm{C}$ & 1.41365600 & 4.01727200 & -0.62504400 \\
\hline $\mathrm{C}$ & 2.60904700 & 3.33114800 & -0.72474300 \\
\hline $\mathrm{C}$ & 2.28764200 & 1.93950200 & -0.53332100 \\
\hline $\mathrm{C}$ & 3.23597100 & 0.89301700 & -0.56720600 \\
\hline $\mathrm{C}$ & 3.01111100 & -0.44170300 & -0.36401000 \\
\hline $\mathrm{C}$ & 4.02442900 & -1.48013200 & -0.38278500 \\
\hline $\mathrm{C}$ & 3.37562700 & -2.65978000 & -0.09595100 \\
\hline $\mathrm{C}$ & 1.98208700 & -2.31403700 & 0.07072100 \\
\hline $\mathrm{C}$ & -1.51254800 & -4.04091700 & 0.16731600 \\
\hline $\mathrm{C}$ & -1.26055000 & -5.52196600 & 0.25729200 \\
\hline $\mathrm{C}$ & -0.94582700 & -6.18658700 & -1.09791600 \\
\hline $\mathrm{C}$ & -4.08790300 & -3.97213700 & -0.09770400 \\
\hline $\mathrm{C}$ & -4.58491800 & -4.07787300 & -1.55477800 \\
\hline $\mathrm{C}$ & -5.55541300 & 1.20852300 & -0.22316800 \\
\hline $\mathrm{C}$ & -6.15593700 & 0.99727000 & 1.18123500 \\
\hline $\mathrm{C}$ & -3.99348900 & 4.04456500 & -0.29682100 \\
\hline $\mathrm{C}$ & -4.07625400 & 4.72428700 & 1.08560000 \\
\hline $\mathrm{C}$ & 1.18409200 & 5.49927700 & -0.71954300 \\
\hline $\mathrm{C}$ & -2.71260700 & -3.38271300 & 0.02406600 \\
\hline $\mathrm{C}$ & 1.10896400 & 6.19686500 & 0.65429200 \\
\hline $\mathrm{C}$ & 3.98446800 & 3.89323500 & -0.95735900 \\
\hline $\mathrm{C}$ & 4.79114400 & 4.11014900 & 0.33867000 \\
\hline $\mathrm{C}$ & 5.48755600 & -1.26144500 & -0.63946600 \\
\hline $\mathrm{C}$ & 6.27867700 & -0.86147600 & 0.62377600 \\
\hline $\mathrm{C}$ & 3.96374700 & -4.03890000 & 0.03326500 \\
\hline $\mathrm{C}$ & 4.21630500 & -4.48041400 & 1.48878700 \\
\hline $\mathrm{C}$ & -2.39809400 & -1.96657100 & -0.02029800 \\
\hline $\mathrm{C}$ & -3.31125900 & -0.95078000 & -0.10504800 \\
\hline $\mathrm{C}$ & -3.05119800 & 0.43741600 & -0.14028300 \\
\hline $\mathrm{C}$ & -4.07145500 & 1.45250400 & -0.21336500 \\
\hline $\mathrm{C}$ & -3.40534300 & 2.66269400 & -0.24786200 \\
\hline $\mathrm{C}$ & -1.99622700 & 2.34746700 & -0.20967300 \\
\hline $\mathrm{H}$ & -1.26792500 & 4.32055000 & -0.38149300 \\
\hline $\mathrm{H}$ & 4.26028300 & 1.18664800 & -0.76924900 \\
\hline $\mathrm{H}$ & -0.43804500 & -5.71808800 & 0.95697100 \\
\hline $\mathrm{H}$ & -2.14004800 & -6.00732700 & 0.69508700 \\
\hline $\mathrm{H}$ & -1.77653200 & -6.06163600 & -1.79996200 \\
\hline $\mathrm{H}$ & -0.05298500 & -5.75585700 & -1.56614500 \\
\hline $\mathrm{H}$ & -0.77133200 & -7.25932300 & -0.96610700 \\
\hline $\mathrm{H}$ & -4.09147700 & -4.96932700 & 0.35572900 \\
\hline $\mathrm{H}$ & -4.80024200 & -3.37516700 & 0.48534700 \\
\hline $\mathrm{H}$ & -5.58788700 & -4.51545200 & -1.58178300 \\
\hline $\mathrm{H}$ & -4.62852600 & -3.09476300 & -2.03550400 \\
\hline $\mathrm{H}$ & -3.92154300 & -4.71099600 & -2.15265300 \\
\hline $\mathrm{H}$ & -6.05479300 & 2.05840400 & -0.70142400 \\
\hline $\mathrm{H}$ & -5.78694300 & 0.33953500 & -0.85215600 \\
\hline
\end{tabular}




$\begin{array}{lrrr}\mathrm{H} & -5.70927100 & 0.13157700 & 1.68296300 \\ \mathrm{H} & -5.98524700 & 1.87150000 & 1.81774700 \\ \mathrm{H} & -7.23611900 & 0.83153200 & 1.11404600 \\ \mathrm{H} & -3.40823700 & 4.67593000 & -0.97698300 \\ \mathrm{H} & -4.99803000 & 3.99295400 & -0.73088200 \\ \mathrm{H} & -4.69877200 & 4.14335100 & 1.77380900 \\ \mathrm{H} & -3.08534900 & 4.82620400 & 1.54144200 \\ \mathrm{H} & -4.51339700 & 5.72376700 & 0.99285800 \\ \mathrm{H} & 1.99083400 & 5.95144700 & -1.30697200 \\ \mathrm{H} & 0.26177800 & 5.69950200 & -1.27882000 \\ \mathrm{H} & 0.29311800 & 5.79325100 & 1.26381300 \\ \mathrm{H} & 2.03899800 & 6.06271100 & 1.21602200 \\ \mathrm{H} & 0.94071500 & 7.27117500 & 0.52713200 \\ \mathrm{H} & 4.54802100 & 3.23558400 & -1.63112200 \\ \mathrm{H} & 3.89543700 & 4.84857000 & -1.48636500 \\ \mathrm{H} & 5.77868700 & 4.52365300 & 0.10947100 \\ \mathrm{H} & 4.27843300 & 4.80702800 & 1.00957600 \\ \mathrm{H} & 4.93401700 & 3.17116100 & 0.88525300 \\ \mathrm{H} & 3.31155300 & -4.77304800 & -0.45837300 \\ \mathrm{H} & 4.91058800 & -4.07364200 & -0.51669200 \\ \mathrm{H} & 4.65676900 & -5.48239100 & 1.51198200 \\ \mathrm{H} & 4.90408000 & -3.79493400 & 1.99430300 \\ \mathrm{H} & 3.29008800 & -4.50948500 & 2.07373500 \\ \mathrm{H} & 5.91997000 & -2.17745100 & -1.05726600 \\ \mathrm{H} & 5.61843700 & -0.49116900 & -1.40914900 \\ \mathrm{H} & 7.33491600 & -0.71306100 & 0.37755100 \\ \mathrm{H} & 5.89555100 & 0.06788700 & 1.05880900 \\ \mathrm{H} & 6.21481400 & -1.63878000 & 1.39185400 \\ \mathrm{H} & -4.35590800 & -1.24025600 & -0.14531800 \\ \mathrm{~N} & -1.00738600 & -1.78423000 & 0.07721400 \\ \mathrm{~N} & -1.80048900 & 0.98357800 & -0.13575900 \\ \mathrm{~N} & 0.94995800 & 1.75762500 & -0.33102700 \\ \mathrm{~N} & 1.75476900 & -1.00709500 & -0.08823900 \\ \mathrm{O} & 0.05648700 & 0.17976000 & 1.95585200 \\ \mathrm{~V} & -0.00741800 & 0.04489300 & 0.40114500 \\ \mathrm{C} & 0.94063200 & -3.31670100 & 0.40955100 \\ \mathrm{H} & 1.06107400 & -3.56580500 & 1.48053400 \\ \mathrm{H} & 1.18146800 & -4.25520500 & -0.10302800\end{array}$


Structure of N-pyrrole protonated vanadyl Octaethylporphyrin (OEP)

\begin{tabular}{|c|c|c|c|}
\hline $\mathrm{C}$ & 2.91808700 & 1.14789200 & -0.02765200 \\
\hline C & -2.43640600 & -2.41513300 & -0.32924300 \\
\hline $\mathrm{C}$ & -2.88716200 & -1.09872900 & -0.3178910 \\
\hline $\mathrm{C}$ & -4.27614100 & -0.68632700 & -0.2750370 \\
\hline $\mathrm{C}$ & -4.27549400 & 0.69030600 & -0.274976 \\
\hline C & -2.88613000 & 1.10140700 & -0.317805 \\
\hline C & -2.43414400 & 2.41738900 & -0.3291010 \\
\hline $\mathrm{C}$ & -1.10862500 & 2.84094800 & -0.2972920 \\
\hline C & -0.69592300 & 4.23087100 & -0.2674420 \\
\hline $\mathrm{C}$ & 0.67672000 & 4.22980500 & -0.2071030 \\
\hline C & 1.08913400 & 2.83942700 & -0.2048000 \\
\hline $\mathrm{C}$ & 4.17708800 & 0.69916200 & 0.4352280 \\
\hline C & 2.42219100 & 2.43739200 & -0.0748390 \\
\hline C & 5.28902500 & 1.60497900 & 0.88576100 \\
\hline C & 6.16968500 & 2.10607600 & -0.2778320 \\
\hline $\mathrm{C}$ & 5.28754100 & -1.60995000 & 0.8858010 \\
\hline C & 6.16795500 & -2.11156600 & -0.2777520 \\
\hline $\mathrm{C}$ & 1.59618100 & -5.41506000 & -0.1270110 \\
\hline C & 2.00461500 & -5.78860100 & 1.312604 \\
\hline $\mathrm{C}$ & -1.63022400 & -5.41069200 & -0.266988 \\
\hline C & -2.18764000 & -5.75894400 & 1.1283570 \\
\hline C & -5.45387100 & -1.61697700 & -0.204220 \\
\hline C & 4.17643000 & -0.70312800 & 0.4352590 \\
\hline C & -5.76516200 & -2.11015400 & 1.2234950 \\
\hline $\mathrm{C}$ & -5.45234700 & 1.62206100 & -0.2040490 \\
\hline C & -5.76305200 & 2.11550300 & 1.2237020 \\
\hline $\mathrm{C}$ & -1.62510300 & 5.41220200 & -0.266972 \\
\hline C & -2.18194800 & 5.76122100 & 1.1284100 \\
\hline C & 1.60130300 & 5.41350300 & -0.12711500 \\
\hline C & 2.01017500 & 5.78667200 & 1.31247300 \\
\hline C & 2.91700500 & -1.15069200 & -0.0275970 \\
\hline C & 2.41989300 & -2.43972200 & -0.0747600 \\
\hline $\mathrm{C}$ & 1.08646200 & -2.84049600 & -0.2047790 \\
\hline C & 0.67272800 & -4.23048500 & -0.2070730 \\
\hline $\mathrm{C}$ & -0.69991100 & -4.23024900 & -0.267487 \\
\hline C & -1.11128900 & -2.83993600 & -0.2973920 \\
\hline $\mathrm{H}$ & -3.19412900 & -3.18961000 & -0.3431910 \\
\hline $\mathrm{H}$ & -3.19113500 & 3.19258300 & -0.3430040 \\
\hline $\mathrm{H}$ & 3.14414800 & 3.22975600 & 0.0857000 \\
\hline $\mathrm{H}$ & 4.86544100 & 2.46453900 & 1.4181750 \\
\hline $\mathrm{H}$ & 5.91666900 & 1.07956800 & 1.6131470 \\
\hline $\mathrm{H}$ & 6.64802600 & 1.27239600 & -0.8018860 \\
\hline $\mathrm{H}$ & 5.58180100 & 2.66809400 & -1.01136400 \\
\hline $\mathrm{H}$ & 6.95783200 & 2.76350700 & 0.1020980 \\
\hline $\mathrm{H}$ & 5.91551300 & -1.08517800 & 1.6133680 \\
\hline $\mathrm{H}$ & 4.86317700 & -2.46924900 & 1.4180140 \\
\hline $\mathrm{H}$ & 6.95550800 & -2.76971300 & 0.1021720 \\
\hline $\mathrm{H}$ & 5.57971500 & -2.67296900 & -1.011469 \\
\hline $\mathrm{H}$ & 6.64705500 & -1.27818400 & -0.801586 \\
\hline $\mathrm{H}$ & 1.10830900 & -6.27853400 & -0.5925890 \\
\hline $\mathrm{H}$ & 2.49604100 & -5.22748600 & -0.7255370 \\
\hline $\mathrm{H}$ & 2.51989900 & -4.96167300 & 1.8138630 \\
\hline
\end{tabular}




$\begin{array}{lrrr}\mathrm{H} & 1.12827700 & -6.04454200 & 1.91668900 \\ \mathrm{H} & 2.67632400 & -6.65315100 & 1.30634400 \\ \mathrm{H} & -2.46393600 & -5.22532800 & -0.95538900 \\ \mathrm{H} & -1.10242400 & -6.28277600 & -0.66847000 \\ \mathrm{H} & -1.38070600 & -6.00837500 & 1.82499900 \\ \mathrm{H} & -2.74826200 & -4.92122800 & 1.55732300 \\ \mathrm{H} & -2.85982600 & -6.62075500 & 1.06516300 \\ \mathrm{H} & -6.33683000 & -1.10985300 & -0.60856400 \\ \mathrm{H} & -5.28203700 & -2.48149600 & -0.85751300 \\ \mathrm{H} & -4.91408700 & -2.64803500 & 1.65539800 \\ \mathrm{H} & -5.99933200 & -1.27218400 & 1.88807300 \\ \mathrm{H} & -6.62587300 & -2.78666400 & 1.21387500 \\ \mathrm{H} & -5.27976400 & 2.48643000 & -0.85734200 \\ \mathrm{H} & -6.33582000 & 1.11577700 & -0.60832600 \\ \mathrm{H} & -6.62311800 & 2.79283400 & 1.21416600 \\ \mathrm{H} & -5.99796300 & 1.27774200 & 1.88828100 \\ \mathrm{H} & -4.91142800 & 2.65256400 & 1.65554500 \\ \mathrm{H} & 2.50095100 & 5.22506400 & -0.72568800 \\ \mathrm{H} & 1.11422900 & 6.27743900 & -0.59267700 \\ \mathrm{H} & 2.68279100 & 6.65051400 & 1.30615800 \\ \mathrm{H} & 1.13412600 & 6.04355500 & 1.91657600 \\ \mathrm{H} & 2.52460300 & 4.95921800 & 1.81374300 \\ \mathrm{H} & -1.09652800 & 6.28371000 & -0.66868500 \\ \mathrm{H} & -2.45910600 & 5.22751900 & -0.95520000 \\ \mathrm{H} & -2.85333900 & 6.62364900 & 1.06518200 \\ \mathrm{H} & -2.74327600 & 4.92410300 & 1.55762000 \\ \mathrm{H} & -1.37466000 & 6.01002000 & 1.82486600 \\ \mathrm{H} & 3.14107600 & -3.23277300 & 0.08585100 \\ \mathrm{~N} & -0.01274900 & -2.00566000 & -0.26139800 \\ \mathrm{~N} & -2.06133200 & 0.00095200 & -0.33834200 \\ \mathrm{~N} & -0.01087100 & 2.00563400 & -0.26133500 \\ \mathrm{~V} & -0.07785400 & 0.00003900 & -0.78068000 \\ \mathrm{O} & 0.02546700 & 0.00003400 & -2.34620600 \\ \mathrm{~N} & 2.12676200 & -0.00103700 & -0.41375600 \\ \mathrm{H} & 2.09642500 & -0.00106200 & -1.44609900\end{array}$


Structure of oxide protonated vanadyl Octaethylporphyrin (OEP)

\begin{tabular}{|c|c|c|c|}
\hline C & 3.04189000 & 0.35244100 & 0.00423700 \\
\hline $\mathrm{C}$ & -2.89204600 & -1.75147700 & -0.50136900 \\
\hline $\mathrm{C}$ & -2.96796700 & -0.37183300 & -0.54223000 \\
\hline C & -4.18917100 & 0.36593300 & -0.74975300 \\
\hline C & -3.85185400 & 1.69897400 & -0.72124100 \\
\hline C & -2.42815600 & 1.75732000 & -0.50180000 \\
\hline $\mathrm{C}$ & -1.70016200 & 2.93160900 & -0.4291990 \\
\hline $\mathrm{C}$ & -0.33081700 & 3.01445200 & -0.2621730 \\
\hline $\mathrm{C}$ & 0.41278600 & 4.24665800 & -0.2493470 \\
\hline $\mathrm{C}$ & 1.74197900 & 3.90571800 & -0.13113300 \\
\hline $\mathrm{C}$ & 1.79629100 & 2.46854500 & -0.07229800 \\
\hline $\mathrm{C}$ & 4.27629800 & -0.39083900 & 0.05020600 \\
\hline $\mathrm{C}$ & 2.96498600 & 1.73358500 & -0.00924200 \\
\hline $\mathrm{C}$ & 5.65294200 & 0.21289600 & 0.03697600 \\
\hline $\mathrm{C}$ & 6.14588000 & 0.59244000 & -1.37415100 \\
\hline $\mathrm{C}$ & 4.84323200 & -2.91717900 & 0.10265700 \\
\hline $\mathrm{C}$ & 5.10187800 & -3.52659900 & -1.29028600 \\
\hline $\mathrm{C}$ & 0.23772700 & -5.63813300 & 0.02011700 \\
\hline $\mathrm{C}$ & 0.47886500 & -6.07018500 & 1.48087200 \\
\hline $\mathrm{C}$ & -2.87121200 & -4.83774900 & -0.37543100 \\
\hline $\mathrm{C}$ & -3.61911100 & -5.02383900 & 0.96032700 \\
\hline $\mathrm{C}$ & -5.55069200 & -0.24455300 & -0.93222700 \\
\hline $\mathrm{C}$ & 3.93165600 & -1.72217800 & 0.07649100 \\
\hline $\mathrm{C}$ & -6.24587300 & -0.61249900 & 0.39425800 \\
\hline $\mathrm{C}$ & -4.75842900 & 2.89022900 & -0.85922000 \\
\hline $\mathrm{C}$ & -5.17636600 & 3.50742500 & 0.49078200 \\
\hline $\mathrm{C}$ & -0.18946700 & 5.62107700 & -0.33477100 \\
\hline $\mathrm{C}$ & -0.72922400 & 6.14170200 & 1.01285300 \\
\hline $\mathrm{C}$ & 2.93154500 & 4.82175400 & -0.05932600 \\
\hline $\mathrm{C}$ & 3.42237000 & 5.08435500 & 1.37894100 \\
\hline $\mathrm{C}$ & 2.49166200 & -1.77552000 & 0.03883200 \\
\hline $\mathrm{C}$ & 1.75728500 & -2.94648000 & 0.04362800 \\
\hline $\mathrm{C}$ & 0.37936300 & -3.02828200 & -0.05492600 \\
\hline $\mathrm{C}$ & -0.35849900 & -4.26457600 & -0.10827300 \\
\hline $\mathrm{C}$ & -1.68206900 & -3.92387600 & -0.27644900 \\
\hline $\mathrm{C}$ & -1.73375500 & -2.48472700 & -0.31539900 \\
\hline $\mathrm{H}$ & -3.81462400 & -2.30395000 & -0.62858000 \\
\hline $\mathrm{H}$ & -2.24431900 & 3.86184000 & -0.53487000 \\
\hline $\mathrm{H}$ & 3.89728200 & 2.28372600 & 0.01653700 \\
\hline $\mathrm{H}$ & 5.67646200 & 1.10077500 & 0.68125900 \\
\hline $\mathrm{H}$ & 6.35851100 & -0.49619400 & 0.4836060 \\
\hline $\mathrm{H}$ & 6.18942700 & -0.28632000 & -2.0257170 \\
\hline $\mathrm{H}$ & 5.48193400 & 1.32362800 & -1.8477400 \\
\hline $\mathrm{H}$ & 7.14958900 & 1.02676700 & -1.3228990 \\
\hline $\mathrm{H}$ & 5.80047600 & -2.62683200 & 0.54946000 \\
\hline $\mathrm{H}$ & 4.42815600 & -3.68687100 & 0.76511900 \\
\hline $\mathrm{H}$ & 5.76927900 & -4.39072000 & -1.20978800 \\
\hline $\mathrm{H}$ & 4.17059700 & -3.85798700 & -1.76220100 \\
\hline H & 5.57061100 & -2.79701600 & -1.9586330 \\
\hline
\end{tabular}




$\begin{array}{lrrr}\mathrm{H} & -0.42681400 & -6.36304000 & -0.46264700 \\ \mathrm{H} & 1.18314800 & -5.68683900 & -0.53417100 \\ \mathrm{H} & 1.16139400 & -5.38375400 & 1.99359200 \\ \mathrm{H} & -0.45881600 & -6.08941700 & 2.04576700 \\ \mathrm{H} & 0.91577600 & -7.07347800 & 1.51426800 \\ \mathrm{H} & -3.56853700 & -4.45961200 & -1.13323700 \\ \mathrm{H} & -2.54044100 & -5.81689100 & -0.73868600 \\ \mathrm{H} & -2.96182900 & -5.45293500 & 1.72360900 \\ \mathrm{H} & -3.99575900 & -4.06977000 & 1.34507100 \\ \mathrm{H} & -4.47152700 & -5.69804900 & 0.82839700 \\ \mathrm{H} & -6.18515900 & 0.45687200 & -1.48521200 \\ \mathrm{H} & -5.47485400 & -1.13819100 & -1.56387000 \\ \mathrm{H} & -5.65831900 & -1.33803100 & 0.96745300 \\ \mathrm{H} & -6.38602600 & 0.27192600 & 1.02436700 \\ \mathrm{H} & -7.23050200 & -1.04990100 & 0.19990600 \\ \mathrm{H} & -4.27404900 & 3.65718000 & -1.47618700 \\ \mathrm{H} & -5.65823900 & 2.59230900 & -1.40862800 \\ \mathrm{H} & -5.83471500 & 4.36718600 & 0.32950400 \\ \mathrm{H} & -5.71380400 & 2.77924500 & 1.10708000 \\ \mathrm{H} & -4.30634000 & 3.84819600 & 1.06285900 \\ \mathrm{H} & 3.75513600 & 4.40883600 & -0.65472400 \\ \mathrm{H} & 2.67594300 & 5.77751600 & -0.52950600 \\ \mathrm{H} & 4.28653700 & 5.75641400 & 1.37008000 \\ \mathrm{H} & 2.63764700 & 5.55004400 & 1.98431600 \\ \mathrm{H} & 3.72030500 & 4.15513000 & 1.87704800 \\ \mathrm{H} & 0.56453100 & 6.32042600 & -0.71184700 \\ \mathrm{H} & -0.99747400 & 5.62677800 & -1.07670300 \\ \mathrm{H} & -1.15132200 & 7.14463200 & 0.89288600 \\ \mathrm{H} & -1.51410800 & 5.48816500 & 1.40924800 \\ \mathrm{H} & 0.06791100 & 6.19690900 & 1.76162900 \\ \mathrm{H} & 2.30725500 & -3.87740600 & 0.10407000 \\ \mathrm{~N} & 1.94445800 & -0.49794300 & -0.00934900 \\ \mathrm{~N} & -0.46801500 & -1.93448300 & -0.16766600 \\ \mathrm{~N} & -1.88447000 & 0.48517400 & -0.39271900 \\ \mathrm{~N} & 0.51799000 & 1.91631800 & -0.12982800 \\ \mathrm{O} & -0.16876100 & 0.05143500 & 2.03316400 \\ \mathrm{~V} & -0.01645600 & 0.00622300 & 0.31761400 \\ \mathrm{H} & 0.05590700 & 0.71109600 & 2.70962100\end{array}$


Structure of bridge protonated titanyl Octaethylporphyrin (OEP)

$\begin{array}{llll}\mathrm{Ti} & 0.00374000 & 0.04776400 & 0.46403100\end{array}$

$\begin{array}{lllll}\text { C } & 3.08696600 & 0.22033200 & -0.09869000\end{array}$

$\begin{array}{llll}\text { C } & 4.17661600 & 1.16116500 & -0.19256800\end{array}$

$\begin{array}{llll}\text { C } & 3.59753000 & 2.41429500 & -0.27180700\end{array}$

$\begin{array}{llll}\mathrm{C} & 2.16756000 & 2.20394600 & -0.23109200\end{array}$

$\begin{array}{llll}\text { C } & 1.20147500 & 3.20251900 & -0.35467300\end{array}$

$\begin{array}{llll}\text { C } & -0.18428200 & 3.05161700 & -0.41319700\end{array}$

$\begin{array}{llll}\text { C } & -1.13188800 & 4.11767400 & -0.65059900\end{array}$

$\begin{array}{llll}\text { C } & -2.37881600 & 3.52285200 & -0.70824700\end{array}$

$\begin{array}{llll}\text { C } & -2.16157000 & 2.11269300 & -0.49527700\end{array}$

$\begin{array}{llll}\text { C } & -3.18112100 & 1.12899900 & -0.50382100\end{array}$

$\begin{array}{lllll}\text { C } & -3.05932700 & -0.22508300 & -0.32139800\end{array}$

$\begin{array}{llll}\text { C } & -4.14558900 & -1.18876300 & -0.35882200\end{array}$

$\begin{array}{llll}\text { C } & -3.58075300 & -2.42337800 & -0.12879000\end{array}$

$\begin{array}{llll}\text { C } & -2.16179000 & -2.18842900 & 0.03299500\end{array}$

$\begin{array}{llll}\text { C } & 0.28016100 & -3.06895800 & 0.16143500\end{array}$

$\begin{array}{llll}\text { C } & 1.22841200 & -4.16148100 & 0.13871800\end{array}$

$\begin{array}{llll}\text { C } & 2.47415700 & -3.58148000 & 0.05353900\end{array}$

$\begin{array}{llll}\text { C } & 2.25837600 & -2.14506200 & 0.02848700\end{array}$

$\begin{array}{llll}\mathrm{C} & 3.23799100 & -1.18718800 & -0.03721200\end{array}$

$\begin{array}{llll}\mathrm{N} & 1.88287800 & 0.85906100 & -0.11825000\end{array}$

$\begin{array}{llll}\mathrm{N} & -0.83786200 & 1.84093400 & -0.31486000\end{array}$

$\mathrm{N} \quad \begin{array}{llll}\mathrm{N} & -1.84827300 & -0.89628000 & -0.07867300\end{array}$

$\begin{array}{llll}\mathrm{N} & 0.87888400 & -1.87899500 & 0.09230900\end{array}$

$\begin{array}{lllll}\mathrm{C} & 5.63930800 & 0.81380000 & -0.18379700\end{array}$

$\begin{array}{llll}\text { C } & 6.21046500 & 0.57855000 & 1.22856400\end{array}$

$\begin{array}{llll}\text { C } & 4.28287700 & 3.74758000 & -0.36774500\end{array}$

$\begin{array}{lllll}\text { C } & 4.42853100 & 4.46386600 & 0.99027700\end{array}$

$\begin{array}{lllll}\text { C } & -0.79348600 & 5.57492400 & -0.78586200\end{array}$

$\begin{array}{lllll}\text { C } & & -3.71283800 & 4.18193600 & -0.92437100\end{array}$

$\begin{array}{llll}\text { C } & 0.87812000 & -5.62421500 & 0.18005900\end{array}$

C $\quad 0.56336900 \quad-6.23401500 \quad-1.20020400$

$\begin{array}{llll}\text { C } & 3.81079300 & -4.25817200 & -0.03857400\end{array}$

$\begin{array}{llll}\text { C } & 4.34895500 & -4.36660000 & -1.48024700\end{array}$

$\begin{array}{llll}\mathrm{H} & 1.57470300 & 4.21562600 & -0.45551900\end{array}$

$\begin{array}{lllll}\mathrm{H} & -4.18472300 & 1.49734200 & -0.68603500\end{array}$

$\mathrm{H} \quad 4.25999300 \quad-1.54959000 \quad-0.05733200$

$\begin{array}{llll}\mathrm{H} & 6.20011200 & 1.61998600 & -0.66745800\end{array}$

$\mathrm{H} \quad \begin{array}{llll}5.81348500 & -0.07644800 & -0.80023300\end{array}$

$\mathrm{H} \quad 3.73750600 \quad 4.39602500 \quad-1.06362300$

$\begin{array}{llll}\mathrm{H} & 5.27543200 & 3.60944200 & -0.80849800\end{array}$

$\begin{array}{lllll}\mathrm{H} & -1.57464600 & 6.07175800 & -1.37011400\end{array}$

$\begin{array}{llll}\mathrm{H} & 0.12925100 & 5.69004500 & -1.36654200\end{array}$

$\mathrm{H} \quad-3.56244100 \quad 5.12481100 \quad-1.45981000$

$\mathrm{H} \quad-4.32976200 \quad 3.56281100 \quad-1.58684800$

$\begin{array}{llll}\mathrm{H} & 0.02412700 & -5.78221700 & 0.84973200\end{array}$

$\mathrm{H} \quad \begin{array}{llll}1.70970800 & -6.17552400 & 0.63089300\end{array}$

$\begin{array}{llll}\mathrm{H} & 3.73346000 & -5.26144100 & 0.39222000\end{array}$

$\mathrm{H} \quad 4.53871600 \quad-3.72133700 \quad 0.58117700$ 


$\begin{array}{lrrr}\mathrm{H} & 5.69942700 & -0.24564700 & 1.73633600 \\ \mathrm{H} & 7.27559300 & 0.33578200 & 1.17509700 \\ \mathrm{H} & 6.09634600 & 1.46964000 & 1.85227700 \\ \mathrm{H} & 3.45343000 & 4.65165200 & 1.45039500 \\ \mathrm{H} & 4.93471300 & 5.42479300 & 0.86156000 \\ \mathrm{H} & 5.01425900 & 3.86323700 & 1.69192200 \\ \mathrm{H} & 0.31411400 & -7.29435800 & -1.10190300 \\ \mathrm{H} & -0.28265300 & -5.73464000 & -1.68434000 \\ \mathrm{H} & 1.42134200 & -6.14882300 & -1.87302800 \\ \mathrm{H} & 5.32198400 & -4.86553200 & -1.48518800 \\ \mathrm{H} & 4.46982700 & -3.37994200 & -1.93740500 \\ \mathrm{H} & 3.66880400 & -4.94369400 & -2.11290700 \\ \mathrm{C} & -4.26668400 & -3.75945000 & -0.04344700 \\ \mathrm{H} & -3.66324400 & -4.52263300 & -0.55068500 \\ \mathrm{H} & -5.20702700 & -3.71153700 & -0.60211800 \\ \mathrm{C} & -6.33121900 & -0.41915100 & 0.69516700 \\ \mathrm{H} & -7.37622200 & -0.18914000 & 0.46953700 \\ \mathrm{H} & -6.31330400 & -1.20984800 & 1.45029400 \\ \mathrm{H} & -5.87419100 & 0.47132400 & 1.13735300 \\ \mathrm{C} & -5.59185500 & -0.85709700 & -0.58604100 \\ \mathrm{H} & -6.09687700 & -1.73101700 & -1.01008700 \\ \mathrm{H} & -5.67502900 & -0.06774900 & -1.34198100 \\ \mathrm{C} & -0.63851000 & 6.29607500 & 0.56850400 \\ \mathrm{H} & 0.15559000 & 5.84574800 & 1.17202000 \\ \mathrm{H} & -0.39263200 & 7.35044700 & 0.41362500 \\ \mathrm{H} & -1.56358200 & 6.24481800 & 1.14950700 \\ \mathrm{C} & -4.56393500 & -4.22140000 & 1.39674100 \\ \mathrm{H} & -5.20951100 & -3.50594500 & 1.91382800 \\ \mathrm{H} & -5.06947900 & -5.19117500 & 1.38986500 \\ \mathrm{H} & -3.64925100 & -4.32662100 & 1.98914700 \\ \mathrm{C} & -4.48394100 & 4.46029100 & 0.38106700 \\ \mathrm{H} & -5.44380600 & 4.93772800 & 0.16427500 \\ \mathrm{H} & -4.68125400 & 3.53689600 & 0.93492900 \\ \mathrm{H} & -3.91651000 & 5.12346700 & 1.04014300 \\ \mathrm{O} & -0.05539600 & 0.17879700 & 2.05646500 \\ \mathrm{C} & -1.18663200 & -3.27745800 & 0.31549800 \\ \mathrm{H} & -1.47377300 & -4.15576800 & -0.27431300 \\ \mathrm{H} & -1.35506500 & -3.60138600 & 1.35835400\end{array}$


Structure of N-pyrrole protonated titanyl Octaethylporphyrin (OEP)

\begin{tabular}{|c|c|c|c|}
\hline $\mathrm{C}$ & -2.79844400 & -1.32627900 & -0.04003500 \\
\hline $\mathrm{C}$ & 2.22203300 & 2.55652800 & -0.35954000 \\
\hline $\mathrm{C}$ & 2.73106900 & 1.26295000 & -0.49718700 \\
\hline $\mathrm{C}$ & 4.13975200 & 0.94708900 & -0.65977800 \\
\hline $\mathrm{C}$ & 4.23288700 & -0.42325900 & -0.72473800 \\
\hline $\mathrm{C}$ & 2.88128600 & -0.93727200 & -0.60390700 \\
\hline $\mathrm{C}$ & 2.56471100 & -2.30073700 & -0.53456800 \\
\hline $\mathrm{C}$ & 1.31710500 & -2.88379800 & -0.37742300 \\
\hline $\mathrm{C}$ & 0.99800300 & -4.18970600 & 0.06723500 \\
\hline $\mathrm{C}$ & -0.39409900 & -4.28333200 & 0.21396600 \\
\hline $\mathrm{C}$ & -0.96910100 & -3.03850100 & -0.13937100 \\
\hline $\mathrm{C}$ & -4.20494800 & -1.00105300 & 0.10916200 \\
\hline $\mathrm{C}$ & -2.29001300 & -2.63212100 & -0.03716300 \\
\hline $\mathrm{C}$ & -5.32768100 & -1.99958800 & 0.14923700 \\
\hline $\mathrm{C}$ & -5.74480000 & -2.51224100 & -1.24395500 \\
\hline $\mathrm{C}$ & -5.51768100 & 1.22111600 & 0.31040300 \\
\hline $\mathrm{C}$ & -6.02777900 & 1.77639200 & -1.03495900 \\
\hline $\mathrm{C}$ & -1.94362500 & 5.28017800 & 0.52853000 \\
\hline $\mathrm{C}$ & -2.30923400 & 5.38242500 & 2.02331700 \\
\hline $\mathrm{C}$ & 1.26905200 & 5.50366100 & 0.18647600 \\
\hline $\mathrm{C}$ & 1.92345200 & 5.67571300 & 1.57236600 \\
\hline $\mathrm{C}$ & 5.25446900 & 1.95465900 & -0.70263300 \\
\hline $\mathrm{C}$ & -4.28617800 & 0.37012100 & 0.17669900 \\
\hline $\mathrm{C}$ & 5.72430800 & 2.41752800 & 0.69158500 \\
\hline $\mathrm{C}$ & 5.47287000 & -1.26391900 & -0.85880600 \\
\hline $\mathrm{C}$ & 6.03589500 & -1.75995200 & 0.48854500 \\
\hline $\mathrm{C}$ & 2.01723000 & -5.24131300 & 0.40902200 \\
\hline $\mathrm{C}$ & 2.59890100 & -5.10283600 & 1.83105100 \\
\hline $\mathrm{C}$ & -1.17252200 & -5.45531400 & 0.74501800 \\
\hline $\mathrm{C}$ & -1.47968700 & -5.36335500 & 2.25412600 \\
\hline $\mathrm{C}$ & -2.92834900 & 0.87516700 & 0.06959100 \\
\hline $\mathrm{C}$ & -2.57913200 & 2.22596500 & 0.14021700 \\
\hline $\mathrm{C}$ & -1.30092100 & 2.78199800 & 0.06433200 \\
\hline $\mathrm{C}$ & -0.96228500 & 4.18397200 & 0.22302300 \\
\hline $\mathrm{C}$ & 0.40534700 & 4.27897500 & 0.07695700 \\
\hline $\mathrm{C}$ & 0.89140000 & 2.93378500 & -0.16789200 \\
\hline $\mathrm{H}$ & 2.94941500 & 3.35986900 & -0.37844900 \\
\hline $\mathrm{H}$ & 3.40882800 & -2.98140000 & -0.50966100 \\
\hline $\mathrm{H}$ & -3.01060300 & -3.42006400 & 0.15482900 \\
\hline $\mathrm{H}$ & -5.04663900 & -2.85274300 & 0.78055200 \\
\hline $\mathrm{H}$ & -6.19566800 & -1.54457500 & 0.63844800 \\
\hline $\mathrm{H}$ & -6.08596500 & -1.68866000 & -1.87954500 \\
\hline $\mathrm{H}$ & -4.91048800 & -3.00288100 & -1.75700800 \\
\hline $\mathrm{H}$ & -6.56255400 & -3.23489800 & -1.15580700 \\
\hline $\mathrm{H}$ & -6.31309600 & 0.63276700 & 0.78066200 \\
\hline $\mathrm{H}$ & -5.32006500 & 2.05452200 & 0.99624200 \\
\hline $\mathrm{H}$ & -6.92145000 & 2.38940300 & -0.87948400 \\
\hline $\mathrm{H}$ & -5.26971200 & 2.39634300 & -1.52562700 \\
\hline $\mathrm{H}$ & -6.28766500 & 0.96461700 & -1.72210400 \\
\hline $\mathrm{H}$ & -1.52836100 & 6.23733000 & 0.19445600 \\
\hline $\mathrm{H}$ & -2.85806300 & 5.13043700 & -0.05929400 \\
\hline $\mathrm{H}$ & -2.75769900 & 4.45280200 & 2.39046300 \\
\hline $\mathrm{H}$ & -1.42302600 & 5.58664300 & 2.63304800 \\
\hline
\end{tabular}




$\begin{array}{crrr}\mathrm{H} & -3.02680000 & 6.19326900 & 2.18525100 \\ \mathrm{H} & 2.05058600 & 5.47385400 & -0.58317400 \\ \mathrm{H} & 0.66470900 & 6.38971500 & -0.03689300 \\ \mathrm{H} & 1.16605200 & 5.76275200 & 2.35825500 \\ \mathrm{H} & 2.56372800 & 4.82233700 & 1.82187200 \\ \mathrm{H} & 2.53966100 & 6.58037500 & 1.59302900 \\ \mathrm{H} & 6.10560800 & 1.52371500 & -1.24124900 \\ \mathrm{H} & 4.94216000 & 2.82731800 & -1.28939300 \\ \mathrm{H} & 4.90811900 & 2.87911500 & 1.25808500 \\ \mathrm{H} & 6.10093000 & 1.57521100 & 1.28092100 \\ \mathrm{H} & 6.53017600 & 3.15246300 & 0.59686000 \\ \mathrm{H} & 5.26907100 & -2.12396600 & -1.50845300 \\ \mathrm{H} & 6.24470200 & -0.68184400 & -1.37434300 \\ \mathrm{H} & 6.93434100 & -2.36436400 & 0.32702300 \\ \mathrm{H} & 6.30420700 & -0.91973400 & 1.13695400 \\ \mathrm{H} & 5.30698300 & -2.37411700 & 1.02942700 \\ \mathrm{H} & -2.11425300 & -5.54491700 & 0.18972700 \\ \mathrm{H} & -0.61658700 & -6.37734100 & 0.54464300 \\ \mathrm{H} & -2.05458000 & -6.23773100 & 2.57466700 \\ \mathrm{H} & -0.55992000 & -5.32783300 & 2.84632200 \\ \mathrm{H} & -2.06284900 & -4.46679500 & 2.48829500 \\ \mathrm{H} & 1.56247200 & -6.23161700 & 0.30009800 \\ \mathrm{H} & 2.83458900 & -5.20363500 & -0.32134100 \\ \mathrm{H} & 3.34027100 & -5.88728100 & 2.01210600 \\ \mathrm{H} & 3.08765800 & -4.13276900 & 1.96919200 \\ \mathrm{H} & 1.81742700 & -5.19429500 & 2.59189200 \\ \mathrm{H} & -3.39624200 & 2.92177800 & 0.29213900 \\ \mathrm{~N} & -2.04132200 & -0.17506600 & -0.06665400 \\ \mathrm{~N} & -0.16157300 & 2.05257900 & -0.17169600 \\ \mathrm{~N} & 1.98477000 & 0.10066900 & -0.46827000 \\ \mathrm{O} & -0.2485800 & 0.02739100 & -2.40839300 \\ \mathrm{Ti} & -0.09173000 & 0.09675200 & -0.80975000 \\ \mathrm{~N} & 0.08464600 & -2.16219800 & -0.59831800 \\ \mathrm{H} & -0.02802500 & -2.05460200 & -1.62083200\end{array}$


Structure of oxide protonated titanyl Octaethylporphyrin (OEP)

\begin{tabular}{|c|c|c|c|}
\hline Ti & 0.02442200 & 0.00425400 & 0.37207000 \\
\hline $\mathrm{C}$ & -0.76512700 & -2.96879800 & -0.08021400 \\
\hline $\mathrm{C}$ & -0.18868200 & -4.28838200 & -0.16658700 \\
\hline $\mathrm{C}$ & 1.16955800 & -4.11868400 & -0.31042300 \\
\hline $\mathrm{C}$ & 1.41308600 & -2.69673500 & -0.30567000 \\
\hline $\mathrm{C}$ & 2.65869800 & -2.10882700 & -0.45699800 \\
\hline $\mathrm{C}$ & 2.92009500 & -0.74801000 & -0.49035300 \\
\hline $\mathrm{C}$ & 4.22519800 & -0.16785000 & -0.69291600 \\
\hline $\mathrm{C}$ & 4.05580800 & 1.19824600 & -0.68948000 \\
\hline $\mathrm{C}$ & 2.64845700 & 1.44130500 & -0.48527400 \\
\hline $\mathrm{C}$ & 2.06164000 & 2.69678900 & -0.45062500 \\
\hline $\mathrm{C}$ & 0.70894900 & 2.96147300 & -0.30410300 \\
\hline $\mathrm{C}$ & 0.12529300 & 4.28072400 & -0.30752100 \\
\hline $\mathrm{C}$ & -1.23394600 & 4.11237300 & -0.16941400 \\
\hline $\mathrm{C}$ & -1.46998900 & 2.69152400 & -0.08635600 \\
\hline $\mathrm{C}$ & -2.72024500 & 2.10268000 & 0.01850500 \\
\hline $\mathrm{C}$ & -2.97960900 & 0.74178600 & 0.05017600 \\
\hline $\mathrm{C}$ & -4.29754700 & 0.15965300 & 0.10136800 \\
\hline $\mathrm{C}$ & -4.12794400 & -1.20674200 & 0.10509200 \\
\hline $\mathrm{C}$ & -2.70724300 & -1.44904200 & 0.05298400 \\
\hline $\mathrm{C}$ & -2.12172800 & -2.70479500 & 0.02395500 \\
\hline $\mathrm{N}$ & 0.22238500 & -1.99604000 & -0.15945700 \\
\hline $\mathrm{N}$ & 1.95752600 & 0.24366700 & -0.36102100 \\
\hline $\mathrm{N}$ & -0.27427000 & 1.99059200 & -0.16252500 \\
\hline $\mathrm{N}$ & -2.00566100 & -0.24983900 & 0.02271300 \\
\hline $\mathrm{C}$ & -0.96093100 & -5.57546200 & -0.09427100 \\
\hline $\mathrm{C}$ & -1.29608900 & -6.01563000 & 1.34435300 \\
\hline $\mathrm{C}$ & 2.23034700 & -5.17671000 & -0.43192900 \\
\hline $\mathrm{C}$ & 2.93699800 & -5.49939900 & 0.89991800 \\
\hline $\mathrm{C}$ & 5.50191900 & -0.94509000 & -0.85283400 \\
\hline $\mathrm{C}$ & 5.10385100 & 2.26418900 & -0.84553300 \\
\hline $\mathrm{C}$ & -5.58496200 & 0.93525900 & 0.11196400 \\
\hline $\mathrm{C}$ & -6.04976900 & 1.38353000 & -1.28796400 \\
\hline $\mathrm{C}$ & -5.18628700 & -2.27417000 & 0.11569300 \\
\hline $\mathrm{C}$ & -5.52330600 & -2.82465400 & -1.28455400 \\
\hline $\mathrm{H}$ & 3.50467300 & -2.77376800 & -0.57720300 \\
\hline $\mathrm{H}$ & 2.71892900 & 3.54921100 & -0.56655800 \\
\hline $\mathrm{H}$ & -3.57458900 & 2.76651800 & 0.05475000 \\
\hline $\mathrm{H}$ & -2.78741900 & -3.55769200 & 0.05988800 \\
\hline $\mathrm{H}$ & -0.38361100 & -6.36585900 & -0.58402100 \\
\hline $\mathrm{H}$ & -1.88790900 & -5.48285900 & -0.67245000 \\
\hline $\mathrm{H}$ & 2.97575200 & -4.86874400 & -1.17428600 \\
\hline $\mathrm{H}$ & 1.77830700 & -6.09153400 & -0.82794800 \\
\hline $\mathrm{H}$ & 6.22739600 & -0.33257000 & -1.39766300 \\
\hline $\mathrm{H}$ & 5.32316200 & -1.82347000 & -1.48376800 \\
\hline $\mathrm{H}$ & 5.95676700 & 1.84912900 & -1.39205400 \\
\hline $\mathrm{H}$ & 4.71544300 & 3.07436400 & -1.47368400 \\
\hline $\mathrm{H}$ & -5.48088200 & 1.81386500 & 0.75962900 \\
\hline $\mathrm{H}$ & -6.36716000 & 0.32082900 & 0.56909900 \\
\hline $\mathrm{H}$ & -6.09652200 & -1.86981800 & 0.56999600 \\
\hline $\mathrm{H}$ & -4.87145200 & -3.09931100 & 0.76533800 \\
\hline $\mathrm{H}$ & -1.89980500 & -5.26472300 & 1.86348700 \\
\hline $\mathrm{H}$ & -1.85749500 & -6.95425600 & 1.33520300 \\
\hline
\end{tabular}




$\begin{array}{lrrr}\mathrm{H} & -0.38553600 & -6.17160200 & 1.93021900 \\ \mathrm{H} & 3.42869200 & -4.61460100 & 1.31622000 \\ \mathrm{H} & 3.69699600 & -6.27192900 & 0.75212300 \\ \mathrm{H} & 2.22501800 & -5.86387100 & 1.64617900 \\ \mathrm{H} & -6.98684700 & 1.94323600 & -1.21803300 \\ \mathrm{H} & -5.30600000 & 2.02460500 & -1.77074800 \\ \mathrm{H} & -6.21777400 & 0.52300200 & -1.94159900 \\ \mathrm{H} & -6.29814700 & -3.59347000 & -1.21573200 \\ \mathrm{H} & -4.64549600 & -3.26922500 & -1.76307300 \\ \mathrm{H} & -5.89107300 & -2.03135400 & -1.94154300 \\ \mathrm{C} & -2.29839100 & 5.16992900 & -0.09933600 \\ \mathrm{H} & -3.17309500 & 4.85165100 & -0.67829300 \\ \mathrm{H} & -1.93264700 & 6.07725000 & -0.59045100 \\ \mathrm{C} & 1.50058400 & 6.05005100 & 0.90567400 \\ \mathrm{H} & 2.05043900 & 6.98394700 & 0.75797100 \\ \mathrm{H} & 0.72051800 & 6.23033700 & 1.65096000 \\ \mathrm{H} & 2.19203500 & 5.31096700 & 1.32211900 \\ \mathrm{C} & 0.89509000 & 5.56576100 & -0.42631800 \\ \mathrm{H} & 0.23450500 & 6.34294700 & -0.82336500 \\ \mathrm{H} & 1.69437000 & 5.44891500 & -1.16747100 \\ \mathrm{C} & 6.12199800 & -1.39179900 & 0.48593200 \\ \mathrm{H} & 5.43726900 & -2.03221700 & 1.05040300 \\ \mathrm{H} & 7.04512900 & -1.95182400 & 0.31196400 \\ \mathrm{H} & 6.36210900 & -0.52942200 & 1.11468700 \\ \mathrm{C} & -2.73432600 & 5.51561800 & 1.33858900 \\ \mathrm{H} & -1.89062000 & 5.88838400 & 1.92659300 \\ \mathrm{H} & -3.50809900 & 6.28840700 & 1.32836600 \\ \mathrm{H} & -3.13721600 & 4.63916500 & 1.85585000 \\ \mathrm{C} & 5.59594500 & 2.84500900 & 0.49445300 \\ \mathrm{H} & 6.35358000 & 3.61475300 & 0.32229600 \\ \mathrm{H} & 4.77602100 & 3.29663900 & 1.06136100 \\ \mathrm{H} & 6.04096100 & 2.06678000 & 1.12123700 \\ \mathrm{O} & 0.18280600 & 0.02460800 & 2.10202900 \\ \mathrm{H} & 0.05738100 & -0.03062900 & 3.05436800\end{array}$




\section{Appendix 5.1}

The Collisional Cross Section in nitrogen, relative energy and names for structures considered for $\mathrm{C}_{10} \mathrm{H}_{8}$.

\begin{tabular}{|c|c|c|}
\hline $\operatorname{CCS} \AA^{2}$ & $\begin{array}{l}\text { Relative Energy } \\
\quad \text { (kcal/mol) }\end{array}$ & Name \\
\hline 115.56 & 0 & Napthalene \\
\hline 117.18 & 26 & 1-methyleneindene \\
\hline 115.99 & 36 & Azulene \\
\hline 114.46 & 48 & 2-Methylene-2H-indene \\
\hline 127.64 & 56 & 1-phenyl-1-butyne-3-ene \\
\hline 119.39 & 59 & 4-Phenylbut-3-ene-1-yne \\
\hline 119.39 & 59 & 4-Phenylbut-3-ene-1-yne \\
\hline 121.62 & 60 & (1Z)-1-Buten-3-yn-1-ylbenzene \\
\hline 111.79 & 61 & 1-Ethynyl-4-vinylbenzene \\
\hline 125.64 & 61 & 1-Ethynyl-3-vinylbenzene \\
\hline 122.18 & 63 & 1-Buten-3-yn-2-ylbenzene \\
\hline 118.20 & 64 & 1-Ethynyl-2-vinylbenzene \\
\hline 117.92 & 64 & 1,1'-Bi(2,4-cyclopentadien-1-ylidene) \\
\hline 118.78 & 75 & bicyclo[4.4.0]deca-1,3,5,7,8-pentaene \\
\hline 118.63 & 77 & 3-Ethynylbicyclo[4.2.0]octa-1,3,5-triene \\
\hline 121.53 & 80 & Tricyclo[5.2.1.0.0.6 $]$ deca-3,8-diene-3,4,8,9-tetraide \\
\hline 124.37 & 84 & Tricyclo[4.4.0.0 $\left.0^{2,5}\right]$ deca-1(10),3,6,8-tetraene \\
\hline 117.46 & 87 & 4-Vinylbenzocyclobutene \\
\hline 123.30 & 96 & 5,6-Diethenylidene-1,3-cyclohexadiene \\
\hline 118.90 & 97 & phenylcyclobutadiene \\
\hline 125.97 & 103 & 1-Ethynyl-1,3,5,7-cyclooctatetraene \\
\hline 113.81 & 111 & 1,4-Diethynyl-1,3-cyclohexadiene \\
\hline 125.10 & 113 & 7-ethynylbicyclo[4.2.0]octa-2,4,7-triene \\
\hline 112.78 & 114 & 1,4-Diethynyl-1,4-cyclohexadiene \\
\hline 122.68 & 115 & 1,2-Diethynyl-1,4-cyclohexadiene \\
\hline 120.92 & 116 & $\begin{array}{l}\text { (5Z)-1-Ethynyl-5-(2-propyn-1- } \\
\text { ylidene)cyclopentene }\end{array}$ \\
\hline 132.89 & 122 & 2,5-Diethynyl-1,3-cyclohexadiene \\
\hline 125.46 & 124 & Tricyclo[4.2.2.0 $\left.0^{1,6}\right] \mathrm{deca}-2,4,7,9$-tetraene \\
\hline 122.17 & 131 & 3,6-Diethynyl-1,4-cyclohexadiene \\
\hline 122.17 & 131 & trans-3,6-Diethynyl-1,4-cyclohexadiene \\
\hline 123.12 & 138 & Bicyclo[4.2.2]deca-1,3,5,7,9-pentaene \\
\hline 123.33 & 145 & $(1 Z, 3 Z, 5 Z, 7 Z)-1,3,5,7-C y c l o d e c a t e t r a e n-9-y n e$ \\
\hline 119.76 & 158 & (4Z,9Z)-1,2,4,6,7,9-Cyclodecahexaene \\
\hline 126.09 & 178 & Tricyclo[5.2.1.0.0 $\left.0^{2,6}\right]$ deca-3,8-diene-3,4,8,9-tetraide \\
\hline 123.61 & 193 & 1-Ethynylcubane \\
\hline
\end{tabular}




\section{Appendix 5.2}

The Collisional Cross Section in nitrogen, relative energy and names for structures considered for $\mathrm{C}_{12} \mathrm{H}_{8}$.

$\begin{array}{lcr} & \text { Relative } & \\ \text { CCS } & \text { Energy } & \text { Name } \\ {\left[\AA^{2}\right]} & (\mathrm{kcal} / \mathrm{mol}) & \end{array}$

\begin{tabular}{|c|c|c|}
\hline 123.88 & 0 & Acenaphthylene \\
\hline 123.33 & 28 & 2-ethynyl-naphtalene \\
\hline 127.22 & 28 & 1-Ethynylnaphthalene \\
\hline 124.01 & 32 & Cyclopenta[a]indene \\
\hline 126.86 & 38 & Biphenylene \\
\hline 121.39 & 39 & s-Indacene \\
\hline 123.95 & 48 & as-Indacene \\
\hline 142.08 & 82 & (3E)-3-Hexene-1,5-diyn-1-ylbenzene \\
\hline 142.09 & 82 & (3E)-3-Hexene-1,5-diyn-1-ylbenzene \\
\hline 135.27 & 91 & 3,5-diethynylstyrene \\
\hline 128.35 & 98 & cyclohexatrienylcyclohexatriene \\
\hline 130.02 & 130 & 1-Ethynylazulene \\
\hline 132.12 & 141 & Smiles: $\mathrm{CC} 1=\mathrm{C}=\mathrm{CC} 2=\mathrm{C} 1 \mathrm{c} 3 \mathrm{c} 2 \mathrm{ccc} 3$ \\
\hline 147.77 & 160 & 5-Isopropylidene-1,3,6,8-nonatetrayne \\
\hline 119.75 & 179 & 1,4-Dihydrodicyclopropa[b,g]naphthalene \\
\hline 142.39 & 179 & $\begin{array}{l}\text { 3-ethenyl-1,5-diethynylbicyclo[2.2.0]hexa-2,5- } \\
\text { diene }\end{array}$ \\
\hline 125.71 & 197 & 1,2-Dihydroacenaphthylene-4,5-diide \\
\hline 133.31 & 202 & Smiles: $\mathrm{C} 2 \# \mathrm{CC}=\mathrm{CC} 1 \mathrm{C}=\mathrm{C} 1 \mathrm{C} 34 \mathrm{CC} 24 \mathrm{C}=\mathrm{C} 3$ \\
\hline 126.84 & 219 & 1,4-Diethynylcubane \\
\hline 137.63 & 436 & 2-cyclopentylethynylcyclopentane \\
\hline
\end{tabular}




\section{Appendix 6.1}

Relative abundance of the oxygen containing series $\left(\mathrm{CxHyO}_{1-15}\right)$ identified in the SRFA sample.

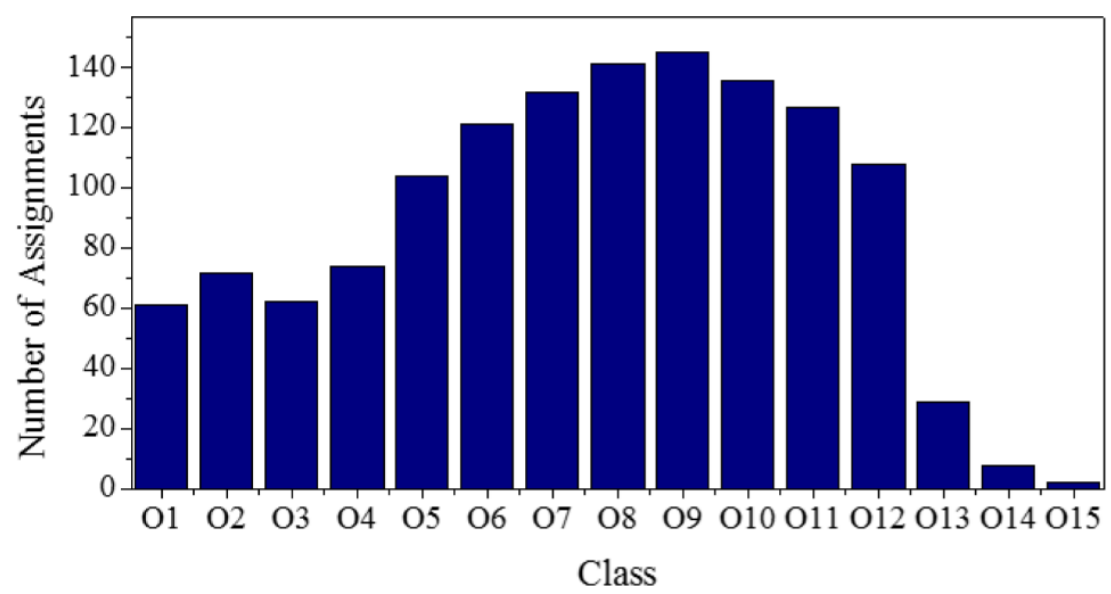




\section{Appendix 6.2}

Double Bond Equivalents (DBE) as a function of the carbon number for the oxygen containing series $\left(\mathrm{CxHyO}_{1-15}\right)$ identified in the SRFA sample.
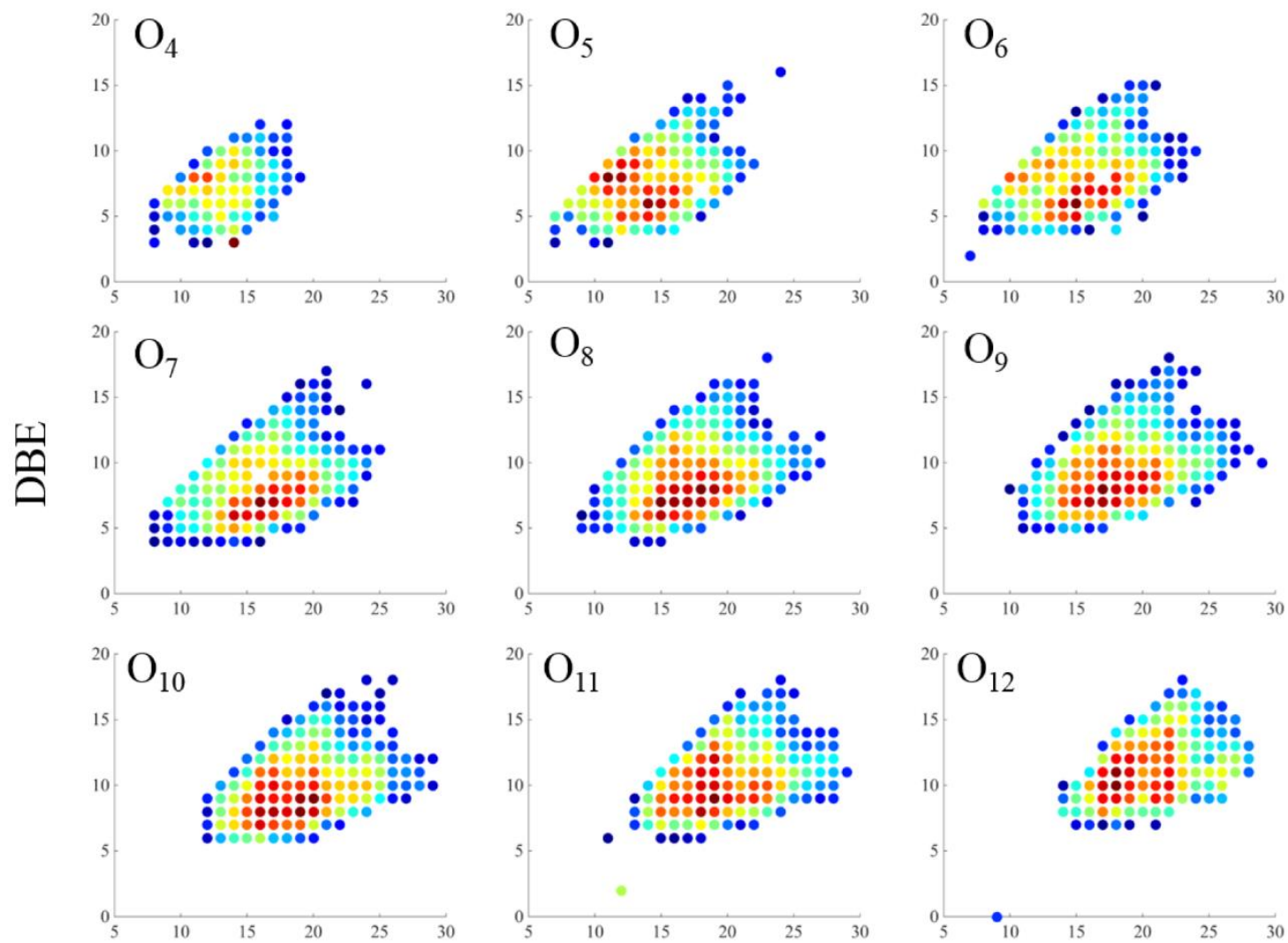

Carbon Number 


\section{Appendix 6.3}

Candidate structures geometry and atomic charges for the EDC targeted compounds.

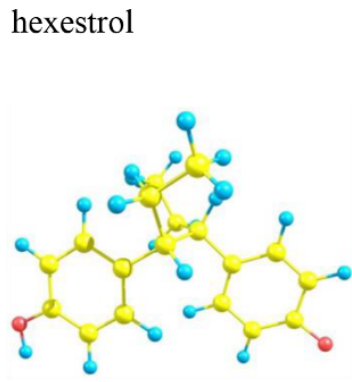

\begin{tabular}{|c|c|c|c|c|}
\hline Atom & & Coordinates & & Partial Charges \\
\hline $\mathrm{C}$ & 0.8034 & 2.15838 & 2.24896 & -0.108 \\
\hline $\mathrm{C}$ & -0.21698 & 3.37566 & -1.59239 & -0.19335 \\
\hline $\mathrm{O}$ & 4.8143 & -2.37175 & 0.16052 & -0.58302 \\
\hline $\mathrm{C}$ & 1.53285 & 0.11356 & -0.51033 & 0.1909 \\
\hline $\mathrm{C}$ & 2.74572 & 0.63684 & -0.04164 & -0.25453 \\
\hline $\mathrm{C}$ & 1.41577 & -1.24955 & -0.77242 & -0.25405 \\
\hline $\mathrm{C}$ & 3.82829 & -0.21481 & 0.15241 & -0.19728 \\
\hline $\mathrm{C}$ & 2.4834 & -2.10793 & -0.55404 & -0.24588 \\
\hline $\mathrm{C}$ & 3.70626 & -1.58804 & -0.10714 & 0.33254 \\
\hline $\mathrm{C}$ & 0.37166 & 1.02882 & -0.7567 & -0.11688 \\
\hline $\mathrm{C}$ & -0.6334 & 1.14454 & 0.43539 & 0.0906 \\
\hline $\mathrm{O}$ & -5.18129 & -2.17482 & -0.21793 & -0.85452 \\
\hline $\mathrm{C}$ & -4.03312 & -1.4351 & -0.06098 & 0.50131 \\
\hline $\mathrm{C}$ & -4.19282 & -0.03789 & -0.06441 & -0.18143 \\
\hline $\mathrm{C}$ & -2.76328 & -2.00894 & 0.10471 & -0.21694 \\
\hline $\mathrm{C}$ & -3.07505 & 0.76602 & 0.0895 & -0.3696 \\
\hline $\mathrm{C}$ & -1.65919 & -1.18418 & 0.27951 & -0.39997 \\
\hline $\mathrm{C}$ & -1.79769 & 0.20792 & 0.24294 & 0.15352 \\
\hline $\mathrm{C}$ & 0.87728 & 2.43308 & -1.15482 & 0.25519 \\
\hline $\mathrm{C}$ & -0.00855 & 0.95028 & 1.82994 & 0.00863 \\
\hline $\mathrm{H}$ & -0.17632 & 0.60017 & -1.63563 & 0.02301 \\
\hline $\mathrm{H}$ & -1.04883 & 2.19181 & 0.39931 & 0.0272 \\
\hline $\mathrm{H}$ & 1.3658 & 1.98634 & 3.17437 & 0.00238 \\
\hline $\mathrm{H}$ & 1.54628 & 2.38337 & 1.4554 & 0.02363 \\
\hline $\mathrm{H}$ & 0.18808 & 3.05168 & 2.39506 & 0.00295 \\
\hline $\mathrm{H}$ & -0.79084 & 2.98863 & -2.43923 & 0.0401 \\
\hline $\mathrm{H}$ & -0.93574 & 3.52604 & -0.76616 & 0.02962 \\
\hline $\mathrm{H}$ & 0.17098 & 4.36532 & -1.86486 & 0.01117 \\
\hline $\mathrm{H}$ & 4.5845 & -3.26623 & -0.06084 & 0.38571 \\
\hline $\mathrm{H}$ & 2.79821 & 1.71399 & 0.20467 & 0.13639 \\
\hline $\mathrm{H}$ & 0.4566 & -1.64321 & -1.14255 & 0.16813 \\
\hline $\mathrm{H}$ & 4.75423 & 0.19555 & 0.56934 & 0.13926 \\
\hline $\mathrm{H}$ & 2.37686 & -3.16523 & -0.81444 & 0.1224 \\
\hline $\mathrm{H}$ & -5.1733 & 0.40376 & -0.28478 & 0.09234 \\
\hline $\mathrm{H}$ & -2.63893 & -3.09578 & 0.15073 & 0.08216 \\
\hline $\mathrm{H}$ & -3.15793 & 1.85975 & 0.01296 & 0.10605 \\
\hline $\mathrm{H}$ & -0.64955 & -1.62231 & 0.35621 & 0.17303 \\
\hline $\mathrm{H}$ & 1.63604 & 2.33815 & -1.9592 & -0.0789 \\
\hline $\mathrm{H}$ & 1.43387 & 2.86084 & -0.28286 & -0.06256 \\
\hline $\mathrm{H}$ & 0.66424 & 0.0692 & 1.79637 & 0.02509 \\
\hline $\mathrm{H}$ & -0.77556 & 0.69885 & 2.58441 & -0.00641 \\
\hline
\end{tabular}


$\alpha$-estradiol

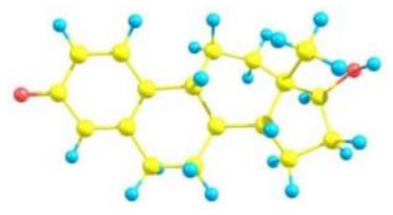

\begin{tabular}{lrrrr} 
& \multicolumn{3}{c}{ Partial } \\
Atom & \multicolumn{3}{c}{ Coordinates } & \multicolumn{1}{c}{ Charges } \\
\hline $\mathrm{O}$ & 0.04883 & 0.0121 & -0.01332 & -0.70981 \\
$\mathrm{C}$ & 0.02021 & -0.0005 & 1.41582 & 0.43585 \\
$\mathrm{C}$ & 1.39989 & -0.00471 & 2.07929 & -0.37502 \\
$\mathrm{C}$ & 1.06723 & 0.28389 & 3.54576 & 0.12247 \\
$\mathrm{C}$ & -0.04325 & 1.3621 & 3.48955 & -0.02663 \\
$\mathrm{C}$ & -0.77078 & 1.16527 & 2.10186 & 0.2388 \\
$\mathrm{C}$ & -0.6833 & 2.45003 & 1.26056 & -0.35231 \\
$\mathrm{C}$ & -2.24258 & 0.77119 & 2.32073 & -0.02242 \\
$\mathrm{C}$ & -3.02893 & 1.74555 & 3.21893 & -0.04844 \\
$\mathrm{C}$ & -2.19706 & 2.29923 & 4.41389 & 0.23056 \\
$\mathrm{C}$ & -3.03682 & 2.59354 & 5.65075 & -0.34997 \\
$\mathrm{C}$ & -4.28218 & 3.24527 & 5.53244 & -0.0108 \\
$\mathrm{C}$ & -5.05333 & 3.60151 & 6.62658 & -0.51027 \\
$\mathrm{C}$ & -0.99825 & 1.37092 & 4.69583 & 0.04208 \\
$\mathrm{C}$ & -0.30212 & 1.77913 & 5.99718 & -0.07501 \\
$\mathrm{C}$ & -1.25462 & 1.62917 & 7.18442 & 0.02157 \\
$\mathrm{C}$ & -2.58575 & 2.32847 & 6.96122 & 0.17201 \\
$\mathrm{C}$ & -3.35124 & 2.68446 & 8.06997 & -0.53202 \\
$\mathrm{C}$ & -4.63464 & 3.33601 & 7.98515 & 0.75933 \\
$\mathrm{O}$ & -5.32805 & 3.65268 & 9.00102 & -0.82494 \\
$\mathrm{H}$ & 0.46141 & 0.83509 & -0.29709 & 0.37772 \\
$\mathrm{H}$ & -0.48326 & -0.9425 & 1.65551 & 0.01289 \\
$\mathrm{H}$ & 1.93682 & -0.9433 & 1.91609 & 0.07261 \\
$\mathrm{H}$ & 2.01365 & 0.80336 & 1.65842 & 0.06573 \\
$\mathrm{H}$ & 0.68279 & -0.62535 & 4.0246 & -0.04193 \\
$\mathrm{H}$ & 1.93498 & 0.60562 & 4.12584 & -0.00845 \\
$\mathrm{H}$ & 0.44734 & 2.34433 & 3.46794 & -0.01749 \\
$\mathrm{H}$ & -1.20288 & 2.3395 & 0.30335 & 0.06274 \\
$\mathrm{H}$ & -1.13929 & 3.28702 & 1.79337 & 0.07455 \\
$\mathrm{H}$ & 0.35801 & 2.73313 & 1.06033 & 0.0474 \\
$\mathrm{H}$ & -2.73919 & 0.65812 & 1.34911 & -0.02005 \\
$\mathrm{H}$ & -2.25963 & -0.22349 & 2.78074 & -0.04855 \\
$\mathrm{H}$ & -3.41475 & 2.57823 & 2.62264 & 0.00963 \\
$\mathrm{H}$ & -3.90827 & 1.22642 & 3.61086 & 0.00233 \\
$\mathrm{H}$ & -1.74775 & 3.24987 & 4.07204 & -0.04135 \\
$\mathrm{H}$ & -4.65788 & 3.48835 & 4.53877 & 0.05393 \\
$\mathrm{H}$ & -6.009 & 4.10159 & 6.48661 & 0.12489 \\
$\mathrm{H}$ & -1.38048 & 0.34858 & 4.84231 & 0.00823 \\
$\mathrm{H}$ & 0.60021 & 1.17566 & 6.15889 & -0.0223 \\
$\mathrm{H}$ & 0.02498 & 2.82511 & 5.91218 & 0.00443 \\
$\mathrm{H}$ & -1.42348 & 0.55669 & 7.36411 & -0.01613 \\
$\mathrm{H}$ & -0.78717 & 2.01686 & 8.09633 & 0.00054 \\
$\mathrm{H}$ & -2.97814 & 2.46654 & 9.06965 & 0.11362 \\
\hline & & & &
\end{tabular}




\begin{tabular}{|c|c|c|c|c|c|}
\hline bisphenol A & Atom & & Coordinates & & Partial Charges \\
\hline & $\mathrm{C}$ & 0.06363 & 0.01932 & 0.04499 & -0.32861 \\
\hline & $\mathrm{C}$ & 0.02513 & -0.00237 & 1.59253 & 0.44587 \\
\hline & $\mathrm{C}$ & 1.49503 & 0.02355 & 2.07974 & -0.28817 \\
\hline & $\mathrm{C}$ & -0.66097 & -1.26344 & 2.13768 & 0.0274 \\
\hline & $\mathrm{C}$ & -0.85368 & -1.42056 & 3.52668 & -0.17188 \\
\hline & $\mathrm{C}$ & -1.43697 & -2.54946 & 4.07914 & -0.43208 \\
\hline & $\mathrm{C}$ & -1.89724 & -3.66433 & 3.27861 & 0.70566 \\
\hline & $\mathrm{C}$ & -1.69266 & -3.47224 & 1.86297 & -0.39056 \\
\hline & $\mathrm{C}$ & -1.10419 & -2.32845 & 1.333 & -0.2722 \\
\hline & $\mathrm{O}$ & -2.42975 & -4.70344 & 3.77598 & -0.81462 \\
\hline & $\mathrm{C}$ & -0.73907 & 1.26477 & 2.03276 & 0.05477 \\
\hline & $\mathrm{C}$ & -2.14195 & 1.29103 & 1.9498 & -0.20249 \\
\hline & $\mathrm{C}$ & -2.88173 & 2.42279 & 2.27544 & -0.23879 \\
\hline & $\mathrm{C}$ & -2.22727 & 3.57516 & 2.71443 & 0.42191 \\
\hline & $\mathrm{C}$ & -0.84037 & 3.57804 & 2.8187 & -0.40027 \\
\hline & $\mathrm{C}$ & -0.11125 & 2.43273 & 2.47586 & -0.15015 \\
\hline & $\mathrm{O}$ & -2.99964 & 4.67533 & 3.03757 & -0.60259 \\
\hline & $\mathrm{H}$ & 0.54567 & 0.93865 & -0.30321 & 0.04047 \\
\hline & $\mathrm{H}$ & 0.63089 & -0.83177 & -0.34303 & 0.06067 \\
\hline & $\mathrm{H}$ & -0.94079 & -0.01724 & -0.38336 & 0.0446 \\
\hline & $\mathrm{H}$ & 1.55347 & 0.0863 & 3.16889 & 0.02942 \\
\hline & $\mathrm{H}$ & 1.9827 & -0.9059 & 1.77643 & 0.04747 \\
\hline & $\mathrm{H}$ & 2.06454 & 0.85816 & 1.65156 & 0.02636 \\
\hline & $\mathrm{H}$ & -0.54491 & -0.61924 & 4.19796 & 0.09518 \\
\hline & $\mathrm{H}$ & -1.5691 & -2.62429 & 5.15613 & 0.11752 \\
\hline & $\mathrm{H}$ & -2.02111 & -4.26996 & 1.20057 & 0.10107 \\
\hline & $\mathrm{H}$ & -0.99117 & -2.27596 & 0.25304 & 0.12381 \\
\hline & $\mathrm{H}$ & -2.66078 & 0.38801 & 1.647 & 0.13939 \\
\hline & $\mathrm{H}$ & -3.96454 & 2.41908 & 2.21062 & 0.15299 \\
\hline & $\mathrm{H}$ & -0.32117 & 4.46737 & 3.17079 & 0.14619 \\
\hline & $\mathrm{H}$ & 0.96736 & 2.47124 & 2.5709 & 0.12542 \\
\hline & $\mathrm{H}$ & -2.41675 & 5.37531 & 3.34904 & 0.38623 \\
\hline
\end{tabular}




\begin{tabular}{|c|c|c|c|c|c|}
\hline \multirow{46}{*}{ 17- $\alpha$ - Ethynylestradiol } & \multirow{3}{*}{$\begin{array}{l}\mathrm{O} \\
\mathrm{C}\end{array}$} & \multirow{2}{*}{\multicolumn{2}{|c|}{$\begin{array}{ll}-6.31537 & -0.18184\end{array}$}} & \multicolumn{2}{|r|}{050280} \\
\hline & & & & -0.10129 & -0.59389 \\
\hline & & -4.94435 & -0.28703 & -0.03928 & 0.43473 \\
\hline & $\mathrm{C}$ & -4.19663 & 0.88466 & -0.05573 & -0.35196 \\
\hline & $\mathrm{C}$ & -2.80173 & 0.85445 & 0.06373 & 0.15109 \\
\hline & $\mathrm{C}$ & -2.12829 & -0.3771 & 0.20345 & -0.10616 \\
\hline & $\mathrm{C}$ & -2.90867 & -1.54159 & 0.22159 & -0.05672 \\
\hline & $\mathrm{C}$ & -4.29678 & -1.51284 & 0.10194 & -0.43044 \\
\hline & $\mathrm{C}$ & -0.61003 & -0.43838 & 0.38229 & 0.02366 \\
\hline & $\mathrm{C}$ & 0.04981 & -1.70492 & -0.20717 & 0.03103 \\
\hline & $\mathrm{C}$ & 1.57611 & -1.7413 & 0.00901 & -0.27009 \\
\hline & $\mathrm{C}$ & 2.26078 & -0.46364 & -0.48605 & 0.91017 \\
\hline & $\mathrm{C}$ & 1.576 & 0.7526 & 0.17922 & 0.01049 \\
\hline & $\mathrm{C}$ & 2.50637 & 1.94114 & -0.13682 & -0.22884 \\
\hline & $\mathrm{C}$ & 3.92548 & 1.31021 & -0.21393 & 0.07504 \\
\hline & $\mathrm{C}$ & 3.75045 & -0.23694 & 0.01525 & -0.21566 \\
\hline & $\mathrm{C}$ & 3.91077 & -0.58534 & 1.42084 & 0.49672 \\
\hline & $\mathrm{C}$ & 3.9238 & -0.79025 & 2.65084 & -1.09294 \\
\hline & $\mathrm{O}$ & 4.7174 & -0.9886 & -0.77094 & -0.71367 \\
\hline & $\mathrm{C}$ & 0.08243 & 0.85051 & -0.12667 & 0.07903 \\
\hline & $\mathrm{C}$ & -0.6018 & 2.06817 & 0.50009 & 0.04711 \\
\hline & $\mathrm{C}$ & -2.0504 & 2.17318 & 0.02108 & -0.1237 \\
\hline & $\mathrm{C}$ & 2.21129 & -0.41162 & -2.02854 & -0.64517 \\
\hline & $\mathrm{H}$ & -0.41767 & -0.46814 & 1.46607 & 0.01222 \\
\hline & $\mathrm{H}$ & 1.67255 & 0.56862 & 1.25637 & 0.00821 \\
\hline & $\mathrm{H}$ & -0.06806 & 0.91783 & -1.21589 & -0.04855 \\
\hline & $\mathrm{H}$ & -6.69499 & -1.06558 & -0.11813 & 0.38978 \\
\hline & $\mathrm{H}$ & -4.71419 & 1.83358 & -0.16028 & 0.16244 \\
\hline & $\mathrm{H}$ & -2.42669 & -2.5048 & 0.33658 & 0.10935 \\
\hline & $\mathrm{H}$ & -4.86732 & -2.4386 & 0.12138 & 0.15244 \\
\hline & $\mathrm{H}$ & -0.37825 & -2.59809 & 0.25859 & 0.00509 \\
\hline & $\mathrm{H}$ & -0.20035 & -1.77122 & -1.27447 & -0.04177 \\
\hline & $\mathrm{H}$ & 1.80489 & -1.84865 & 1.07407 & 0.04333 \\
\hline & $\mathrm{H}$ & 1.99513 & -2.62131 & -0.49305 & 0.00619 \\
\hline & $\mathrm{H}$ & 2.23513 & 2.41593 & -1.08804 & 0.01384 \\
\hline & $\mathrm{H}$ & 2.44595 & 2.71542 & 0.632 & 0.03501 \\
\hline & $\mathrm{H}$ & 4.37796 & 1.49493 & -1.19665 & -0.03201 \\
\hline & $\mathrm{H}$ & 4.60538 & 1.70863 & 0.53731 & -0.00291 \\
\hline & $\mathrm{H}$ & 4.70365 & -0.63194 & -1.66631 & 0.40495 \\
\hline & $\mathrm{H}$ & -0.56812 & 1.97709 & 1.59322 & -0.01649 \\
\hline & $\mathrm{H}$ & -0.06473 & 2.98724 & 0.24549 & -0.01475 \\
\hline & $\mathrm{H}$ & -2.058 & 2.53974 & -1.01476 & 0.03362 \\
\hline & $\mathrm{H}$ & -2.6008 & 2.91919 & 0.60571 & 0.02818 \\
\hline & $\mathrm{H}$ & 2.73125 & -1.27978 & -2.44488 & 0.0985 \\
\hline & $\mathrm{H}$ & 1.18711 & -0.44036 & -2.40875 & 0.1125 \\
\hline & $\mathrm{H}$ & 2.67632 & 0.48995 & -2.43854 & 0.11098 \\
\hline
\end{tabular}




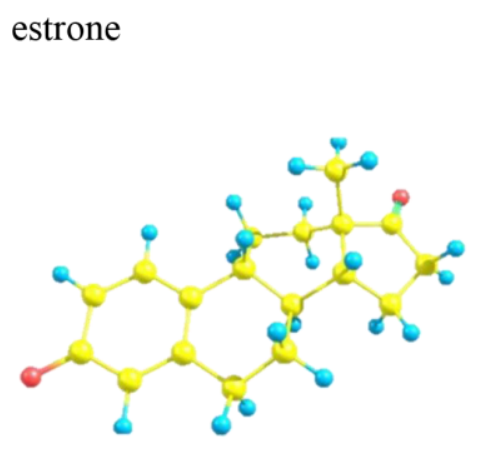

\begin{tabular}{lrrrr} 
Atom & \multicolumn{3}{c}{ Coordinates } & \multicolumn{1}{c}{ Partial Charges } \\
\hline $\mathrm{O}$ & -0.02394 & -0.08503 & -0.00103 & -0.54838 \\
$\mathrm{C}$ & -0.00862 & -0.03456 & 1.21123 & 0.46002 \\
$\mathrm{C}$ & 1.25924 & -0.01154 & 2.08071 & 0.39826 \\
$\mathrm{C}$ & 2.13211 & 1.20697 & 1.70867 & -0.12628 \\
$\mathrm{C}$ & 3.35511 & 1.36362 & 2.63859 & 0.04215 \\
$\mathrm{C}$ & 3.08468 & 0.94533 & 4.11289 & 0.09068 \\
$\mathrm{C}$ & 3.97426 & 1.66656 & 5.11795 & -0.2525 \\
$\mathrm{C}$ & 5.35398 & 1.8247 & 4.86946 & -0.06731 \\
$\mathrm{C}$ & 6.22051 & 2.41155 & 5.77646 & -0.48002 \\
$\mathrm{C}$ & 5.77523 & 2.90492 & 7.06118 & 0.76769 \\
$\mathrm{O}$ & 6.55306 & 3.44841 & 7.90457 & -0.82778 \\
$\mathrm{C}$ & 4.36469 & 2.71222 & 7.28912 & -0.55554 \\
$\mathrm{C}$ & 3.50181 & 2.12363 & 6.36682 & 0.1763 \\
$\mathrm{C}$ & 2.02916 & 1.99699 & 6.72313 & -0.00753 \\
$\mathrm{C}$ & 1.31895 & 0.90924 & 5.91655 & -0.09923 \\
$\mathrm{C}$ & 1.58375 & 1.09493 & 4.42005 & -0.01619 \\
$\mathrm{C}$ & 0.73755 & 0.14396 & 3.54777 & 0.10156 \\
$\mathrm{C}$ & -0.74269 & 0.59471 & 3.41994 & -0.03949 \\
$\mathrm{C}$ & -1.24414 & 0.0001 & 2.10113 & -0.29271 \\
$\mathrm{C}$ & 2.01312 & -1.33166 & 1.82442 & -0.34293 \\
$\mathrm{H}$ & 2.43996 & 1.12583 & 0.66181 & -0.00129 \\
$\mathrm{H}$ & 1.51217 & 2.10909 & 1.77141 & -0.00398 \\
$\mathrm{H}$ & 4.19594 & 0.78724 & 2.24227 & -0.00338 \\
$\mathrm{H}$ & 3.67363 & 2.4099 & 2.62399 & -0.01412 \\
$\mathrm{H}$ & 3.29523 & -0.13649 & 4.18392 & -0.01351 \\
$\mathrm{H}$ & 5.76492 & 1.46634 & 3.92591 & 0.06935 \\
$\mathrm{H}$ & 7.27713 & 2.51435 & 5.54042 & 0.11656 \\
$\mathrm{H}$ & 3.9721 & 3.05576 & 8.24503 & 0.12084 \\
$\mathrm{H}$ & 1.92909 & 1.79934 & 7.79603 & 0.01217 \\
$\mathrm{H}$ & 1.51948 & 2.95553 & 6.54385 & -0.00496 \\
$\mathrm{H}$ & 1.68666 & -0.0807 & 6.22081 & 0.02165 \\
$\mathrm{H}$ & 0.24081 & 0.92563 & 6.12375 & -0.00678 \\
$\mathrm{H}$ & 1.29792 & 2.12826 & 4.16857 & 0.02054 \\
$\mathrm{H}$ & 0.75385 & -0.84864 & 4.01801 & -0.0599 \\
$\mathrm{H}$ & -1.34276 & 0.29076 & 4.27982 & 0.02333 \\
$\mathrm{H}$ & -0.78741 & 1.68838 & 3.36962 & 0.00463 \\
$\mathrm{H}$ & -2.06338 & 0.53665 & 1.61823 & 0.0876 \\
$\mathrm{H}$ & -1.56969 & -1.04075 & 2.23342 & 0.07626 \\
& 2.27349 & -1.41618 & 0.76587 & 0.06878 \\
$\mathrm{H}$ & 2.92859 & -1.38397 & 2.41581 & 0.06163 \\
& 1.39516 & -2.1956 & 2.09224 & 0.04378 \\
\hline & & & &
\end{tabular}


(E)-diethylstilbestrol

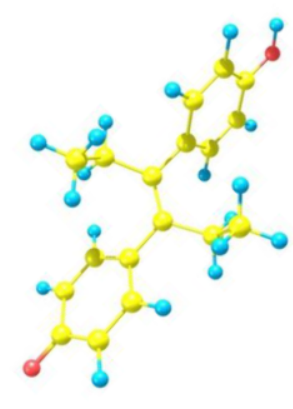

\begin{tabular}{lrrrr} 
Atom & \multicolumn{3}{c}{ Coordinates } & Partial Charges \\
\hline $\mathrm{O}$ & 0.35169 & 0.11722 & 0.00505 & -0.79451 \\
$\mathrm{C}$ & 0.32226 & 0.10512 & 1.26863 & 0.77529 \\
$\mathrm{C}$ & 1.06025 & 1.04425 & 2.08879 & -0.47607 \\
$\mathrm{C}$ & 1.0055 & 1.0325 & 3.46875 & -0.04206 \\
$\mathrm{C}$ & 0.23684 & 0.09375 & 4.20139 & -0.09809 \\
$\mathrm{C}$ & 0.19769 & 0.07428 & 5.67122 & -0.03201 \\
$\mathrm{C}$ & 0.11077 & 1.19439 & 6.45871 & -0.33393 \\
$\mathrm{C}$ & 0.29771 & 1.14449 & 7.93657 & 0.20651 \\
$\mathrm{C}$ & 1.49658 & 0.6923 & 8.52076 & -0.25161 \\
$\mathrm{C}$ & 1.69862 & 0.68211 & 9.89952 & -0.2115 \\
$\mathrm{C}$ & 0.69519 & 1.14563 & 10.74921 & 0.42217 \\
$\mathrm{O}$ & 0.93416 & 1.12228 & 12.11084 & -0.61866 \\
$\mathrm{C}$ & -0.49814 & 1.62081 & 10.20655 & -0.51349 \\
$\mathrm{C}$ & -0.68534 & 1.61933 & 8.82379 & 0.05882 \\
$\mathrm{C}$ & -0.27337 & 2.57724 & 5.92383 & 0.33861 \\
$\mathrm{C}$ & -1.68138 & 2.64623 & 5.30687 & -0.16906 \\
$\mathrm{C}$ & 0.18003 & -1.31596 & 6.31485 & 0.11831 \\
$\mathrm{C}$ & -1.20429 & -1.74644 & 6.83327 & -0.01346 \\
$\mathrm{C}$ & -0.45436 & -0.86677 & 3.41723 & -0.0674 \\
$\mathrm{C}$ & -0.43531 & -0.86211 & 2.03571 & -0.47209 \\
$\mathrm{H}$ & 1.69287 & 1.76166 & 1.57178 & 0.11777 \\
$\mathrm{H}$ & 1.62139 & 1.74198 & 4.01442 & 0.0818 \\
$\mathrm{H}$ & 2.29684 & 0.35496 & 7.87049 & 0.13305 \\
$\mathrm{H}$ & 2.63423 & 0.33314 & 10.32375 & 0.14521 \\
$\mathrm{H}$ & 0.15897 & 1.47146 & 12.56242 & 0.40582 \\
$\mathrm{H}$ & -1.28618 & 1.98714 & 10.86167 & 0.16107 \\
$\mathrm{H}$ & -1.62588 & 1.98701 & 8.42668 & 0.09097 \\
$\mathrm{H}$ & -0.21295 & 3.29389 & 6.74966 & -0.11902 \\
$\mathrm{H}$ & 0.44396 & 2.93117 & 5.18079 & -0.03263 \\
$\mathrm{H}$ & -1.90217 & 3.66098 & 4.95585 & -0.00267 \\
$\mathrm{H}$ & -1.76464 & 1.96763 & 4.4562 & 0.06269 \\
$\mathrm{H}$ & -2.44812 & 2.36711 & 6.03789 & 0.00441 \\
$\mathrm{H}$ & 0.53189 & -2.04383 & 5.58023 & -0.04081 \\
$\mathrm{H}$ & 0.88375 & -1.36139 & 7.14832 & -0.00181 \\
$\mathrm{H}$ & -1.16952 & -2.76507 & 7.23759 & -0.02402 \\
$\mathrm{H}$ & -1.55025 & -1.08088 & 7.62856 & -0.02695 \\
$\mathrm{H}$ & -1.95132 & -1.7259 & 6.03506 & -0.0091 \\
$\mathrm{H}$ & -1.05118 & -1.62765 & 3.91534 & 0.11356 \\
$\mathrm{H}$ & -0.99735 & -1.60633 & 1.47685 & 0.11491 \\
\hline & & & &
\end{tabular}


(Z)-diethylstilbestrol

\begin{tabular}{lrrrr} 
Atom & \multicolumn{3}{c}{ Coordinates } & Partial Charges \\
\hline $\mathrm{O}$ & -3.88502 & -3.2652 & -0.56398 & -0.78584 \\
$\mathrm{C}$ & -3.19784 & -2.21287 & -0.4369 & 0.74015 \\
$\mathrm{C}$ & -1.97769 & -1.96663 & -1.1808 & -0.38152 \\
$\mathrm{C}$ & -1.22621 & -0.82173 & -1.02247 & -0.11381 \\
$\mathrm{C}$ & -1.59057 & 0.21965 & -0.12925 & -0.09566 \\
$\mathrm{C}$ & -0.79002 & 1.43687 & 0.02821 & 0.04389 \\
$\mathrm{C}$ & 0.58264 & 1.50073 & -0.05229 & -0.47061 \\
$\mathrm{C}$ & 1.47904 & 0.32143 & 0.05923 & 0.41532 \\
$\mathrm{C}$ & 2.70728 & 0.24279 & -0.62706 & -0.31918 \\
$\mathrm{C}$ & 3.59325 & -0.82466 & -0.46256 & -0.25034 \\
$\mathrm{C}$ & 3.27521 & -1.85558 & 0.41394 & 0.41119 \\
$\mathrm{O}$ & 4.17016 & -2.90178 & 0.55197 & -0.6106 \\
$\mathrm{C}$ & 2.07373 & -1.80183 & 1.12612 & -0.33843 \\
$\mathrm{C}$ & 1.19927 & -0.73701 & 0.94855 & -0.2648 \\
$\mathrm{C}$ & 1.29835 & 2.83894 & -0.22171 & 0.2861 \\
$\mathrm{C}$ & 1.34015 & 3.36324 & -1.67135 & -0.17192 \\
$\mathrm{C}$ & -1.58421 & 2.72319 & 0.27353 & 0.12693 \\
$\mathrm{C}$ & -1.58146 & 3.19444 & 1.74009 & -0.11635 \\
$\mathrm{C}$ & -2.8083 & 0.01967 & 0.57391 & -0.13293 \\
$\mathrm{C}$ & -3.56599 & -1.12858 & 0.4505 & -0.436 \\
$\mathrm{H}$ & -1.67704 & -2.72406 & -1.90033 & 0.09828 \\
$\mathrm{H}$ & -0.33121 & -0.69787 & -1.62484 & 0.12541 \\
$\mathrm{H}$ & 2.98332 & 1.02291 & -1.32736 & 0.16927 \\
$\mathrm{H}$ & 4.52426 & -0.86489 & -1.01839 & 0.14418 \\
$\mathrm{H}$ & 3.77027 & -3.56603 & 1.12278 & 0.38715 \\
$\mathrm{H}$ & 1.81739 & -2.59806 & 1.82176 & 0.14722 \\
$\mathrm{H}$ & 0.27057 & -0.72413 & 1.50462 & 0.19181 \\
$\mathrm{H}$ & 0.83598 & 3.60694 & 0.40463 & -0.02513 \\
$\mathrm{H}$ & 2.3252 & 2.74571 & 0.14664 & -0.06949 \\
$\mathrm{H}$ & 1.8718 & 4.3212 & -1.73116 & -0.00207 \\
$\mathrm{H}$ & 1.84256 & 2.65801 & -2.33917 & 0.02394 \\
$\mathrm{H}$ & 0.33029 & 3.51108 & -2.0642 & 0.03609 \\
$\mathrm{H}$ & -1.205 & 3.53227 & -0.35728 & -0.00316 \\
$\mathrm{H}$ & -2.6168 & 2.5629 & -0.04347 & -0.02995 \\
$\mathrm{H}$ & -2.1707 & 4.11159 & 1.85857 & 0.00113 \\
$\mathrm{H}$ & -2.00963 & 2.43301 & 2.3972 & 0.00981 \\
$\mathrm{H}$ & -0.56717 & 3.39668 & 2.09598 & 0.0148 \\
$\mathrm{H}$ & -3.15376 & 0.78356 & 1.26679 & 0.13667 \\
$\mathrm{H}$ & -4.48129 & -1.24729 & 1.02504 & 0.10846 \\
\hline & & & & \\
& & &
\end{tabular}




\section{Appendix 7.1}

Histogram showing the number of peaks identified per nominal mass by a) FT-ICR MS, b) SA-TIMS-FT-ICR MS, and c) OSA-TIMS-FT-ICR MS.

a)

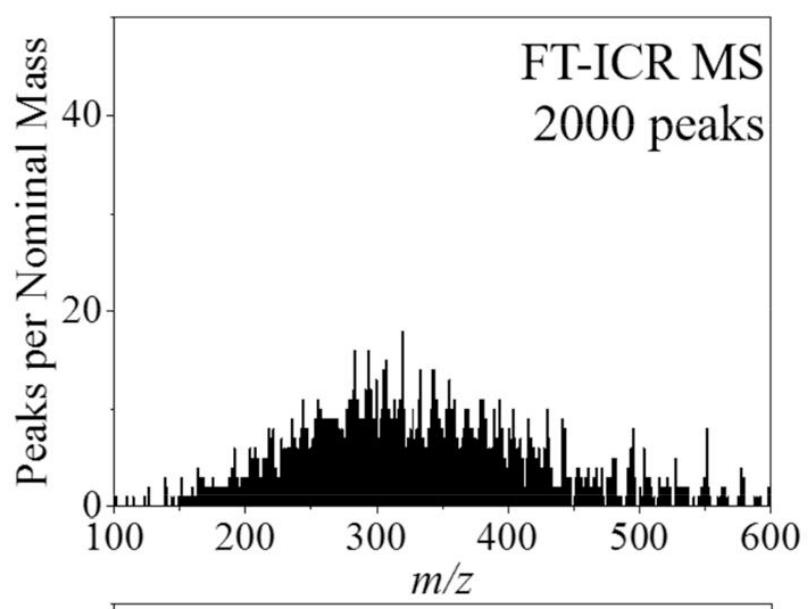

b)

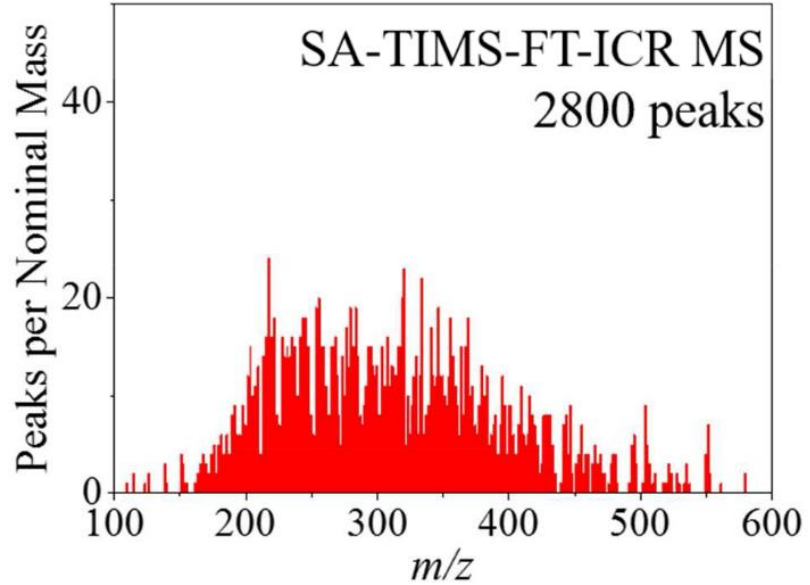

c)

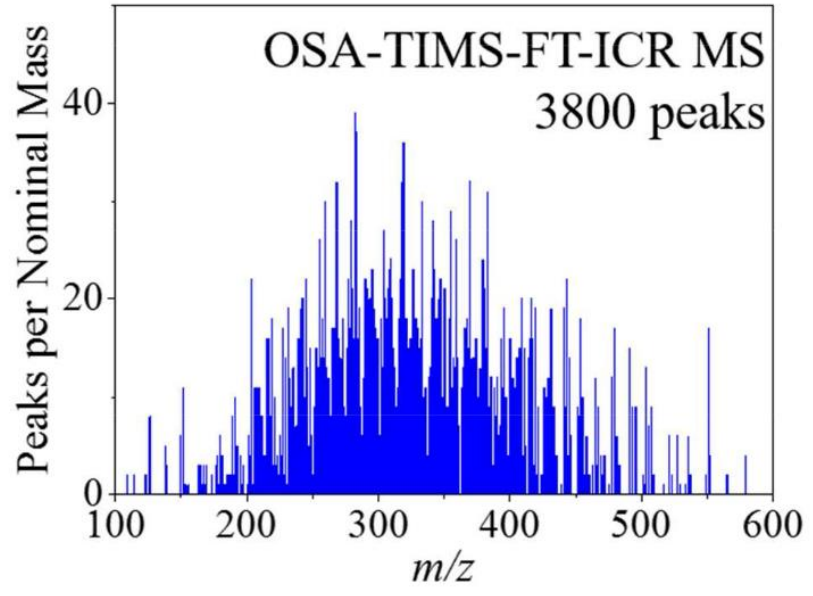




\section{Appendix 7.2}

a) Typical 2D-IMS-MS contour plot for the APPI-OSA-TIMS-FT-ICR MS of coal tar standard reference material, with two regions of interest highlighted. The mass spectrums are also shown for b) the 8Mword FT-ICR MS spectrum, c) region 1 of the OSA-TIMSFT-ICR MS analysis, and d) region 2 of the OSA-TIMS-FT-ICR MS analysis.

a)

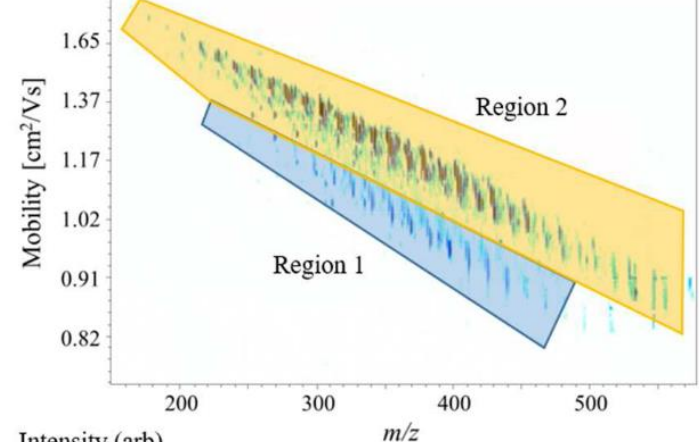

b)

Intensity (arb) $m / z$

c)
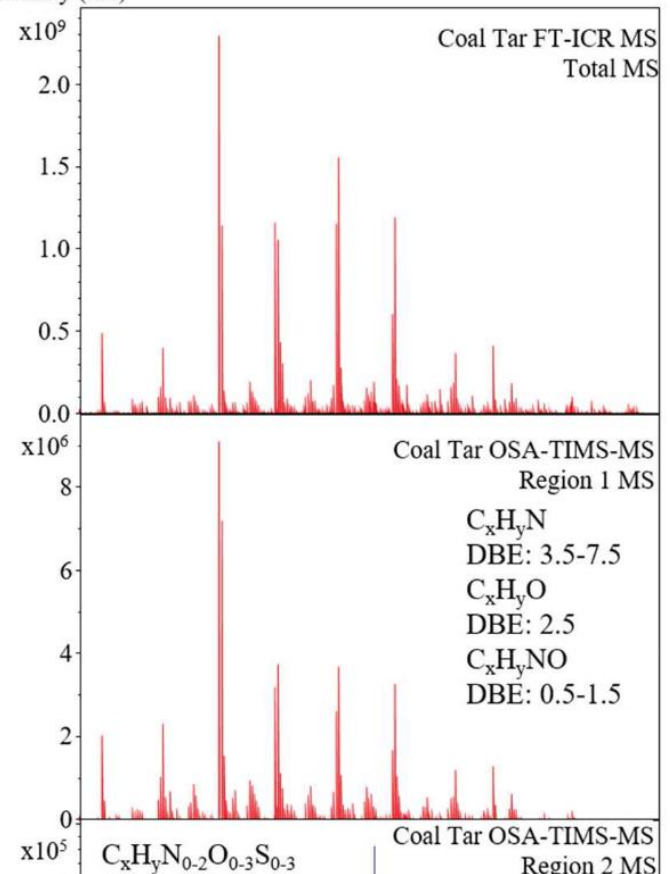

d)

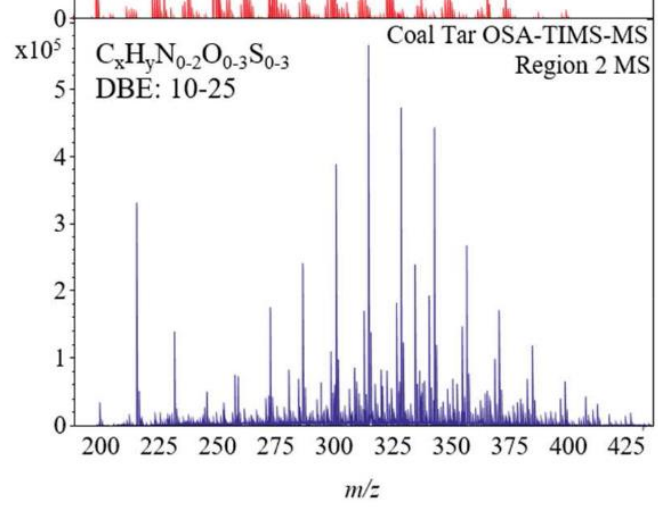




\section{Appendix 8.1}

Schematic of the TIMS

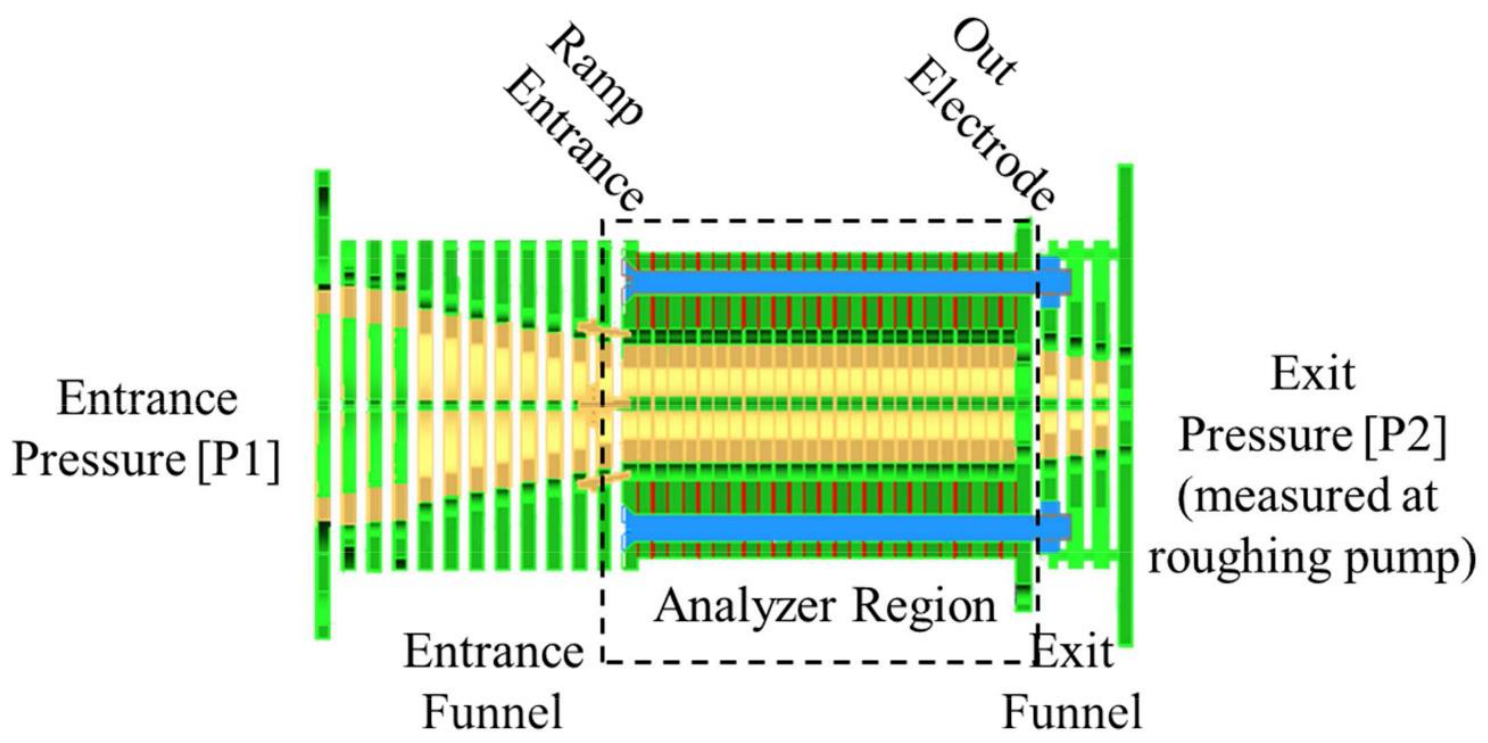




\section{Appendix 8.2}

Carbon number vs DBE plot for the $\mathrm{O}_{0-3}$ chemical classes for the three different experimental time points.

$\mathrm{t}-\mathrm{Oh}$
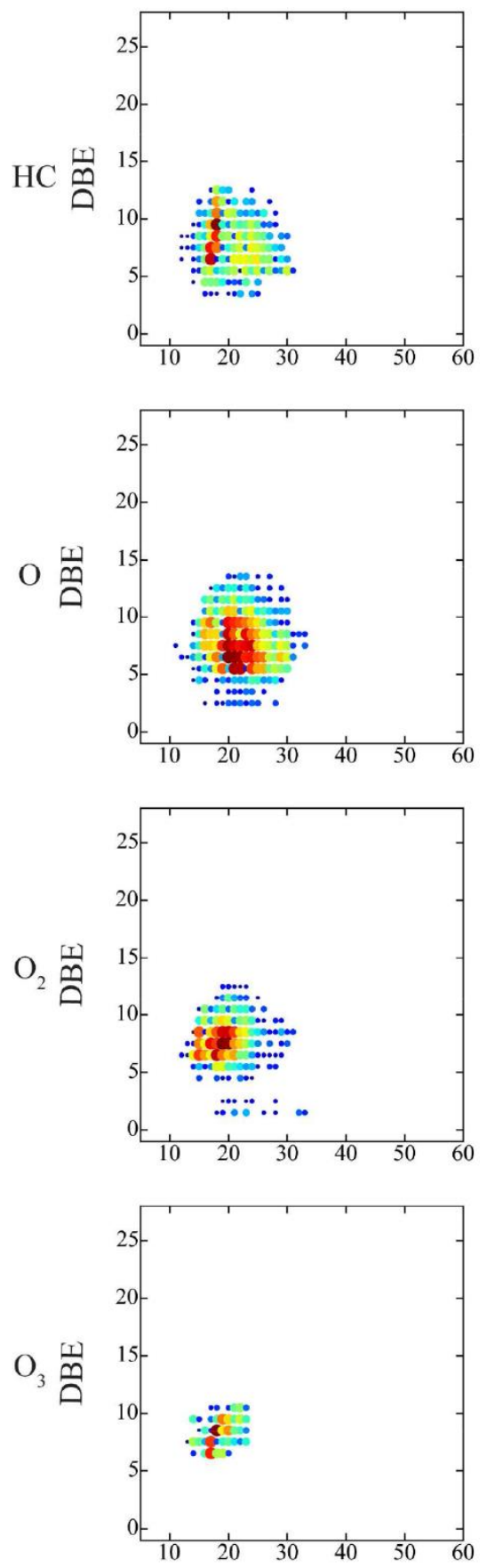

$\mathrm{t}-16 \mathrm{~h}$
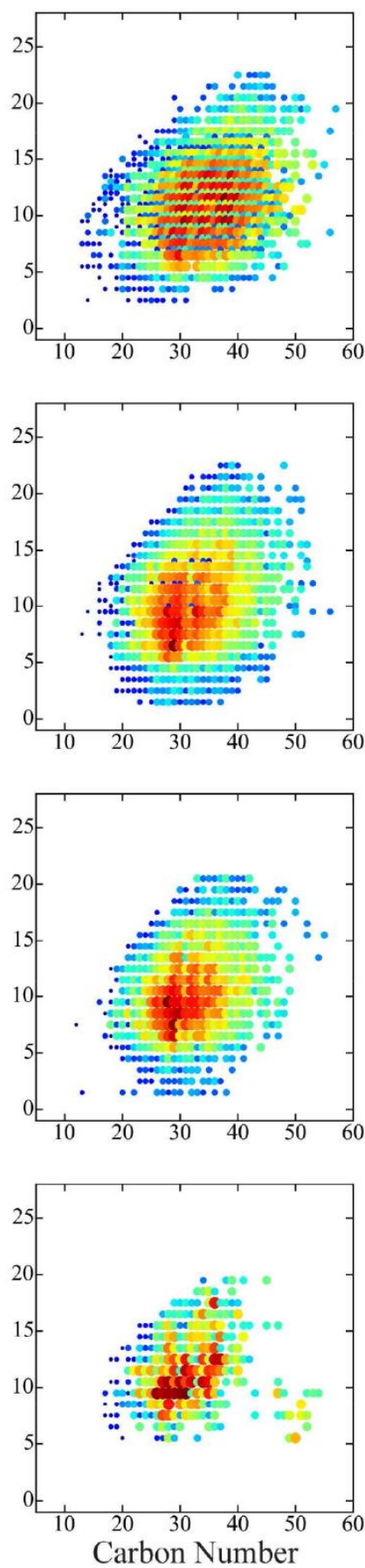

$\mathrm{t}-115 \mathrm{~h}$
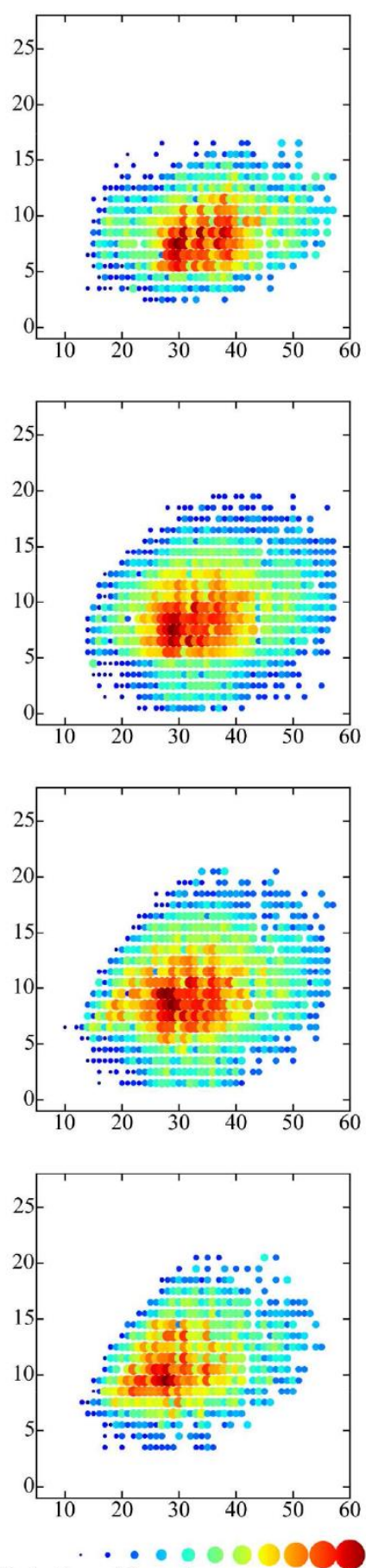

Relative Abundance $\longrightarrow 100 \%$ 


\section{Appendix 8.3}

Carbon number vs DBE plot for the $\mathrm{O}_{4-5}$ chemical classes for the three different experimental time points.

\section{$\mathrm{t}-115 \mathrm{~h}$}
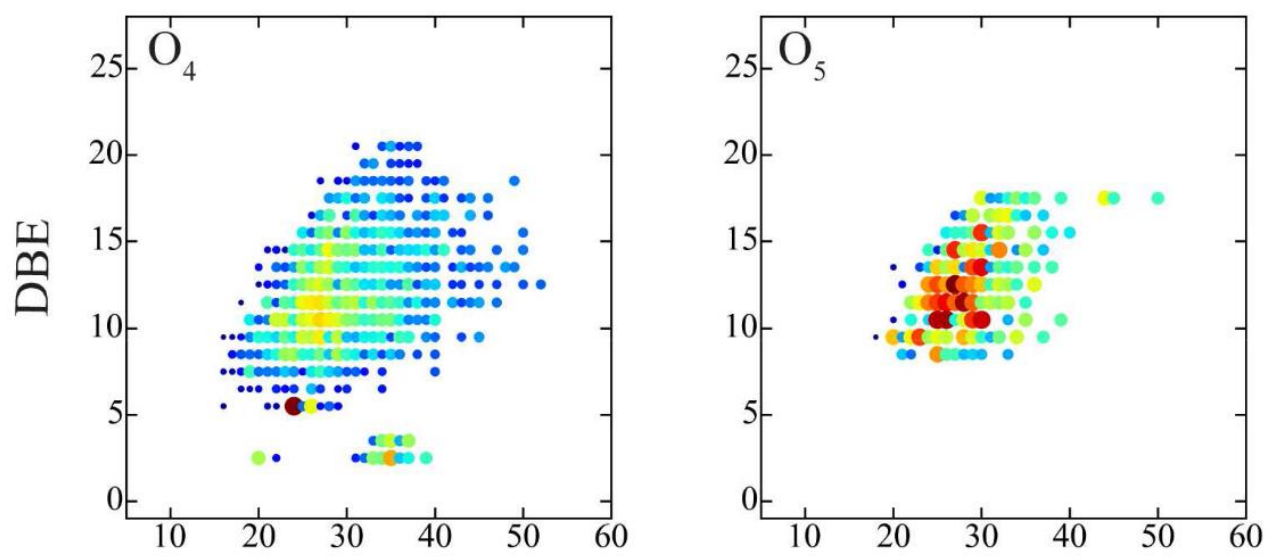

Carbon Number

Relative Abundance $\longrightarrow 100 \%$ 


\section{Appendix 8.4}

Carbon number vs DBE plot for the $\mathrm{N}_{1} \mathrm{O}_{0-3}$ chemical classes for the three different experimental time points.

$-0 h$
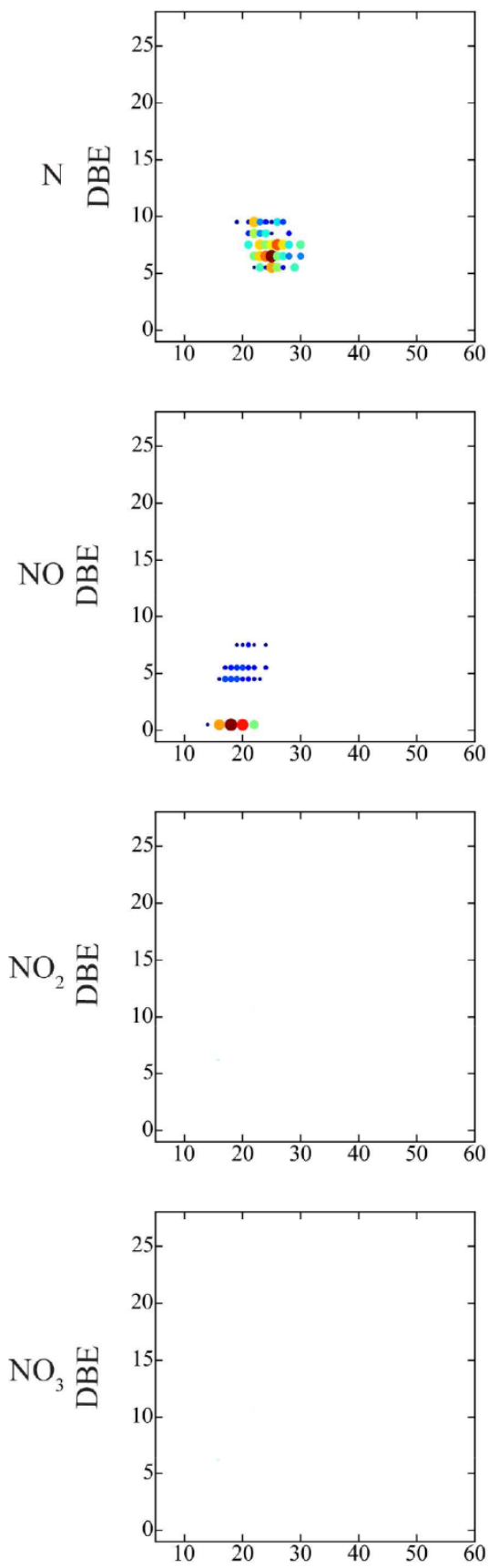

$\mathrm{t}-16 \mathrm{~h}$
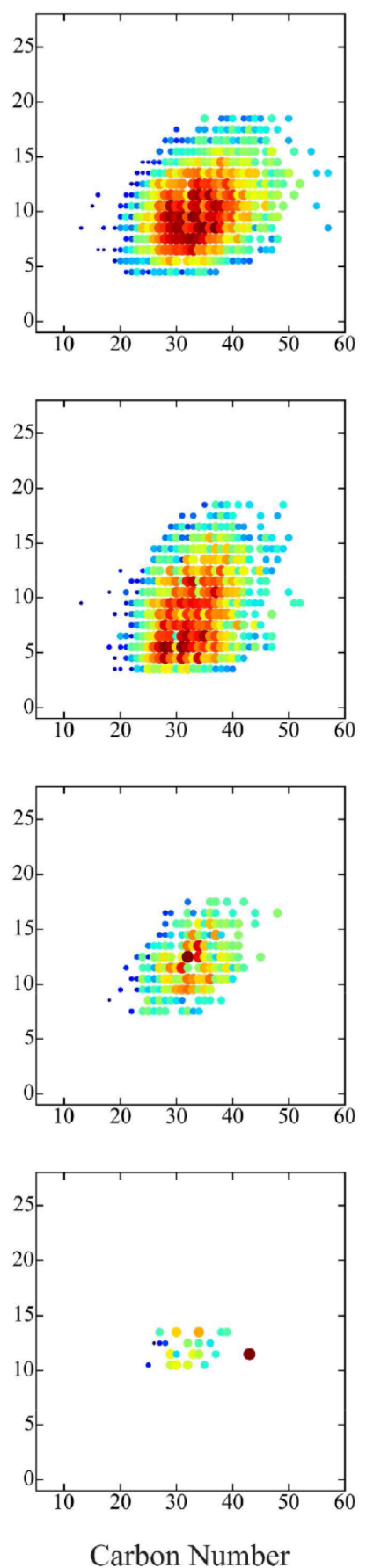

$\mathrm{t}-115 \mathrm{~h}$
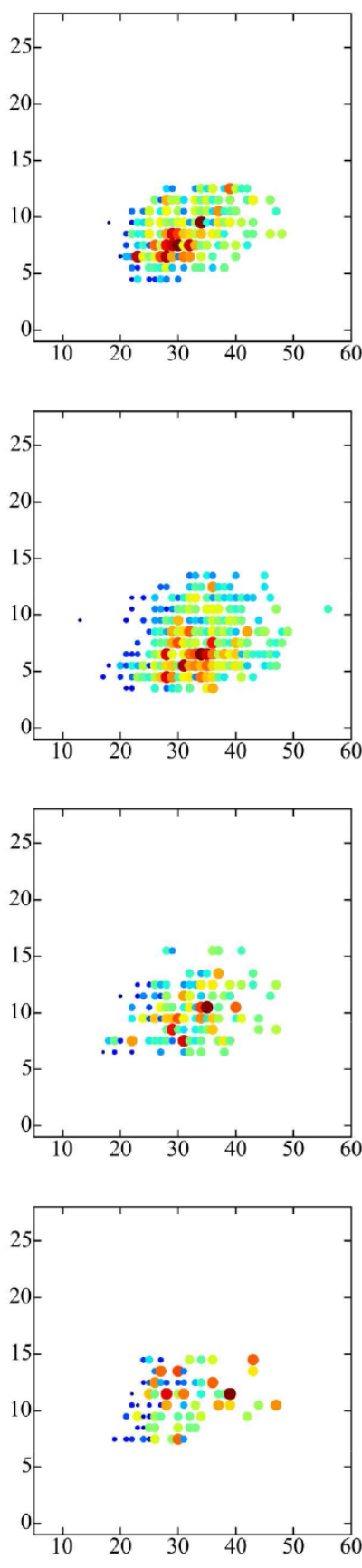

Relative Abundance $\rightarrow 100 \%$ 
VITAE 
VITAE

\section{PAOLO BENIGNI}

Born: Rome, Italy

2013

B.S. Chemistry

Florida International University

Miami, Florida

$2014-2017$

Doctoral Candidate

Florida International University

Miami, Florida

Graduate Research Assistant

Florida International University

Miami, Florida

\section{SELECTED PEER-REVIEWED PUBLICATIONS}

- P. Benigni, K. Sandoval, C. J. Thompson, Gardinali, F. Fernandez-Lima, Analysis of photo-irradiated water accommodated fractions of crude oils using tandem TIMS and FT-ICR MS, Env. Sci. Tech, 2017, 51 (11), 5978-5988

- P. Benigni, C. Bravo, J. M. E. Quirke, J. D. DeBord, A. M. Mebel, and F. Fernandez-Lima, Analysis of geologically relevant metal-porphyrins using Trapped Ion Mobility Spectrometry-Mass Spectrometry and theoretical calculations, Energy Fuels, 2016, 30 (12), 10341-10347

- P. Benigni, R. Marin, K. Sandoval, P. Gardinali, F. Fernandez-Lima, Chemical Analysis of Water Accommodated Fractions of Crude Oil Spills using TIMS FT-ICR MS, J. Vis. Exp 2017, (121), e55352.

- $\quad$ P. Benigni, F. Fernandez-Lima, Oversampling Trapped Ion Mobility Spectrometry coupled to FT-ICR MS, Anal. Chem. 2016, 88 (14), 7404-12

- $\quad$ C. E Ramirez, M. Nouzova, P. Benigni, J. M. Quirke, F. G Noriega, F. FernandezLima, Fast, ultra-trace detection of Juvenile Hormone III from mosquitoes using mass spectrometry, Talanta, 2016:159:371-8

- P. Benigni, R. Marin, J.C. Molano-Arevalo, A. Garabedian, J.J. Wolff, M.E. Ridgeway, M.A. Park, F. Fernandez Lima, Analysis of protein complexes by Trapped Ion Mobility Spectrometry. Int. J. Ion Mob. Spectrom, 19.2-3 (2016): 95-104

- P. Benigni, J.D. DeBord, C.J. Thompson, P. Gardinali, F. Fernandez-Lima, Increasing Polyaromatic Hydrocarbon (PAH) Molecular Coverage during Fossil Oil 
Analysis by Combining Gas Chromatography and Atmospheric-Pressure Laser Ionization Fourier Transform Ion Cyclotron Resonance Mass Spectrometry (FT-ICR MS), Energy Fuels, 2016, 30 (1), 196-203

- $\quad$ P. Benigni, R. Marin, F. Fernandez-Lima, Towards unsupervised polyaromatic hydrocarbons structural assignment from SA-TIMS -FTMS data, Int J Ion Mobil Spectrom, 2015;18(3-4):151-7.

- P. Benigni, C.J. Thompson; M.E. Ridgeway, M.A. Park, and F. Fernandez-Lima, Targeted high resolution ion mobility separation coupled to ultra-high resolution mass spectrometry of endocrine disruptors in complex mixture, Anal Chem. 2015, 87 (8), 43215.

- Castellanos, P. Benigni, D.R. Hernandez, J. D. DeBord,; M. E. Ridgeway, M. A. Park, and F. Fernandez-Lima, Fast Screening of Polycyclic Aromatic Hydrocarbons using Trapped Ion Mobility Spectrometry - Mass Spectrometry, Anal Methods 2014, 6, 93289332.

\section{SELECTED PRESENTED PAPERS}

- $\quad$ P. Benigni, M. E. Ridgeway, M. A. Park, F. Fernandez-Lima Understanding the structural space of hydrocarbons in crude oil using TIMS-FT-ICR MS, 65th ASMS Conference on Mass Spectrometry and Allied Topics, Indianapolis, IN, June 4-8, 2017

- P. Benigni, F. Fernandez-Lima, Structural characterization of molecules in complex samples using TIMS coupled to FT-ICR MS. Asilomar Conference, Asilomar Conference Center, Pacific Grove, CA, October 14-17, 2016

- P. Benigni, F. Fernandez-Lima, Optimizing analytical tools for the structural characterization of molecules in complex samples by using High resolution IMS and FTICR MS, $21^{\text {st }}$ International Mass Spectrometry Conference, Toronto, Canada, August 21 26,2016

- P. Benigni, R. Marin, K. Sandoval, C.J. Thompson, M. E. Ridgeway, M. A. Park, P. Gardinali, F. Fernandez-Lima, Understanding tridimensional structural changes of hydrocarbon during oxygenation in the ocean using SA-TIMS-FT-ICR MS, $12^{\text {th }}$ European Fourier Transform Mass Spectrometry conference, April 5-8

- $\quad$ P. Benigni; K. Sandoval; C. Thompson; M. E. Ridgeway; M. A. Park; P. Gardinali; F. Fernandez Lima, "Monitoring the photo transformation of crude oils using SAIMS-FTICR MS”. 63 ${ }^{\text {rd }}$ ASMS Annual conferences, St. Louis MO, May 31- June 42015

- P. Benigni, C. J. Thompson, M. E. Ridgeway, M. A. Park, and F. Fernandez Lima, "Targeted high resolution Ion Mobility Separation couples to ultra high resolution mass spectrometry of endocrine disruptors in complex mixtures" $10^{\text {th }}$ North American FTMS Conference Key West Florida April 12-15 2015 